\title{
Detecção de objetos em vídeos usando misturas de modelos baseados em partes deformáveis obtidas de um conjunto de imagens
}

Leissi Margarita Castañeda León

\author{
DISSERTAÇÃO APRESENTADA \\ $\mathrm{AO}$ \\ Instituto De MatemáticA E EstatísticA \\ DA \\ Universidade DE SÃo PAUlo \\ PARA \\ OBTENÇÃO DO TÍTULO \\ $\mathrm{DE}$ \\ Mestre em CiÊnCIAS \\ Programa: Ciência da Computação \\ Orientador: Prof. Dr. Roberto Hirata Junior.
}

Durante o desenvolvimento deste trabalho o autor recebeu auxílio financeiro da FAPESP

São Paulo, 23 de outubro de 2012 


\title{
Detecção de objetos em vídeos usando misturas de modelos baseados em partes deformáveis obtidas de um conjunto de imagens
}

\author{
Esta dissertação contém as correções e alterações \\ sugeridas pela Comissão Julgadora durante a defesa \\ realizada por Leissi Margarita Castañeda León em 23/10/2012. \\ O original encontra-se disponível no Instituto de \\ Matemática e Estatística da Universidade de São Paulo.
}

Comissão Julgadora:

- Prof. Dr. Roberto Hirata Junior. (orientador) - IME-USP

- Prof. Dr. Carlos Hitoshi Morimoto - IME-USP

- Prof. Dr. Eduardo Alves do Valle Junior - UNICAMP 
Para meu filho Christopher e minha mãe. 
Agradeço e dedico inteiramente a dissertação, para quem é meu motivo de luta e perseverança, para meu adorado filho Christopher. Por ter vindo e por me fazer sorrir com pequenas coisas e sobretudo infinitamente feliz no dia-a-dia.

Vou dedicar este parágrafo em espanhol para minha família peruana. Para aquellos que amo mucho y que siempre están en mis pensamientos a pesar de la distancia. Para mi mamita adorada Ilsa, quien siempre da su mayor esfuerzo vida por verme feliz y realizada. A mi papá José, quien siempre me apoya en mis decisiones. Para mi hermano Roy, a quien debo mucho el estar aqui. Por brindarme siempre su apoyo tanto en la vida profesional como en lo personal; y por ser un ejemplo de lucha e superación. A mi hermanito $\boldsymbol{E d w i n}$, quien siempre fue mi mano derecha. Y para mi tía Gloria, a quien considero mi segunda mamá por todo lo que ha hecho por mí.

Agradeço ao meu esposo Jorge, por ser um grande apoio em todo momento, por ser o melhor pai e um bom companheiro. Pelos seus conselhos e amor nos anos juntos.

Para o meu orientador Roberto Hirata, quem é como um pai e grande amigo aqui no Brasil; agradeço infinitamente a confiança dele, os conselhos, correções, ideias e tudo. Acho que nunca acabaria de descrever o gratidão que sinto para com ele.

Agradeço àquelas pessoas especiais na minha vida. Para a minha amiga e "mãe" aqui no brasil: Jihan, por ter me ensinado a ser mãe e amiga, por ser uma maravilha de pessoa e por sempre me apoiar em tudo. Para os meus amigos: Talita, Leandro, David, Jesus e Rosario pela amizade, ajuda nas correções do texto, por estarem sempre presentes naqueles momentos que eu precisava de ajuda e pela convivência nestes anos na USP.

Aos membros da banca tanto da qualificação como da defesa.

E bom, esta dissertação nunca teria sido concebida sem o apoio financeiro da FAPESP, com o número de processo \#2010/04914 - 9, para qual me sinto muito grata. 


\section{Resumo}

LEÓN, L. M. C. Detecção de objetos em vídeos usando misturas de modelos baseados em partes deformáveis obtidas de um conjunto de imagens. $117 \mathrm{f}$. Dissertação - Instituto de Matemática e Estatística, Universidade de São Paulo, São Paulo, 2012.

A detecção de objetos, pertencentes a uma determinada classe, em vídeos é de uma atividade amplamente estudada devido às aplicações potenciais que ela implica. Por exemplo, para vídeos obtidos por uma câmera estacionária, temos aplicações como segurança ou vigilância do tráfego, e por uma câmera dinâmica, para assistência ao condutor, entre outros.

Na literatura, há diferentes métodos para tratar indistintamente cada um dos casos mencionados, e que consideram só imagens obtidas por um único tipo de câmera para treinar os detectores. Isto pode levar a uma baixa performance quando se aplica a técnica em vídeos de diferentes tipos de câmeras. O estado da arte na deteç̧ão de objetos de apenas uma classe, mostra uma tendência pelo uso de histogramas, treinamento supervisionado e, basicamente, seguem a seguinte estrutura: construção do modelo da classe de objeto, detecção de candidatos em uma imagem/quadro, e aplicação de uma medida sobre esses candidatos. Outra desvantagem observada é o uso de diferentes modelos para cada linha de visada de um objeto, gerando muitos modelos e, em alguns casos, um classificador para cada linha de visada.

Nesta dissertação, abordamos o problema de detecção de objetos, usando um modelo da classe do objeto criada com um conjunto de dados de imagens estáticas e posteriormente usamos o modelo para detectar objetos na seqüência de imagens (vídeos) que foram coletadas a partir de câmeras estacionárias e dinâmicas, ou seja, num cenário totalmente diferente do usado para o treinamento. A criação do modelo é feita em uma fase de aprendizagem off-line, utilizando o conjunto de imagens PASCAL 2007. O modelo baseia-se em uma mistura de modelos baseados em partes deformáveis (MDPM), originalmente proposto por Felzenszwalb et al. (2010b) no âmbito da detecção de objetos em imagens. Não limitamos o modelo para uma determinada linha de visada. Foi elaborado um conjunto de experimentos que exploram o melhor número de componentes da mistura e o número de partes do modelo. Além disso, foi realizado um estudo comparativo de MDPMs simétricas e assimétricas. Testamos esse método para detectar objetos como pessoas e carros em vídeos obtidos por câmera estacionária e dinâmica.

Nossos resultados não mostram apenas o bom desempenho da MDPM e melhores resultados que o estado da arte na detecção de objetos em vídeos obtidos por câmeras estacionárias ou di- 
nâmicas, mas também mostram o melhor número de componentes da mistura e as partes para o modelo criado. Finalmente, os resultados mostram algumas diferenças entre as MDPMs simétricas e assimétricas na detecção de objetos em diferentes vídeos.

Palavras-chave: deteç̧ão de objetos, mistura de modelos baseados em partes deformáveis (MDPM). 
LEÓN, L. M. C. Object detection in video using mixtures of deformable part models obtained from a image set. 117 f. M.Sc. text - Instituto de Matemática e Estatística, Universidade de São Paulo, São Paulo, 2012.

The problem of detecting objects that belong to a specific class of objects, in videos is a widely studied activity due to its potential applications. For example, for videos that have been taken from a stationary camera, we can mention applications such as security and traffic surveillance; when the video have been taken from a dynamic camera, a possible application is autonomous driving.

The literature, presents several different approaches to treat indiscriminately with each of the cases mentioned, and only consider images obtained from a stationary or dynamic camera to train the detectors. These approaches can lead to poor performaces when the tecniques are used in sequences of images from different types of camera. The state of the art in the detection of objects that belong to a specific class shows a tendency to the use of histograms, supervised training and basically follows the structure: object class model construction, detection of candidates in the image/frame, and application of a distance measure to those candidates. Another disadvantage is that some approaches use several models for each point of view of the car, generating a lot of models and, in some cases, one classifier for each point of view.

In this work, we approach the problem of object detection, using a model of the object class created with a dataset of static images and we use the model to detect objects in videos (sequence of images) that were collected from static and dynamic cameras, i.e., in a totally different setting than used for training. The creation of the model is done by an off-line learning phase, using an image database of cars in several points of view, PASCAL 2007. The model is based on a mixture of deformable part models (MDPM), originally proposed by Felzenszwalb et al. (2010b) for detection in static images. We do not limit the model for any specific viewpoint. A set of experiments was elaborated to explore the best number of components of the integration, as well as the number of parts of the model. In addition, we performed a comparative study of symmetric and asymmetric MDPMs. We evaluated the proposed method to detect people and cars in videos obtained by a static or a dynamic camera.

Our results not only show good performance of MDPM and better results than the state of the art approches in object detection on videos obtained from a stationary, or dynamic, camera, but also show the best number of components of the integration and parts or the created object. Finally, 
results show differences between symmetric and asymmetric MDPMs in the detection of objects in different videos.

Keywords: object detection, mixture of deformable part models (MDPM). 
Lista de Abreviaturas $\quad$ xi

Lista de Figuras $\quad$ xiii

Lista de Tabelas $\quad$ xvii

1 Introdução $\quad 1$

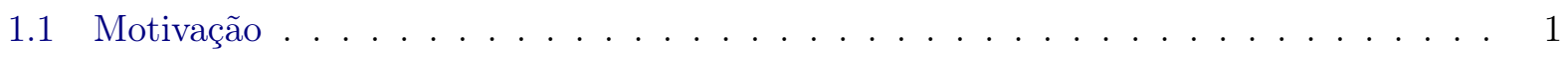

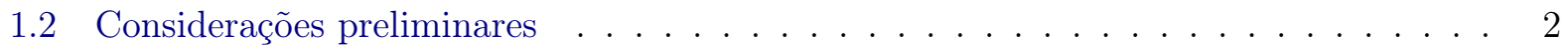

1.3 Desafios . . . . . . . . . . . . . . . . . . . . 4

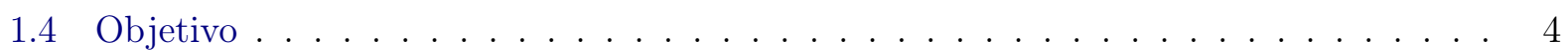

1.5 Estrutura da dissertação . . . . . . . . . . . . . . . . . . . 5

2 Fundamentos e revisão bibliográfica $\quad 7$

2.1 Definição do vocabulário . . . . . . . . . . . . . . . . . . . . 7

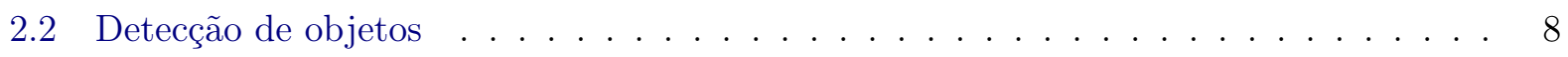

2.2 .1 Modelo de objetos . . . . . . . . . . . . . . . . . . 8

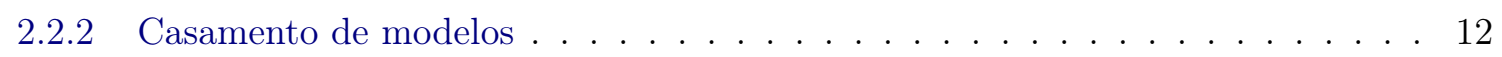

2.2 .3 Métodos de classificação . . . . . . . . . . . . . . . . . . . 13

2.2 .4 Revisão do estado do arte . . . . . . . . . . . . . . . . . . . . 14

3 Mistura de modelos baseados em partes deformáveis $\quad 17$

3.1 Extração de características . . . . . . . . . . . . . . . . . . . . . . 17

$3.2 \quad$ Filtro e Pirâmide de características . . . . . . . . . . . . . . . . . . . . . 19

3.3 Definição da MDPM . . . . . . . . . . . . . . . . . . . . . . . . . . 20

3.3 .1 Hipótese objeto . . . . . . . . . . . . . . . . . 21

3.4 Processo de casamento . . . . . . . . . . . . . . . . . . . . . 21

3.5 Indução do classificador . . . . . . . . . . . . . . . . . . . . . . 22

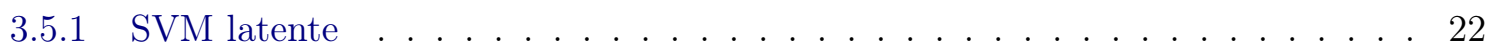

3.5 .2 SVM latente para as MDPMs . . . . . . . . . . . . 23

3.5.3 Treinamento das MDPMs simétricas . . . . . . . . . . . . . . . . 24

3.5.4 Treinamento das MDPMs assimétricas . . . . . . . . . . . . . 25 
4 Detecção de objetos em vídeos $\quad 29$

4.1 Criação de modelos . . . . . . . . . . . . . . . . . . . . . . . . . 29

4.1 .1 Conjunto de dados de imagens . . . . . . . . . . . . . . . . . . 30

4.2 Processo de deteç̧ão de objetos em vídeos . . . . . . . . . . . . . . . . . . . . 31

4.2.1 Vídeos para a classe carro com câmera estacionária . . . . . . . . . . . . . . 33

4.2.2 Vídeos para a classe carro com câmera dinâmica . . . . . . . . . . . . . 34

4.2 .3 Vídeos para a classe pessoa . . . . . . . . . . . . . . . . 35

$5 \quad$ Experimentos e resultados $\quad 39$

5.1 Métricas . . . . . . . . . . . . . . . . . . . . . . 39

5.2 Descrição dos experimentos . . . . . . . . . . . . . . . . . . . . 40

5.3 Parâmetros das MDPMs . . . . . . . . . . . . . . . . . . . . 41

5.4 Detecção de carros em vídeos obtidos por uma câmera estacionária . . . . . . . . . . . 46

5.4 .1 Conjunto de dados $1(\mathrm{E} 2) \ldots \ldots \ldots \ldots \ldots$

5.4 .2 Conjunto de dados $2(\mathrm{E} 3) \ldots \ldots \ldots \ldots \ldots$

5.5 Detecção de carros em vídeos obtidos por uma câmera dinâmica (E4) . . . . . . . . . 62

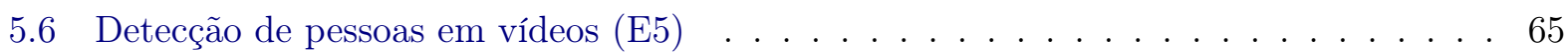

5.6 .1 Resultados . . . . . . . . . . . . . . . . . . 65

5.6.2 Unindo MDPMs simétricas e assimétricas . . . . . . . . . . . . . . . . . . 82

6 Conclusões $\quad 83$

6.1 Resumo das contribuições . . . . . . . . . . . . . . . . . . . 83

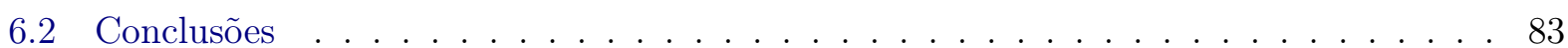

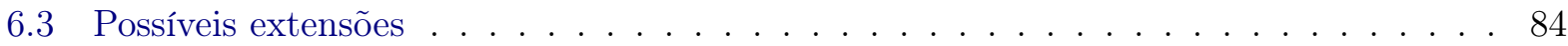

$\begin{array}{ll}\text { A Máquinas de vetores de suporte } & 87\end{array}$

A.1 SVM linear . . . . . . . . . . . . . . . . . . . . . . . 87

$\begin{array}{ll}\text { Referências Bibliográficas } & 89\end{array}$ 


\section{Lista de Abreviaturas}

HOG Histograma de gradientes orientadas (Histogram of Oriented Gradient)

MDPM Mistura de modelos baseados em partes deformáveis

(Mixture of Deformable Part Models)

SVM Máquinas de vetores de suporte (Support Vector Machine)

LSVM Máquinas de vetores de suporte latentes (Latent Support Vector Machine)

MI-SVM Multiple Instance-SVM

AP Média de precisão (average precision)

FPPI Falso positivo por imagem (False positives per image)

PASCAL VOC PASCAL Visual Object Classes Challenge 


\section{Lista de Figuras}

1.1 Tarefa de deteç̧ão. Imagens reproduzidas de Everingham et al. (2010). . . . . . . . . 1

1.2 Variedade de instâncias de quatro determinadas classes de objeto. Imagens reproduzidas de Everingham et al. (2010). . . . . . . . . . . . . . . . . . . 2

1.3 Detecção ao nível de instância. Imagem reproduzida de Sivic e Zisserman (2008) . . . 2

1.4 Detecção ao nível de classe. Imagem reproduzida de Felzenszwalb et al. (2008). . . . 3

1.5 Instâncias de objeto para a classe Carro. Reproduzidas de Everingham et al. (2010). 4

1.6 Organização dos capítulos do texto. . . . . . . . . . . . . . . . . 5

2.1 Imagem com um objeto identificado como avião (aeroplane), extraída de Everingham et al. (2010).

2.2 Diferencia entre o SIFT (esquerda) e o GLOH (direita). Reproduzida de Mikolajczyk e Schmid

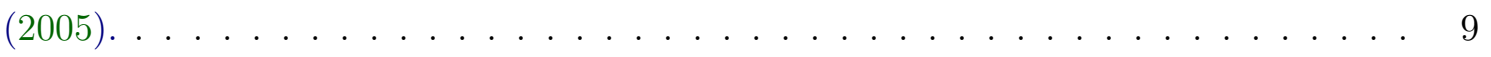

2.3 Descritor HOG. (a) Imagem original. (b) Imagem normalizada. (c) Imagem dos gradientes. (d) Criação dos descritores HOG por células. . . . . . . . . . . . . . . 10

2.4 Wavelets de Haar usadas para a detecção de faces. Reproduzida de Viola e Jones

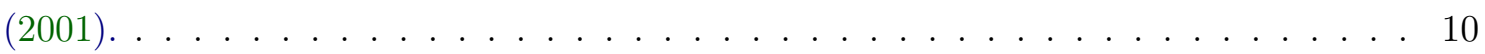

2.5 Modelo de partes deformável para o objeto pessoa. Reproduzida de Felzenszwalb e Huttenlocher

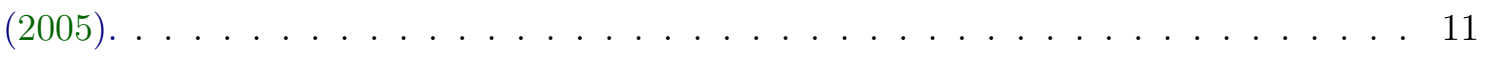

2.6 Representação do modelo de constelação (esquerda) e do modelo estrela (direita). . . 12

2.7 Modelo de estrutura pictórica. Imagem reproduzida de Fischler e Elschlager (1973). . 12

3.1 Uma representação de como fica o histograma de uma célula de C e D. . . . . . . . . 18

3.2 Representação do mapa de características HOG-31 dimensional. . . . . . . . . . . . . 19

3.3 Representação da pirâmide de características HOG-31 dimensional e a definição dos filtros nela. Imagem adaptada de Felzenszwalb et al. (2010b). . . . . . . . . . . . . . 20

3.4 Definição da MDPM. (a) Filtro raiz, (b) filtros parte de uma MDPM, (c) localização do filtro $F_{3}\left(v_{3}\right)$ e (d) exemplo de deformação das partes. . . . . . . . . . . 21

3.5 Exemplos positivos e negativos para a classe do objeto carro. . . . . . . . . . . . 24

3.6 Exemplo de bounding box latente. . . . . . . . . . . . . . . . . . 24

3.7 A MDPM simétrica de 3 componentes e 6 partes do modelo. . . . . . . . . . . . . . 26 
3.8 A MDPM assimétrica de 3 componentes e 6 partes do modelo. . . . . . . . . . . . . . 27

4.1 Exemplo de carros mais discriminativos de diferentes conjuntos de dados. Reproduzida de Torralba e Efros (2011) . . . . . . . . . . . . . . . . . . . . 30

4.2 Imagens de exemplo das duas classes selecionadas. Reproduzidas de Everingham et al.

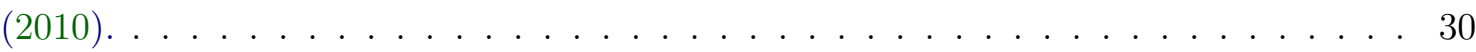

4.3 Processo de detecção num vídeo, usando a MDPM do objeto. . . . . . . . . . . . . . 31

4.4 Exemplo de tipos de câmeras. (a) Câmeras montadas num veículo. Reproduzida de Leibe et al. (2008b). (b) Área de trânsito de carros conhecida. . . . . . . . . . . . . 32

4.5 Imagens obtidas com uma câmera dinâmica. Extraídas de Leibe et al. (2007) . . . 32

4.6 Alguns quadros do vídeo $A V S S_{-} P V_{-} E V A L \ldots \ldots \ldots \ldots \ldots$

4.7 Alguns quadros do vídeo Leuven de Leibe et al. (2007) . . . . . . . . . . . . . . . . 35

4.8 Exemplos de dois diferentes vídeos de 4 categorias do PDds. . . . . . . . . . . . . . 37

5.1 MDPM assimétrica de 2 componentes e 8 partes para a classe pessoa. . . . . . . . . . 42

5.2 Curva precision vs recall para MDPMs simétricas (primeira coluna) e assimétricas (segunda coluna) de 2 e 3 componentes para a classe bicicleta (primeira fila), carro (segunda fila) e pessoa (última fila) . . . . . . . . . . . . . . . 44

5.3 algumas deteç̧ões em PASCAL VOC 2007, usando uma MDPM de 3 componentes

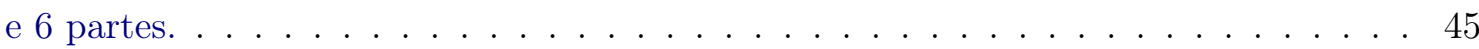

5.4 Deteç̧ões para o vídeo V1; usando uma MDPM (a) simétrica e (b) assimétrica. . . . 46

5.5 Deteç̧ões para o vídeo V2; usando uma MDPM (a) simétrica e (b) assimétrica. . . . 47

5.6 Deteç̧ões para o vídeo V3; usando uma MDPM (a) simétrica e (b) assimétrica. . . . 48

5.7 Detecções para o vídeo V4; usando uma MDPM (a) simétrica e (b) assimétrica. . . . 49

5.8 Detecções para o vídeo V5; usando uma MDPM (a) simétrica e (b) assimétrica. . . . 50

5.9 Deteç̧ões para o vídeo V6; usando uma MDPM (a) simétrica e (b) assimétrica. . . . 51

5.10 Deteç̧ões para o vídeo V7; usando uma MDPM (a) simétrica e (b) assimétrica. . . . 52

5.11 Deteç̧ões para o vídeo V8; usando uma MDPM (a) simétrica e (b) assimétrica. . . . 53

5.12 Deteç̧ões para o vídeo V9; usando uma MDPM (a) simétrica e (b) assimétrica. . . . 54

5.13 Curva precision vs. recall (PR) curva e curva miss rate vs. FPPI em $A V S S_{-} P V_{-} E V A L-$

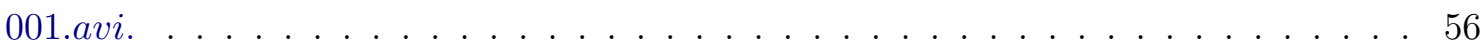

5.14 Resultado usando uma MDPM assimétrica de dois componentes e oito partes e o model2, em AVSS_PV_EVAL-001.avi. . . . . . . . . . . . . . 57

5.15 Curva precision vs. recall (PR) curva e curva miss rate vs. FPPI em $A V S S_{-} P V_{-} E V A L-$

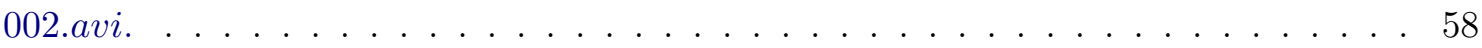

5.16 Curva precision vs. recall (PR) curva e curva miss rate vs. FPPI em $A V S S_{-} P V_{-} E V A L-$

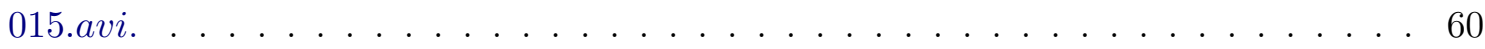

5.17 Curva precision vs. recall (PR) curva e curva miss rate vs. FPPI em $A V S S_{-} P V_{-} E V A L-$

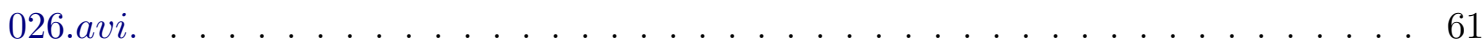

5.18 Curva precision vs. recall (PR) e curva miss rate vs. falso positivo por imagem (FPPI) no vídeo Leuven.

5.19 Resultados usando uma MDPM assimétrica de três componentes e oito partes e o model2 no vídeo Leuven. 
5.20 Curva de Precision vs. Recall e o AP para o vídeo V11, usando MDPMs simétricas e assimétricas de 3 componentes e 6,8 e 10 partes. . . . . . . . . . . . . 68

5.21 Curva de Precision vs. Recall e o AP para o vídeo V12, usando MDPMs simétricas e assimétricas de 3 componentes e 6,8 e 10 partes. . . . . . . . . . . . . . . . 69

5.22 Curva de Precision vs. Recall e o AP para o vídeo V14, usando MDPMs simétricas e assimétricas de 3 componentes e 6,8 e 10 partes. . . . . . . . . . . . . . 70

5.23 Curva de Precision vs. Recall e o AP para o vídeo V21, usando MDPMs simétricas e assimétricas de 3 componentes e 6,8 e 10 partes. . . . . . . . . . . . . 71

5.24 Curva de Precision vs. Recall e o AP para o vídeo V23, usando MDPMs simétricas e assimétricas de 3 componentes e 6,8 e 10 partes. . . . . . . . . . . . . . 72

5.25 Curva de Precision vs. Recall e o AP para o vídeo V26, usando MDPMs simétricas e assimétricas de 3 componentes e 6,8 e 10 partes. . . . . . . . . . . . . 73

5.26 Curva de Precision vs. Recall e o AP para o vídeo V31, usando MDPMs simétricas e assimétricas de 3 componentes e 6,8 e 10 partes. . . . . . . . . . . . . . . 74

5.27 Curva de Precision vs. Recall e o AP para o vídeo V32, usando MDPMs simétricas e assimétricas de 3 componentes e 6,8 e 10 partes. . . . . . . . . . . . . 75

5.28 Curva de Precision vs. Recall e o AP para o vídeo V43, usando MDPMs simétricas e assimétricas de 3 componentes e 6,8 e 10 partes. . . . . . . . . . . . 76

5.29 Curva de Precision vs. Recall e o AP para o vídeo V44, usando MDPMs simétricas e assimétricas de 3 componentes e 6,8 e 10 partes. . . . . . . . . . . . . . 77

5.30 Curva de Precision vs. Recall e o AP para o vídeo V45, usando MDPMs simétricas e assimétricas de 3 componentes e 6,8 e 10 partes. . . . . . . . . . . . 78

5.31 Curva de Precision vs. Recall e o AP para o vídeo V52, usando MDPMs simétricas e assimétricas de 3 componentes e 6,8 e 10 partes. . . . . . . . . . . . . . 79

5.32 Curva de Precision vs. Recall e o AP para o vídeo V53, usando MDPMs simétricas e assimétricas de 3 componentes e 6,8 e 10 partes. . . . . . . . . . . . . . . 80

5.33 Curva de Precision vs. Recall e o AP para o vídeo V54, usando MDPMs simétricas e assimétricas de 3 componentes e 6,8 e 10 partes. . . . . . . . . . . . . 81 


\section{Lista de Tabelas}

4.1 Resumo da quantidade de imagens e objetos etiquetadas como 'non-difficult', para duas classes, no conjunto de dados 2007 de Everingham et al. (a) . . . . . . . . . . 31

4.2 Sequencias de vídeo de transito de Chan e Vasconcelos (2005) utilizados nos experi-

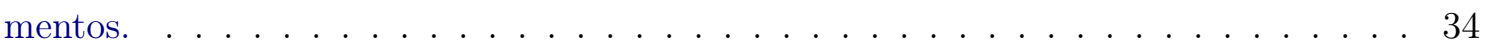

4.3 Categorias definidas nos vídeos do PDds. . . . . . . . . . . . . . . 36

4.4 Sequencias de vídeo do PDds usados nos experimentos. . . . . . . . . . . . . . . . 36

5.1 AP obtidos para a classe carro e pessoa, mudando os parâmetros das MDPMs simétricas e assimétricas. . . . . . . . . . . . . . . . . . . . . . . . 42

5.2 Comparação dos melhores AP obtidos usando o conjunto de dados de teste do PASCAL VOC $2007 . \ldots \ldots \ldots \ldots \ldots$

5.3 AP, recall, precision e FPPI usando MDPMs simétricas e assimétricas em $A V S S_{-} P V_{-} E V A L-$

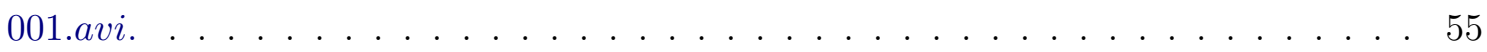

5.4 AP, recall, precision e FPPI usando MDPMs simétricas e assimétricas em $A V S S_{-} P V_{-} E V A L-$

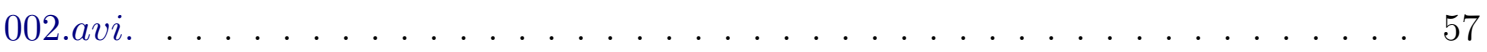

5.5 AP, recall, precision e FPPI usando MDPMs simétricas e assimétricas em $A V S S_{-} P V_{-} E V A L-$

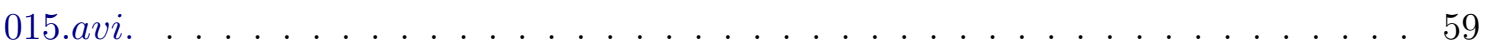

5.6 AP, recall, precision e FPPI usando MDPMs simétricas e assimétricas em $A V S S_{-} P V_{-} E V A L-$

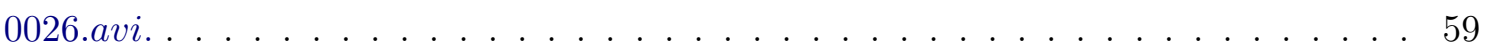

5.7 AP, precision, recall e FPPI usando MDPMs simétricas e assimétricas, e os modelos de Geiger et al. (2011), avaliados no vídeo Leuven. . . . . . . . . . . . . . . . . . 62

5.8 Precision, recall, AP e a pontuação F obtidos para a categoria 1, mudando os parâmetros das MDPMs simétricas e assimétricas. . . . . . . . . . . . . . . 65

5.9 Precision, recall, AP e a pontuação F obtidos para a categoria 2, mudando os parâmetros das MDPMs simétricas e assimétricas. . . . . . . . . . . . . 66

5.10 Precision, recall, AP e a pontuação F obtidos para a categoria 3, mudando os parâmetros das MDPMs simétricas e assimétricas. . . . . . . . . . . . . 66

5.11 Precision, recall, AP e a pontuação F obtidos para a categoria 4, mudando os parâmetros das MDPMs simétricas e assimétricas. . . . . . . . . . . . . . . 67 
5.12 Precision, recall, AP e a pontuação F obtidos para a categoria 5, mudando os parâmetros das MDPMs simétricas e assimétricas. . . . . . . . . . . . . . . 67

5.13 Precision, recall, AP e a pontuação F obtidos para alguns dos vídeos do PDds, mudando os parâmetros das MDPMs obtidos ao unir as MDPMs simétricas e assimétricas. 82 


\section{Introdução}

A tarefa de deteç̧ão ou localização de um objeto tenta responder à pergunta "onde está o objeto? (caso exista algum)"; ou seja, significa que conhecemos o objeto que estamos procurando na imagem/quadro (frame). A Figura 1.1 exemplifica mediante um bounding box a detecção ou localização do objeto, neste caso do avião.

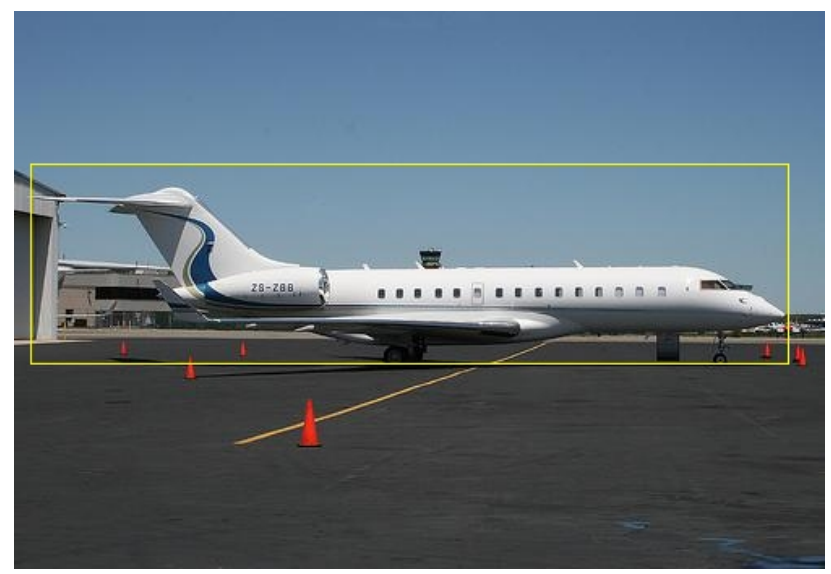

Figura 1.1: Tarefa de detecção. Imagens reproduzidas de Everingham et al. (2010).

A tarefa de deteç̧ão de objetos, vem sendo o processo chave quanto se trata com problemas de classificação ou segmentação. Por isso, o nosso trabalho foca-se na tarefa de detecção de objetos.

\subsection{Motivação}

Em imagens digitais, a tarefa de deteç̧ão de objetos é ainda um desafio e uma solução geral para qualquer classe de objeto, sob qualquer condição, está longe de ser conseguida. Para mitigar o problema, os investigadores criam métodos para detectar objetos sob certas restrições sobre os objetos, ou sobre o ambiente. Adicionalmente, para o caso de vídeos detectam ou em vídeos obtidos por uma câmera estacionária ou por uma câmera dinâmica de forma independente.

Um desafio comum quando se trata com classes de objetos é a grande variabilidade intraclasse, como é apresentada na Figura 1.2, que contém quatro classes diferentes de objetos e duas instâncias por classe. Embora os objetos da mesma classe compartilhem características semelhantes, eles apresentam diferenças devido a forma, cor, linha de visada entre outros. Diversos trabalhos tentam tratar com o reconhecimento deles em imagens e vídeos (Dalal, 2006; Dollár et al., 2012; Felzenszwalb et al., 2010b; García-Martín e Martínez, 2012; Szeliski, 2010; Wang et al., 2008).

Como possíveis aplicações, podemos frisar, às anotações/busca de objetos em coleções de vídeos, indexação semântica, vídeos de segurança, de assistência ao condutor, vigilância do tráfego, casamento de vídeos, entre outros. 


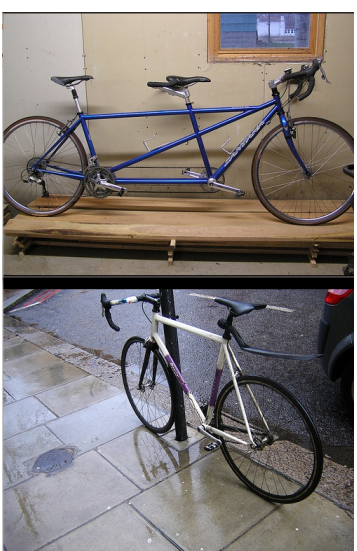

(a) Bicicleta

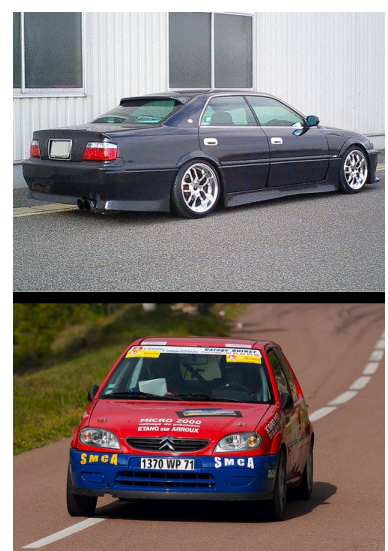

(b) Carro

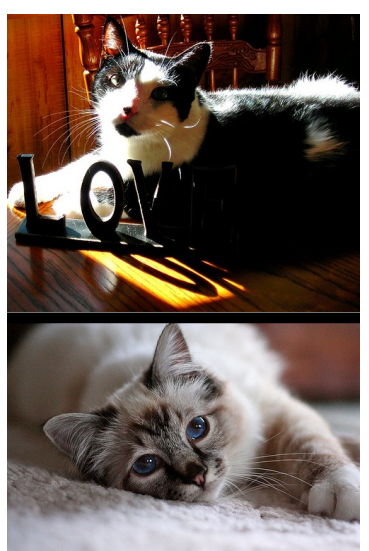

(c) Gato

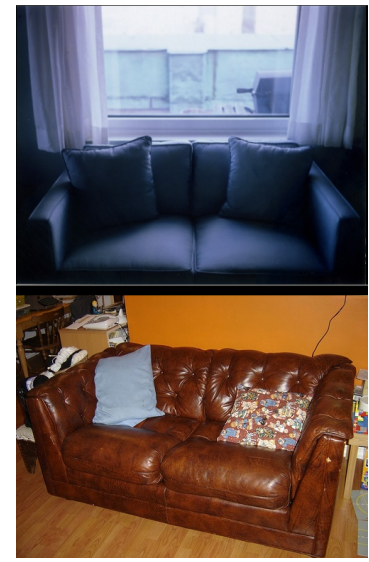

(d) Sofá

Figura 1.2: Variedade de instâncias de quatro determinadas classes de objeto. Imagens reproduzidas de Everingham et al. (2010).

\subsection{Considerações preliminares}

No estado-da-arte, além do tipo de vídeo utilizado e do tipo de objeto a ser detectado (sintético ou real), a tarefa de detecção de objetos em uma imagem ou vídeo pode ser vista em dois níveis diferentes: a detecção ao nível de instância de objeto e a detecção ao nível de classe do objeto.

- Na deteção ao nível de instância, o objeto pode estar representado por uma imagem como nos trabalhos de Sivic e Zisserman (2008) e Lampert (2009), ou por uma sequência de quadros do vídeo contendo um mesmo objeto, como o adotado por Rao (1997) e Bursuc et al. (2011). Através dos anos, diferentes algoritmos foram propostos, os quais focam na extração de linhas, contornos, ou superfícies em 3D a partir de imagens. Os trabalhos mais recentes extraem características que são invariantes à linha de visada em $2 \mathrm{D}$ e fazem casamento entre características.

Neste nível, deseja-se como resposta a detecção do mesmo objeto da consulta, isto é, um objeto em específico, em outra imagem/quadro onde ele pode estar com uma nova linha de visada, com oclusões parciais, etc. Por exemplo, na Figura 1.3, dada a imagem da bolsa, procura-se ela no vídeo e obtém-se como resposta um conjunto de deteç̧ões em diferentes cenas, onde observa-se o objeto com diferentes poses, oclusões e mudanças de iluminação.

\section{Objeto de consulta:}
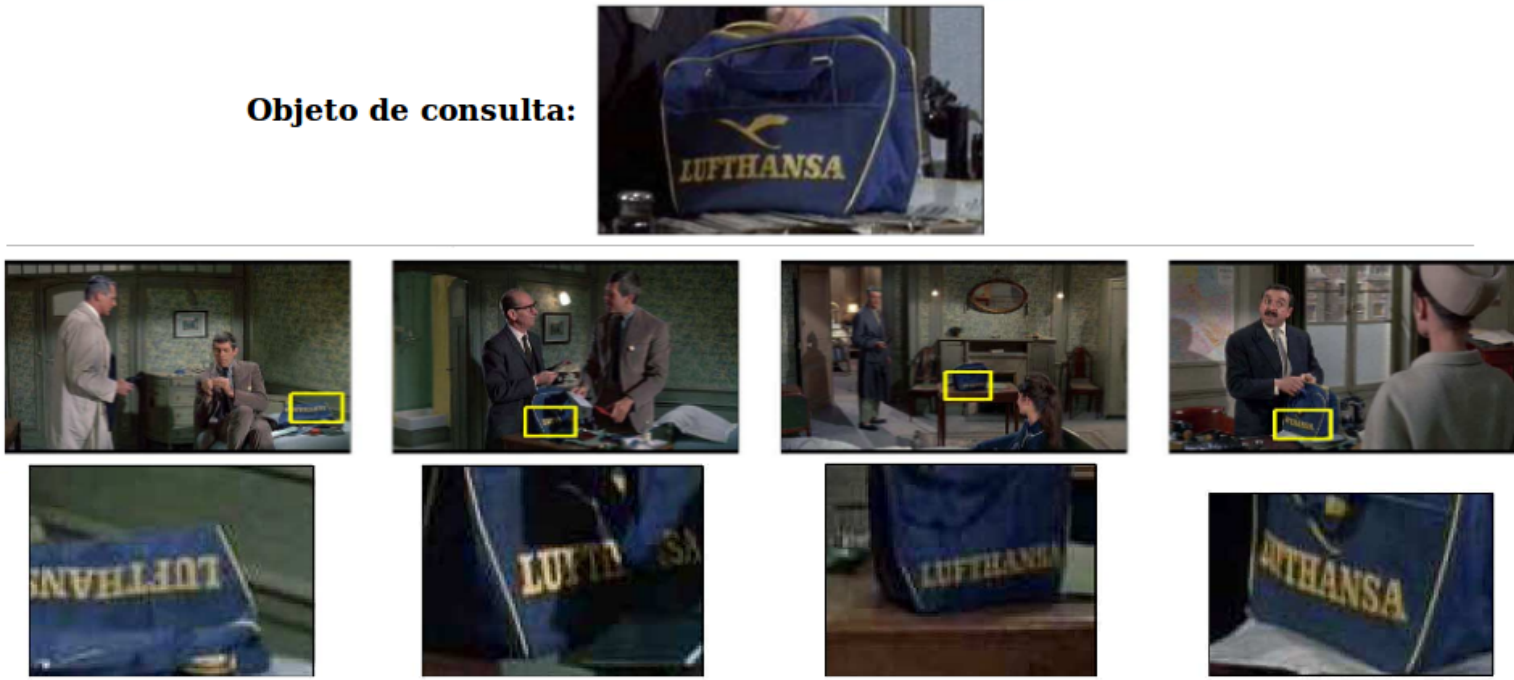

Figura 1.3: Deteção ao nível de instância. Imagem reproduzida de Sivic e Zisserman (2008) 
- Na detecção de objetos ao nível de classe, deseja-se detectar todos os objetos que pertencem a uma classe, por exemplo, detectar os objetos da classe bicicleta num conjunto de imagens, sem considerar a diferença no cor, linha de visada ou tipo de bicicleta. A literatura reporta que a classe do objeto pode estar representada: ou por um modelo construído à priori a partir de diferentes instâncias de uma mesma classe (Constantine e Tomaso, 2000; Dalal, 2006; Dalal et al., 2006; Felzenszwalb et al., 2008, 2010b; Fergus et al., 2003; Leibe et al., 2008a; Zheng e Liang, 2009); ou considerando a informação dos vídeos como, por exemplo, subtração ou extração do fundo (background) ou do objeto de interesse (foreground) como em Cheung e Kamath (2004), Magee (2004), Kamijo et al. (2001); estimação de movimento (Kamijo et al., 2001); entre outros. Como exemplo daqueles que consideram a informação de vídeo, temos os relacionados a vídeos de vigilância, onde existem os baseados em modelos estatísticos do fundo (McKenna et al., 1999; Wren et al., 1997), combinação de diferenças entre quadros e modelos estatísticos do fundo (Collins et al., 1999), etc.

O estado da arte na detecção de objetos de uma classe mostra uma tendência pelo uso de histogramas, treinamento supervisionado e basicamente seguem a seguinte estrutura: construção do modelo da classe de objeto, deteç̧ão de candidatos em uma imagem/quadro, e aplicação de uma medida sob esses candidatos.

Adicionalmente existem trabalhos que usam informação 2D ou 3D (Cornelis et al., 2008; Leibe et al., 2006, 2008b; Pepik et al., 2012; Sudowe e Leibe, 2011). No entanto, percebemos que a maioria dos trabalhos recentes consideram o uso de técnicas que combinam descritores de características locais com aprendizado de máquinas (Constantine e Tomaso, 2000; Felzenszwalb et al., 2010b; Harzallah et al., 2008; Sivic e Zisserman, 2008). Na Figura 1.4 mostram-se as detecções obtidas ao aplicar, em cada imagem, um modelo baseado em partes deformáveis (Felzenszwalb et al., 2008) criado para a classe bicicleta.
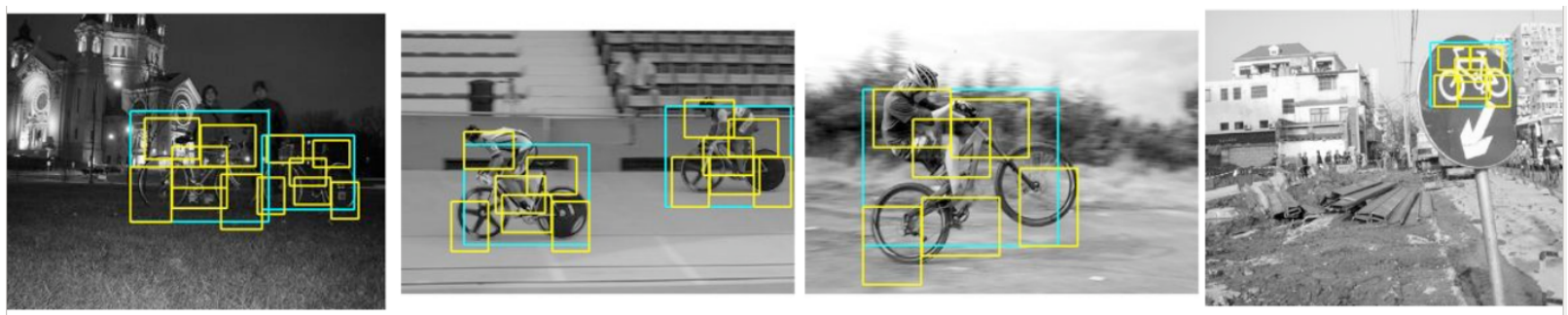

Figura 1.4: Deteç̧ão ao nivel de classe. Imagem reproduzida de Felzenszwalb et al. (2008).

O nosso interesse foca-se na detecção de objetos de uma mesma classe em vídeos. Quando tratase de vídeos, pode ser importante considerar a informação de fluxo óptico, descontinuidades de movimento, etc. Dalal (2006) introduz um esquema para detecção de pessoas em vídeos baseados em histogramas de orientações de diferenças locais, ou diferenças do fluxo óptico obtidos de duas imagens (quadros) consecutivas. No entanto, apesar de gerar um vetor contendo mais informações, não há melhora notável na performance, i.e. na tarefa de deteç̧ão, em relação à obtida considerando só o HOG definido em Dalal e Triggs (2005).

Além disso, na literatura, há diferentes métodos para tratar, ou com vídeos obtidos por uma câmera estacionária, ou por uma dinâmica, indistintamente, o que representa uma desvantagem. Isto pode levar a uma baixa performance quando aplica-se o processo em vídeos de diferentes tipos de câmeras que a usada no treinamento. e.g., treinando com dados de uma câmera dinâmica e testando em vídeos de uma câmera estacionária. Outra desvantagem é que usam-se diferentes modelos para cada linha de visada de um objeto, gerando muitos modelos e, em alguns casos, um classificador para cada linha de visada.

Neste sentido, o nosso interesse é a detecção de objetos de uma mesma classe em vídeos utilizando-se modelos de classe geradas com um conjunto de dados de imagens das quais só temos a informação da localização dos objetos. Para isso precisa-se enfrentar alguns desafios como é apresentado na próxima seção. 


\subsection{Desafios}

Os principais desafios a serem enfrentados são:

(a) Definir um modelo para uma classe a partir de imagens de exemplo das quais só temos a localização dos objetos nelas.

(b) A grande variabilidade intraclasse devido a características como cor, forma, etc. Por exemplo, de acordo com a localização da câmera, o visual de um mesmo objeto pode variar conforme a linha de visão, e podem existir oclusões. Veja a Figura 1.2 e com maior detalhe a Figura 1.5.
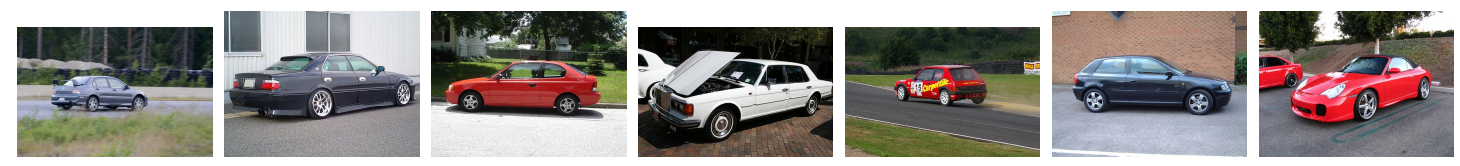

Figura 1.5: Instâncias de objeto para a classe Carro. Reproduzidas de Everingham et al. (2010).

(c) Tratar com vídeos sem um particular domínio restrito e cenas complexas, isto é, tratar com objetos estáticos, com objetos em movimento, com câmera estática ou câmera dinâmica.

(d) Criar um modelo a partir de um conjunto imagens e aplicá-lo sob um conjunto de vídeos.

Para o desafio (a) e (c), vamos explorar como funcionam as misturas de modelos baseados em partes deformáveis (MDPMs), geradas com um conjunto dados de imagens, na detecção de objetos de uma mesma classe em diferentes tipos de vídeo. Com respeito ao (d), segundo Torralba e Efros (2011), para o aprendizado de máquina, os conjuntos de dados são o mundo; mas em reconhecimento de objetos, o conjunto de dados é simplesmente uma representação do mundo. Então, qual que seria o melhor conjunto de imagens, dos disponíveis na literatura, para criar os modelos, tal que possam representar da melhor forma possível o mundo real e ao mesmo tempo ser aplicados em um outro conjunto de dados, neste caso em vídeos que representam também o mundo real?

\subsection{Objetivo}

O objetivo geral do trabalho de mestrado é a detecção de objetos de uma mesma classe em vídeos utilizando modelos de classe gerado com um conjunto de dados de imagens das quais só temos informação da localização dos objetos nelas. Para isso vamos explorar como funcionam as misturas de modelos baseados em partes deformáveis (MDPMs), geradas com um conjunto dados de imagens, na deteç̧ão de objetos de uma mesma classe em diferentes tipos de vídeo.

Desta forma definido o nosso objetivo e baseados nos diferentes desafios a serem enfrentados, propõem-se os seguintes objetivos específicos:

- Selecionar um conjunto de dados de imagens para criar as MDPMs, tal que o conjunto de dados tenha alta variabilidade intraclasse e possa representar o mundo real.

- Criar MDPMs de diferentes números de componentes e diferentes número de partes dos modelos de cada componente e analisar elas.

- Aplicar um detector de objetos, usando as MDPMS criadas, em diferentes tipos de vídeo, ou seja, serão definidos sem um domínio restrito: vídeos obtidos considerando uma câmera estática ou dinâmica e, também, onde os objetos podem estar estáticos ou em movimento. 


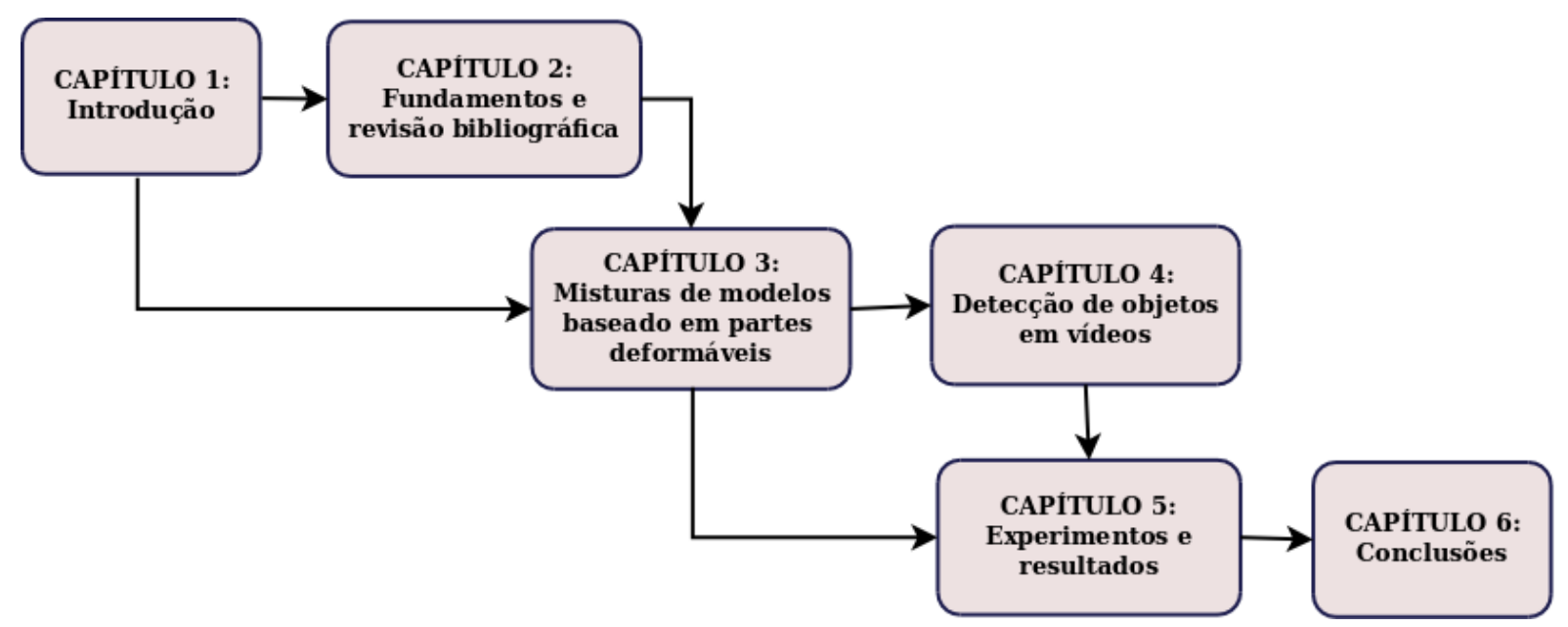

Figura 1.6: Organização dos capítulos do texto.

\subsection{Estrutura da dissertação}

Este capítulo apresentou uma visão geral do problema de reconhecimento e em especial a tarefa de detecção de objetos, assim como o objetivo da dissertação. O resto do texto está organizado seguindo a Figura 1.6.

- No Capítulo 2, começamos definindo o vocabulário usado a longo do texto. Logo, apresentamos uma revisão bibliográfica e o estado da arte com respeito à detecção de objetos que pertencem a uma determinada classe, tanto em imagens como em vídeos e as respectivas tarefas que ela envolve. Tarefas como a definição do modelo dos objetos, o processo de casamento de tais modelos e os métodos de classificação.

- O Capítulo 3, descreve o método para a construção das misturas de modelos baseados em partes deformáveis (MDPMs) simétricas e assimétricas respectivamente, de uma classe de objeto.

- O Capítulo 4, apresenta a metodologia proposta para a solução do problema de deteç̧ão de objetos em vídeos. Esse capítulo também descreve os conjuntos de dados utilizados no trabalho.

- Foram realizados diversos experimentos para determinar o números de componentes da mistura e o número de partes do modelo e para a tarefa de detecção de objetos em vídeos mesmo. Eles são apresentados no Capítulo 5 junto com as métricas usadas para avaliar os experimentos.

- Finalmente, no Capítulo 6, discutimos algumas conclusões e contribuições obtidas e descrevemos possíveis trabalhos futuros. 


\section{Fundamentos e revisão bibliográfica}

Neste capítulo, começamos definindo o vocabulário usado a longo do texto (Seção 2.1). Logo, apresentamos uma revisão bibliográfica e o estado da arte com respeito à detecção de objetos que pertencem a uma determinada classe, tanto em imagens como em vídeos (Seção 2.2). Mas como o processo de deteç̧ão implica em particular, tarefas como a definição do modelo dos objetos, o processo de casamento de tais modelos e os métodos de classificação então começamos fazendo uma revisão da bibliografia e o estado de arte delas, nas Subseções 2.2.1, 2.2.2 e 2.2.3 respectivamente.

\subsection{Definição do vocabulário}

Esta seção descreve a terminologia usada em toda a dissertação. A definição da terminologia é importante pelo fato de haver várias definições referentes a um mesmo conceito na literatura.

- Uma imagem (discreta) $I \in R^{M \times N}$, pode ser vista como um arranjo de duas dimensões de vetores, tipicamente em $[0,255]^{3}$, chamados pixels. Um pixel representa a intensidade (cor) do vetor $(i, j) \in M \times N$, onde $M, N \subset \mathbb{N}$.

- Um vídeo pode ser definido como o conjunto totalmente ordenado:

$$
V=\left\{Q_{M \times N}^{1}, Q_{M \times N}^{2}, \ldots Q_{M \times N}^{t n}\right\}
$$

onde $t \in\{1, \ldots, t n\}$ e $Q_{M \times N}^{t}$, representa uma imagem discreta no tempo $t$ (um quadro).

- Definimos como bounding box a área definida pelo retângulo de coordenadas $\left(i_{\text {esq }}, j_{\text {sup }}\right)$, canto inferior, e $\left(i_{\text {dir },} j_{\text {inf }}\right)$, canto inferior da imagem $\left(1 \leq i_{\text {esq }}<i_{\text {dir }} \leq M\right.$ e $\left.1 \leq j_{\text {inf }}<j_{\text {sup }} \leq N\right)$. Cabe mencionar que ao longo do texto usaremos o termo em inglês bounding box em vez de caixa delimitadora que bem poderia ser considerada como sua tradução.

- Chamaremos de objeto à área dentro da imagem definida por um bounding box. Por exemplo, o objeto na Figura 2.1 definido mediante bounding box (retângulo amarelo) define uma aeronave.

- Pode-se usar as características de um objeto ou imagem para descrever seu conteúdo, tais como forma, textura, cor, localização, etc. Essa descrição quantificada pode ser representada por pontos (vetores) em $\mathbb{R}^{n}$, aos quais chamaremos de descritores de características. Ao espaço $n$-dimensional, onde todos os $n$ elementos do descritor podem ser localizados, chamaremos de espaço de características.

- Chamaremos de deteç̧ão, ao problema de classificação binária, em uma determinada localização na imagem, definida pela função $D_{I}: \mathbf{x} \rightarrow\{-1,1\}$, com $\mathbf{x} \in \mathbb{R}^{n}$ e onde -1 representa se 


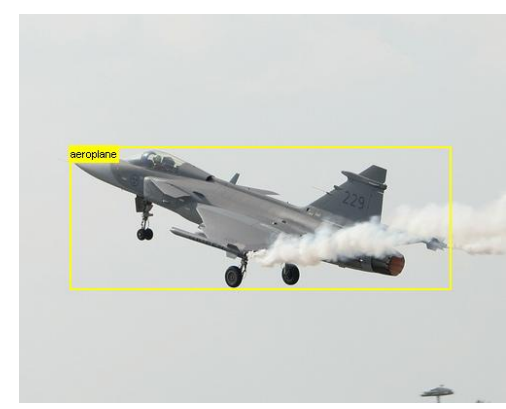

Figura 2.1: Imagem com um objeto identificado como avião (aeroplane), extraída de Everingham et al. (2010).

o descritor de caraterísticas definido por x não é casada na imagem $I$, e 1 em caso contrário. Informalmente faremos uso dos termos não detectou e detectou como referência aos valores -1 e 1 respectivamente.

- No contexto da deteç̧ão de objetos, a deformação de objetos é definida como uma região pertencente ao objeto, com localização não fixa, ou seja, que possui deslocamentos em uma determinada vizinhança (Hussein et al., 2009).

\subsection{Detecção de objetos}

Um detector de objetos pode ser visto como uma combinação de um conjunto de características da imagem/quadro e um algoritmo de detecção (Dalal, 2006).

\subsubsection{Modelo de objetos}

Diversas abordagens para modelagem de objetos tem sido propostas durante os últimos anos. As primeiras abordagens surgidas nos anos 70 e 80 definiam os modelos usando representações genéricas como coleções de características, modelos de configurações geométricas rígidas (i.e., cilindros) ou, no extremo oposto deste último, como modelos não estruturados. No entanto, alguns princípios muito importantes surgiram na década de 70, muitos dos quais são usados até hoje. Entre alguns desses princípios temos: a importância da forma na definição das classes de objetos, a importância da invariância com relação à linha de visada e a necessidade de representações hierárquicas. Esse último tornou-se menos comuns nos anos 80 .

No início dos anos 90, demonstrou-se interesse pela aparência dos objetos e por sistemas que pudessem detectar objetos complexos, ou seja, que sofressem oclusão, mudanças de iluminação, translação, rotação e escala. A partir do ano 2000, iniciou-se um aumento de interesse por modelos em que os objetos são descritos em termos de configurações espaciais de características locais (Amit, 2002; Fei-Fei et al., 2003), em representações baseadas em partes que sejam invariantes à translação, escala, rotação, iluminação, articulação e pontos de visada. No entanto, devido à grande quantidade de variação intraclasse existente, além de deformações dos objetos, a geração de um modelo para uma classe de objeto é um desafio. Para maiores detalhes da evolução dos modelos para a categorização de objetos através dos anos, pode-se consultar o trabalho de Dickinson (2009).

Entre os principais métodos em uso atualmente temos: a decomposição hierárquica do contorno do elemento, como o trabalho de Felzenszwalb e Schwartz (2007), os modelos de padrão (template) deformável (Coughlan et al., 2000; Yuille et al., 1992), os modelos baseados em partes (Amit e Trouve, 2007; Crandall et al., 2005; Fergus et al., 2003; Leibe et al., 2008a) e os modelos de estrutura pictórica propostos por Fischler e Elschlager (1973) e Felzenszwalb e Huttenlocher (2005).

Assim, uma variedade de detectores e descritores de características podem ser usados para analisar, descrever e casar imagens. No entanto, percebemos que a maioria dos trabalhos recen- 
tes consideram o uso de técnicas que combinam descritores de características locais com aprendizado de máquinas (Constantine e Tomaso, 2000; Felzenszwalb et al., 2010b; Harzallah et al., 2008; Sivic e Zisserman, 2008).

De forma geral, os modelos de objetos podem ser definidos para representar a aparência ou a configuração espacial dos objetos. As subseções seguintes descrevem cada uma delas.

\section{Representação da aparência}

Quando o objetivo dos modelos é representar a aparência, usam-se descritores de características ou padrões de descritores. Um descritor de características de uma imagem pode ser obtido concatenando todos os pixels da imagem, calculando a distribuição de intensidades da imagem ou tomando o gradiente da imagem.

Os descritores baseados na distribuição de intensidade usam histogramas na sua representação, sendo o mais representativo deste grupo o spin image, inicialmente proposto por Johnson e Hebert (1999) para ser usado sob pontos de interesse em 3D e logo em imagens por Lazebnik et al. (2003). O descritor baseado na distribuição de gradientes mais conhecido é o SIFT (Scale Invariant Feature Transform) (Lowe, 2004, 1999).

O SIFT é um descritor de características local, baseado na aparência dos objetos, e tem a propriedade de que é invariante a escala e rotação da imagem. Além disso, é robusto a pequenas alterações de pontos de vista, a mudanças de iluminação e à presença de ruído. Para a formação do descritor, pontos de interesse candidatos são obtidos a partir da detecção de extremos no espaçoescala de diferenças de gaussianas (DoG).

Como resultado do processo anterior tem-se uma grande quantidade de pontos, dos quais os de baixo contraste e aqueles localizados nas bordas são removidos restando apenas os pontos de interesse. Logo, para cada ponto de interesse é atribuída uma orientação. A orientação pode ser determinada por meio do cálculo de um histograma de 36 intervalos a partir da orientação do gradiente em uma região de $16 \times 16$ pixels, com centro em cada ponto de interesse. Pesos referentes à magnitude e uma função gaussiana circular (com $\sigma^{\prime}$ ) são aplicados para que orientações mais distantes tenham menor peso. Em seguida, cada sub-região de $4 \times 4$ pixels é projetada num único histograma de 8 intervalos. Finalmente, a criação do descritor de características para cada ponto de interesse é obtida pela concatenação dos histogramas da região, obtendo desta forma um descritor 128-dimensional.

Posteriormente, variantes do SIFT foram apresentadas. Dentre das variantes mais conhecidos temos o GLOH (Gradient Location and Orientation Histogram), proposto por Mikolajczyk e Schmid (2005), o qual usa estrutura de intervalos log-polar, como na Figura 2.2, no lugar de regiões de 4 usado por Lowe (2004).

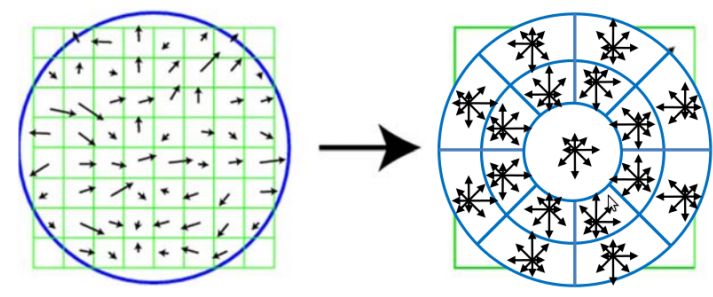

Figura 2.2: Diferencia entre o SIFT (esquerda) e o GLOH (direita). Reproduzida de Mikolajczyk e Schmid (2005).

Temos também, como variante do SIFT, o SURF (Speeded Up Robust Feature) (Bay et al., 2006), o PHOW (Pyramidal Histogram of Visual Words) em Lazebnik et al. (2006), o PCA-SIFT, proposto por Ke e Sukthankar (2004). No PCA-SIFT, inspirados no SIFT, eles calculam os gradientes em uma região de $39 \times 39$ pixels, gerando um vetor de 3042-dimensões; porém ao aplicar o PCA sob o vetor, obtém um novo vetor de 36-dimensões. Há também o SIFT FLOW em Liu et al. (2008), o BoF (Bag of Features) (Sivic et al., 2005), entre outros. 
Outro descritor, baseado na distribuição de gradientes, bastante usado é o HOG (Histogram of Oriented Gradients) proposto por Dalal e Triggs (2005). O HOG é um descritor de características robusto para mudanças de iluminação e cor da imagem. Representa a aparência e forma local do objeto. O descritor é definido sob uma grade densa e sobreposta de blocos com histogramas das orientações dos gradientes de uma imagem normalizados. Sua construção pode ser resumida nos passos apresentados na Figura 2.3, ou seja, aplica-se uma normalização global de cores na imagem, reduzindo assim os efeitos da iluminação.

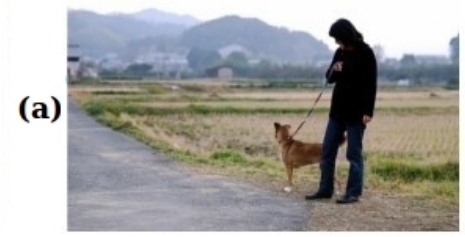

(b)
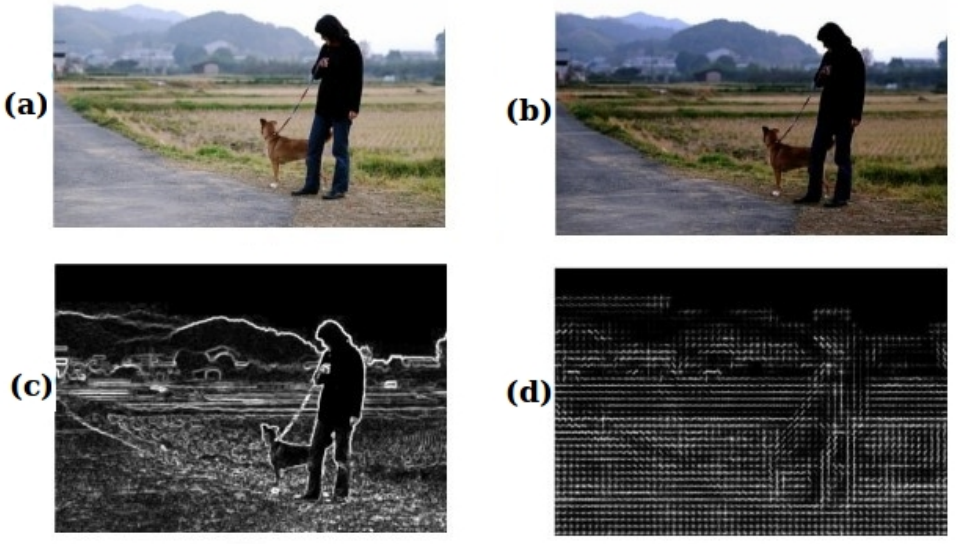

Figura 2.3: Descritor HOG. (a) Imagem original. (b) Imagem normalizada. (c) Imagem dos gradientes. (d) Criação dos descritores $H O G$ por células.

Para cada pixel da imagem calcula-se uma grade de gradientes (em imagens coloridas usa-se o canal da cor dominante). Logo, a imagem é dividida em regiões chamadas células (cells). Para cada célula, calcula-se um histograma de orientações de gradiente com um determinado número de intervalos, em que as magnitudes dos pontos da célula são usadas para acumular um voto no histograma de orientação. Uma normalização é feita em blocos, sendo cada bloco um grupo local de células. A normalização é feita devido às grandes variações de gradiente entre os pontos da imagem. Finalmente, aos histogramas normalizados chama-se "descritores HOG".

Há também adaptações do HOG, como o PHOG (Pyramidal Histogram of Oriented Gradients) (Bosch et al., 2007), o HOG avaliado em múltiplas escalas, como em Felzenszwalb et al. (2008), considerando características sensíveis e insensíveis ao contraste (Felzenszwalb et al., 2010b) e o Motion HOG descrito em Dalal (2006) e proposto para vídeos. Este último combina descritores estáticos (HOG convencional) com descritores obtidos por fluxo óptico.

As wavelets de Haar também são muito usadas para representar a aparência dos objetos. Entre alguns dos trabalhos que consideram esta abordagem para a detecção de objetos temos os trabalhos de Constantine e Tomaso (2000) e de Viola e Jones (2001). Na Figura 2.4 apresenta-se um exemplo de como são usadas as wavelets de Haar para extrair características para uma posterior detecção de faces, no qual a primeira característica mede as diferenças de intensidade entre os olhos e as bochechas, e a segunda entre os olhos e a ponta do nariz.

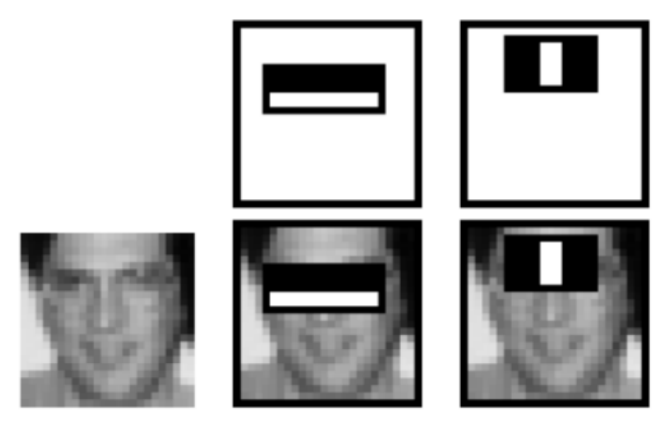

Figura 2.4: Wavelets de Haar usadas para a detecção de faces. Reproduzida de Viola e Jones (2001). 


\section{Representação da configuração espacial}

Quando se trata de representar a configuração espacial dos objetos de uma classe, os métodos referem-se basicamente ao uso de modelos baseado em partes (Amit e Trouve, 2007; Crandall et al., 2005; Crandall e Huttenlocher, 2006; Fergus et al., 2003; Leibe et al., 2008a; Zhu et al., 2010) ou modelo/padrão deformável (Cootes e Taylor, 2001; Coughlan et al., 2000; Yuille et al., 1992).

As abordagens baseadas em representação de componentes ou partes dos objetos são mais robustas às oclusões parciais. Do mesmo modo, os modelos de padrão deformável foram definidos para tratar o problema da variação intraclasse. Como consequência, os modelos de partes deformáveis (veja a Figura 2.5), constituem uma alternativa considerada no estado do arte (Felzenszwalb et al., 2008; Felzenszwalb et al., 2010b), pois conseguem capturar outros aspectos na aparência e detalhes dos objetos, tratando-os como uma versão deformável de um padrão.
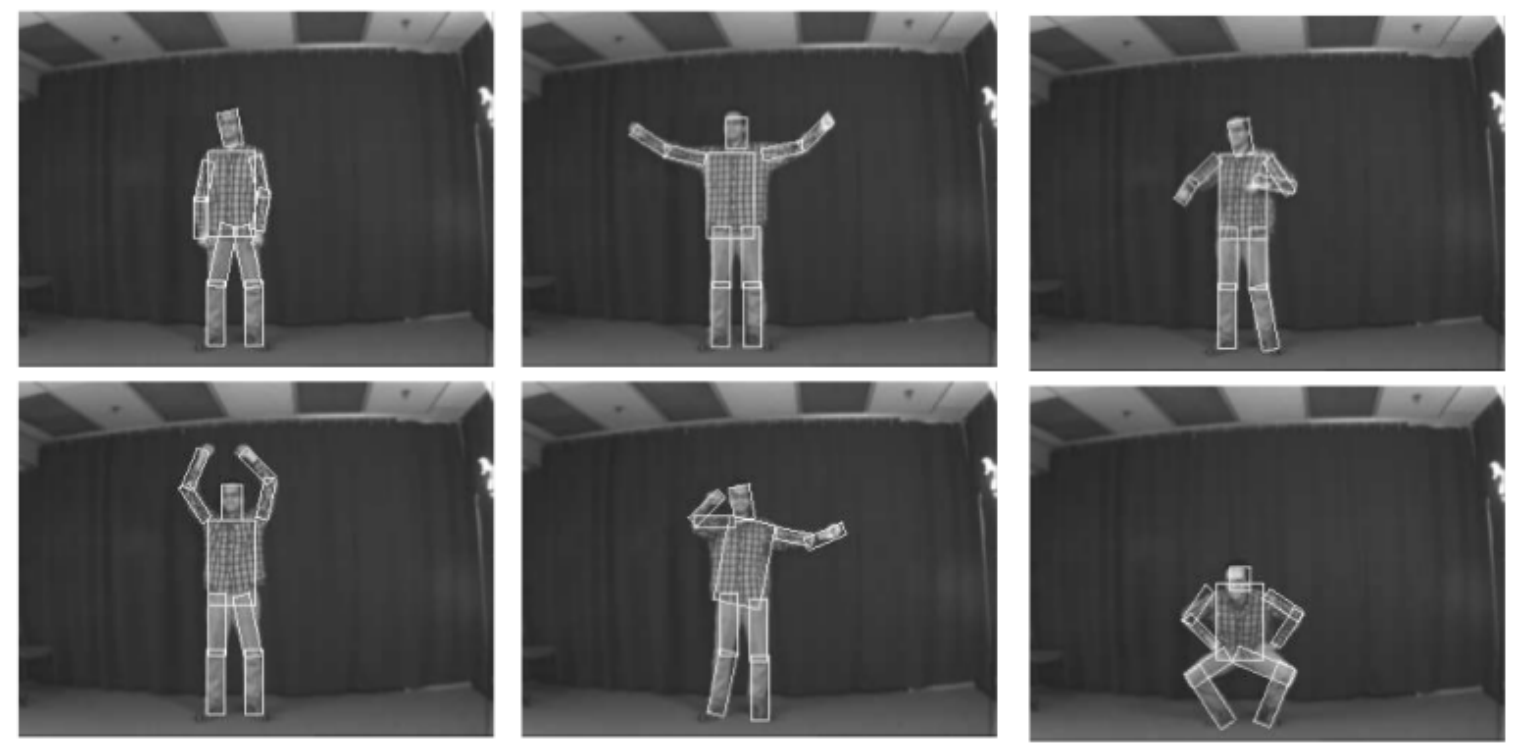

Figura 2.5: Modelo de partes deformável para o objeto pessoa. Reproduzida de Felzenszwalb e Huttenlocher (2005).

Muitas vezes, um modelo deformável 2D não consegue capturar muito bem as variações extremas de forma e aparência. Por outro lado, uma mistura de modelos (Felzenszwalb et al., 2010b) é uma alternativa simples para tratar com o problema. Desta forma, a ideia principal de uma mistura de modelos é decompor complexas categorias visuais dos objetos num conjunto de simples subcategorias, onde as subcategorias são automaticamente aprendidas e cada uma represente uma variação de aspecto e pose.

Dentre as definições dos modelos de partes deformáveis temos diferentes topologias para representar a conexão entre partes, tais como:

- Saco de palavras (bag of words), no qual não existe uma relação geométrica entre as diferentes partes ou características. Essa estratégia pode ser muito eficiente, mas não considera a relação espacial entre as partes o qual é um limitante.

- Modelo de constelação, em que as partes são tratadas como um conjunto de localizações determinado por um operador de ponto de interesse. A principal vantagem deste modelo é que ele trabalha bem para várias classes de objeto e a desvantagem é o custo computacional, devido ao fato de ser um modelo totalmente conectado. Fergus et al. (2003) considerou usar partes do objeto invariantes a escala, modelando-as por uma distribuição de probabilidade espacial conjunta e uma distribuição da aparência, resultando assim num modelo de constelação.

- Modelo estrela, quando existe relação direta entre a raiz do modelo e as partes. Trata-se de uma maneira simples de representar as deformações das partes de um objeto. Este modelo é 
usado, por exemplo, por Andriluka et al. (2008) e Felzenszwalb et al. (2008). Na Figura 2.6 é exibida, de maneira gráfica, a principal diferença entre os modelos de constelação e de estrela, que são as conexões entre as partes.
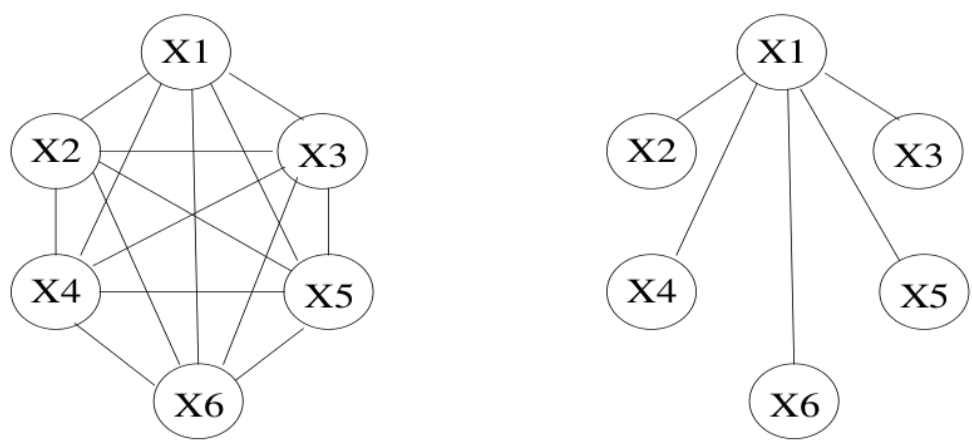

Figura 2.6: Representação do modelo de constelação (esquerda) e do modelo estrela (direita).

- Estrutura pictórica, é um modelo que permite uma eficiente inferência das partes de um objeto representado por uma constelação delas. A ideia principal da estrutura pictórica, como mostrado na Figura 2.7, apresentada inicialmente por Fischler e Elschlager (1973), é representar um objeto como um conjunto de componentes rígidas com certas restrições de disposição espacial entre pares de partes (custo) e algoritmos de casamento baseados em programação dinâmica. Já o trabalho de Felzenszwalb e Huttenlocher (2005), introduz um algoritmo de casamento em tempo linear e mostrou resultados em deteç̧ão de faces e em tarefas de estimação de pose de pessoas.

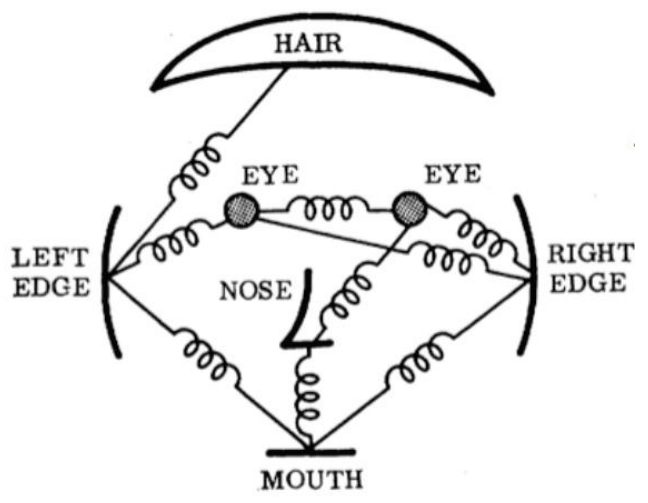

Figura 2.7: Modelo de estrutura pictórica. Imagem reproduzida de Fischler e Elschlager (1973).

\subsubsection{Casamento de modelos}

Dados os modelos de duas imagens/quadros, o passo seguinte é o casamento entre eles. O casamento de modelos foi a primeira abordagem usada para tratar o problema de detecção de objetos. O problema de casamento pode ser visto em duas componentes separadas: em uma estratégia de casamento (distância euclideana, vizinho mais próximo, etc.) e em uma estrutura de dados e algoritmos eficientes para calcular o casamento da forma mais eficiente possível (hashing, $k$ - $d$ trees, etc.).

Uma das técnicas mais usadas no casamento de modelos é a baseada em janela, também conhecida como janela de deteç̧ão deslizante (Bosch et al., 2007). Esta técnica funciona como um detector em que, ao calcular a pontuação de casar a janela do modelo ou imagem sob a sub-janela da imagem de busca, classifica-a usando um limiar. O processo é repetido em diferentes escalas da imagem. 
Considerando como janela deslizante o modelo do objeto, o casamento entre o modelo e uma imagem de uma cena pode ser feito pixel a pixel. Para o caso de descritores globais pode ser considerada alguma medida de distância entre os descritores e, para o caso de modelos deformáveis, adiciona-se um custo de deformação à medida de similaridade. O casamento de modelos deformáveis implica em um custo computacional maior.

A abordagem de um casamento pixel a pixel apresenta deficiências principalmente devido a alterações da linha de visada, oclusões e deformações de partes dos objetos (Nevatia e Binford, 1977). Para o caso de casamento de descritores, é definida uma estratégia de casamento e uma estrutura de dados e algoritmos tal que o casamento seja realizado de maneira rápida. Por exemplo, para obter o casamento dos descritores SIFT de duas imagens, Lowe (2004) propõe um algoritmo rápido de vizinhos mais próximos baseado na abordagem euclideana para medir a similaridade entre dois descritores. O algoritmo é uma modificação do algoritmo $k$-d tree (Bentley, 1975), chamado algoritmo do Best-bin-first (BBF) proposto por Beis e Lowe (1997). Para aumentar a robustez e eliminar falsos casamentos, são rejeitados os pontos de interesse para os quais a razão do vizinho mais próximo ao segundo mais próximo é maior que 0.8 .

A principal diferença entre os métodos que casam as estruturas de objetos calculando um custo de deformação é a forma como os pontos de referência são encontrados e a função de energia escolhida. A função de energia é usada para calcular o custo de deformação. Como exemplos podemos citar a distancia euclideana, thin plate splines (TPS), etc. Para o caso dos modelos de estrutura pictórica (veja a Figura 2.7), o modelo é representado por um grafo $G=(V, E)$, em que $V=$ $\left\{v_{1}, \ldots, v_{n}\right\}$ são as partes e $\left(v_{i}, v_{j}\right) \in E$ indica as conexões entre as partes. Na sequência, o casamento é realizado por meio de programação dinâmica e da minimização da função de energia, que possui a forma:

$$
E(L)=\sum_{i=1}^{n} m_{i}\left(l_{i}\right)+\sum_{\left(v_{i}, v_{j}\right) \in E} d_{i j}\left(l_{i}, l_{j}\right),
$$

em que $m_{i}\left(l_{i}\right)$ é o custo para localizar a parte $i$ na localização $l_{i}$ e $d_{i j}\left(l_{i}, l_{j}\right)$ é o custo de deformação.

\subsubsection{Métodos de classificação}

Por trás de um modelo de classe gerado na fase de treinamento, temos os métodos de classificação. Os métodos de classificação baseados em descritores de partes locais podem ser divididos em: (1) abordagens discriminativas, como as máquinas de vetores de suporte (SVMs, do inglês Support Vector Machines), boosting, redes neurais, entre outros; ou (2) generativas, como os bayesianos.

\section{Abordagens discriminativas}

Técnicas de aprendizagem de máquina como as SVMs (Vapnik, 1995) e boosting (Schapire, 2002) são populares como classificadores para reconhecimento de objetos, por causa de sua habilidade para selecionar automaticamente os descritores ou características relevantes a partir de um conjunto grande de características, por sua performance e sua facilidade de uso.

As SVMs propostas por Vapnik (1995), é um método usado para resolver problemas de classificação de padrões e problemas de regressão. Informalmente, pode-se dizer que a SVM procura o hiperplano que maximiza a margem entre a classe objeto e a classe não objeto sob um espaço de características ou uma versão kernelizada dele, dada como entrada. Existem diferentes variantes na literatura tais como: one class SVM (Schölkopf et al., 2001), C-SVM (Cortes e Vapnik, 1995; Vapnik, 1998), $\nu$ SVM (Schölkopf et al., 2000), SVM estrutural (Joachims et al., 2005; Tsochantaridis et al., 2004), SVM latente (Felzenszwalb et al., 2010b; Yu e Joachims, 2009), entre outros, cada um orientado para um problema em particular. Os algoritmo de implementação mais usados são o SMO (Platt, 1999) e o algoritmo "shrinking" (Joachims, 1999a). Por outro lado existem muitas bibliotecas, entre as mais populares temos o LIBSVM (Chang e Lin, 2011) e o SVMlight (Joachims, 1999b). Maior detalhe das SVMs lineares no Apêndice A. 
O AdaBoost, proposto em Schapire (2002), combina uma coleção de classificadores fracos (classificadores que cometem erros) para formar um melhor classificador. Ele é usado particularmente para construir cascatas tal que em cada nível da cascata sejam escolhidas as características, descritores, ou padrões mais relevantes e rejeitados os outros. Ele é rápido para a fase de teste, mas é conhecido por ser lento para treinar, precisando de muitos exemplos de treinamento.

\section{Abordagens generativas}

Nesta abordagem fazem uso da relação de verossimilhança para classificar. Para aprender, podem usar modelos bayesianos que usam o algoritmo EM (Expectation-Maximization) ou com modelos de probabilidades condicionais que incluem a posição e escala das características assim como sua aparência. Não entraremos em detalhes para este caso porque não é de nosso interesse neste texto.

\subsubsection{Revisão do estado do arte}

Dentre as técnicas criadas para a detecção de objetos, algumas delas tem como objetivo a velocidade/rapidez e a eficiência. Outras, entretanto, focam-se na acurácia, em enfrentar o desafio da linha de visada obtendo assim um detector mais genérico. Por exemplo, em Viola e Jones (2001), os autores concentraram-se em construir um sistema otimizado em tempo para a detecção de faces usando na fase de aprendizado uma cascata de classificadores simples baseados nas wavelets de Haar. Nos últimos anos, esta abordagem mostrou-se eficiente e dá bons resultados na acurácia de detecção de objetos. Entretanto, ela não modela explicitamente as variações locais numa estrutura do objeto, precisando de um número grande de exemplos de treinamento para conseguir aprender as mudanças permitidas em uma aparência global.

Uma das propriedades importantes num processo de detecção, ainda mais se for usado em situações do mundo real, é a robustez às mudanças de escala. Mesmo estando a câmera estacionária, os objetos de interesse podem estar presentes em diferentes escalas com respeito à câmera. Por isso, é importante que as diferentes abordagens considerem um certo grau de variação de escala. A maioria de métodos de detecção de objetos tratam o problema de variação de escala realizando uma busca exaustiva em todas as possíveis localizações e escalas do objeto (Constantine e Tomaso, 2000; Dalal e Triggs, 2005; Felzenszwalb et al., 2008). Assim, no estado da arte, a detecção de objetos é baseada na técnica de janela deslizante (multi-escala) ou variações dela, embora os principais problemas da técnica sejam as múltiplas respostas de detecção para um mesmo objeto e a relação de aspecto da janela.

Em relação aos objetos, temos que aqueles que maior atenção receberam na tarefa de detecção de objetos, além das faces, são as pessoas e os carros. A literatura em relação à detecção de pessoas e carros é extensa, e entre alguns deles temos Andriluka et al. (2008); Constantine e Tomaso (2000); Dalal e Triggs (2005); Felzenszwalb et al. (2008); Felzenszwalb e Huttenlocher (2005); Leibe et al. (2008a); Mikolajczyk e Schmid (2005); Papageorgiou (2000); Schneiderman e Kanade (2000); Sun et al. (2006); Torralba et al. (2008); Wang et al. (2008) e entre os trabalhos mais recentes estão Dollár et al. (2012); Felzenszwalb et al. (2010a,b); Feris et al. (2011); García-Martín et al. (2012); López-Sastre et al. (2011); Niknejad et al. (2011, 2012b); Schiele (2012).

Em 2008, houve o desafio de detecção do PASCAL VOC (Everingham et al., b), onde trabalhos no estado da arte na detecção de objetos foram submetidos. As abordagens, em sua maioria, fazem uso do SIFT ((Lowe, 2004, 1999)), histograma de características HOG ((Dalal e Triggs, 2005)) e aprendizagem SVM. Entre os trabalhos do desafio com melhores resultados, em média de precisão e curva precisão-revocação, estão os de Harzallah et al. (2008) e de Felzenszwalb et al. (2010b).

O trabalho de Harzallah et al. (2008), baseia-se na análise por janelas deslizantes. Fazem uma combinação contextual dos processos de detecção (localização) e classificação. Usam um classificador SVM linear para uma pre-seleção de possíveis localizações fazendo filtragem de janelas negativas e um SVM não linear para calcular a pontuação da hipótese do objeto. Primeiro aplicam o SVM linear para cada sub-janela da imagem usando descritores HOG. Depois, aplicam a função final de pontuação, que é dada pelo SVM não-linear com um kernel $\chi^{2}$ e descritores obtidos combinando 
HOG e SIFT, mas só nas regiões candidatas. Para o treinamento do classificador fornecem exemplos positivos e negativos para cada linha de visada do objeto definido (lado, frente, não especificado), desta forma o classificador encontra-se limitada para tratar só com objetos em aquelas linha de visada.

O trabalho de Felzenszwalb et al. (2010b) abordou o problema na análise por janelas deslizante usando misturas de modelos multi-escala de partes deformáveis para cada classe. Baseia-se nos trabalhos de Dalal e Triggs (2005); Felzenszwalb et al. (2008), mas considerando cada modelo como uma estrutura pictórica, ou seja, definido por filtros lineares (raiz e partes), por um conjunto de localizações permitidas para cada parte em relação à raiz e por um custo de deformação para cada localização da parte. Para a geração dos modelos é dado um conjunto de imagens etiquetadas com bounding boxes e SVM linear e latente. Reformularam assim o MI-SVM (Andrews et al., 2003) em termos de variáveis latentes ao qual chamaram de SVM latente (LSVM).

Diversas abordagens para modelagem de objetos tem sido propostas durante os últimos anos. Entre os principais métodos em uso atualmente temos: a decomposição hierárquica do contorno do elemento, como o trabalho de Felzenszwalb e Schwartz (2007), os modelos de padrão (template) deformável (Coughlan et al., 2000; Yuille et al., 1992), os modelos baseados em partes (Amit e Trouve, 2007; Crandall et al., 2005; Fergus et al., 2003; Leibe et al., 2008a) e os modelos de estrutura pictórica propostos por Fischler e Elschlager (1973) e Felzenszwalb e Huttenlocher (2005). Assim, uma variedade de detectores e descritores de características podem ser usados para analisar, descrever e casar imagens. De forma geral, os modelos de objetos podem ser definidos para representar a aparência ou a configuração espacial dos objetos. Percebemos que a maioria dos trabalhos recentes consideram o uso de técnicas que combinam descritores de características locais com aprendizado de máquinas (Constantine e Tomaso, 2000; Felzenszwalb et al., 2010b; Harzallah et al., 2008; Sivic e Zisserman, 2008).

A literatura reporta que modelos baseados na aparência, como os modelos deformáveis (Dalal, 2006; Felzenszwalb e Huttenlocher, 2005; Felzenszwalb et al., 2010b; Schneiderman e Kanade, 2000; Zheng e Liang, 2009), alcançam um bom desempenho em cenários difíceis (Feris et al., 2011). Variantes dos modelos deformáveis (Felzenszwalb et al., 2010b), foram propostos, ou considerando a deteç̧ão de carros na noite (Niknejad et al., 2011), ou modificando o tamanho das partes (Lv et al., 2012; Niknejad et al., 2012a), ou combinando com outras técnicas (Niknejad et al., 2012b), ou até considerando informação 3D (Schiele, 2012).

Em alguns casos os modelos dos objetos são criados considerando imagens de uma linha de visada em específico, o qual limita para outros pontos de visada, e o usar eles em cenários diferentes (Jain et al., 2011; Leibe et al., 2008b). Adicionalmente, existem detectores específicos para cada linha de visada (López-Sastre et al., 2011), o que implica tempo adicional no treinamento e processamento, além da necessidade de ter um conjunto de dados para treinar com imagens para cada linha de visada do objeto.

Outros trabalhos baseados em Felzenszwalb et al. (2008), ou seja, em modelar as partes dos objetos, consideram compartilhar informação das partes para a detecção de objetos de uma mesma classe (Fidler e Leonardis, 2007; Thomas et al., 2006; Torralba et al., 2008; Zhu et al., 2008, 2010). Em Thomas et al. (2006), usam-se duas camadas de estruturas, o que dificulta no caso de objetos com diferentes intervalos de escala em sua estrutura. Em Fidler e Leonardis (2007), usam-se estruturas hierárquicas e compartilhamento de partes sem considerar os diferentes pontos de visada. Em Torralba et al. (2008), compartilham-se características usando boosting. Em Zhu et al. (2010) considera-se representar um objeto em diferentes linhas de visada com uma família de Modelos de Composição Recursiva (RCMs), criando-se um dicionário. Cada RCMs representa partes flexíveis dos pontos de visada do objeto. O dicionário é treinado usando um conjunto de dados que contém diferentes pontos de visada dos objetos onde só a forma é conhecida e o linha de visada não. Esse trabalho é muito relacionado com o trabalho de Zhu et al. (2008), só que eles não consideram a informação do linha de visada do objeto. Algo a ser destacado do fato de compartilhar as partes e aparência é que isso poderia simplificar o aprendizado requerendo menos treinamento.

Adicionalmente, existem trabalhos que além de usar a informação referente ao objeto em geral, 
consideram informação textual de atributos no treinamento como: tem perna, torso, cor, tais como em Wang e Mori (2010) e em Wang e Forsyth (2009).

Quando trata-se com vídeos, pode ser importante considerar a informação de fluxo óptico, ou descontinuidades de movimento, etc. Em Dalal (2006), introduz-se um esquema para deteç̧ão de pessoas em vídeos, em que as pessoas e a câmera podem estar estáticas ou em movimento. O esquema que ele apresenta se refere a descritores de características baseados em histogramas de orientações de diferenças locais ou diferenças do fluxo óptico (Motion HOG). Motion HOG usa um histograma de orientação, similar ao usado para os descritores HOG estáticos. Entretanto, ao invés de usar o gradiente da imagem, motion HOG usa diferenças do fluxo óptico, também orientações de fluxo ou gradientes espaciais orientados de componentes de fluxo. As características de movimento consideram duas imagens consecutivas de um vídeo como entrada. Neste caso, consideram-se usar duas imagens consecutivas, embora mais imagens proveem maior informação, isso implica num vetor maior no treinamento.

Da mesma forma, pelo fato de ter informação temporal, existem trabalhos que auxiliam-se da técnica de rastreamento ${ }^{1}$ (tracking) para melhorar a performance do processo de detecção (Andriluka et al., 2008; García-Martín e Martínez, 2012; Jain et al., 2011; Leibe et al., 2008b). No trabalho de Jain et al. (2011) usa-se rastreamento para acelerar o processo de deteç̧ão em grandes quantidades de vídeos. Onde o processo de rastreamento de um objeto é feito dado a localização do objeto em dois quadros, o primeiro e o último de uma tomada (shot) do vídeo, e interpolando as possíveis localizações do objeto nos quadros intermédios usando duas abordagens: gulosa (greedy) e programação dinâmica, mas tendo uma melhor performance com o método híbrido, assim como acelerar o processo de detecção. O principal problema é saber se realmente faz detecção do objeto em ambos quadros, porque caso não seja detectado o objeto ele não vai ser interpolado a traves dos quadros intermédios. No trabalho de García-Martín e Martínez (2012) integram-se um algoritmo de detecção de pessoas e as informações de rastreamento num único sistema, mostrando com os seus resultados obtidos que o uso de rastreamento estabiliza as detecções obtidas ao longo do tempo, então existe uma melhora em termos de revocação e pontuação $\mathrm{F}$.

Finalmente, neste trabalho escolheu-se as misturas de modelos de partes deformáveis (Felzenszwalb et al., 2010b) para a criação de modelos e para posteriormente aplicar ele na detecção de objetos em sequencias de vídeo. A descrição desses temas serão tratados nos próximos capítulos.

\footnotetext{
${ }^{1}$ Rastreamento refere-se ao processo de localizar um ou vários objetos no tempo (no vídeo).
} 


\section{Mistura de modelos baseados em partes deformáveis}

Neste capítulo, introduzimos os conceitos e os processos envolvidos no algoritmo proposto em Felzenszwalb et al. (2010b) e em Felzenszwalb et al., para a construção das misturas de modelos baseados em partes deformáveis (MDPMs) simétricas e assimétricas respectivamente, de uma classe de objeto.

O algoritmo para a construção das MDPMs, basicamente refere-se a um problema de aprendizado ou indução de um classificador, o qual no processo contém um passo de extração de características (descrito na Seção 3.1), a definição de uma MDPM (descrita na Seção 3.3), um processo de casamento (descrito na Seção 3.4) e da indução do classificador mesmo (descrita na Seção 3.5).

\subsection{Extração de características}

A extração de características refere-se às 31 características HOG que são obtidas para cada imagem/quadro (mapa de características). As 31 características HOG descrevem um objeto através da distribuição espacial das intensidades dos gradientes locais, computadas em células de pixels uniformemente espaçadas.

Para extrair os descritores de 31 características HOG de uma imagem $I$ de tamanho $w \times h$, são definidos uma série de passos:

- Calcular os gradientes $g_{x}$ e $g_{y}$ de $I$.

Os gradientes são calculados aplicando-se o vetor $[-1,0,1]$ e sua transposta (como o Sobel) para ter as gradientes nas dimensões $x$ e $y$ respectivamente.

- Dado os gradientes da imagem $I$, para cada canal de cor, defini-se $\theta(x, y)$ e $r(x, y)$ como a orientação e a magnitude da intensidade do gradiente no pixel $(x, y)$ respectivamente, onde:

$$
\begin{aligned}
& \theta(x, y)=\tan ^{-1}\left(\frac{g_{y}}{g_{x}}\right) \\
& r(x, y)=\sqrt{g_{x}^{2}+g_{y}^{2}} .
\end{aligned}
$$

Seleciona-se no processo só o canal de cor com maior magnitude do gradiente para cada pixel. As orientações dos gradientes de 0 a $\pi$ são definidas como insensiveis ao contraste ou "sem sinal", e as de $-\pi$ a $\pi$ como sensiveis ao contraste ou "com sinal". Se todas elas forem concatenadas para formar um descritor, gera-se um longo vetor de características sensível a pequenas deformações.

- Calculam-se os mapas de características dividindo $I$ em células não sobrepostas de comprimento $k \times k$, onde $k=8$.

Definem-se os mapas de características $C$ e $D$, onde $C$ contém vetores de características para 
as orientações sensíveis ao contraste e $D$ para as insensíveis ao contraste. Cada ponto $(i, j)$ de $C$ e $D$, é definido por um histograma de $q$ intervalos com os valores das magnitudes dos pixels $(\lfloor x / k\rfloor,\lfloor y / k\rfloor)$ de $I$ como segue:

- Discretiza-se a orientação de cada pixel de $I$ em um intervalo definido por $B_{i}$, onde $B_{i}$ é igual a $B_{1}$ ou $B_{2}$, os quais são definidos como:

$$
\begin{aligned}
& B_{1}(x, y)=\text { round }\left(\frac{q * \theta(x, y)}{2 \pi}\right) \bmod q, \\
& B_{2}(x, y)=\text { round }\left(\frac{q * \theta(x, y)}{\pi}\right) \bmod q,
\end{aligned}
$$

onde para $B 1$ (orientações sensíveis ao contraste) tem-se 18 intervalos, portanto usa-se $q=18$ e para $B_{2}$ (orientações insensíveis ao contraste) tem-se 9 intervalos, portanto usase $q=9$. Desta forma, os intervalos estão definidos cada 20. Por exemplo, um gradiente com uma orientação de $-\pi / 4$, será adicionada no intervalo número 2 em $B_{2}$ e no intervalo número 16 em $B_{1}$.

- Logo cada mapa de características ao nível de células é dado por:

$$
C(i, j)_{p}=C(i, j)_{p}+\operatorname{val}(\lfloor x / k\rfloor,\lfloor y / k\rfloor),
$$

assim, $p \in\{0, \ldots, q-1\}$ é um intervalo de orientações definido por $B_{i}$. Isto é $B_{i}$ é igual a $B_{1}$ para $C$ e $B_{2}$ para $D$. Adicionalmente, o valor de $\operatorname{val}(\lfloor x / k\rfloor,\lfloor y / k\rfloor) \in C(i, j)$ é dado por:

$$
\operatorname{val}(\lfloor x / k\rfloor,\lfloor y / k\rfloor)= \begin{cases}r(\lfloor x / k\rfloor,\lfloor y / k\rfloor), & \text { se } p=B_{i}(\lfloor x / k\rfloor,\lfloor y / k\rfloor), \\ 0, & \text { caso contrário }\end{cases}
$$

Desta forma, o vetor de características da célula é definido como a soma das magnitudes dos pixels nessa célula, conseguindo desta forma a invariância em pequenas deformações e reduzindo, por sua vez, o tamanho do mapa de características. Um representação da construção de uma célula (região com borda de cor azul) em $C$ e $D$ é mostra-se na Figura 3.1.

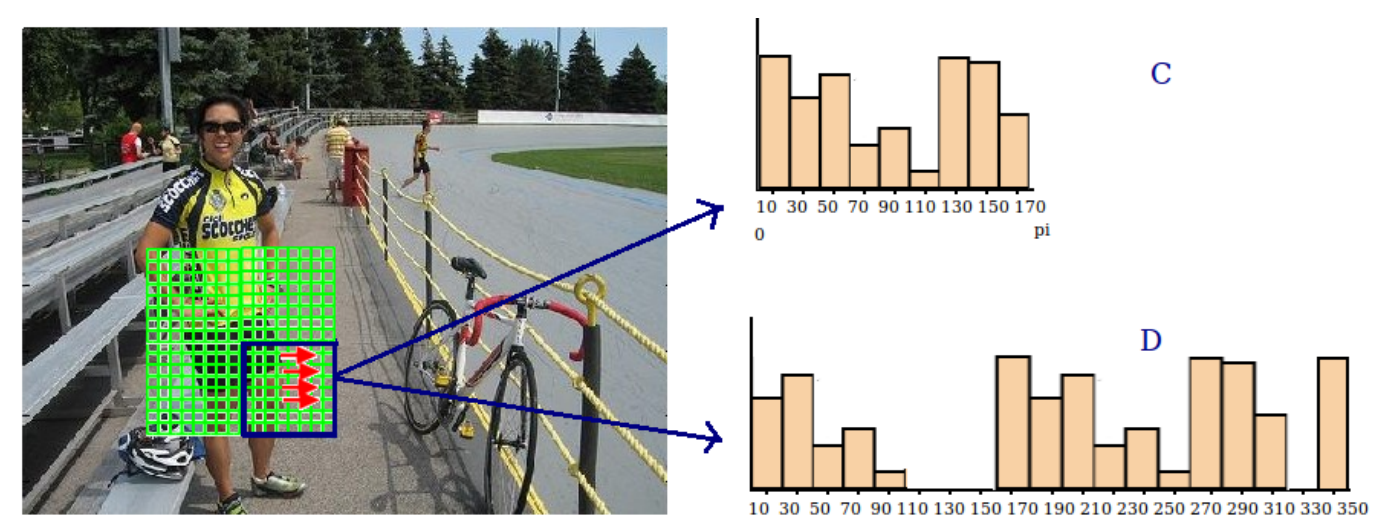

Figura 3.1: Uma representação de como fica o histograma de uma célula de $C$ e D.

\section{- Em seguida, o vetor de características de cada célula de $C$ e $D$ é normalizado e truncado.}

Aplica-se a Equação (3.5), respectivamente para $C$ e $D$ criando mapas de características $H_{m_{C}}$ 
e $H_{m_{D}}$ respectivamente.

$$
H_{m_{C}}(i, j)=\left(\begin{array}{c}
T_{\alpha}\left(C(i, j) / N_{-1,-1}(i, j)\right) \\
T_{\alpha}\left(C(i, j) / N_{+1,-1}(i, j)\right) \\
T_{\alpha}\left(C(i, j) / N_{+1,+1}(i, j)\right) \\
T_{\alpha}\left(C(i, j) / N_{-1,+1}(i, j)\right)
\end{array}\right)
$$

onde $N_{\delta, \gamma}(i, j)=\sqrt{\left(\|C(i, j)\|^{2}+\|C(i+\delta, j)\|^{2}+\|C(i, j+\gamma)\|^{2}+\|C(i+\delta, j+\gamma)\|^{2}\right)}$, com $\delta, \gamma \in\{-1,1\}$, mede a norma do gradiente em um bloco quadrado de quatro células contendo $(i, j) . T_{\alpha}(v)$ representa a função de truncamento do vetor $v$ com $\alpha$. Desta forma, o $p$-ésimo intervalo em $T_{\alpha}(v)$ é o mínimo entre o $p$-ésimo intervalo de $v$ e $\alpha$, com $\alpha=0.2$.

- Define-se o mapa de características HOG, $H_{m}$, pela concatenação de $C$ e $D$ normalizados, isto é, $H_{m}=\left[H_{m_{C}} H_{m_{D}}\right]$.

Note que com a concatenação, a dimensão de cada descritor do mapa de características HOG é de $4 *(9+18)=108$ dimensões.

- Finalmente, aplica-se sob o vetor 108-dimensional uma transformação chamada de projeção analítica, de onde obtém-se o vetor final 31-dimensional, que representa o vetor de características HOG em $(i, j)$.

A projeção analítica é definida por 27 somas sob as normalizações diferentes, isto é, para cada intervalo de orientação (9 insensíveis ao contraste e 18 sensíveis ao contraste) soma-se os valores obtidos para cada fator de normalização. Adicionalmente, por 4 somas sob as 9 orientações insensíveis ao contraste, uma para cada fator de normalização. Esses 4 valores das somas são denominadas como as gradientes de texture. A Figura 3.2 mostra o mapa de características ao nível de um vetor de características HOG-31 dimensional.

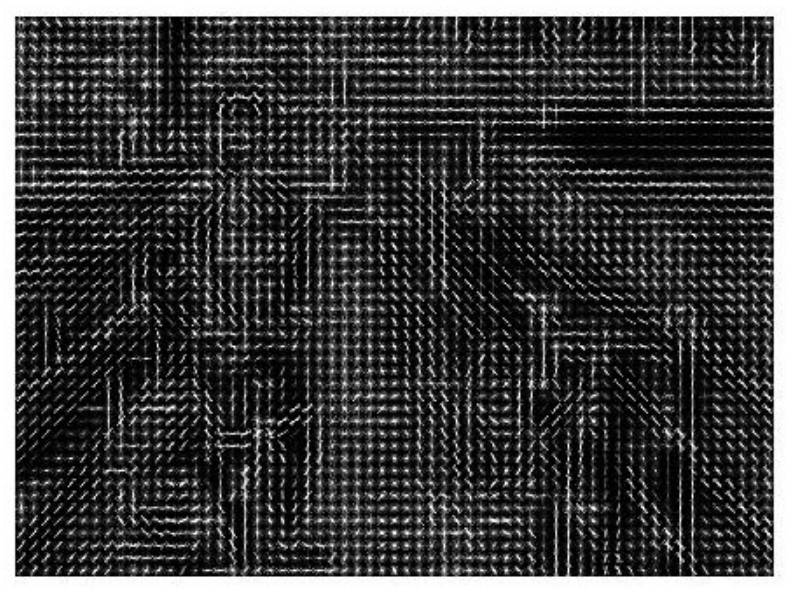

Figura 3.2: Representação do mapa de características HOG-31 dimensional.

\subsection{Filtro e Pirâmide de características}

Os modelos deformáveis são construídos através de características HOG e de operações de filtragem. Neste caso, um filtro é um padrão retangular definido em um mapa de características ao nível de 31 características HOG, mas usaremos o termo de filtro para enfatizar que atuam como pesos nas características em vez de características.

Cria-se uma pirâmide de mapas de características HOG $\left(H=\left(H_{1}, \ldots, H_{s}\right)\right)$, através do borramento e subamostragem de uma imagem original e calculando o mapa de características HOG para cada nível $\left(s_{i}\right)$ da pirâmide. Dois filtros chamados raiz e parte são definidos em $H$ (veja a 
Figura 3.3). O filtro raiz refere-se à representação "bruta" (coarse) dos objetos, enquanto que os filtros parte referem-se a uma representação mais detalhada pois é obtido ao dobro de resolução no qual foi obtido o filtro raiz. Os filtros parte são calculados descendo $\lambda$ níveis na pirâmide desde o nível do filtro raiz, ou seja, o nível do filtro raiz é $l_{0}$ e o nível dos filtros parte é $l_{i}=l_{0}-\lambda$. Usualmente, $\lambda=5$ no processo de treinamento e $\lambda=10$ no teste (Felzenszwalb et al., 2010b).

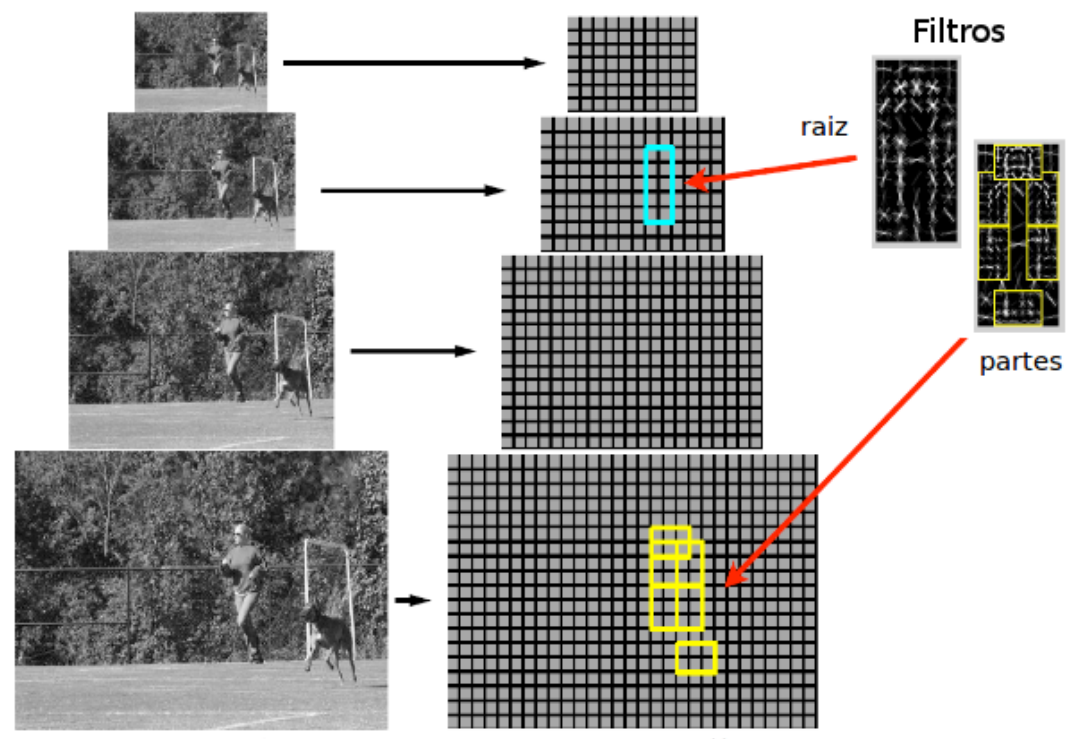

Figura 3.3: Representação da pirâmide de características HOG-31 dimensional e a definição dos filtros nela. Imagem adaptada de Felzenszwalb et al. (2010b).

\subsection{Definição da MDPM}

Denotemos por $M$ uma mistura de modelos definida por $m$ componentes $\left(M_{1}, \ldots, M_{m}\right)$. O modelo para a $c$-ésima componente $\left(M_{c}\right)$ é baseado em um modelo estrela de uma estrutura pictórica, ou seja, ele é definido por filtros lineares (filtro raiz e filtros parte); por um conjunto de localizações permitidas para cada parte com relação à raiz; e um custo de deformação para cada parte. Formalmente, o modelo da $c$-ésima componente da mistura consiste de uma $(n+2)$-tupla definida como:

$$
M_{c}=\left(F_{0}, P_{1}, \ldots, P_{n}, b\right),
$$

onde $F_{0}$ é o filtro raiz (veja a Figura 3.4a), $n$ é o número de partes (da Figura 3.4b temos $n=5$ ), $P_{i}$ é o modelo parte $i$. Cada modelo parte é definido por uma 3-tupla $\left(F_{i}, v_{i}, d_{i}\right)$, onde $F_{i}$ é o $i$-ésimo filtro parte, $v_{i}$ indica uma localização fixa para a parte $i$ em relação à posição da raiz e $d_{i}$ indica os parâmetros de uma função do custo de deformação para cada localização da parte $i$ em relação a $v_{i}$. O $b$ representa o viés, um valor real que faz às pontuações de modelos múltiplos comparáveis quando eles são combinados em mistura de modelos.

Vejamos com maior detalhe a definição dos vetores $v_{i}$ e $d_{i}$ da 3 -tupla $\left(F_{i}, v_{i}, d_{i}\right)$ :

- $v_{i}$ é um vetor bidimensional que indica uma localização fixa para a parte $i$ em relação à posição da raiz. Da Figura 3.4c temos o vetor $v_{3}$, de cor cyan, que indica a localização fixa da parte 3 com respeito à raiz.

- $d_{i}$ é um vetor quadridimensional que indica os parâmetros ou coeficientes de uma função quadrática que define um custo de deformação para cada localização da parte $i$ em relação a $v_{i}$. Da Figura 3.4d observa-se como estão deslocadas as partes logo de um processo de casamento entre o modelo e uma instância numa imagem. Então, as características de deformação neste caso estão dados pela função quadrática: $\phi_{d}\left(d x_{i}, d y_{i}\right)=\left(d x, d y, d x^{2}, d y^{2}\right)$, onde $\left(d x_{i}, d y_{i}\right)=$ $\left(x_{i}, y_{i}\right)-\left(2\left(x_{0}, y_{0}\right)+v_{i}\right)$ e representa o deslocamento da parte, logo se, por exemplo, os 


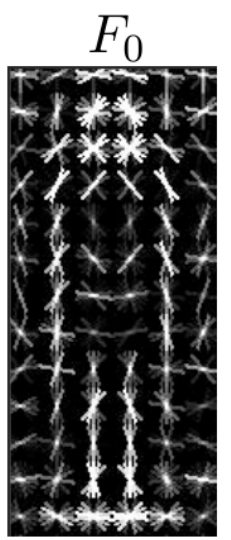

(a)
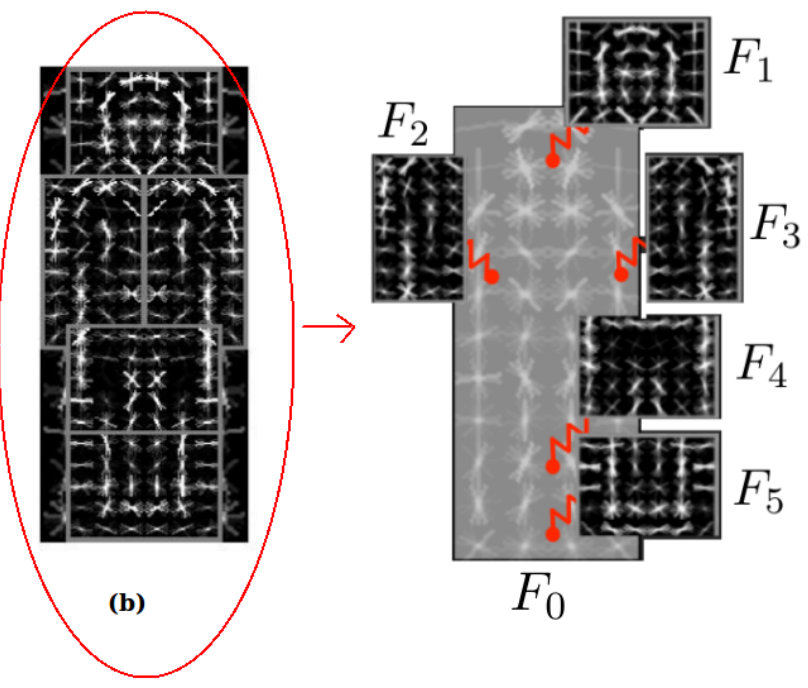

(c)

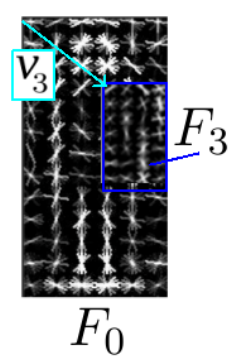

(d)

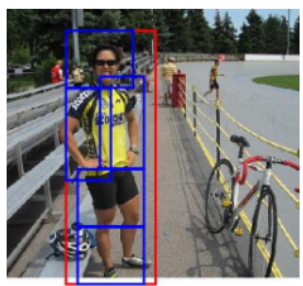

Figura 3.4: Definição da MDPM. (a) Filtro raiz, (b) filtros parte de uma MDPM, (c) localização do filtro $F_{3}\left(v_{3}\right)$ e (d) exemplo de deformação das partes.

coeficientes da função quadrática, $d_{i}$, são $(0,0,1,1)$, então temos que o custo de deformação para a parte $i$ é o quadrado da distância entre a posição atual e a posição fixa em relação à raiz.

\subsubsection{Hipótese objeto}

Uma hipótese objeto $h_{o b j}$ especifica uma $c$-ésima componente da mistura e as localizações do filtro raiz e filtros parte na pirâmide de características $H$. Formalmente, a hipótese objeto é definida como:

$$
h_{o b j}=\left(c, p_{0}, \ldots, p_{n}\right),
$$

onde $p_{i}=(x, y, l)$ representa a posição $(x, y)$, e o nível $l$ na pirâmide $H$ para o filtro $i$.

\subsection{Processo de casamento}

Em Felzenszwalb et al. (2010b), abordou-se o problema de deteç̧ão de objetos em imagens com uma análise de casamento por janelas deslizantes usando misturas de modelos multi-escala de partes deformáveis (MDPMs) para cada classe. Para o casamento de uma estrutura pictórica-estrela com uma imagem, usa-se programação dinâmica e transformada da distância generalizada. Isto permite uma busca eficiente em todas as possíveis configurações do objeto na imagem sem restringir as possíveis localizações de cada parte.

O processo de casamento consiste em definir uma pontuação para cada localização do filtro raiz baseada na melhor localização das partes de maneira independente para cada componente. Assim, uma alta pontuação de localização da raiz define uma detecção. Formalmente, o processo de deteç̧ão ou casamento é dado por:

$$
\operatorname{score}\left(p_{0}\right)=\max _{p_{1}, \ldots, p_{n}} \operatorname{score}\left(h_{o b j}\right),
$$

em que a pontuação da hipótese objeto, $\operatorname{score}\left(h_{o b j}\right)$, é dada pela pontuação dos filtros, e suas localizações, menos um custo de deformação que depende da posição relativa de cada parte com respeito do filtro raiz, mais o viés. Formalmente, a pontuação da hipótese objeto é definida como:

$$
\operatorname{score}\left(h_{o b j}\right)=\sum_{i=0}^{n} F_{i} . \phi\left(H, p_{i}\right)-\sum_{i=1}^{n} d_{i} \cdot \phi_{d}\left(d x_{i}, d y_{i}\right)+b,
$$


onde $\phi\left(H, p_{i}\right)$ é uma sub-janela na pirâmide $H$ com o canto superior esquerdo em $p_{i}=(x, y, l)$, $\phi_{d}\left(d x_{i}, d y_{i}\right)=\left(d x, d y, d x^{2}, d y^{2}\right)$ é a função quadrática das características de deformação, e $d_{i}$ são os coeficientes de uma função quadrática (tal como foi visto anteriormente).

Na prática, o algoritmo de casamento começa calculando a resposta do filtro $i$ no nível $l$ como:

$$
R_{i, l}(x, y)=F_{i} . \phi\left(H, p_{i}\right) .
$$

Em seguida são transformados os $R_{i, l}$ dos filtros parte para calcular a máxima contribuição da parte $i$ à pontuação da localização da raiz. Essa transformação é dada por:

$$
D_{i, l}(x, y)=\max _{d x, d y}\left(R_{i, l}(x+d x, y+d y)-d_{i} \phi_{d}(d x, d y)\right) .
$$

Finalmente, a pontuação de localização do filtro raiz, definida na Equação (3.8), pode ser reescrita como:

$$
\operatorname{score}\left(p_{0}\right)=R_{o, l_{0}}\left(x_{0}, y_{0}\right)+\sum_{i=1}^{n} D_{i, l_{i}}\left(2\left(x_{0}, y_{0}\right)+v_{i}\right)+b .
$$

O método leva para um tempo de $O(n s)$ ao calcular a resposta de um filtro, onde $n$ é o número de partes e $s$ é o número total de localizações na pirâmide (Felzenszwalb et al., 2010b).

\subsection{Indução do classificador}

A indução do classificador refere-se ao processo de treinamento das MDPMs. O classificador precisa aprender a estrutura da MDPM, os filtros e os custos de deformação. Para o processo de aprendizado, é usada SVM linear e SVM latente (formulação definida em Felzenszwalb et al. (2008)).

A seguir, são descritos, de maneira geral, a formulação do SVM latente e os passos envolvidos no treinamento das MDPMs simétricas e assimétricas. A formulação do SVM latente é baseada na definição do SVM linear mas considerando variáveis latentes. Veja o Apêndice A para uma introdução do SVM linear.

\subsubsection{SVM latente}

Seja o conjunto de dados de treinamento $T=\left\{\left(\mathbf{x}_{1}, y_{1}\right), \ldots,\left(\mathbf{x}_{m}, y_{m}\right)\right\}$, em que $\mathbf{x}_{i} \in \mathbb{R}^{n}$ e $y \in$ $\{-1,1\}$. Seja uma variável latente $z \in Z(\mathbf{x})$ (um conjunto de variáveis latentes possíveis para um exemplo $\mathbf{x}$ ). Seja a função linear, que deseja-se aprender (o classificador), definida como:

$$
f(\mathbf{x})=\max _{z \in Z(\mathbf{x})} \mathbf{w} \cdot \Phi(\mathbf{x}, z),
$$

onde w e $\Phi(\mathbf{x}, z)$ são vetores $\in \mathbb{R}^{n}$.

A função objetivo do SVM latente é definida por:

$$
\min _{\mathbf{w}} \frac{1}{2}\|\mathbf{w}\|^{2}+C \sum_{i=1}^{m} \max \left(0,1-y_{i} f\left(\mathbf{x}_{i}\right)\right),
$$

de onde pode-se observar que ao restringir o domínio de $Z\left(\mathbf{x}_{i}\right)$ para uma única escolha, $f(\mathbf{x})$ torna-se linear em $\mathbf{w}$ obtendo assim um SVM linear como um caso especial dos SVMs latentes.

O SVM latente resulta em um problema de otimização não convexo, devido ao max introduzido em $f(\mathbf{x})$. No entanto, Felzenszwalb et al. (2010b) mostrou que o SVM latente é semi-convexo e observou que fixando os valores latentes $z$ para os exemplos positivos na Equação (3.13), ele é 
convexo. Ou seja, a Equação (3.13) é convexa em w porque é a função máxima de um conjunto de funções lineares e a função de perda $\max \left(0,1-y_{i} f\left(\mathbf{x}_{i}\right)\right)$ é convexa pois:

- Para exemplos negativos $\left(y_{i}=-1\right)$, a função $1+f\left(\mathbf{x}_{i}\right)$ é convexa. Esta propriedade da função de perda é conhecida como semi-convexidade.

- Para exemplos positivos $\left(y_{i}=1\right)$, a função $1-f\left(\mathbf{x}_{i}\right)$ é côncava. Mas se $Z\left(\mathbf{x}_{i}\right)$ é restrito para uma única escolha, então $f\left(\mathbf{x}_{i}\right)$ é linear. Logo, a função de perda é convexa.

Desta forma, combinando as propriedades de convexidade e semi-convexidade temos que a Equação (3.14) é convexa em w. Logo, para a solução da função objetivo, usa-se o algoritmo de coordenada descendente, com o compromisso de encontrar os melhores valores latentes e otimizar a função objetivo do SVM latente, ou seja:

- Dado $\mathbf{w}$, para cada exemplo positivo $\mathbf{x}_{i}$, encontrar o $z_{i}$ que maximize: w. $\Phi(\mathbf{x}, z)$.

- Dado um $z_{i}$ de um exemplo positivo, encontrar o w que otimize a função objetivo do SVM latente.

Nos dois passos envolvidos no algoritmo de coordenada descendente, pode-se observar um ciclo. Para quebrar o ciclo, assume-se as localizações dos exemplos de treinamento como as localizações iniciais $z_{o}$ das características.

\subsubsection{SVM latente para as MDPMs}

No problema das MDPMs, para se realizar o aprendizado dos parâmetros das componentes da MDPM com a definição do SVM latente, a pontuação da hipótese objeto $h_{o b j}$ deve ser redefinida como:

$$
\begin{aligned}
\operatorname{score}\left(h_{o b j}\right) & =\sum_{i=0}^{n} F_{i} . \phi\left(H, p_{i}\right)-\sum_{i=1}^{n} d_{i} \cdot \phi_{d}\left(d x_{i}, d y_{i}\right)+b, \\
& =\underbrace{\left(F_{0}, \ldots, F_{n}, d_{1}, \ldots, d_{n}, b\right)}_{\beta} \cdot \underbrace{\left(\phi\left(H, p_{0}\right), \ldots, \phi\left(H, p_{n}\right),-\phi_{d}\left(d x_{1}, d y_{1}\right), \ldots,-\phi_{d}\left(d x_{n}, d y_{n}\right), 1\right)}_{\psi\left(H, h_{o b j}\right)}, \\
& =\beta . \psi\left(H, h_{o b j}\right) .
\end{aligned}
$$

Logo, na formulação do SVM Latente, a função linear que deseja-se aprender (Equação (3.13)) fica como:

$$
f(\mathbf{x})=\max _{z \in Z(\mathbf{x})} \beta \cdot \psi\left(H, h_{o b j}\right)
$$

onde cumpre-se o seguinte:

- Os dados de treinamento consistem de imagens em bounding boxes anotados, e um exemplo positivo define o objeto de uma classe e um exemplo negativo é criado usando sub-janelas aleatórias das imagens que não contém o objeto procurado. Na Figura 3.5 pode-se observar exemplos positivos e negativos para o objeto carro, onde os exemplos positivos são imagens de carros com "bounding boxes" e os negativos são imagens sem carros.

- Para exemplos positivos, $x_{i}=\left(I_{x_{i}}, B_{x_{i}}\right)$, onde $I_{x_{i}}$ é uma imagem positiva e $B_{x_{i}}$ é o bounding box para uma instância da classe alvo em $I_{x_{i}}$.

- Para exemplos negativos, $x_{i}=\left(I_{x_{i}}, l_{x_{i}}^{(1)}, \ldots, l_{x_{i}}^{(m)}\right)$, onde $I_{x_{i}}$ é uma imagem negativa e $l_{x_{i}}^{(c)}$ é a localização do filtro raiz para a $c$-ésima componente. Essas localizações são usadas para colocar os filtros raiz nessas localizações e fazer coincidir os seus centros geométricos.

- As variáveis latentes $Z\left(x_{i}\right)$ são definidos por uma etiqueta na componente da mistura e por um conjunto de posições válidos para o filtro raiz e os filtros de partes. 

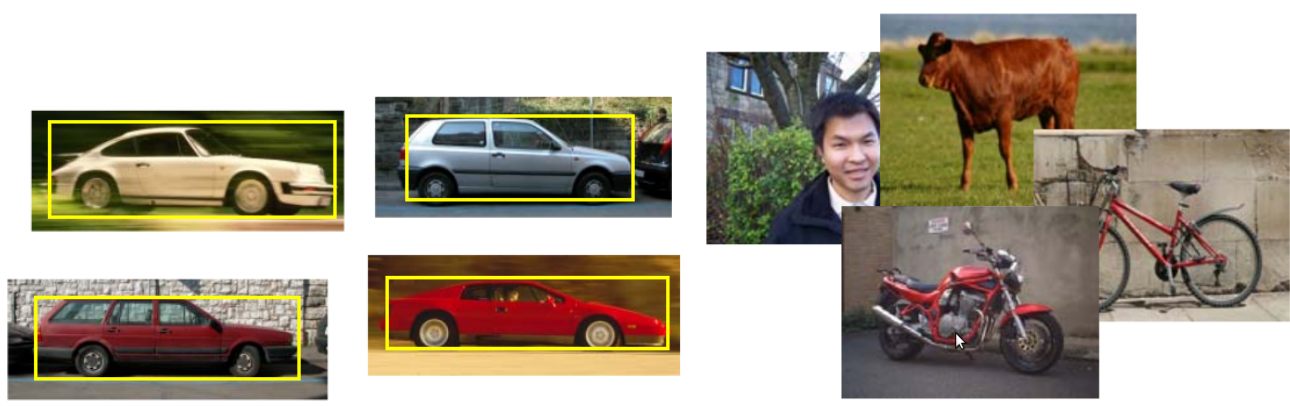

Figura 3.5: Exemplos positivos e negativos para a classe do objeto carro.

- Para exemplos positivos, o conjunto $Z\left(x_{i}\right)$ é definido pelas localizações do filtro raiz com uma sobreposição de $70 \%$ ou mais com o bounding box.

- Para exemplos negativos, no conjunto $Z\left(x_{i}\right)$, a etiqueta da componente e as localizações da raiz são restritas só para o conjunto $\left\{\left(1, l_{x_{i}}^{(1)}\right), \ldots,\left(m, l_{x_{i}}^{(m)}\right)\right\}$.

\subsubsection{Treinamento das MDPMs simétricas}

O processo de treinamento recebe como entrada o conjunto de exemplos positivos e negativos. Os exemplos positivos são ordenados pela relação de aspecto ${ }^{1}$ dos bounding boxes e divididos em $n c$ grupos de tamanho idêntico, onde $n c$ é o número de componentes da mistura. Assim, aqueles exemplos com relação de aspecto dos bounding boxes similares pertencem ao mesmo grupo.

Inicializa-se o filtro raiz (o modelo inicial) para cada grupo de exemplos positivos. Para isso:

- Defini-se a dimensão de cada filtro pela dimensão da média entre as relações de aspecto e a maior área não maior que $80 \%$ dos bounding boxes.

- Recortar e escalar os exemplos positivos à dimensão definida.

- As características HOG são obtidas para cada exemplo e o filtro é treinado usando uma SVM linear, sem informação latente, considerando exemplos negativos aleatórios.

- Os filtros raiz são forçados a serem simétricos com relação ao seu eixo vertical.

Após ter o filtro raiz treinado para cada grupo, todos eles são combinados em uma mistura sem partes e os parâmetros do modelo são treinados usando as imagens originais (sem transformação de escala) e uma SVM latente. A variável latente é a localização do filtro raiz e a componente da mistura para cada exemplo positivo. Desta forma, para cada exemplo positivo, é estimado um bounding box latente com sobreposição maior que $70 \%$ com o bounding box original. Por exemplo, na Figura 3.6 o bounding box original é aquele de cor amarela e o bounding box latente é aquele de cor ciano.

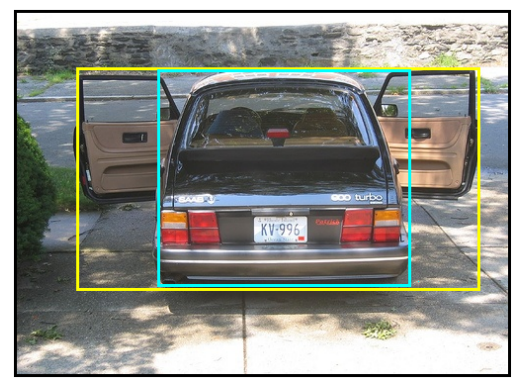

Figura 3.6: Exemplo de bounding box latente.

\footnotetext{
${ }^{1}$ A relação de aspecto (aspect ratio) de uma imagem, é a relação que existe entre a sua largura e altura.
} 
Após a construção do filtro raiz para cada componente da mistura, emprega-se uma heurística simples para inicializar os filtros partes de cada componente:

- Fixa-se o número de partes das componentes para: $n p_{c}$.

- Seleciona-se uma área $a$ tal que $n p_{c} . a$ seja o maior que $80 \%$ da área do filtro raiz.

- Usando um processo guloso com uma heurística simples, inicializa-se cada uma das $n p_{c}$ partes com a região retangular do filtro raiz de área $a$ que possui a maior energia positiva. A energia de uma região é definida pela norma dos pesos positivos em uma sub-janela. Quando uma parte é fixada, a energia da região coberta do filtro raiz é definida por zero e procura-se a seguinte região com maior energia positiva.

- Os filtros raiz são inicializados por uma interpolação da região do filtro raiz coberta por cada parte ao dobro de resolução do filtro raiz.

- Os filtros parte que estão centrados no eixo vertical de um filtro raiz são verticalmente simétricas, e os que não estão centrados são verticalmente refletidos.

- Inicializa-se os custos de deformação $d_{i}$, ou seja os custos de deformação para cada parte. Eles medem a norma ao quadrado do deslocamento, isto é: $d_{i}=(0,0,0.1,0.1)$, dado que esses valores mostraram melhores resultados.

- Depois, usa-se um processo de busca local para mover as partes, uma à vez, numa ordem aleatória para maximizar a energia do filtro raiz coberta. Caso uma melhor cobertura de energia não seja encontrada, reinicializa-se à configuração inicial e escolhe-se outra ordem de relocalização das partes. Finalmente, depois de várias repetições do processo, é selecionada a configuração das partes que apresentaram uma maior energia coberta.

Finalmente, a mistura de modelos é atualizada treinando-o com novos dados e considerando a componente, o filtro raiz, filtros parte e localizações das partes. Para isso:

- Tem-se que construir novos dados de treinamento para atualizar os modelos, isto é, aplicase o detector existente em todas as posições e escalas. Seleciona-se o de maior pontuação dentre aqueles com pelo menos $50 \%$ de sobreposição com cada bounding box dos dados de treinamento. Logo, exemplos negativos hard são adicionados em uma memória cachê até os limites de tamanho do arquivo.

Os exemplos negativos hard são aqueles que foram classificados como positivos. Eles são mantidos para o treinamento, pelo fato de poder ter uma quantidade de exemplos negativos que torne o algoritmo de otimização impossível de ser resolvido.

- Um novo modelo é treinado executando SVMLight Joachims (1999b) nos exemplos positivos e negativos hard da memória cachê, cada um deles etiquetados com as posições das partes.

A Figura 3.7 mostra uma MDPM assimétrica de 3 componentes e 6 partes do modelo para a classe carro. Cada linha representa uma componente, a primeira coluna mostra o filtro raiz, a segunda coluna mostra os filtros parte e a terceira coluna mostra o modelo espacial para cada parte.

\subsubsection{Treinamento das MDPMs assimétricas}

O autor criou inicialmente modelos simétricos para as classes, seguindo os processos descritos anteriormente. Em seguida, propôs criar modelos assimétricos, os quais referem-se à criação de componentes tanto para a pose do lado esquerdo como para o lado direito. Para isso, ele adiciona um passo de agrupamento (clustering) de dois grupos (esquerdo e direito) os quais são simétricos entre eles. A distinção das poses é feita automaticamente sem supervisão.

Desta forma, seguindo os passos descritos para o treinamento das MDPMs simétricas, depois de dividir o conjunto de imagens em grupos considerando a relação de aspecto, cada um desses grupos 

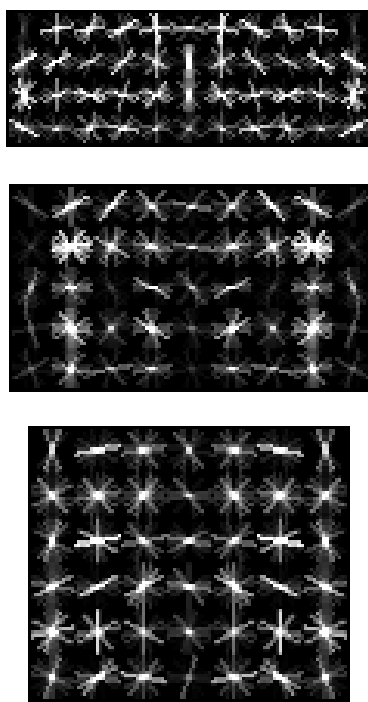
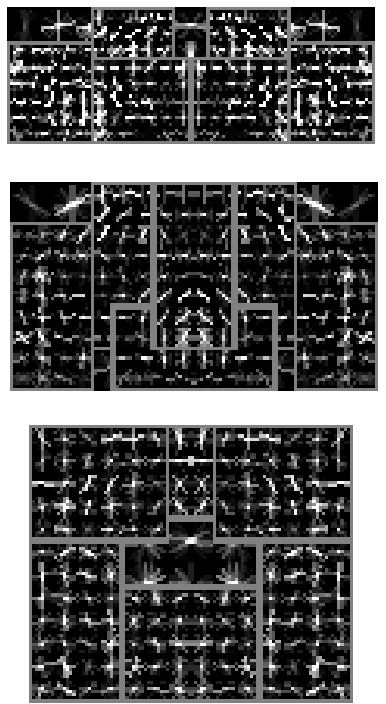
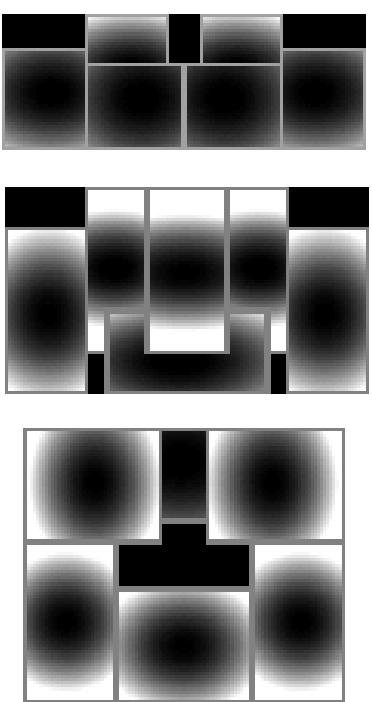

Figura 3.7: A MDPM simétrica de 3 componentes e 6 partes do modelo.

é agrupado de acordo com a pose em direito e esquerdo. Para agrupar de acordo com a pose, cada exemplo é recortado e é refletido no espaço de características, obtendo assim para cada exemplo um outro exemplo que é seu reflexo. Desta forma, o conjunto de imagens positivas de cada grupo é duplicada.

O algoritmo de agrupamento é uma variante do algoritmo k-médias, com a restrição de que um exemplo e seu reflexo não pertencem a um mesmo grupo. Aleatoriamente e repetitivamente um exemplo e seu reflexo são selecionados e atribuídos para um grupo. Em seguida que todos os exemplos foram atribuídos para um grupo, um método de busca local é usado para melhorar o agrupamento. Esta busca local é feita selecionando-se repetidamente um exemplo e seu reflexo e verificando se trocando eles de grupo, a soma das distâncias ao quadrado (SSD) dos exemplos para o centro de seu grupo é reduzida. O processo de agrupamento é repetido várias vezes utilizando diferentes exemplos iniciais para evitar mínimos locais. O grupo com o melhor valor da função objetivo SSD é selecionado.

Os passos seguintes são similares aos descritos para as MDPMs simétricas, omitindo os passos para a construção dos filtros simétricos. A Figura 3.8 mostra uma MDPM simétrica de 3 componentes e 6 partes do modelo para a classe carro. A MDPM assimétrica final tem 6 componentes por causa da assimetria bilateral que permite a cada componente representar as poses direita e esquerda, como pode ser visto nas linhas ímpar e par respectivamente. Por outro lado, neste caso estamos considerando cada duas linhas uma componente. Finalmente, temos que a primeira coluna mostra o filtro raiz, a segunda coluna mostra os filtros parte e a terceira coluna mostra o modelo espacial para cada parte. 

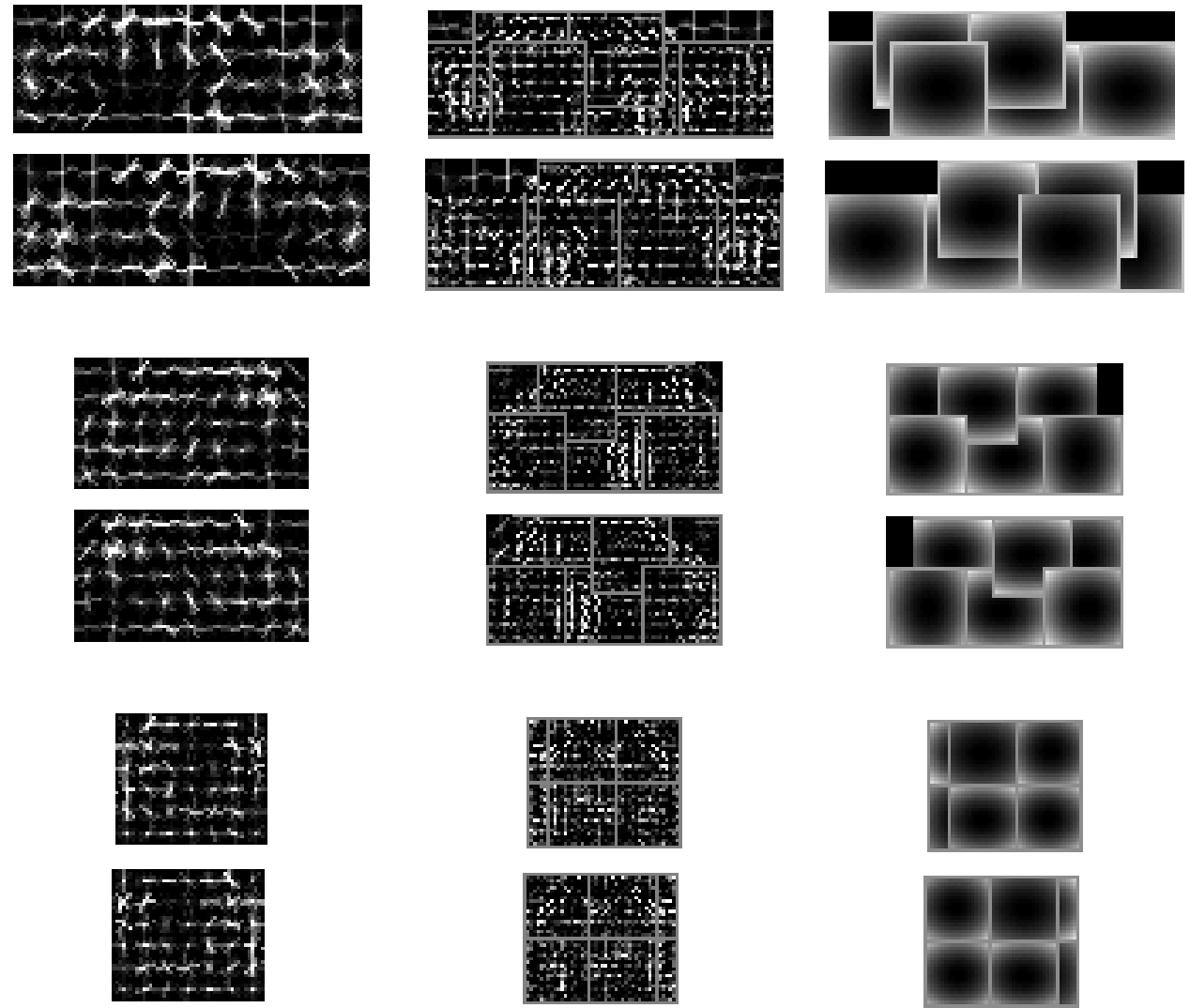

Figura 3.8: A MDPM assimétrica de 3 componentes e 6 partes do modelo. 


\section{Detecção de objetos em vídeos}

Neste capítulo descrevemos o método proposto para tratar o problema de detecção de objetos em vídeos. O problema é dividido em dois sub-problemas: criação de modelo do objeto (treinamento) usando um conjunto de imagens (descrito na Seção 4.1) e em aplicar o modelo nos vídeos, o qual é descrito na Seção 4.2, assim como os diferentes conjuntos de vídeos usados no trabalho.

\subsection{Criação de modelos}

Para o processo de criação de modelos usou-se as misturas de modelos baseados em partes deformáveis (MDPMs), técnica descrita no capítulo anterior. Usou-se um conjunto de dados de imagens no treinamento. Lembrando que o nosso objetivo é gerar modelos afins com o mundo, então foi escolhido um conjunto de dados que melhor representasse o objeto no mundo real.

Na literatura, encontram-se disponíveis diferentes conjuntos de dados para as tarefas de classificação e detecção de objetos. No trabalho de Torralba e Efros (2011) usando os dados de treinamento de 5 conjuntos de imagens, treina-se um classificador usando uma SVM e diferentes descritores. Em seguida, testa-se cada classificador com os dados de teste de todos os conjuntos de imagens (crossdataset), obtendo maior performance quando o conjunto de teste pertence ao mesmo conjunto de imagens do treinamento. Isso implica, segundo Torralba e Efros (2011), que os conjuntos de dados usualmente estão definidos por um bias.

O fato de um conjunto de dados estar definido por um bias significa que são criados para trabalhar sob um certo contexto definido pela tipo de cena, pelas localizações dos objetos nas imagens, pela linha de visada do objeto, entre outros. Por exemplo da Figura 4.1 temos exemplos de carros recortados de diferentes conjuntos de dados. Da figura, observa-se que existem algumas diferenças entre os diferentes conjuntos de imagens: Caltech tem preferência por carros com pontos de visada de lado; ImageNet por carros de corrida; PASCAL por conter carros com diferente linha de visada e de diversos tipos de carros; SUNS e LabelMe consideram também diferentes pontos de visada mas não há muita variedade de tipos de carros; adicionalmente, em LabelMe usualmente os objeto de interesse estão ocluídos por outros objetos.

Desta forma, tem-se a pergunta: quão bem um detector de objetos treinado com um determinado conjunto de dados generaliza quando é testado com outro conjunto de dados, comparado com sua performance no seu conjunto de teste original? Segundo Torralba e Efros (2011), o PASCAL e o ImageNet são os conjuntos de dados que melhor auxiliam na generalização, isto é, que melhor representam o mundo real. Assim, usamos o conjunto de dados PASCAL, o qual é descrito na Subseção 4.1.1. Isto é o que nos diferencia do existente na literatura, dado que a maioria dos trabalhos usam imagens onde o objeto encontra-se numa determinada linha de visada, ou ocupando a maior parte da imagem. 


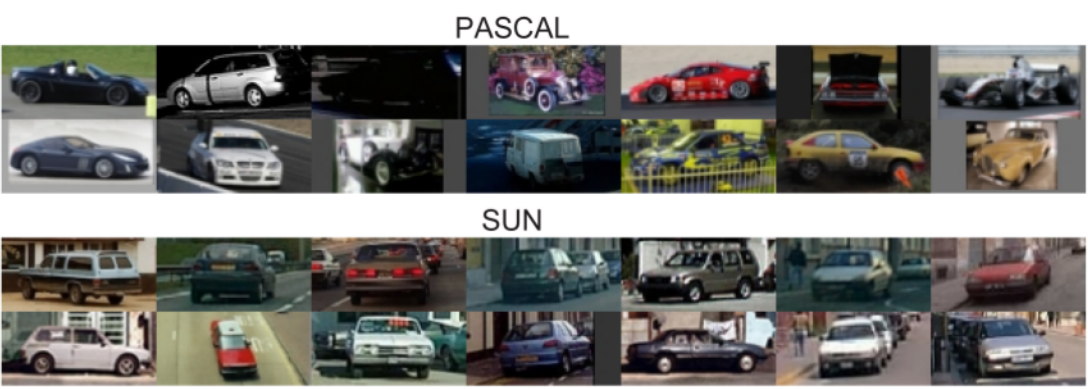

Caltech101

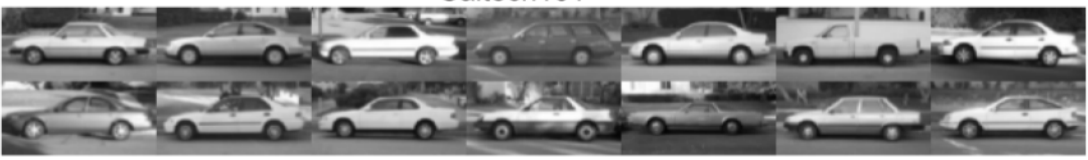

ImageNet

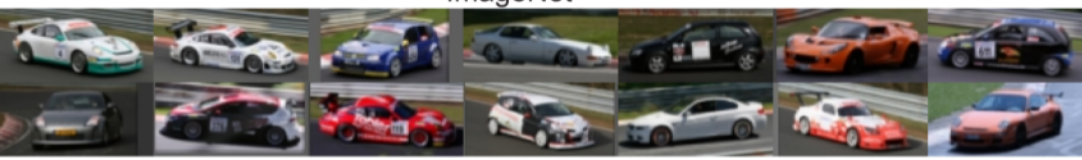

LabelMe

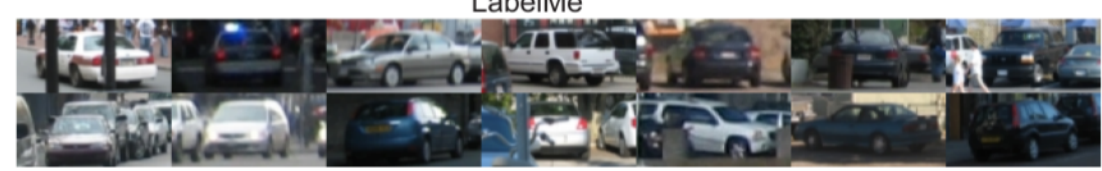

Figura 4.1: Exemplo de carros mais discriminativos de diferentes conjuntos de dados. Reproduzida de Torralba e Efros (2011).

\subsubsection{Conjunto de dados de imagens}

O desafio PASCAL VOC (Visual Object Classes) proporciona às comunidades de visão e aprendizado de máquina um conjunto de dados de imagens e bounding boxes, além de procedimentos padrão de avaliação. Anualmente, desde 2005, desafios são organizados e os conjuntos de dados associados a eles tornaram-se aceitos como pontos de referência para detecção de objetos (Everingham et al., 2010).

Neste trabalho foram usados os conjuntos de dados do PASCAL VOC 2007. Nestes conjuntos, as imagens estão categorizada em 20 classes de objetos: "pessoa", "passarinho", "gato", "vaca", "cão", "cavalo", "ovelha", "avião", "bicicleta", "barco", "ônibus", "carro", "motocicleta", "trem", "garrafa", "cadeira", "mesa de jantar", "planta em vaso", "sofá" e "TV/monitor", de onde foram selecionadas duas classes para avaliar as diferentes MDPMs criadas. No critério de seleção foram selecionadas considerando as classes mais comuns nos diferentes conjuntos de dados de vídeo e trabalhos da literatura. Assim, as classes selecionadas são: carro, pessoa. Na Figura 4.2 pode-se observar imagens que contém pelo menos um objeto de cada uma das 3 classes selecionadas e seus respectivos bounding boxes (em amarelo)
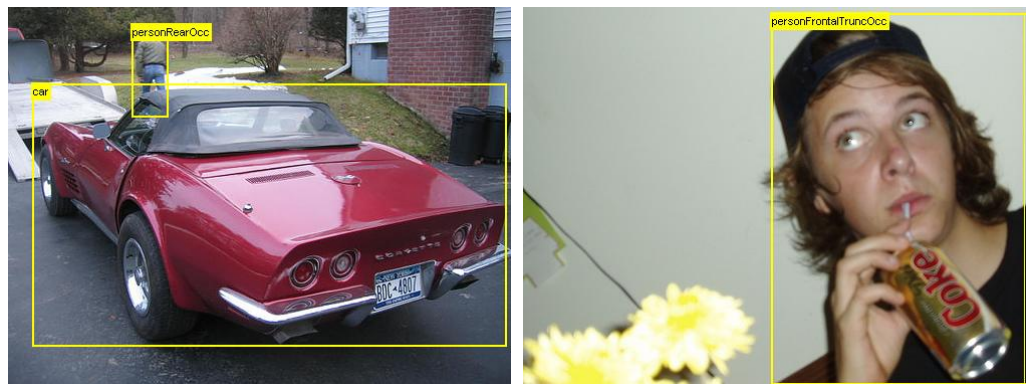

Figura 4.2: Imagens de exemplo das duas classes selecionadas. Reproduzidas de Everingham et al. (2010).

Para facilitar o uso nas principais tarefas de classificação, os conjuntos de dados são agrupa- 
dos em quatro conjuntos de imagens. Assim, tem-se os conjunto de dados de treinamento (1), o conjunto de validação (2), o conjunto resultante da união dos dado de treno e dos validação (3) e o conjunto de teste (4). Para cada imagem, o(s) objeto(s) da classe são anotados (delimitados) por um bounding box. A quantidade de imagens para cada um dos 4 grupos e para cada classe do conjunto de dados 2007 é mostrada na Tabela 4.1. A tabela resume o número de objetos e imagens (contendo pelo menos um objeto da classe dada) para classe. Os dados são particionados em 50\% para treinar/validar e $50 \%$ para testar (Everingham et al., a).

\begin{tabular}{l||rr|rr|rr|rr}
\hline \multirow{2}{*}{ CLASSE } & \multicolumn{2}{|c|}{ Treno (1) } & \multicolumn{2}{|c|}{ Validação (2) } & Treno-Validação (3) & \multicolumn{2}{|c}{ Teste (4) } \\
\cline { 2 - 9 } & Imagens & Objetos & Imagens & Objetos & Imagens & Objetos & Imagens & Objetos \\
\hline Carro & 376 & 625 & 337 & 625 & 713 & 1.250 & 721 & 1.201 \\
Pessoa & 1.025 & 2.358 & 983 & 2.332 & 2.008 & 4.690 & 2.007 & 4.528 \\
\hline TOTAL & 1.768 & 3.492 & 1.721 & 3.469 & 3.489 & 6.961 & 3.445 & 6.699 \\
\hline
\end{tabular}

Tabela 4.1: Resumo da quantidade de imagens e objetos etiquetadas como 'non-difficult', para duas classes, no conjunto de dados 2007 de Everingham et al. (a).

\subsection{Processo de deteç̧ão de objetos em vídeos}

Consiste em aplicar a MDPM de um objeto, obtida no passo anterior, em cada quadro de cada vídeo. A Figura 4.3 ilustra o processo, onde recebe como entrada o modelo (uma MDPM) e devolve os bounding boxes (detecções) para cada quadro.

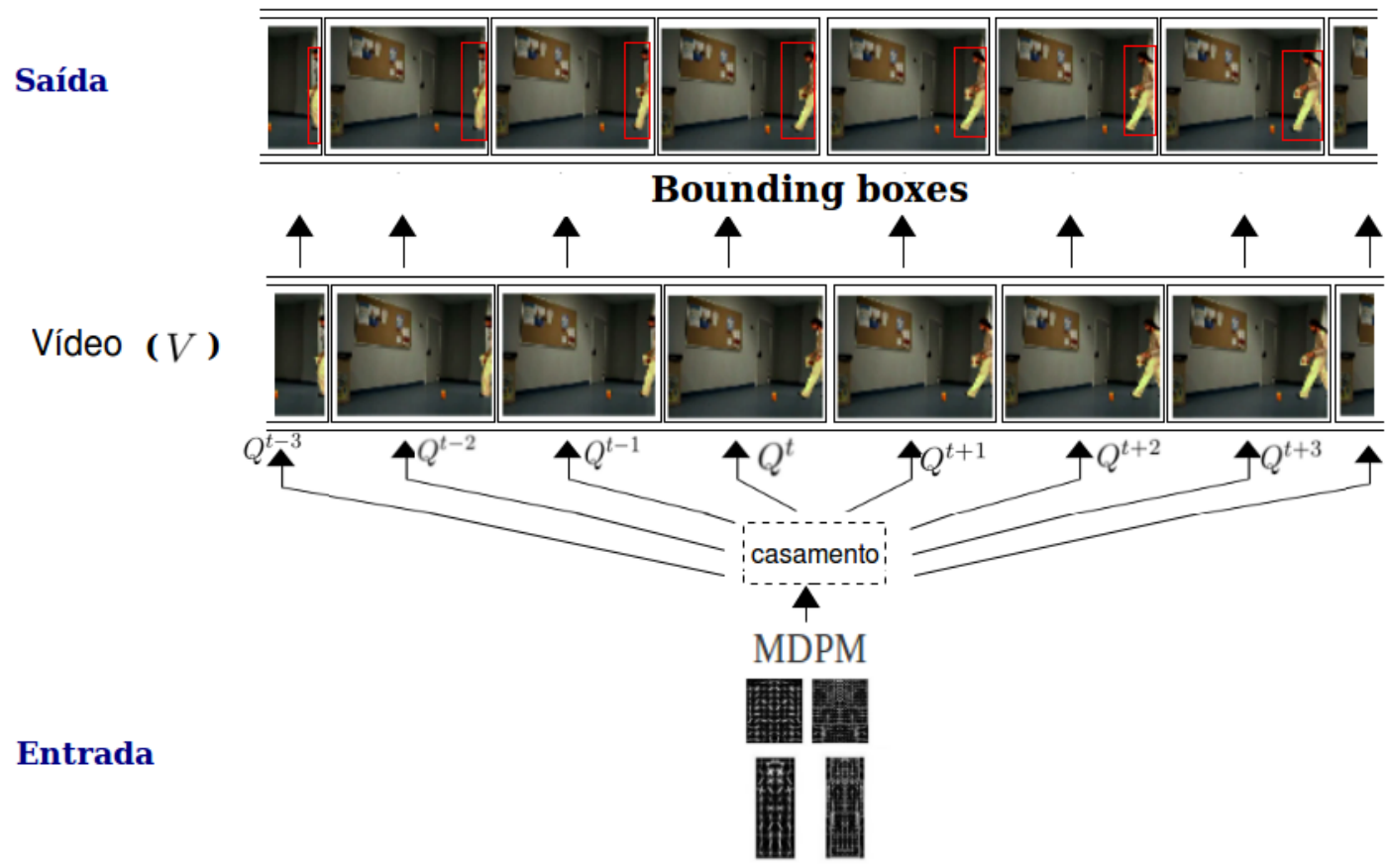

Figura 4.3: Processo de deteç̧ão num vídeo, usando a MDPM do objeto.

Por outro lado, quando tratamos com vídeos, podemos ter aqueles gerados por uma câmera estacionária ou por uma câmera dinâmica. Referimos como câmera estacionária quando a câmera encontra-se fixa, sem movimento, num determinado lugar e como dinâmica quando a câmera pode se movimentar, por exemplo, montada num veículo, carrinho de bebê, entre outros. A Figura 4.4a ilustra duas câmeras montadas num veículo, o qual vai gerar dois vídeos (uma por cada câmera). Logo, ao tratarmos com vídeos de câmera estacionária, temos um certo conhecimento do ambiente. 


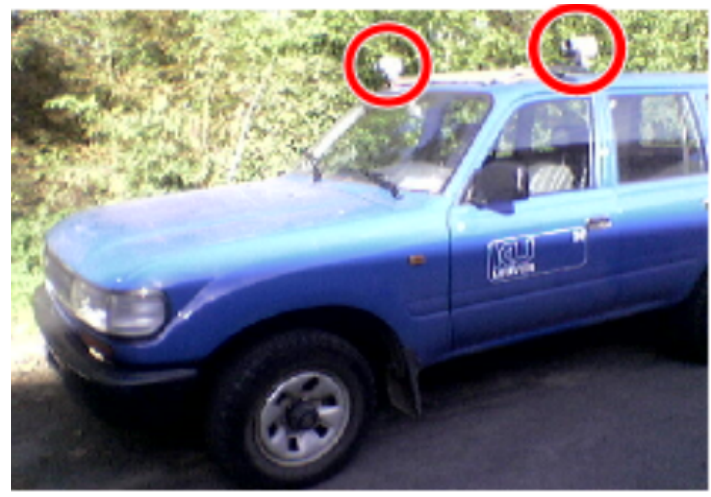

(a)

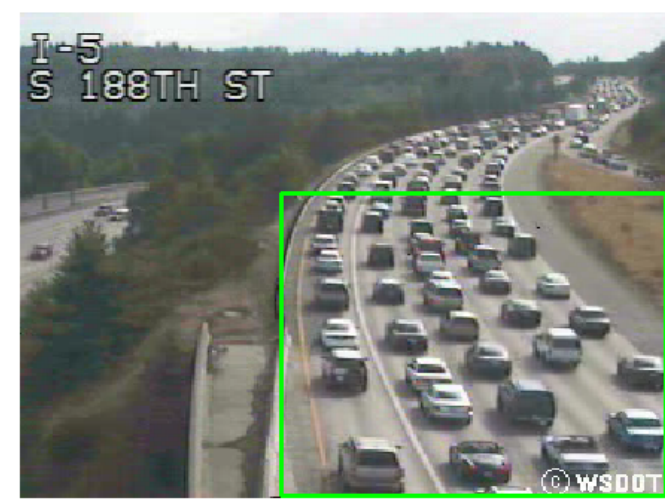

(b)

Figura 4.4: Exemplo de tipos de câmeras. (a) Câmeras montadas num veículo. Reproduzida de Leibe et al. (2008b). (b) Área de trânsito de carros conhecida.

Por exemplos na Figura 4.4b à região definida pelo retângulo verde é uma região onde usualmente tem-se carros com maior visibilidade a serem detectados.

Dado que, para este caso, os objetos normalmente estão em movimento, pode-se pensar que, com um simples estimador de movimento (Kamijo et al., 2001), captura-se os objetos de interesse. Adicionalmente, um extrator de fundo (Cheung e Kamath, 2004; Kamijo et al., 2001; Magee, 2004) poderia ser usado. Mas, e se um objeto aparece e logo fica estático? Por exemplo um carro estacionado no meio da rua (objeto estático), seguindo as técnicas mencionadas, ele não é detectado (Dalal e Triggs, 2005).

Agora, como tratar aos vídeos que foram obtidas por uma câmera dinâmica?. Dado que o fundo varia constantemente, então o modelo ou a definição do fundo é inviável. Adicionalmente, temos que os principais problemas para a tarefa de detecção considerando uma câmera dinâmica é o borramento, ou mudanças de iluminação mais rápidas, tal como se observa na Figura 4.5.
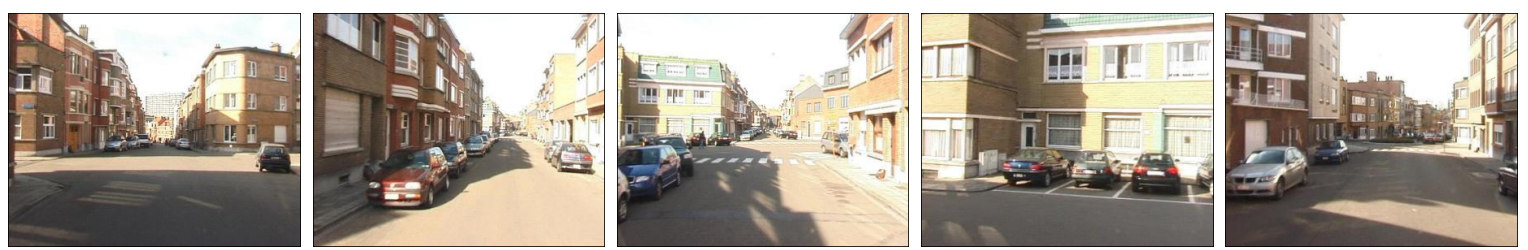

Figura 4.5: Imagens obtidas com uma câmera dinâmica. Extraídas de Leibe et al. (2007)

Em Leibe et al. (2006, 2008a,b), para estimar a geometria da cena, tais como parâmetros da câmera e o plano em 3D (ground plane) dos objetos, em cada passo do tempo foi usado Structurefrom-Motion (SfM) que eles definiram. Assim, eles auxiliam-se desses parâmetros no processo de deteç̧ão.

O processo usado neste trabalho para a detecção de objetos em vídeos obtidos tanto por uma câmera estacionária, como dinâmica, é definido no Algoritmo 4.1. O passo de decompor o vídeo em quadros, na prática, é realizado usando a função mmread.m ${ }^{1}$ do MATLAB. A estrutura dos vídeos obtida pela função é definida por:

- width: largura de cada quadro do vídeo.

- height: altura de cada quadro do vídeo.

- rate: taxa de quadro do vídeo. Caso não seja possível determina-lo, o valor será 1.

- nrFramesTotal: número total de quadros no vídeo.

\footnotetext{
${ }^{1}$ http://www.mathworks.com/matlabcentral/fileexchange/8028-mmread/content/mmread.m
} 


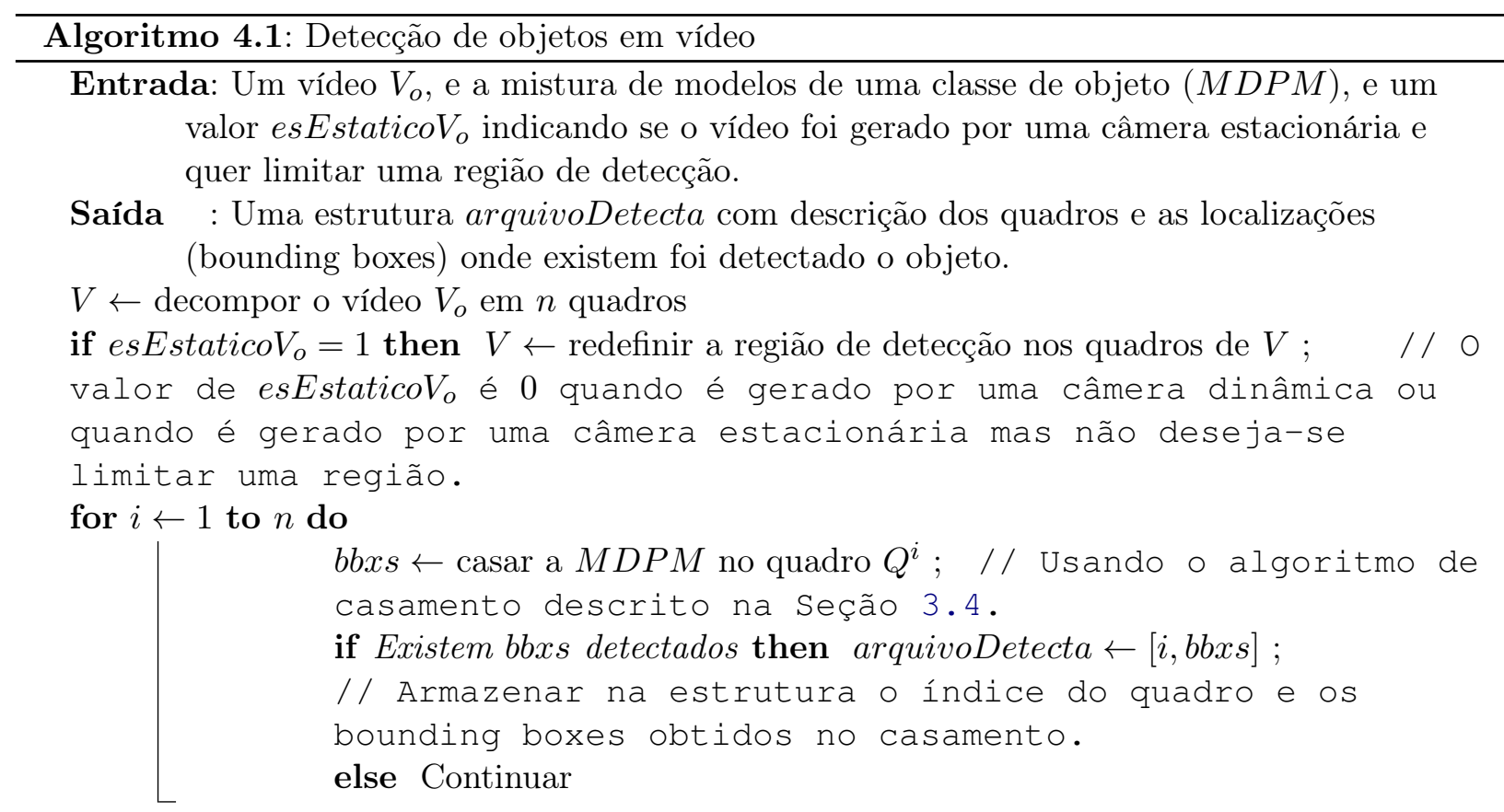

return arquivoDetecta;

- totalDuration: duração total do vídeo em segundos.

- frames: uma estrutura com os seguintes campos:

- cdata: matriz de [height $\mathrm{x}$ width x 3] contendo informação do quadro.

- colormap: vazio.

- times: o tempo do quadro em milissegundos.

Finalmente, foram realizados diferentes experimentos para duas classes definidas: carros e pessoas, cujos resultados são apresentados no seguinte capítulo. Os experimentos testam o método baseado em MDPM nos conjuntos de dados obtidos por uma câmera estacionária, ou dinâmica. Usou-se MDPMs simétricas e assimétricas. Em alguns casos, como não temos informação dos bounding boxes dos carros que aparecem nos vídeos, então o análise da performance é realizado visualmente. Em seguida descrevemos os conjuntos de dados de vídeo usados no trabalho, separados pela classe do objeto e o tipo da câmera usada para capturar eles.

\subsubsection{Vídeos para a classe carro com câmera estacionária}

\section{Conjunto 1}

O primeiro conjunto de dados é definido por Chan e Vasconcelos (2005), o qual é utilizado em tarefas de classificação do transito. Neste caso, o conjunto de dados é dividido em três classes: pesado (heavy), médio (medium) e leve (light). A classificação é feita baseada no tempo ou número de quadros que um carro em específico vai aparecer na sequência toda do vídeo. Adicionalmente, temos que os vídeos foram adquiridos em diferentes situações climáticas: em dias nublados, em dias ensolarados e em dias chuvosos onde em alguns casos a chuva cai na lente da câmera. A resolução dos quadros deste conjunto de dados é de 320 x 240 pixels. Para os experimentos foram selecionadas nove vídeos (veja a Tabela 4.2), que representam as três diferentes classes nas três diferentes situações mencionadas. 


\begin{tabular}{l|c|clc}
\hline Classe & ID & \multicolumn{1}{c}{ Nome do vídeo } & Clima & Quadros \\
\hline \multirow{3}{*}{ Leve } & V1 & $c c t v 052 x 2004080516 x 01640 . a v i$ & nublado & 48 \\
& V2 & $c c t v 052 x 2004080613 x 00015 . a v i$ & ensolarado & 53 \\
& V3 & $c c t v 052 x 2004080606 x 01821 . a v i$ & chuvoso & 47 \\
\hline \multirow{3}{*}{ Médio } & V4 & $c c t v 052 x 2004080516 x 01638 . a v i$ & nublado & 53 \\
& V5 & $c c t v 052 x 2004080516 x 01644 . a v i$ & ensolarado & 53 \\
& V6 & $c c t v 052 x 2004080618 x 00079 . a v i$ & chuvoso & 53 \\
\hline \multirow{3}{*}{ Pesado } & V7 & $c c t v 052 x 2004080516 x 01646 . a v i$ & nublado & 49 \\
& V8 & $c c t v 052 x 2004080517 x 01654 . a v i$ & ensolarado & 53 \\
& V9 & $c c t v 052 x 2004080618 x 00080 . a v i$ & chuvoso & 53 \\
\hline
\end{tabular}

Tabela 4.2: Sequencias de vídeo de transito de Chan e Vasconcelos (2005) utilizados nos experimentos.

\section{Conjunto 2}

O segundo conjunto de dados é formado pelo vídeo $A V S S_{-} P V_{-} E V A L . a v i$ de CAST e CPNI (2007). Neste caso, o vídeo foi criado para analisar os carros estacionados na rua, disponibilizando informação das zonas de trânsito, mas não vamos considerar essa informação nos experimentos. O vídeo tem 6586 quadros de resolução de 720 x 576 pixels. Na Figura 4.6, apresentam-se alguns exemplos de quadros deste vídeo, em específico mostra os quadros: 1, 2230, 4500, 5550.
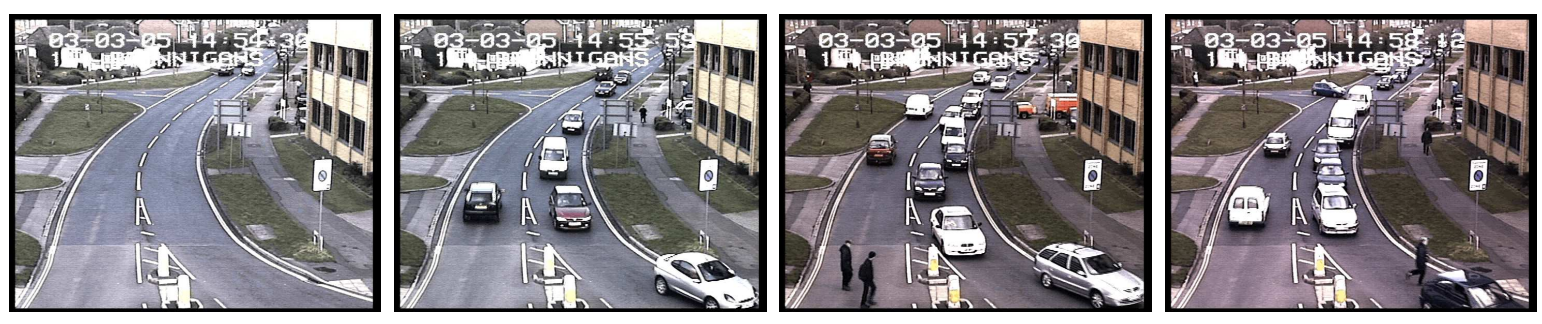

Figura 4.6: Alguns quadros do vídeo AVSS_PV_EVAL.

Como não temos os bounding boxes para este vídeo, então decidimos etiquetar algumas sequências dele. Dividimos o vídeo $A V S S_{-} P V_{-} E V A L$.avi CAST e CPNI (2007) em sequências de trinta segundos. Isto é, o vídeo todo tem 21000 quadros e dividimô-lo em 26 segmentos de 750 quadros e um segmento final de 1500 quadros. Selecionamos quatro das sequências, a primeira, a segunda, a sequência quinze e sequência vinte e seis e anotamos todos eles para testar.

Para anotar as sequências, elas precisaram ser convertidas ao formato FLV usando a ffmpeg (www.ffmpeg.org) sem perda da qualidade. Depois da seleção das sequências indicadas, tivemos que criar os bounding boxes para cada uma delas com o LabelMe Video Tool (Yuen et al., 2009) e fazer as anotações disponíveis no seguinte site: https://www.dropbox.com/sh/5nozkn3kbk1dtc9/ RjVqAt_0sH. Adicionalmente foi disponibilizado as funções para ler os arquivos XLM gerados pelo LabelMe Tool e para converter os arquivos ao formato PASCAL.

\subsubsection{Vídeos para a classe carro com câmera dinâmica}

Foi usado o vídeo Leuven de Leibe et al. (2007), que foi capturado por Nico and Kurt Cornelis e encontra-se disponível para propósitos não comerciais. Leuven contém 1175 pares de imagens (esquerda e direita) adquiridas com duas câmeras estéreo montadas em um veiculo em andamento 
e gravando a uma distância de aproximadamente 500 metros. Cada quadro tem uma resolução de 380 x 288 pixels e a taxa de quadros é de $25 f$ ps.

Para os experimentos foram selecionados os dados gerados pela câmera esquerda. Na Figura 4.7 mostram-se alguns exemplos de quadros do conjunto de dados. Este conjunto contém informação na calibração externa da câmera, o plano 3D estimado para cada quadro, e os bounding boxes dos carros presentes em cada quadro. Em alguns casos, tem-se quadros com carros que não contém seus respectivos bounding boxes, isso porque só encontram-se disponíveis os bounding boxes dos carros que encontram-se até uma distancia de 50 metros da câmera e visível pelo menos em 40-50\%. Assim, esta sequência contém 77 carros estáticos suficientemente visíveis e 4 carros em movimento.
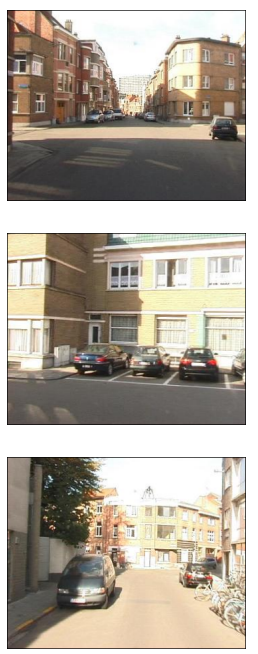
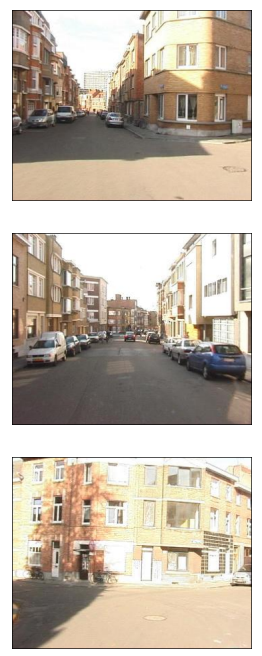

Figura 4.7: $A$
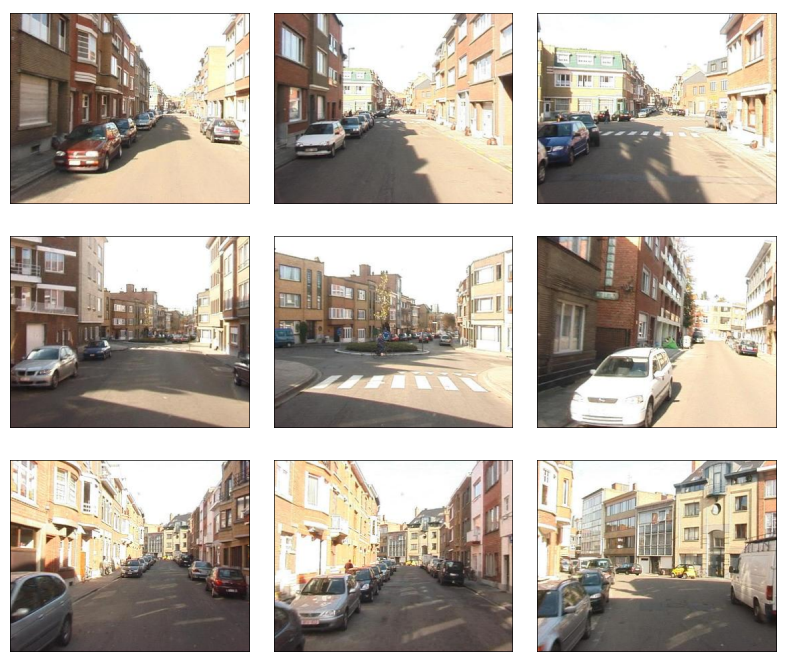
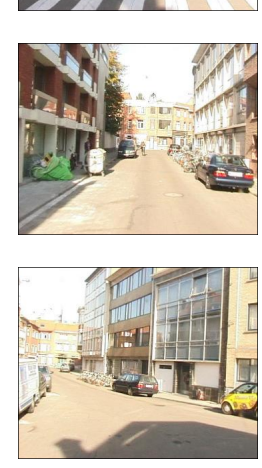

\subsubsection{Vídeos para a classe pessoa}

Foi usado o conjunto de dados Person Detection Dataset (PDds) de García-Martín et al. (2012) e que está disponibilizado no link http://www-vpu.eps.uam.es/PDds/index.html para propósitos acadêmicos. Os vídeos incluem objetos rígidos e não rígidos e consideram cenas interiores e exteriores e diferentes complexidades de objetos e fundo. Os objetos também encontram-se em trajetórias com interseção ou não, com oclusões e em diferentes contextos.

Os autores do PDds, classificaram os vídeos em diferentes categorias que representam a complexidade dos mesmos. As categorias são baseadas nas dificuldades de alguns fatores que podem afetar o comportamento do algoritmo de detecção. Os fatores têm relação com as pessoas. Por exemplo: se elas estão em movimento ou paradas numa cena. Os fatores também têm relação com o fundo da cena. Desta forma, temos que a dificuldade de um fator é definida (em García-Martín et al. (2012)): pelo número de pessoas numa cena, pelas velocidades com as quais elas aparecem, pelas oclusões a variações de pose, pelas variações de roupas, ou objetos que carregam. Adicionalmente, é definida pela dificuldade de extrair os objetos (pessoas) do fundo, pelas diferentes texturas presentes, pelas mudanças de iluminação, reflexões, sombras e objetos que pertencem ao fundo. A Tabela 4.3 apresenta uma descrição desses fatores em cada uma das categorias que foram definidas em García-Martín et al. (2012).

Os vídeos pertencem a diferentes conjuntos de dados públicos, como VISOR ${ }^{2}$ e hall monitor ${ }^{3}$, que são usados em tarefas de deteç̧ão/classificação de objetos. Os vídeos tem resolução de: $352 \times 288$ $(C V S G), 320 \times 256($ VISOR) e de $352 \times 240$ (hall monitor). A Tabela 4.4 apresenta os vídeos que foram usadas nos experimentos.

Considerando os fatores definidos, temos que, com relação às pessoas, alguns vídeos contém a mesma ação e o que os diferença é o fundo da cena por categoria. Isto significa que alguns

\footnotetext{
${ }^{2}$ http://imagelab.ing.unimore.it/visor/index.asp

${ }^{3}$ http://trace.eas.asu.edu/yuv/
} 


\begin{tabular}{|c|c|c|c|}
\hline Categoria & Fator & Complexidade & Descrição \\
\hline \multirow[t]{2}{*}{ C 1} & Pessoa & Baixa & $\begin{array}{l}\text { Mudança limitados de pose, variações de tamanho, interações, } \\
\text { etc. Sem oclusões. }\end{array}$ \\
\hline & Fundo & Baixa & $\begin{array}{l}\text { Fundos com pouca textura. Fundo estático. Sem mudanças de } \\
\text { iluminação, reflexões, sombras, etc. }\end{array}$ \\
\hline \multirow[t]{2}{*}{ C 2} & Pessoa & Média & $\begin{array}{l}\text { Mudança de pose, variações de tamanho, interações, etc. Sem } \\
\text { oclusões. }\end{array}$ \\
\hline & Fundo & Baixa & $\begin{array}{l}\text { Fundos com pouca textura. Fundo estático. Sem mudanças de } \\
\text { iluminação, reflexões, sombras, etc. }\end{array}$ \\
\hline \multirow[t]{2}{*}{ C 3} & Pessoa & Média & $\begin{array}{l}\text { Mudança de pose, variações de tamanho, interações, etc. Oclu- } \\
\text { sões parciais. }\end{array}$ \\
\hline & Fundo & Média & $\begin{array}{l}\text { Fundos com algo de textura. Mudança de iluminação, refle- } \\
\text { xões, sombras, etc. }\end{array}$ \\
\hline \multirow[t]{2}{*}{ C 4} & & Alta & $\begin{array}{l}\text { Múltiplos mudanças de pose, variações de tamanho, intera- } \\
\text { ções, etc. Numerosas oclusões parciais. }\end{array}$ \\
\hline & Fundo & Baixa & $\begin{array}{l}\text { Fundos com pouca textura. Fundo estático. Sem mudanças de } \\
\text { iluminação, reflexões, sombras, etc. }\end{array}$ \\
\hline \multirow[t]{2}{*}{ C 5} & Pessoa & Alta & $\begin{array}{l}\text { Múltiplos mudanças de pose, variações de tamanho, intera- } \\
\text { ções, etc. Numerosas oclusões parciais. }\end{array}$ \\
\hline & Fundo & Alta & $\begin{array}{l}\text { Fundos com muita textura. Fundo multimodal, mudanças de } \\
\text { iluminação, reflexões, sombras, etc. }\end{array}$ \\
\hline
\end{tabular}

Tabela 4.3: Categorias definidas nos vídeos do PDds.

\begin{tabular}{c||clc|clc}
\hline Categoria & ID & Nome do vídeo & Quadros & ID & Nome do vídeo & Quadros \\
\hline \multirow{2}{*}{ C 1} & $V 11$ & $C V S G_{-} S 1 . a v i$ & 229 & $V 13$ & $C V S G_{-} S 5 . a v i$ & 166 \\
& $V 12$ & $C V S G_{-} S 4 . a v i$ & 283 & $V 14$ & $C V S G_{-} S 16 . a v i$ & 619 \\
\hline \multirow{3}{*}{ C 2} & $V 21$ & $C V S G_{-} S 2 . a v i$ & 427 & $V 24$ & $V I S O R_{-} S 2 . a v i$ & 190 \\
& $V 22$ & $C V S G_{-} S 3 . a v i$ & 334 & $V 25$ & $V I S O R_{-} S 5 . a v i$ & 534 \\
& $V 23$ & $C V S G_{-} S 12 . a v i$ & 367 & & & \\
\hline $\mathrm{C} \mathrm{3}$ & $\mid V 31$ & $C V S G_{-} S 13 . a v i$ & 367 & $V 32$ & $h a l l_{-}$monitor.avi & 300 \\
\hline \multirow{3}{*}{$\mathrm{C} 4$} & $V 41$ & $C V S G_{-} S 6 . a v i$ & 589 & $V 44$ & $C V S G_{-} S 14 . a v i$ & 794 \\
& $V 42$ & $C V S G_{-} S 7 . a v i$ & 1513 & $V 45$ & $C V S G_{-} S 17 . a v i$ & 1378 \\
& $V 43$ & $C V S G_{-} S 8 . a v i$ & 1027 & & & \\
\hline \multirow{3}{*}{$\mathrm{C} \mathrm{5}$} & $V 51$ & $C V S G_{-} S 9 . a v i$ & 1513 & $V 54$ & $C V S G_{-} S 15 . a v i$ & 794 \\
& $V 52$ & $C V S G_{-} S 10 . a v i$ & 1027 & $V 55$ & $C V S G_{-} S 18 . a v i$ & 1378 \\
& $V 53$ & $C V S G_{-} S 11 . a v i$ & 1513 & & & \\
\hline
\end{tabular}

Tabela 4.4: Sequencias de vídeo do PDds usados nos experimentos.

vídeos foram obtidos de forma sintética (todas as linhas da primeira coluna na Figura 4.8) e outros representam o mundo real (segunda e terceira linha da segunda coluna na Figura 4.8). Por exemplo, o vídeo $V 23$ é igual na ação ao vídeo $V 31$, ambos tem a seguinte ação: primeiro entra um homem na cena com diversas poses e em seguida entra uma mulher correndo e em diferentes poses e logo saem da cena, mas a principal diferença é o fundo que depende da categoria (desta forma ambos são vídeos sintéticos). Adicionalmente, os vídeos $V 42, V 51$ e $V 53$ são iguais em ação, onde inicialmente entra um homem na cena deixa um objeto no chão depois de um tempo entra outro na cena, vira-se e deixa outro objeto no chão e sai, e finalmente entra um outro homem que fica sentado no chão e 
sai da cena. Também, temos que os vídeos $V 43$ com $V 52, V 44$ com $V 54$, entre outros.

$\mathrm{Na}$ Figura 4.8 ilustra-se exemplos de 2 vídeos por categoria, onde todos representam ações diferentes. Na categoria $C 1$ as primeiras 3 colunas pertencem ao vídeo $V 12$ e as 3 últimas colunas ao vídeo $V 14$. Na categoria $C 2$ os quadros pertencem aos vídeos $V 21$ e $V 25$ respectivamente cada 3 colunas. Na categoria $C 3$ as primeiras 3 colunas pertencem ao vídeo $V 31$ e as 3 últimas colunas ao vídeo $V 32$. Na categoria $C 4$ os quadros pertencem aos vídeos $V 43$ e $V 45$ respectivamente cada 3 colunas.
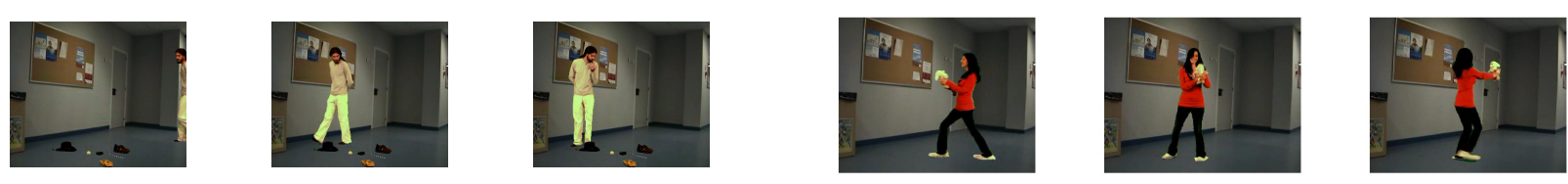

(a) Categoria 1
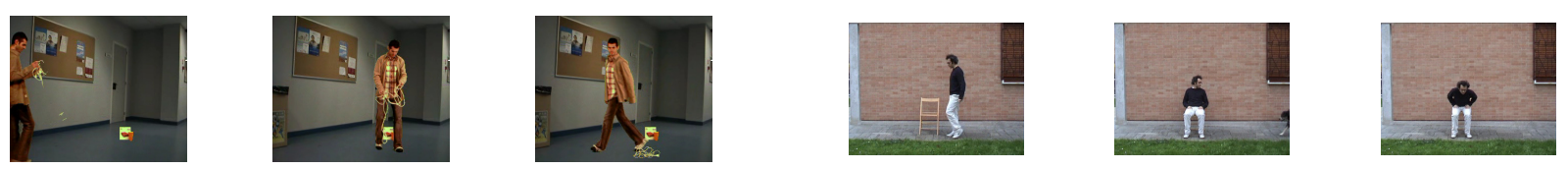

(b) Categoria 2
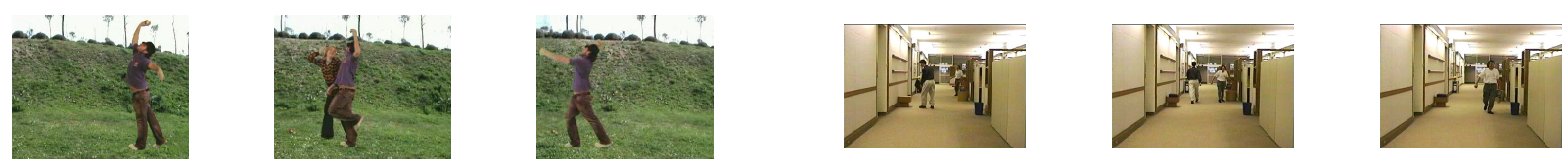

(c) Categoria 3
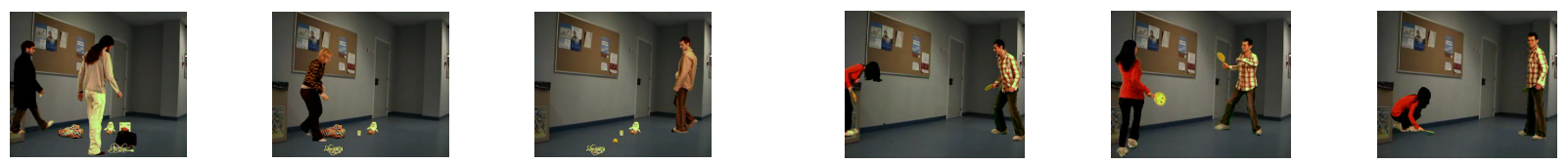

(d) Categoria 4

Figura 4.8: Exemplos de dois diferentes vídeos de 4 categorias do PDds. 


\section{Experimentos e resultados}

Neste capítulo descrevemos os experimentos realizados (Seção 5.2) e os resultados obtidos para cada um dos experimentos definidos, considerando as métricas definidas na Seção 5.1. Todos os processos usados foram implementados e executados em MATLAB R2009b e C ++ no sistema operacional GNU/Linux (Foundation), num computador Intel(R) Xeon(R) CPU de 2.6GHz e $6 \mathrm{CPU}$ núcleos e num Intel(R) Core(TM) i7 CPU de $2.6 \mathrm{GHz}$ e 4 CPU cores.

\subsection{Métricas}

Para cada imagem/quadro, aplica-se o algoritmo de detecção de objetos e, como resposta, obtemos os bounding boxes preditos e um valor de confidência para a predição. Seguindo as regras definidas no desafio PASCAL VOC, uma detecção é considerada correta ou verdadeiro positivo $(V P)$ quando a área de sobreposição $\left(a_{s}\right)$ entre o bounding box predito $\left(B_{p}\right)$ com o bounding box anotado $\left(B_{g t}\right)$ no conjunto de dados, é maior que $50 \%$, caso contrário é um falso positivo. A área $a_{s}$ é definida como:

$$
a_{s}=\frac{\operatorname{area}\left(B_{p} \cap B_{g t}\right)}{\operatorname{area}\left(B_{p} \cup B_{g t}\right)}
$$

Adicionalmente, verdadeiros positivos tornam-se falsos positivos $(F P)$ caso sobreponham várias detecções com o bounding box anotado. Neste caso, apenas um deles, aquele com maior valor de confiança, é considerado um verdadeiro positivo e os outros serão considerados como falsos positivos. O falso negativo $(F N)$ representa aquele bounding box que está presente no conjunto de teste mas que não foi predito pelo algoritmo.

As métricas definidas para avaliar os nossos resultados, descritas a seguir, são baseados nas regras do desafio PASCAL VOC, em precisão (precision), revocação(recall), na média de precisão (Average Precision - AP), e na curva de precision-recall. Adicionalmente, foi considerado o falso positivo por imagem (False Positive Per Image - FPPI), a curva de taxa de perda (Miss Rate) vs. FPPI e a pontuação F. Ao longo do texto, usaremos os termos em inglês mesmo para as métricas. O objetivo é ter um alto valor de recall e precision, isto é, valores próximos de 1 numa faixa de $[0,1]$.

- A precision é dada pela fração dos bounding boxes preditos que são detecções corretas, ou verdadeiros positivos, definida por:

$$
\text { precision }=\frac{V P}{V P+F P} \text {. }
$$

- A recall é a fração das deteç̧ões obtidas, definida:

$$
\text { recall }=\frac{V P}{V P+F N} .
$$


- O average precision (AP) é obtido com a Equação (5.4), onde $p r_{\text {interp }}(\tilde{r})$ é uma interpolação dos valores que têm a precision máxima em todas as revocações $\tilde{r}: \tilde{r} \geq r$.

$$
\operatorname{AP}=\frac{1}{11} \sum_{r \in 0,1, .2, \ldots, 1}\left[\max _{\tilde{r}: \tilde{r} \geq r} \operatorname{pr}_{\text {interp }}(\tilde{r})\right]
$$

- Os falsos positivos por imagens (FPPI) é obtido por:

$$
\operatorname{FPPI}=\frac{F P}{\# \text { total de quadros }}
$$

- A Miss Rate $(1-r e c a l 1)$ e a curva Miss Rate vs. FPPI.

- A pontuação $F$, que pode ser interpretada como uma média ponderada da precision e recall, é definida por:

$$
F=2 * \frac{\text { precision } * \text { recall }}{\text { precision }+ \text { recall }}
$$

\subsection{Descrição dos experimentos}

Em seguida, a lista dos experimentos realizados:

E1: Definição dos parâmetros das MDPMs (Seção 5.3). Como foi descrito uma MDPM é definida por $m$ componentes e cada componente por um modelo de $n$ partes, surgindo assim a pergunta de "Qual é o melhor valor para $m$ e $n$ ?". Desta forma, definimos como parâmetros importantes das MDPMs o número de componentes da mistura e o número de partes do modelo. Por isso, vamos encontrar valores para os parâmetros de uma MDPM com os quais obtenham-se melhores resultados. Usaremos o conjunto de imagens do PASCAL VOC 2007, para criar diferentes MDPMs simétricas e assimétricas para as classes carro e pessoa. Aplicaremos elas ao conjunto de teste do PASCAL VOC 2007 e faremos uma comparação com os resultados obtidos por outros métodos em o mesmo conjunto de teste.

Baseados nos resultados obtidos para imagens, selecionam-se algumas MDPMs e aplica-se cada uma em diferentes vídeos: obtidos por uma câmera estacionária, ou uma câmera dinâmica, para a detecção de objetos pertences às classes carro e pessoa separadamente. Em todos os conjuntos de vídeos têm-se objetos em movimento e, adicionalmente, objetos estáticos ou estacionários. Estes experimentos são descritos a seguir.

E2: Deteç̧ão de carros em vídeos obtidos por uma câmera estacionária sem ter informação dos bounding boxes(Subseção 5.4.1). Pelo fato de não ter informação dos bounding boxes a análise é feita de maneira visual. Para este conjunto de vídeos, é definida uma região de detecção tal como foi mencionado no capítulo anterior. Foram usadas MDPMs de três componentes da mistura e seis, oito e dez partes do modelo.

E3: Deteç̧ão de carros em vídeos obtidos por uma câmera estacionária tendo informação dos bounding boxes (Subseção 5.4.2). Na verdade, não encontram-se disponíveis os bounding boxes do vídeo, então decidimos etiquetar algumas sequências dele tal como foi mencionado no capítulo anterior.

Testamos usando MDPMs de dois e três componentes da mistura e seis, oito e dez partes do modelo. Adicionalmente, comparamos os nossos resultados obtidos com dois modelos de carro criados por Geiger et al. (2011) e que estão disponíveis através do KITTI Vision Benchmark Suite (Geiger et al., to appear). Definimos como model1, o modelo chamado como car_0_2012_10_19-14_40_47_model e como model2, o modelo car_2_2012_10_0420_13_57_model. Os modelos tem 16 componentes e 8 partes do modelo, só que eles foram 
treinados usando quadros de vídeos obtidos por uma câmera dinâmica. Os limiares dos modelos são de 0.6330 e 0.5778 para model 1 e model 2 , respectivamente. Desta forma, vamos poder verificar como que se comportam os modelos treinados com imagens específicas.

E4: Detecção de carros em vídeos obtidos por uma câmera dinâmica (Seção 5.5). Neste caso, encontra-se disponível informação do ground plane, mas ela não é considerada neste trabalho. De maneira similar que ao experimento anterior, testamos usando MDPMs de dois e três componentes da mistura e seis, oito e dez partes do modelo. Adicionalmente, comparamos os nossos resultados com os obtidos usando os modelos criados model1 e model2 por Geiger et al. (2011).

E5: Detecção de pessoas em vídeos (Seção 5.6). Contém vídeos captados por câmeras estacionárias. Para este conjunto de vídeos, não é definida uma região de deteç̧ão dado que os objetos apresentam-se na maior parte do quadro. Foram usadas MDPMs de três componentes da mistura e seis, oito e dez partes do modelo.

Cabe mencionar que para a realização dos experimentos foi usado o código disponível pelos autores de Felzenszwalb et al. (2010b) e de Felzenszwalb et al. em http://www.cs.brown.edu/ pff/ latent-release3/ e em http://www.cs.brown.edu/ pff/latent-release4/, respectivamente.

\subsection{Parâmetros das MDPMs}

Primeiramente, foram criadas (treinadas) diferentes MDPMs variando o número de componentes da mistura e o número de partes do modelo. O número de componentes pode ser um elemento de $\{1,2,3,4\}$ e o número de partes para todas as classes é um elemento de $\{4,6,8,10\}$. As MDPMs obtidas são denominadas como $1 M 4 P, 1 M 6 P, 1 M 8 P, 1 M 10 P, 2 M 4 P, 2 M 6 P, 2 M 8 P, 2 M 10 P$, $3 M 4 P, 3 M 6 P, 3 M 8 P, 3 M 10 P, 4 M 4 P, 4 M 6 P, 4 M 8 P, 4 M 10 P$. Para a classe pessoa, adicionalmente, quando se usam 3 componentes de mistura, o número de partes pode também ser um elemento de $\{5,7,9\}$.

Um exemplo de MDPM assimétrica obtidas para a classe pessoa é mostrada na Figura 5.1. O exemplo apresentado é de uma MDPM de 2 componentes da mistura e 8 partes do modelo, lembrando que para as MDPMs assimétricas estamos considerando a componente de vista esquerda com a da direita como uma só componente. Na figura a primeira coluna mostra o filtro raiz, a segunda coluna mostra os filtros parte e a terceira coluna mostra o modelo espacial para cada parte. Da figura, pode-se observar a filtros parte para cabeça, tronco, pernas, entre outros.

Em seguida, para verificar como se comportam cada uma das MDPMs criadas num processo de deteç̧ão, foram feitos experimentos de deteç̧ão de objetos de uma determinada classe nas imagens de teste de PASCAL VOC 2007 e comparados com alguns métodos da literatura. A saída do processo é um bounding box, o qual deve coincidir com os anotados no conjunto de dados. Na prática, o sistema devolve um conjunto de bounding boxes com suas pontuações correspondentes e muitos deles podem estar superpostos.

O tempo de treinamento por classe (em aproximado 1000 imagens) foi de aproximadamente 4 horas e para testar (em aproximado 1000 imagens) de aproximadamente 2 horas. Atualmente é possível reduzir esse tempo considerando uma outra implementação, como aquela disponível em http://libccv.org/doc/doc-dpm/.

Todos os resultados obtidos neste experimento são apresentados a seguir.

\section{Resultados}

Os resultados de average precision obtidos ao aplicar as diferentes MDPMs treinadas ao conjunto de teste do PASCAL VOC 2007 para a classe carro e pessoa, são apresentados na Tabela 5.1. Na tabela, o melhor resultado de AP para cada classe, tanto para MDPMs simétricas como assimétricas, esta diferenciada em azul e o segundo melhor resultado em negrito. 

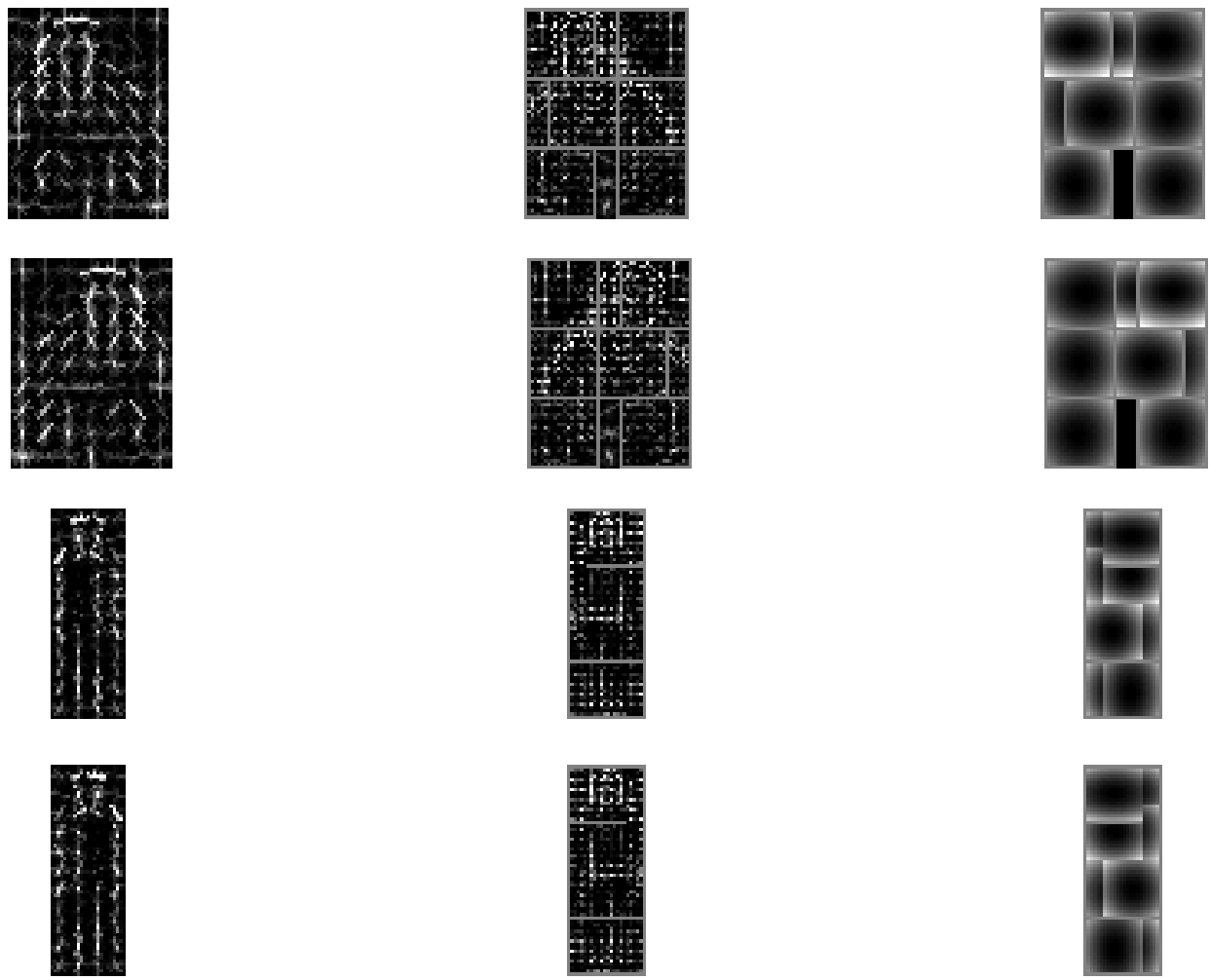

Figura 5.1: $M D P M$ assimétrica de 2 componentes e 8 partes para a classe pessoa.

\begin{tabular}{l|cc|l|cc}
\hline & \multicolumn{5}{c}{ CARRO } \\
MDPM's & Simétrica & Assimétrica & MDPM's & Simétrica & Assimétrica \\
\hline 1M 4P & - & 0.517 & 3M 4P & 0.468 & 0.572 \\
1M 6P & - & 0.544 & 3M 6P & 0.486 & $\mathbf{0 . 5 7 9}$ \\
1M 8P & - & 0.545 & 3M 8P & 0.503 & $\mathbf{0 . 5 8 3}$ \\
1M 10P & - & 0.543 & 3M 10P & $\mathbf{0 . 5 1 1}$ & 0.568 \\
2M 4P & 0.482 & 0.546 & 4M 4P & 0.486 & 0.571 \\
2M 6P & 0.502 & 0.568 & 4M 6P & 0.485 & - \\
2M 8P & 0.495 & 0.564 & 4M 8P & 0.497 & - \\
2M 10P & 0.488 & 0.559 & 4M 10P & $\mathbf{0 . 5 0 5}$ & - \\
\hline & & \multicolumn{2}{c}{ PESSOA } & & \\
& Simétrica & Assimétrica & MDPM's & Simétrica & Assimétrica \\
\hline 1M 4P & - & 0.352 & 3M 6P & $\mathbf{0 . 3 7 9}$ & 0.418 \\
1M 6P & - & 0.345 & 3M 7P & 0.374 & 0.418 \\
1M 8P & - & 0.340 & 3M 8P & 0.367 & $\mathbf{0 . 4 2 5}$ \\
1M 10P & - & 0.334 & 3M 9P & $\mathbf{0 . 3 7 6}$ & $\mathbf{0 . 4 2 1}$ \\
2M 4P & 0.349 & 0.387 & 3M 10P & 0.344 & 0.420 \\
2M 6P & 0.367 & 0.402 & 4M 4P & 0.338 & 0.401 \\
2M 8P & 0.368 & 0.401 & 4M 6P & 0.357 & - \\
2M 10P & 0.373 & 0.406 & 4M 8P & 0.354 & - \\
3M 4P & 0.355 & 0.414 & 4M 10P & 0.352 & - \\
3M 5P & 0.354 & 0.405 & & & \\
\hline
\end{tabular}

Tabela 5.1: AP obtidos para a classe carro e pessoa, mudando os parâmetros das MDPMs simétricas e assimétricas.

Dos resultados obtidos para a classe carro, observamos que, para os APs, temos o melhor valor com 0.511 para uma MDPM simétrica de 3 componentes e 10 partes, e para uma MDPM assimétrica 
tem-se um AP de 0.583 com uma MDPM de 3 misturas e 8 partes. Desta forma temos que os melhores resultados estão composto regularmente por MDPM de 3 componentes e de 10 ou 8 partes. Se analisamos com respeito ao AP, vemos que o melhor resultado foi obtido pela MDPM assimétrica. Provavelmente porque ela captura melhor as vistas laterais dos carros. A segunda linha na Figura 5.2 ilustra a curva de precision vs recall e o AP para cada MDPM de 2 e 3 componentes.

Dos resultados obtidos para a classe Pessoa, observamos que para os APs, temos o melhor valor com 0.379 para uma MDPM simétrica de 3 componentes e 6 partes, e para uma MDPM assimétrica têm-se um AP de 0.425 com uma MDPM de 3 componentes e 8 partes. Destes experimentos, têmse que os melhores resultados estão composto regularmente por MDPM de 3 componentes e 6 e 8 partes. Se analisamos com respeito ao AP, vemos que o melhor resultado foi obtido pela MDPM assimétrica, porque ela captura melhor as vistas de lado. A última linha na Figura 5.2 ilustra a curva de precision vs recall e o AP para cada MDPM de 2 e 3 componentes.

Em geral, observa-se das curvas precision vs. recall que as MDPMs mostram um melhor desempenho para poucas componentes da mistura, como 3 componentes. Adicionalmente, mostram melhores resultados para MDPMs assimétricas, dado que representam de forma separada o linha de visada do lado esquerdo e direito de um objeto. O fato de ter melhores resultados sem muitas componentes pode ser, basicamente, porque como os exemplos de treinamento são atribuídos para uma única componente, a quantidade de exemplos de treinamento por componente decrementa linearmente com o número de componentes, o qual implica uma generalização fraca. O aumento do número de componentes e o número de partes de um modelo implica também um aumento no custo computacional, tanto no treinamento, como no teste.

\section{Comparação com outros métodos}

Foi feita a comparação dos melhores resultados obtidos usando o conjunto de dados de teste do PASCAL VOC 2007, tanto para MDPMs simétricas como assimétricas, com algumas das diferentes técnicas presentes na literatura como: Chen et al. (2010); Mottaghi (2012); Song et al. (2011); Vedaldi et al. (2009). Essa comparação é apresentada na Tabela 5.2, com o melhor valor obtido em vermelho, e onde observa-se que a técnica utilizada supera às outras nesse tipo de dado.

Para a classe carro, das MDPMs criadas selecionamos: uma MDPM simétrica de 3M 10P e uma MDPM assimétrica de 3M 8P com as quais foram obtidos os melhores resultados anteriormente. Comparando essas duas MDPMs com a literatura, temos que o melhor resultado foi obtido considerando a MDPM assimétrica. Para a classe pessoa foram selecionadas: uma MDPM simétrica de 3M 6P e uma MDPM assimétrica de 3M 8P. Observa-se que os melhores resultados foram obtidos considerando-se três componentes. Isso pode ser devido à variabilidade das poses e representação da classe pessoa nas imagens de treinamento e teste. Finalmente, de forma similar que para a classe carro, temos que o melhor resultado foi obtido considerando a MDPM assimétrica.

\begin{tabular}{l|cc}
\hline MÉTODO & CARRO & PESSOA \\
\hline MDPM Simétrica & $0.511(3 \mathrm{M} \mathrm{10P)}$ & $0.379(3 \mathrm{M} 6 \mathrm{P})$ \\
\hline MDPM Assimétrica & $\mathbf{0 . 5 8 3}(3 \mathrm{M} 8 \mathrm{P})$ & $\mathbf{0 . 4 2 5}(3 \mathrm{M} \mathrm{8P})$ \\
\hline MKL (Vedaldi et al., 2009) & 0.506 & 0.233 \\
\hline Active Masks Hierarchies (Chen et al., 2010) & 0.540 & 0.349 \\
\hline NUS-Context (Song et al., 2011) & 0.560 & 0.412 \\
\hline Superposition potential (SP) (Mottaghi, 2012) & 0.539 & 0.265 \\
\hline SP and HOG-bundle potentials (Mottaghi, 2012) & 0.529 & 0.387 \\
\hline
\end{tabular}

Tabela 5.2: Comparação dos melhores AP obtidos usando o conjunto de dados de teste do PASCAL VOC $200 \%$. 

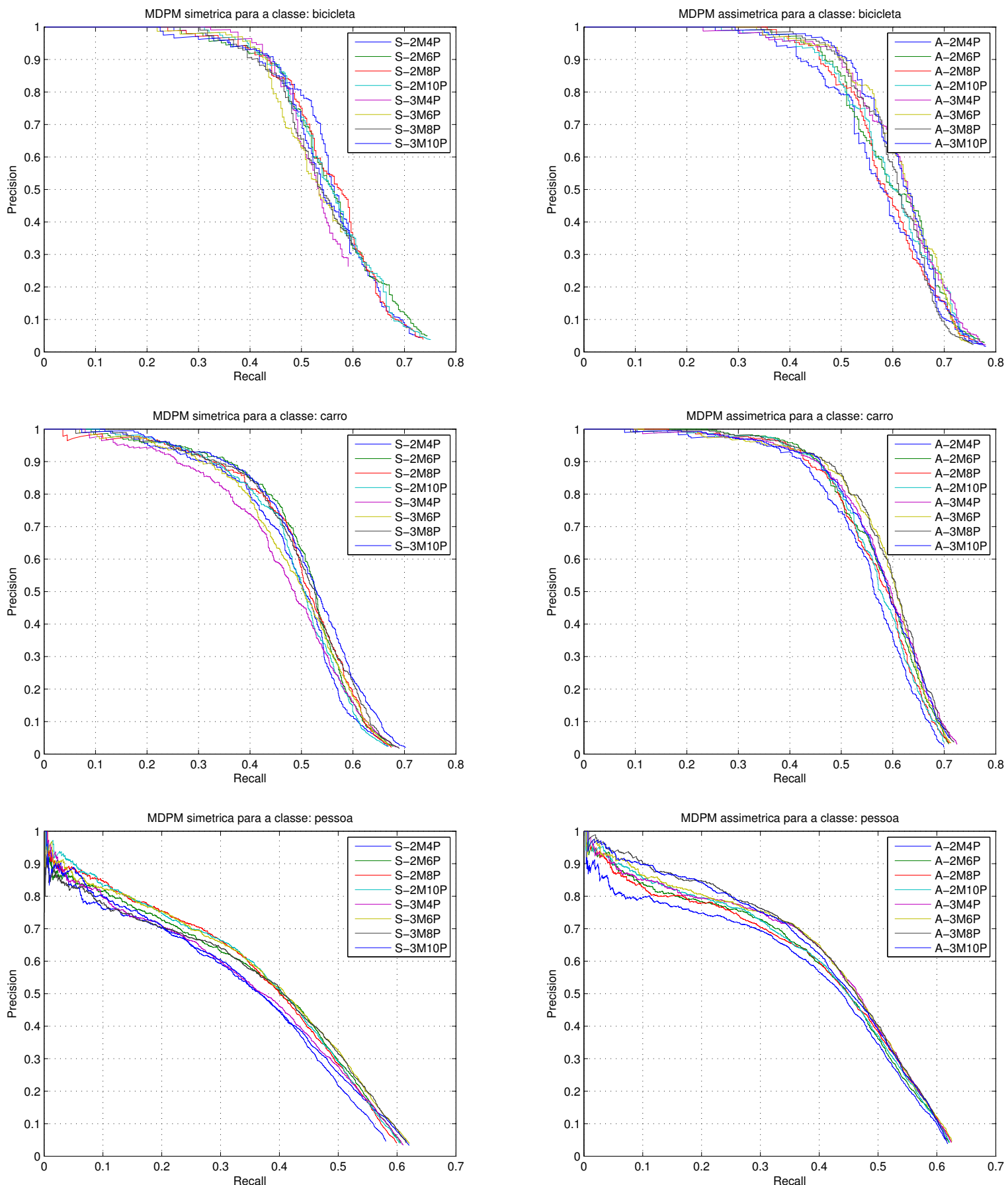

Figura 5.2: Curva precision vs recall para MDPMs simétricas (primeira coluna) e assimétricas (segunda coluna) de 2 e 3 componentes para a classe bicicleta (primeira fila), carro (segunda fila) e pessoa (última fila).

Alguns exemplos de detecção obtidos usando uma MDPM de 3 componentes e 6 partes são mostrados na Figura 5.3. Na figura o bounding box vermelho representa ao filtro raiz e os bounding boxes em azul aos filtros parte do objeto detectado. 


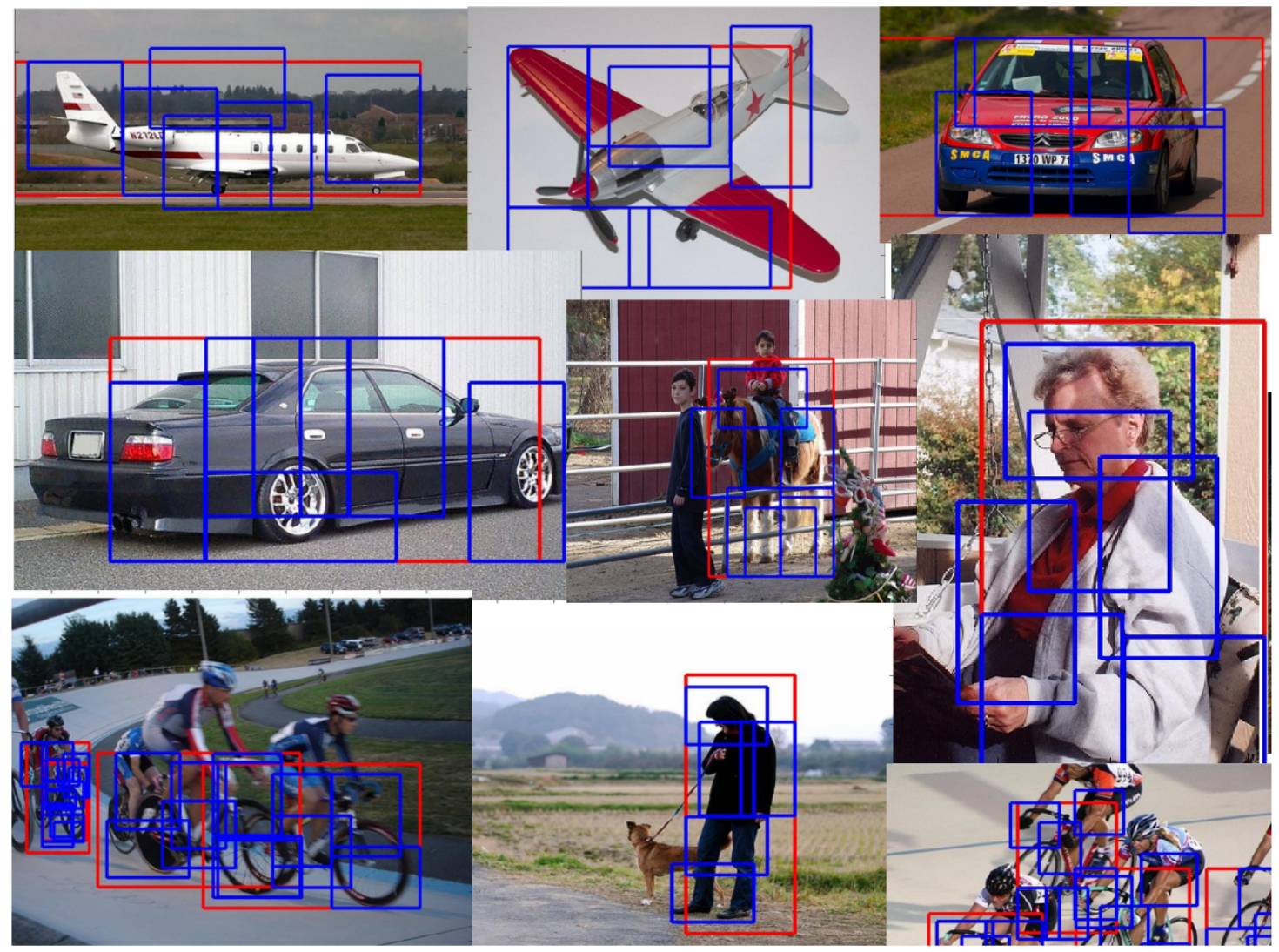

Figura 5.3: algumas detecções em PASCAL VOC 2007, usando uma MDPM de 3 componentes e 6 partes. 


\subsection{Detecção de carros em vídeos obtidos por uma câmera estaci- onária}

\subsubsection{Conjunto de dados 1 (E2)}

A descrição deste conjunto encontra-se na Subseção 4.2.1. Baseados nos resultados obtidos para a classe carro, apresentados na Tabela 5.1, foram selecionadas MDPMs de três componentes da mistura e seis, oito e dez partes do modelo para serem usadas neste experimento. Neste conjunto, foi selecionada uma região onde vai ser aplicado o detector, tal como foi apresentada na Figura 4.4b. Pelo fato de não haver informação dos bounding boxes dos carros que aparecem nos vídeos, a análise dos resultados é realizada de maneira visual.

As Figuras 5.4, 5.5, 5.6, 5.7, 5.8, 5.9, 5.10, 5.11, 5.12; apresentam resultados de alguns quadros obtidos para cada vídeo do conjunto de dados. As primeiras três colunas foram obtidas usando MDPMs simétricas e as três últimas colunas usando MDPMs assimétricas. Caso não sejam detectados bounding boxes no quadro, então não mostramos nenhum resultado e deixamos um espaço em branco.
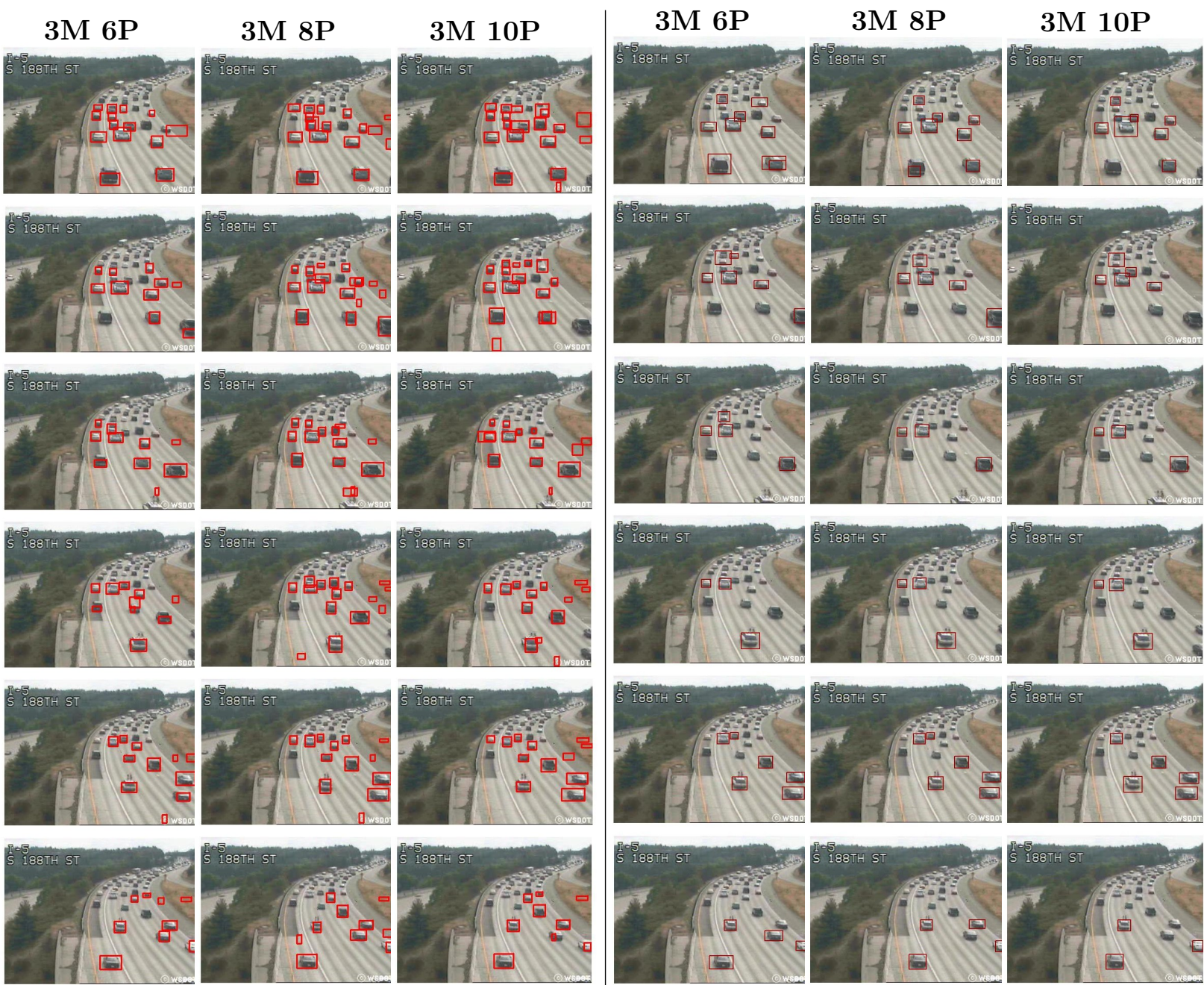

(a) MDPM simétrico

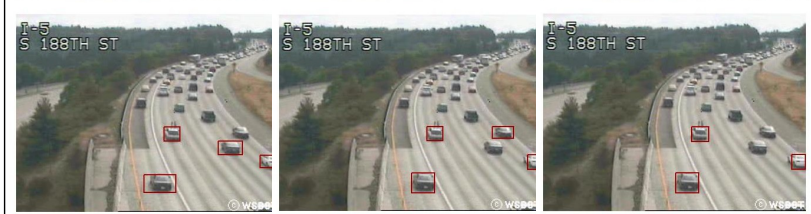

(b) MDPM assimétrico

Figura 5.4: Deteç̧ões para o vídeo V1; usando uma MDPM (a) simétrica e (b) assimétrica.

Observou-se que, usando MDPMs simétricas, em geral, resultados melhores foram obtidos. Este resultado foi definido considerando a MDPM que tem menor número de falsos positivos (FP) e também menor número de falsos negativos (FN) nos vídeos. A possível razão para isso é porque os veículos no vídeo são na sua maioria simétricos (i.e. vista da parte de trás). 


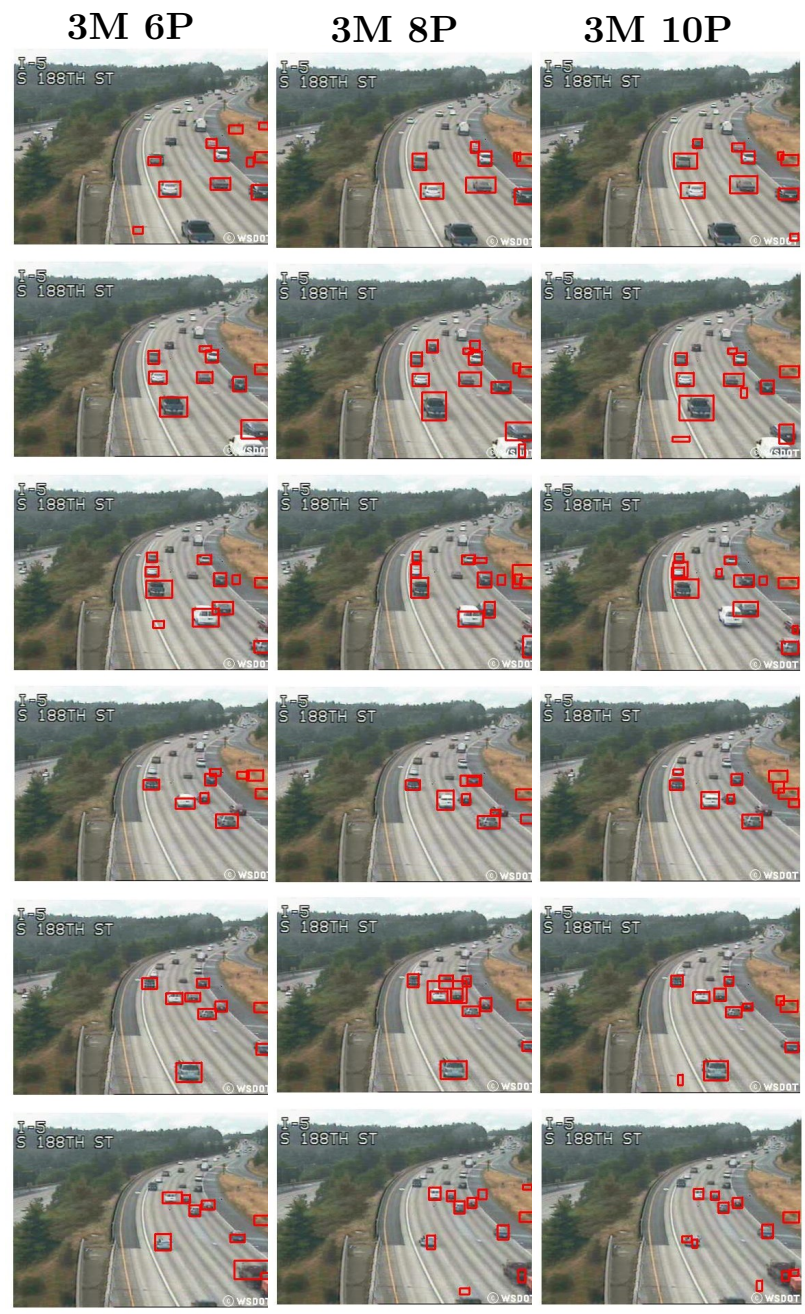

(a) MDPM simétrico
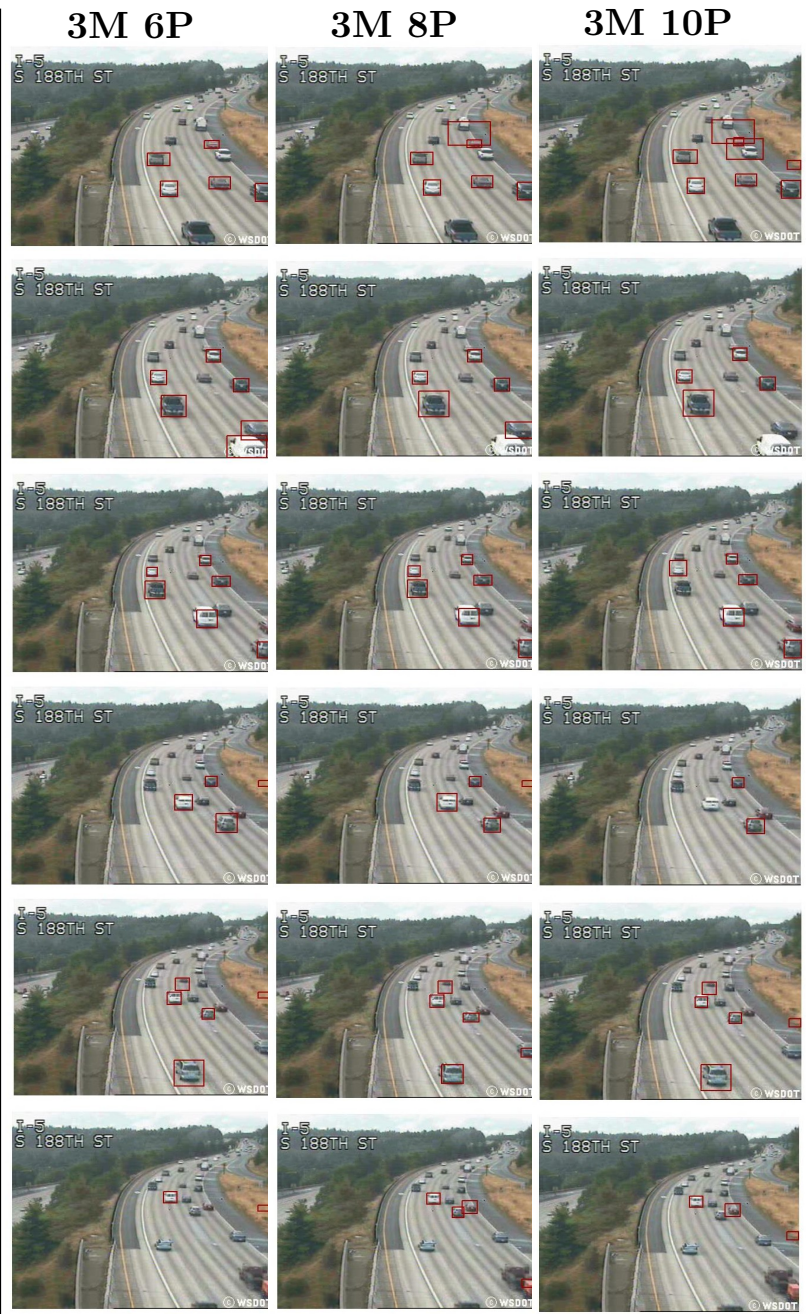

(b) MDPM assimétrico

Figura 5.5: Deteç̧̃es para o vídeo V2; usando uma MDPM (a) simétrica e (b) assimétrica.

Para os vídeos V1 e V2, eles têm menor número de FP e FN usando uma MDPM simétrica de 3 componentes e 6 partes (3M 6P). Para os vídeos V4, V8 e V9, eles têm um número menor de FP e FN usando uma MDPM simétrica de 3M 10P. Para o V3, tem-se um número menor de FP com uma MDPM assimétrica de 3M 10P e número menor de FN com uma MDPM de 3M 6P mas, em geral, ele tem melhores resultados usando uma MDPM simétrica de 3M 6P. Para o V5, um número menor de FP foi obtido com uma MDPM assimétrica de 3M6P e um número menor de FN e, em geral, melhores resultados foram obtidos com uma MDPM simétrica de 3M 6P. Para o V6, melhores resultados foram obtidos usando uma MDPM simétrica de 3M 8P e para o V7 usando uma MDPM simétrica de 3M 8P.

Com respeito aos dias mais claros, o principal motivo para ter maior numero de falsos negativos foi as sombras geradas pelos veículos (para o caso do V5 mostrados na Figura 5.8). Finalmente, observando todos os resultados, o vídeo onde foram obtidos melhores resultados foi no V7, usando uma MDPM de 3 componentes e 8 partes (3M 8P). 


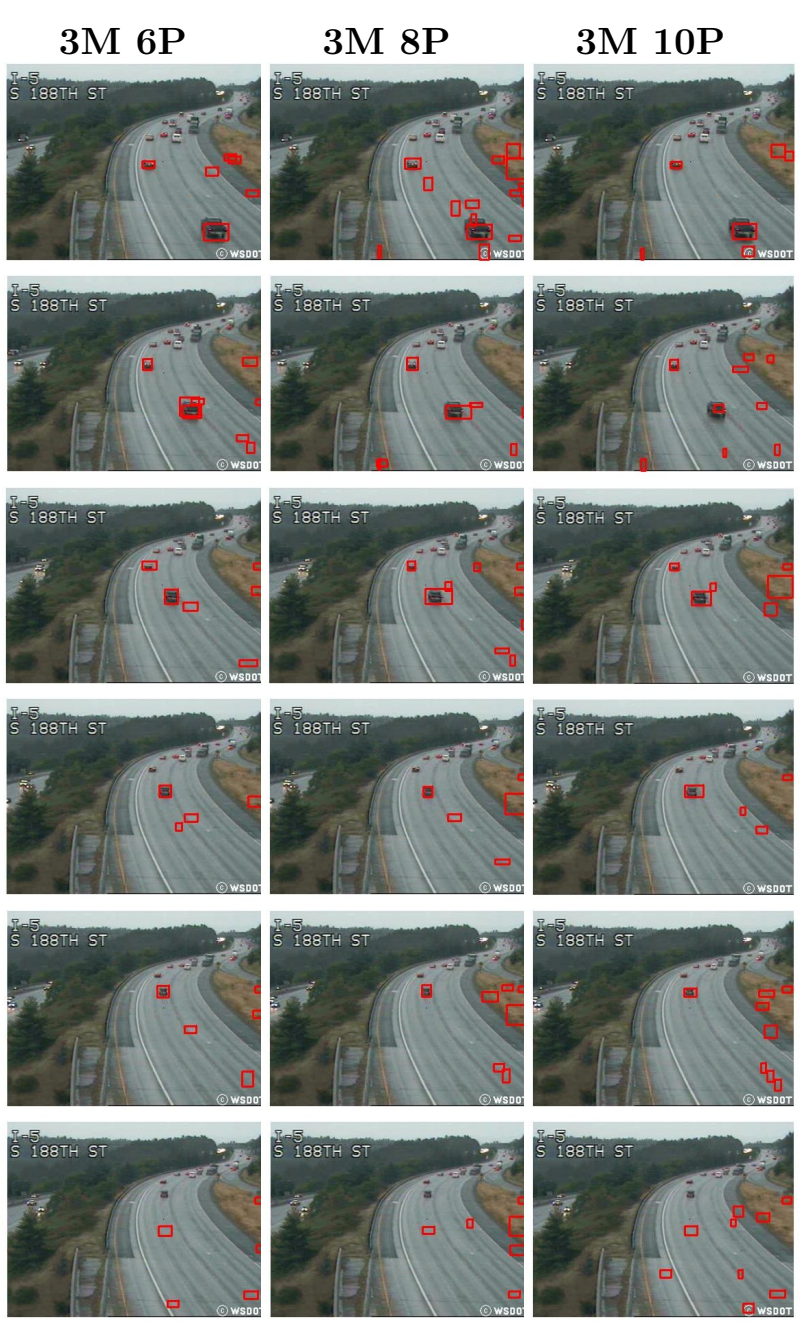

(a) MDPM simétrico
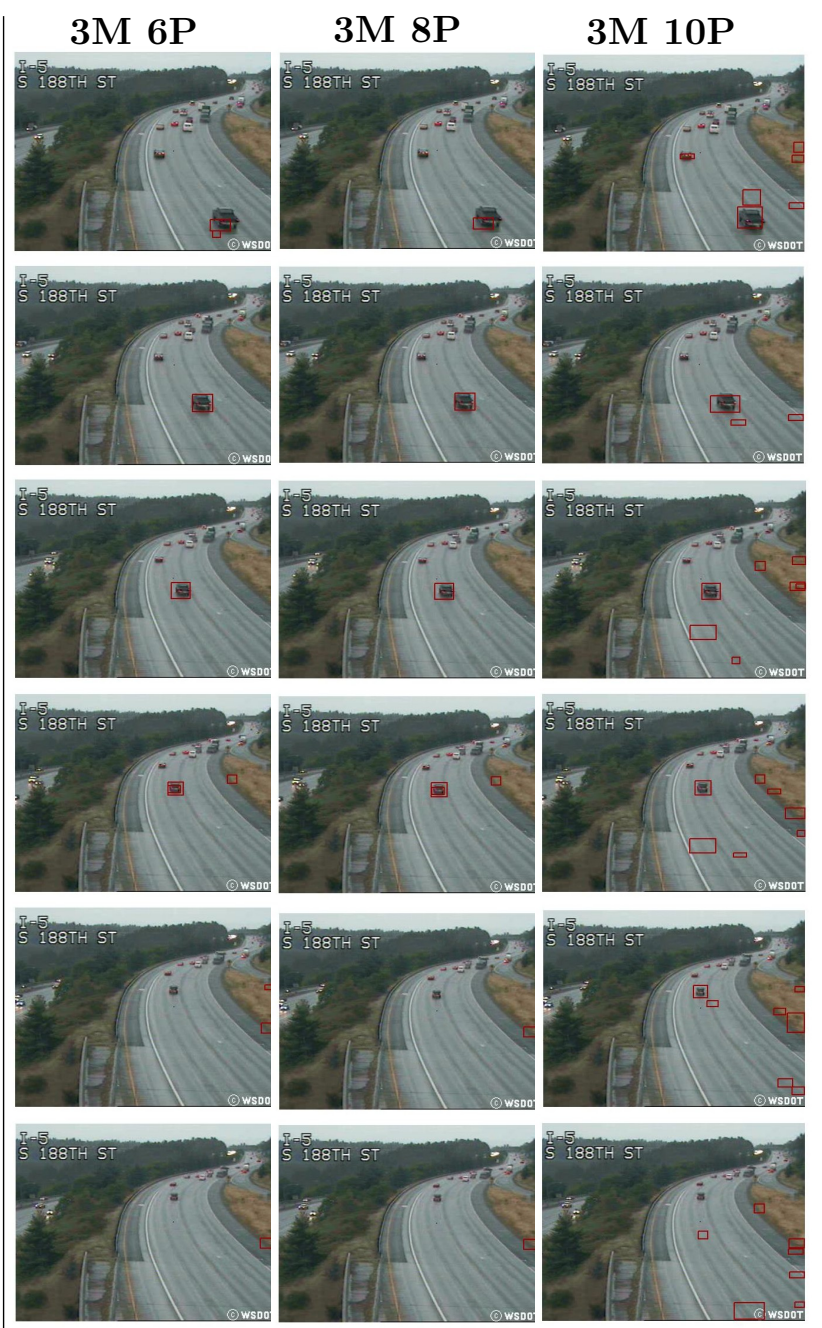

(b) MDPM assimétrico

Figura 5.6: Deteç̧ões para o vídeo V3; usando uma MDPM (a) simétrica e (b) assimétrica. 

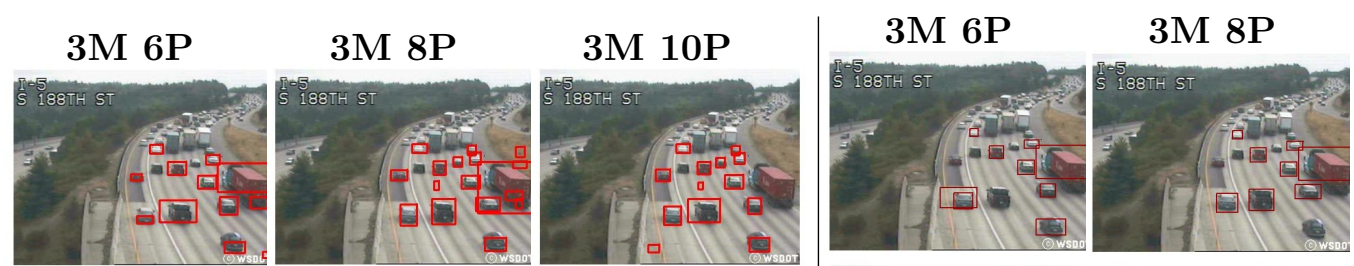

$3 \mathrm{M} 10 \mathrm{P}$
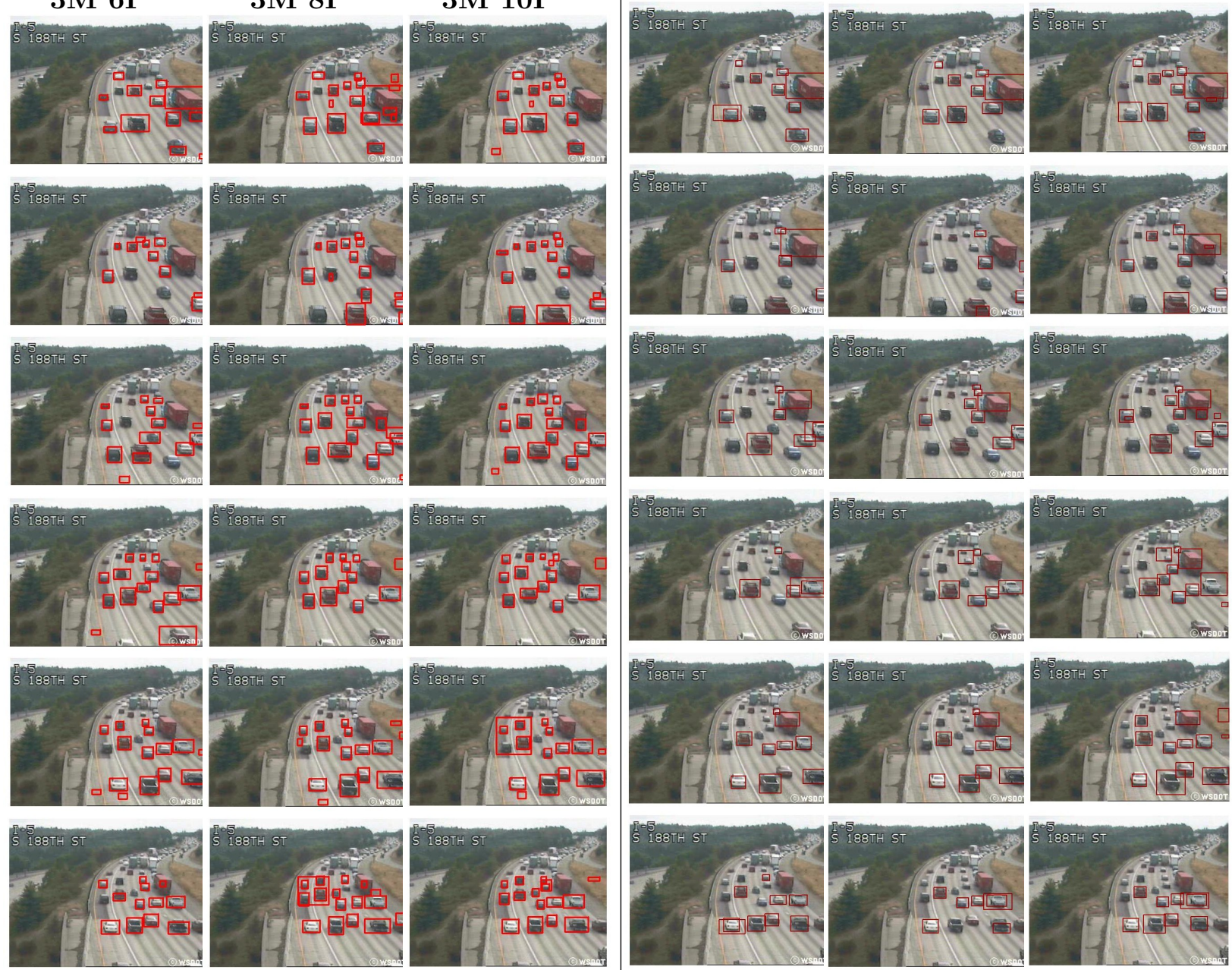

(a) MDPM simétrico

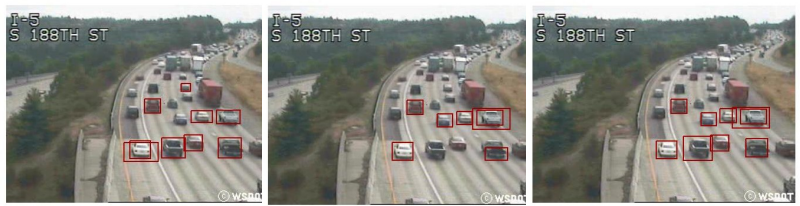

(b) MDPM assimétrico

Figura 5.7: Detecçôes para o vídeo V4; usando uma MDPM (a) simétrica e (b) assimétrica. 

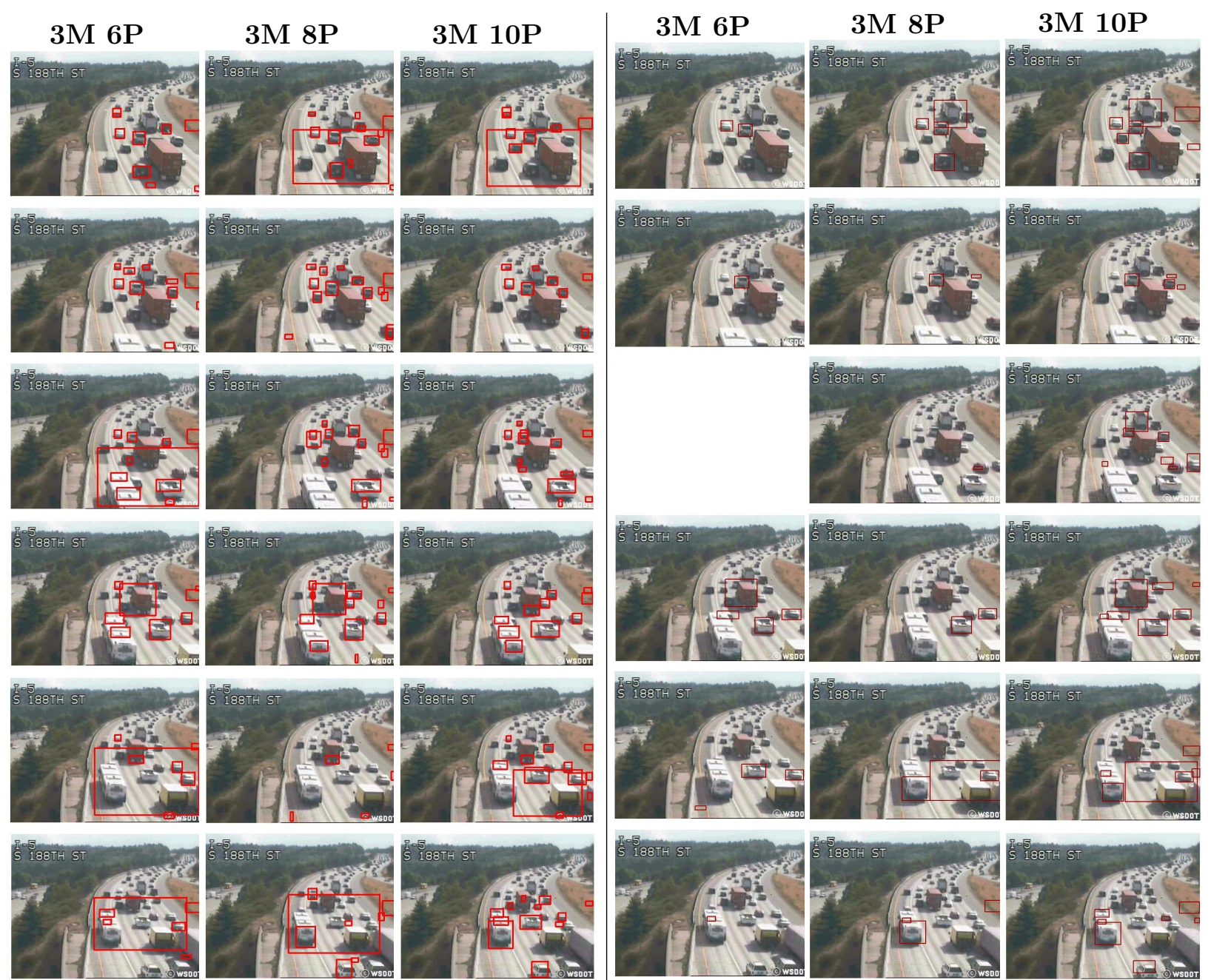

(a) MDPM simétrico
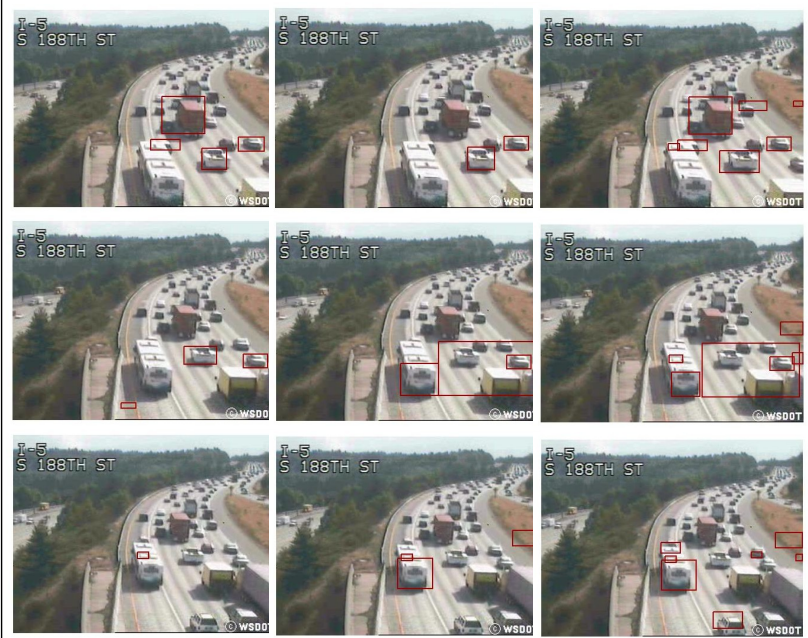

(b) MDPM assimétrico

Figura 5.8: Deteç̧ões para o vídeo V5; usando uma MDPM (a) simétrica e (b) assimétrica. 

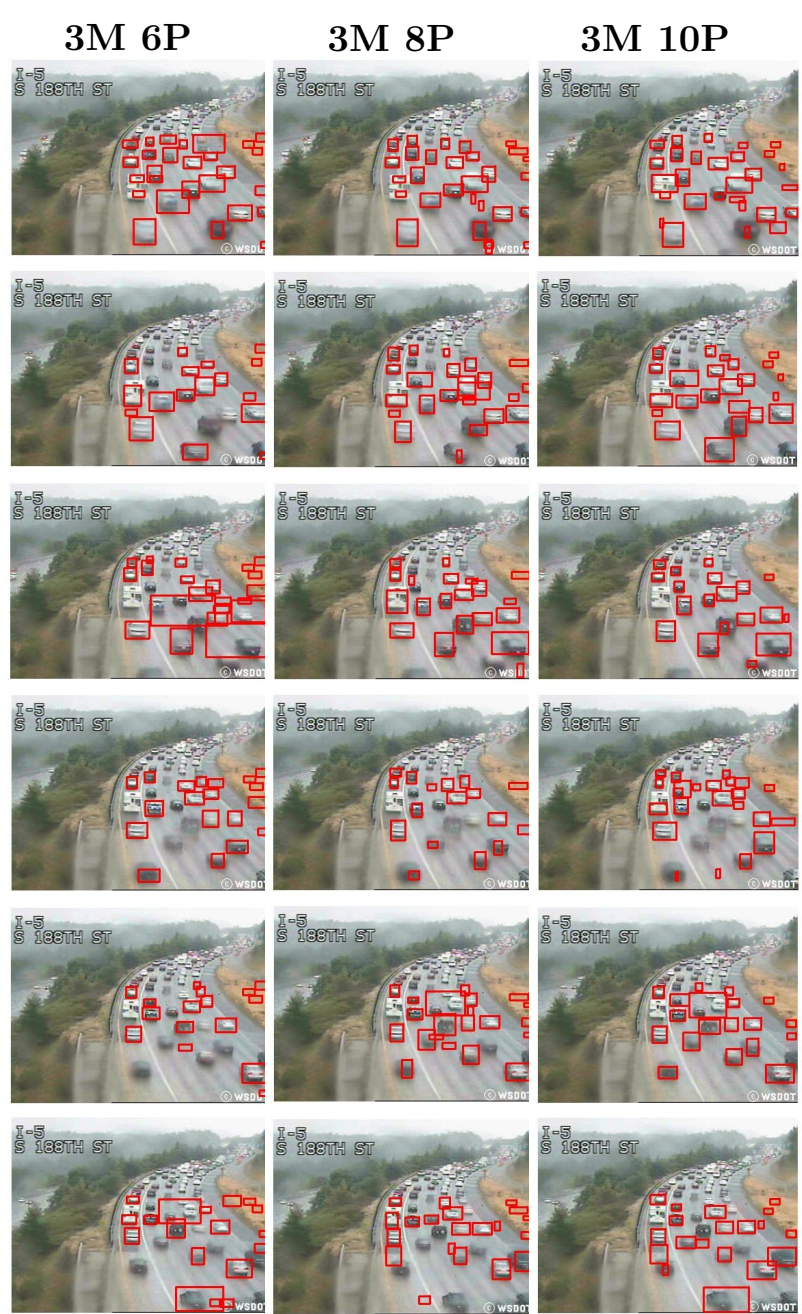

(a) MDPM simétrico

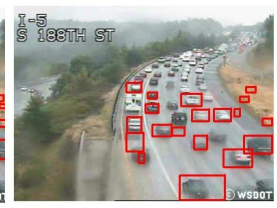

단
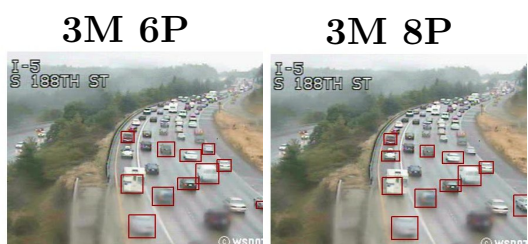

$3 \mathrm{M} 10 \mathrm{P}$
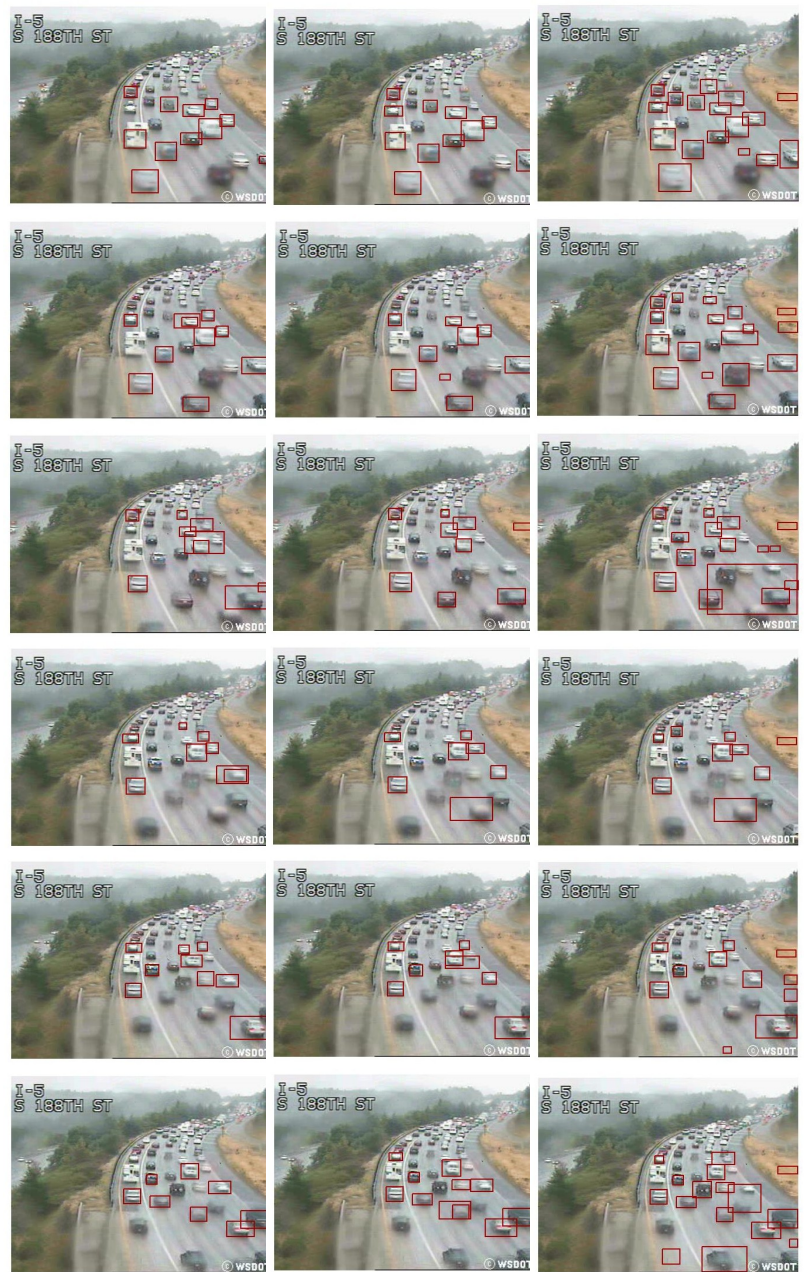

(b) MDPM assimétrico

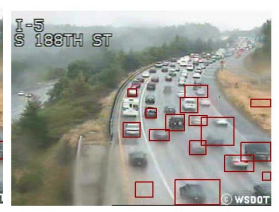

Figura 5.9: Detecções para o vídeo V6; usando uma MDPM (a) simétrica e (b) assimétrica. 

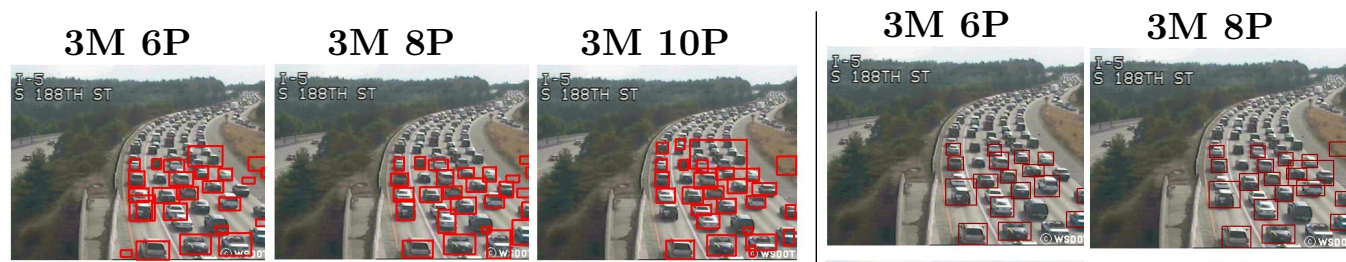

$3 \mathrm{M} 10 \mathrm{P}$
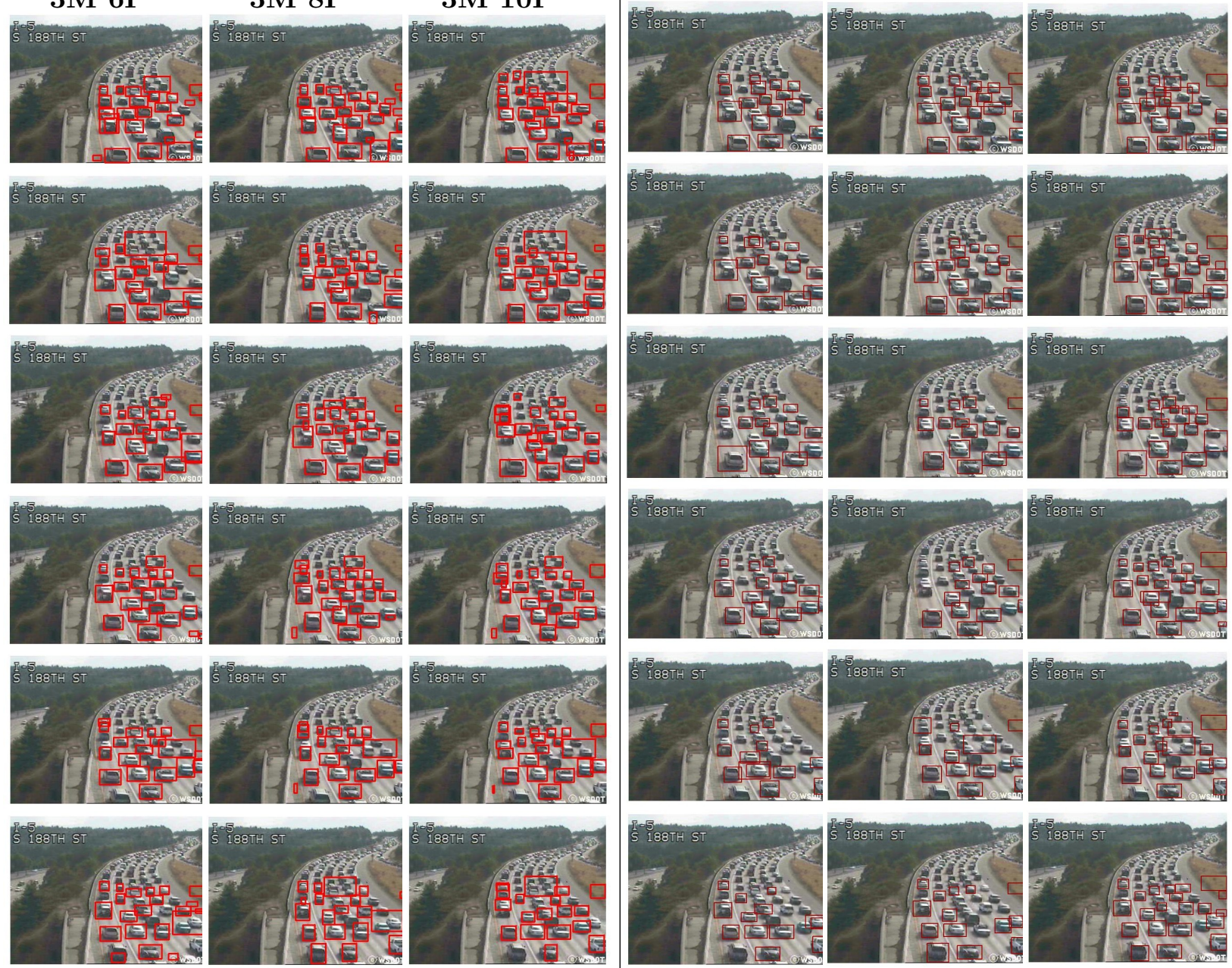

(a) MDPM simétrico

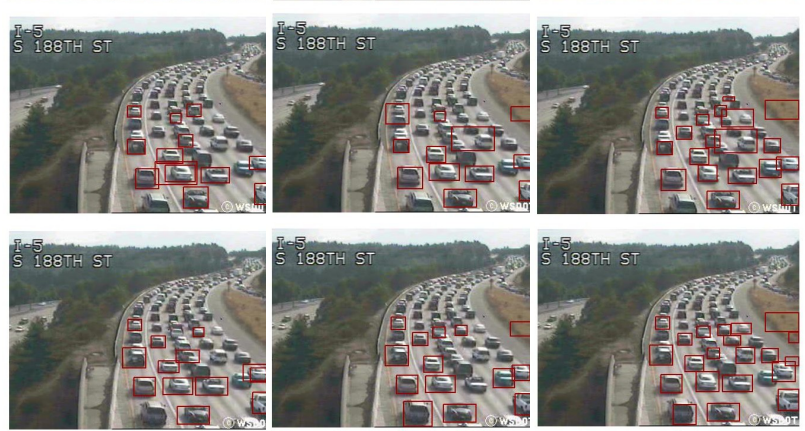

(b) MDPM assimétrico

Figura 5.10: Deteç̧ões para o vídeo V\%; usando uma MDPM (a) simétrica e (b) assimétrica. 

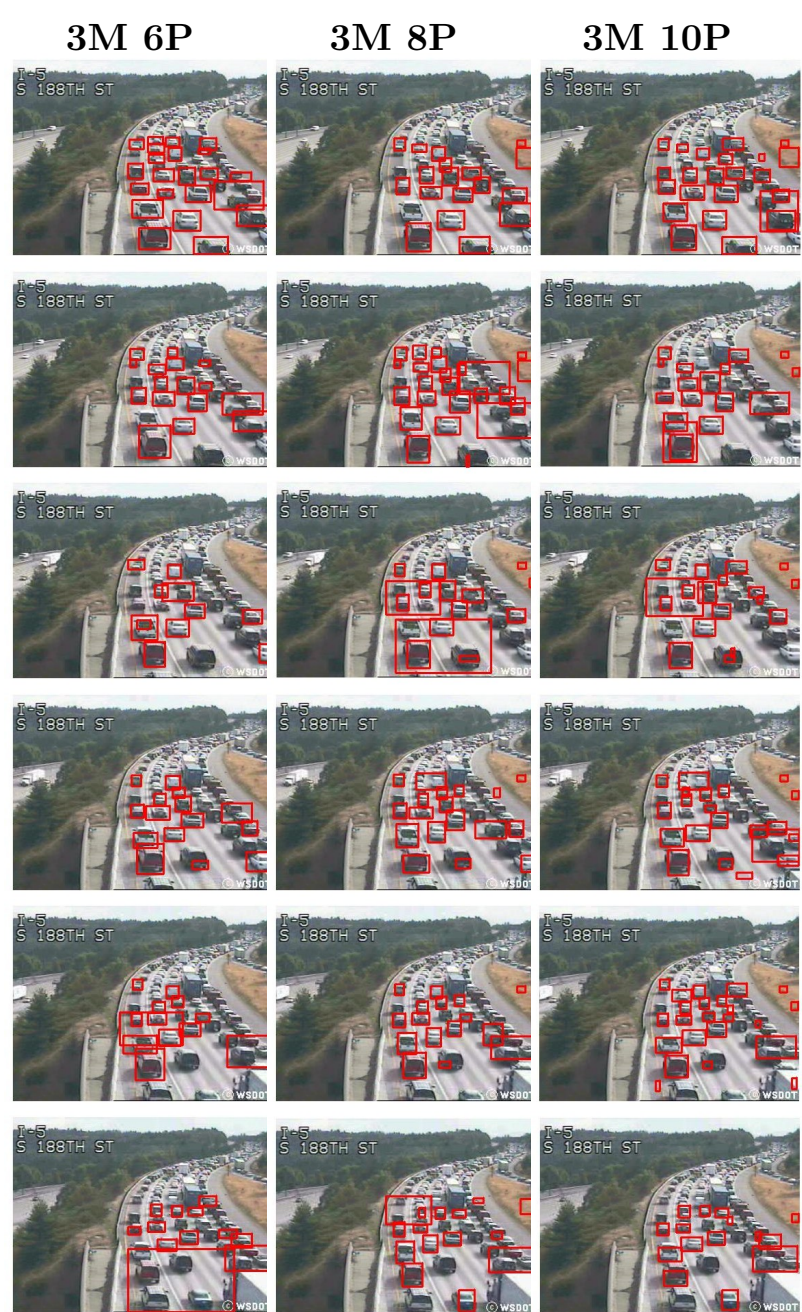

(a) MDPM simétrico
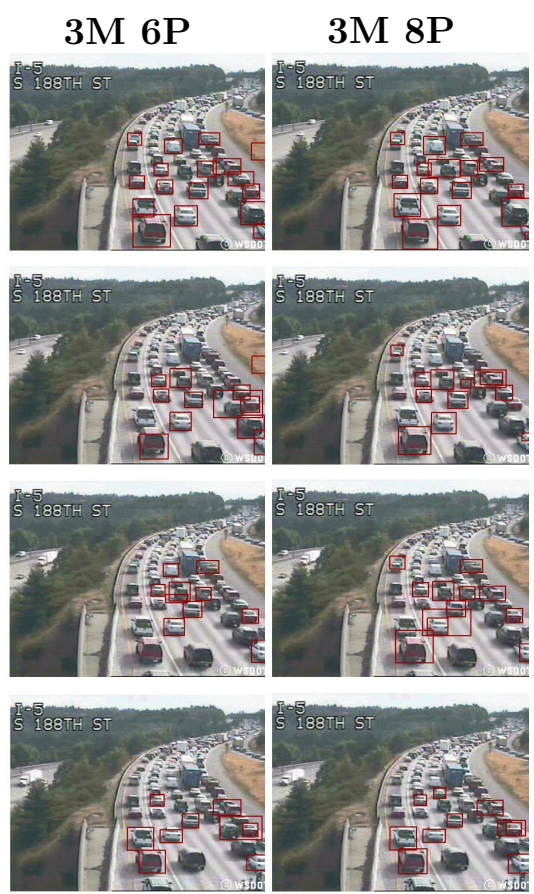

$3 \mathrm{M} 10 \mathrm{P}$
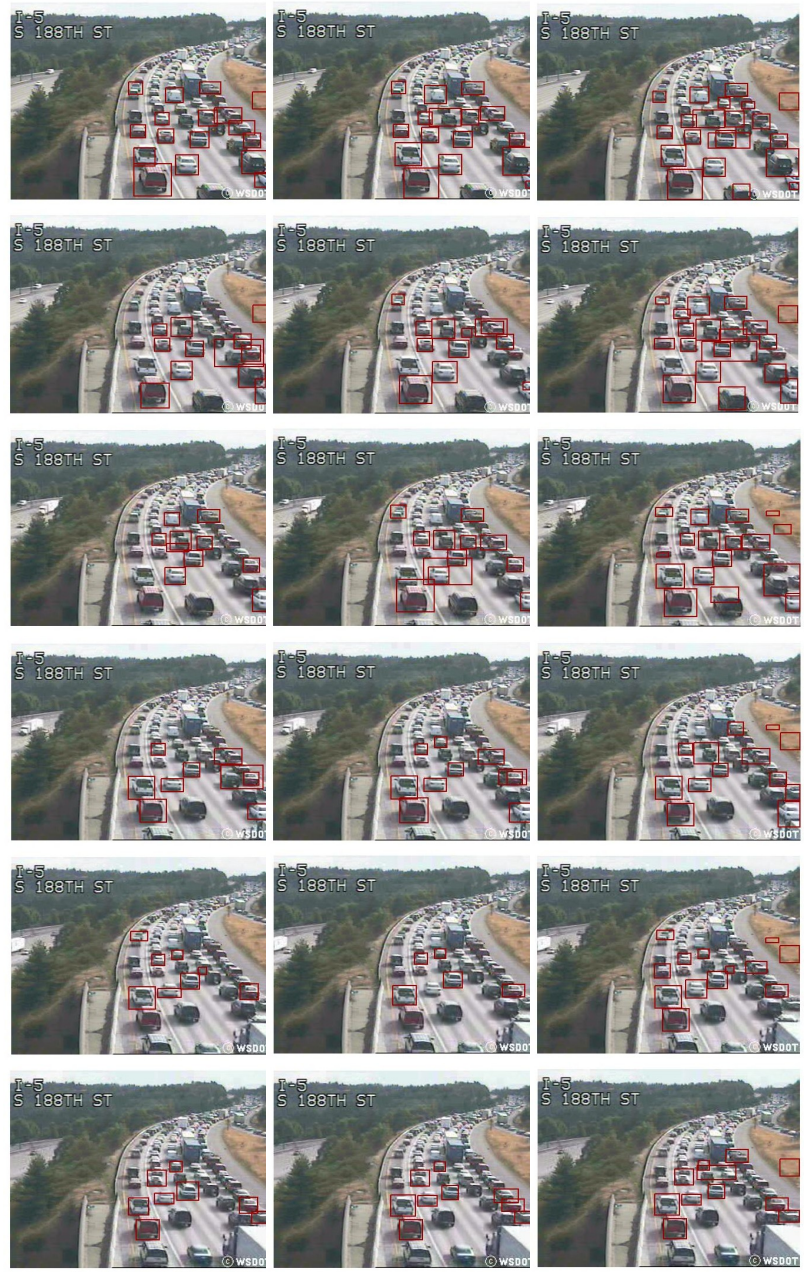

(b) MDPM assimétrico

Figura 5.11: Deteç̧ões para o vídeo V8; usando uma MDPM (a) simétrica e (b) assimétrica. 

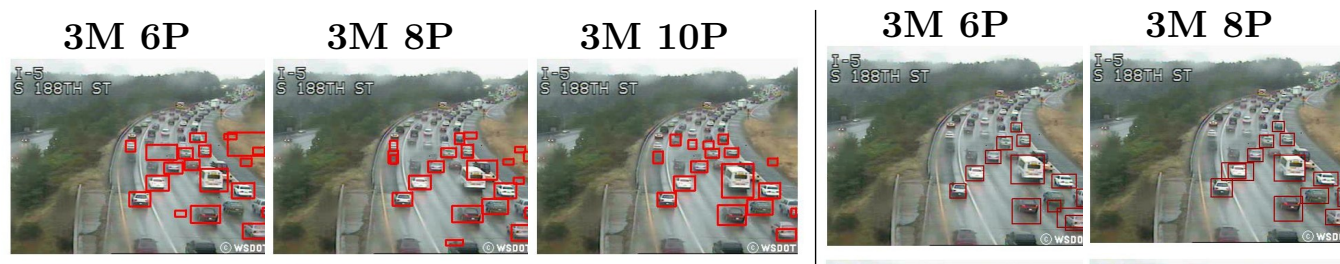

$3 \mathrm{M} 10 \mathrm{P}$
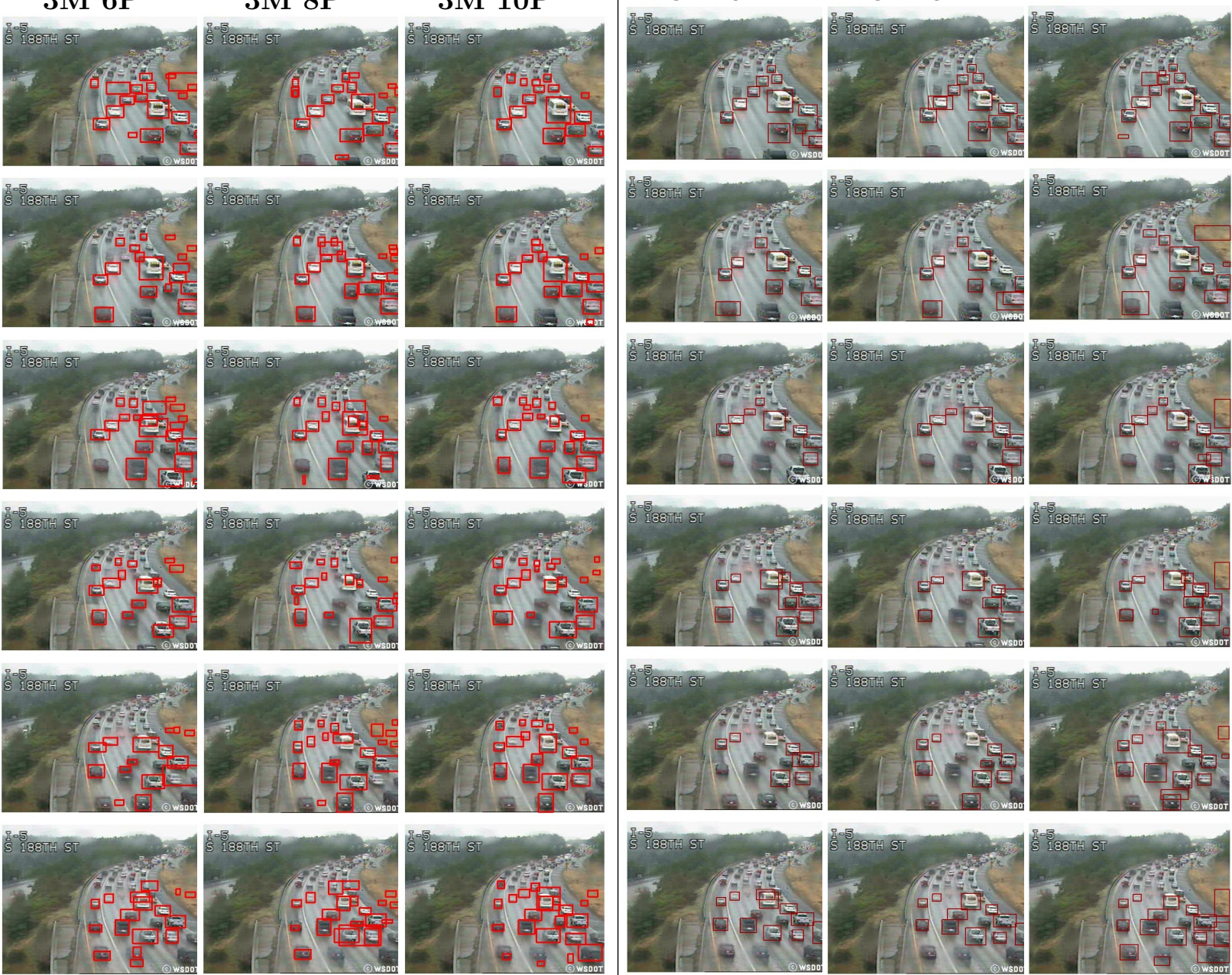

(a) MDPM simétrico

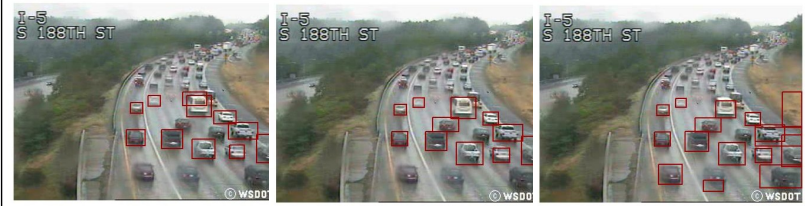

(b) MDPM assimétrico

Figura 5.12: Deteç̧ões para o vídeo V9; usando uma $M D P M$ (a) simétrica e (b) assimétrica. 


\subsubsection{Conjunto de dados 2 (E3)}

A descrição deste conjunto de dados encontra-se na Subseção 4.2.1. Não encontra-se disponível os bounding boxes do vídeo, mas, como descrito no capítulo anterior, criamos groundtruths para algumas sequências dele (sequência 001, 002, 015, 026).

Testamos usando MDPMs de dois e três componentes da mistura e seis, oito e dez partes do modelo. Adicionalmente, comparamos os nossos resultados com dois modelos de carro: model1 e model2 criados por Geiger et al. (2011). Desta forma, verificamos como que se comportam os modelos treinados com imagens específicas, neste caso de sequências de vídeo obtidos por uma câmera dinâmica. Em seguida, fizemos a análise dos resultados obtidos para cada uma das sequências. Para facilitar a comparação dos resultados, os gráficos encontram-se normalizados nos eixos.

- Para $A V S S_{-} P V_{-} E V A L-001 . a v i$ :

Analisando a Figura 5.13a, dois modelos são claramente melhores que os outros $(2 M 8 P$ e $2 M 10 P$ ) para o caso simétrico. Seus APs são também melhores que os outros APs de nossos modelos e melhores que o model1 e o model2 (Figura 5.13c). Analisando a Tabela 5.3, para o caso simétrico, podemos observar que o model 1 e o model 2 tem um alto valor de FPPI em comparação com os nossos modelos. Alguns carros não foram detectados, principalmente aqueles que são muito pequenos.

A análise para o caso assimétrico é mais difícil (Figure 5.13b) porque as curvas estão, praticamente, se superpondo no gráfico. Baseados no FPPI, podemos dizer que, com $2 M 8 P$, são obtidos os melhores resultados incluindo as MDPMs simétricas e os outros modelos. Neste sentido, a Figura 5.13d confirma esta escolha. É interessante também comparar os nossos modelos com o model1 e o model2. O número de FPPI é muito pior que os nossos.

\begin{tabular}{l|cccc|cccc}
\hline & \multicolumn{6}{|c|}{ Simétrica } & \multicolumn{4}{c}{ Assimétrica } \\
MDPM & AP & Prec. & Recall & FPPI & AP & Prec. & Recall & FPPI \\
\hline $2 M 6 P$ & 0.519 & 0.403 & 0.646 & 2.541 & 0.689 & 0.659 & 0.742 & 1.021 \\
$2 M 8 P$ & 0.636 & 0.384 & 0.724 & 3.089 & 0.688 & 0.700 & 0.725 & 0.826 \\
$2 M 10 P$ & 0.635 & 0.395 & 0.724 & 2.955 & 0.687 & 0.655 & 0.729 & 1.021 \\
$3 M 6 P$ & 0.516 & 0.273 & 0.745 & 5.282 & 0.674 & 0.549 & 0.748 & 1.632 \\
$3 M 8 P$ & 0.522 & 0.317 & 0.767 & 4.394 & 0.684 & 0.528 & 0.755 & 1.795 \\
$3 M 10 P$ & 0.515 & 0.299 & 0.753 & 4.690 & 0.671 & 0.504 & 0.758 & 1.983 \\
\hline model 1 & 0.599 & 0.135 & 0.727 & 12.349 & - & - & - & - \\
model 2 & 0.611 & 0.192 & 0.740 & 8.306 & - & - & - & - \\
\hline
\end{tabular}

Tabela 5.3: AP, recall, precision e FPPI usando MDPMs simétricas e assimétricas em $A V S S_{-} P V_{-} E V A L-001 . a v i$.

A Figura 5.14 mostra os resultados obtidos para os quadros: 1, 41, 61, 81, 121, 141, 181, 221, $261,301,341,381,421,461,501,541,561,581,601,621,641,661,681,701,721$. Os bounding boxes em vermelho foram obtidos pelo sistema com a MDPM assimétrica de $2 M 8 P$; os bounding boxes em verde foram obtidos usando o model2 (Geiger et al., 2011); e os bounding boxes em azul são os bounding boxes que anotamos para a sequencia. Observa-se que bons resultados foram obtidos para as diferentes vistas de um veículo que aparecem na cena.

- Para $A V S S_{-} P V_{-} E V A L-002 . a v i$ :

Analisando a Figura 5.15 e a Tabela 5.4, temos que com respeito à curva precision-recall e APs obtidos, $2 M 8 P$ é novamente claramente melhor que os outros modelos para o caso simétrico $(A P=0.373$ e $F P P I=1.632)$. No caso das MDPMs assimétricas, dois deles são claramente melhores: $2 M 8 P(A P=0.423$ e $F P P I=0.245)$ e $2 M 10 P(A P=0.426$ e $F P P I=0.293)$. Eles também são melhores que o model1 $(A P=0.282$ e $F P P I=10.059)$, 


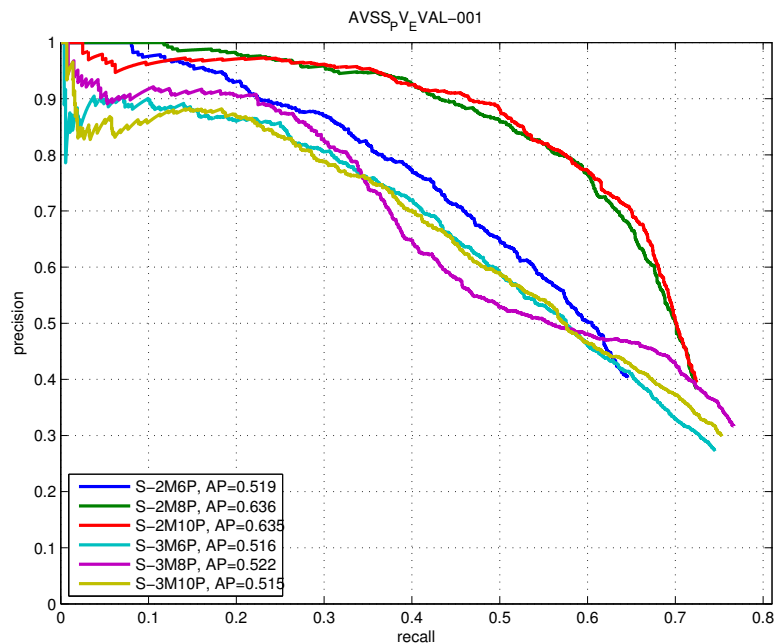

(a) Curva PR. MDPM Simétrica

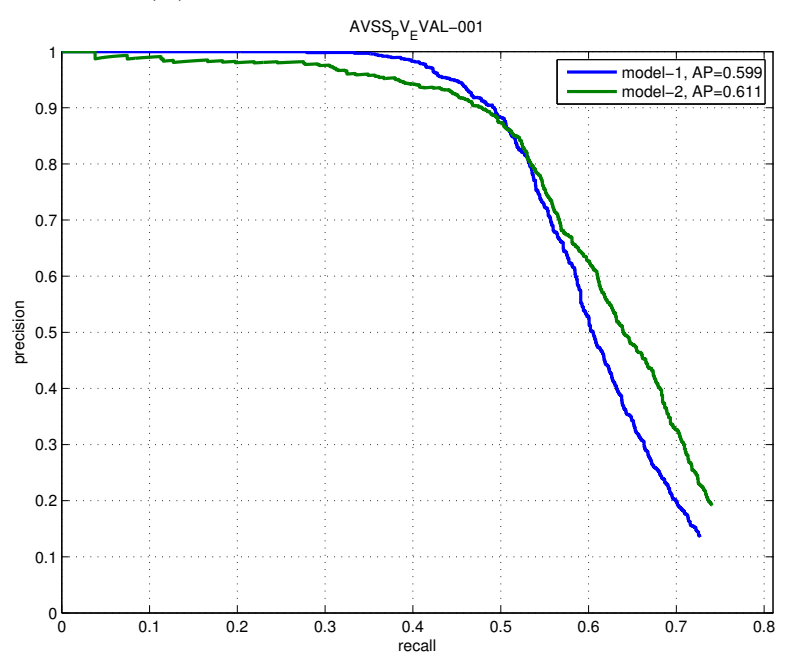

(c) Curva PR. Outros dois modelos

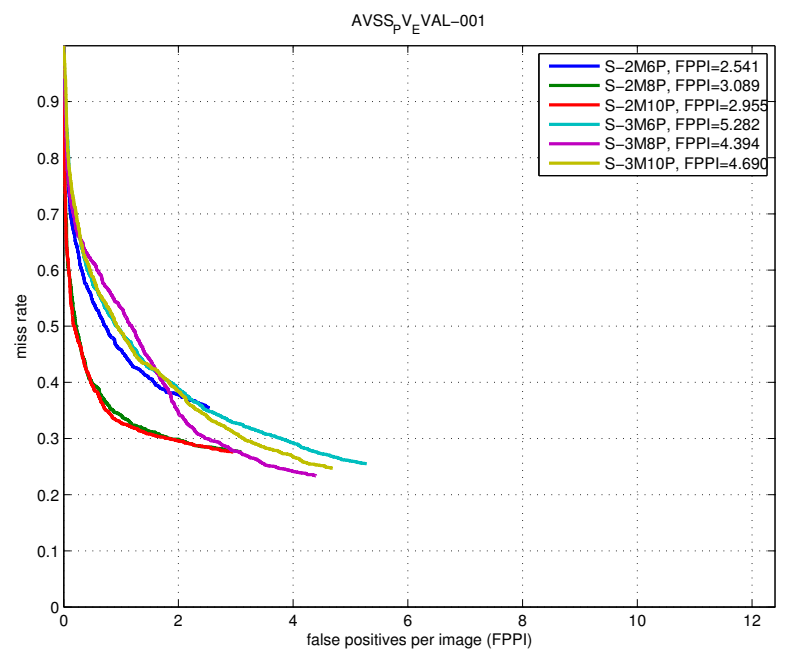

(e) Curva FPPI. MDPM Simétrica

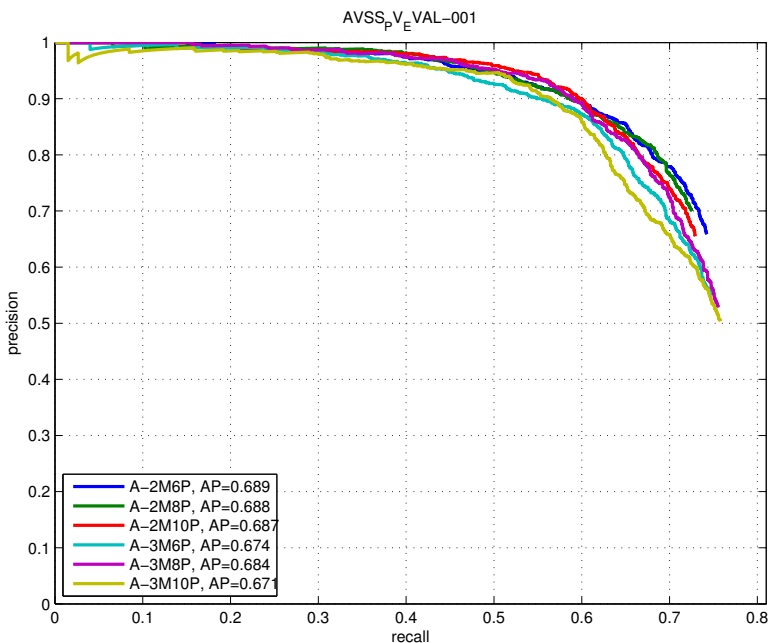

(b) Curva PR. MDPM Assimétrica

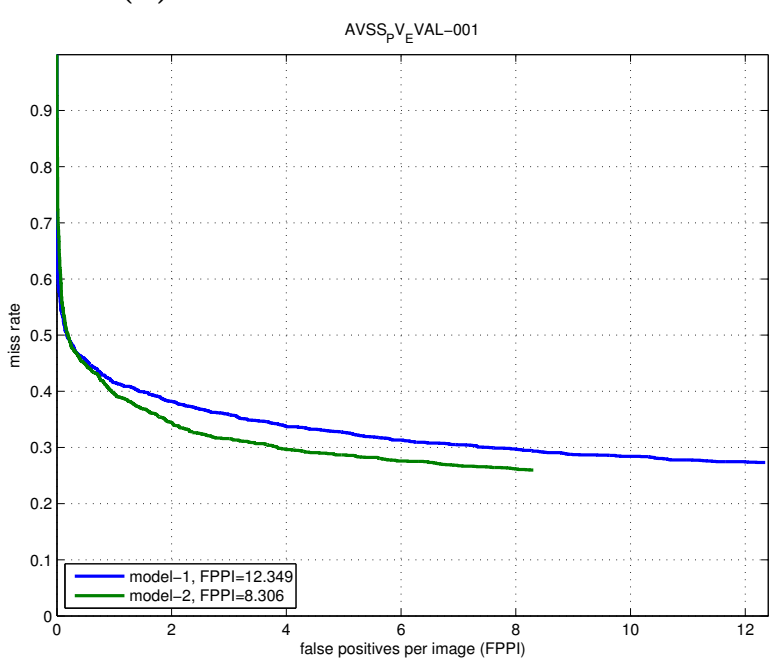

(d) Curva FPPI. Outros dois modelos

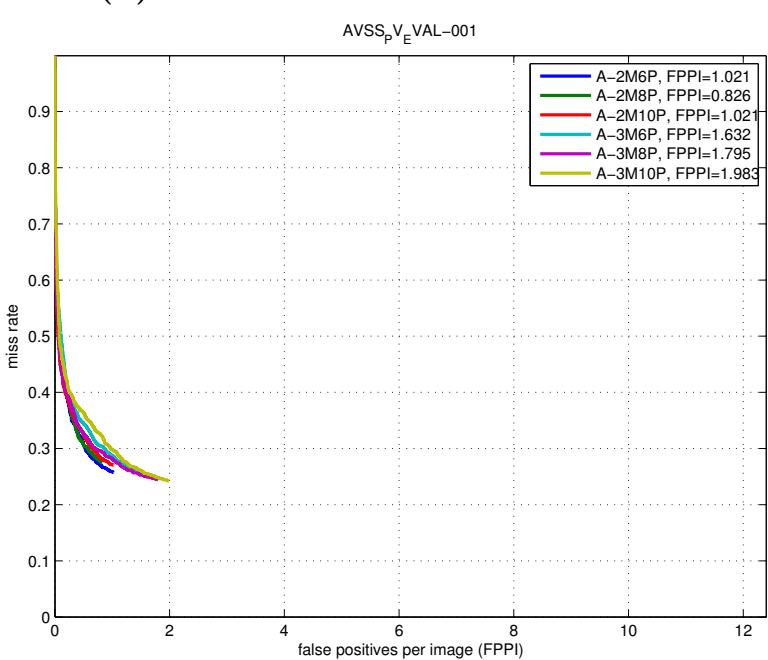

(f) Curva FPPI. MDPM Assimétrica

Figura 5.13: Curva precision vs. recall (PR) curva e curva miss rate vs. FPPI em AVSS_PV_EVAL001.avi. 


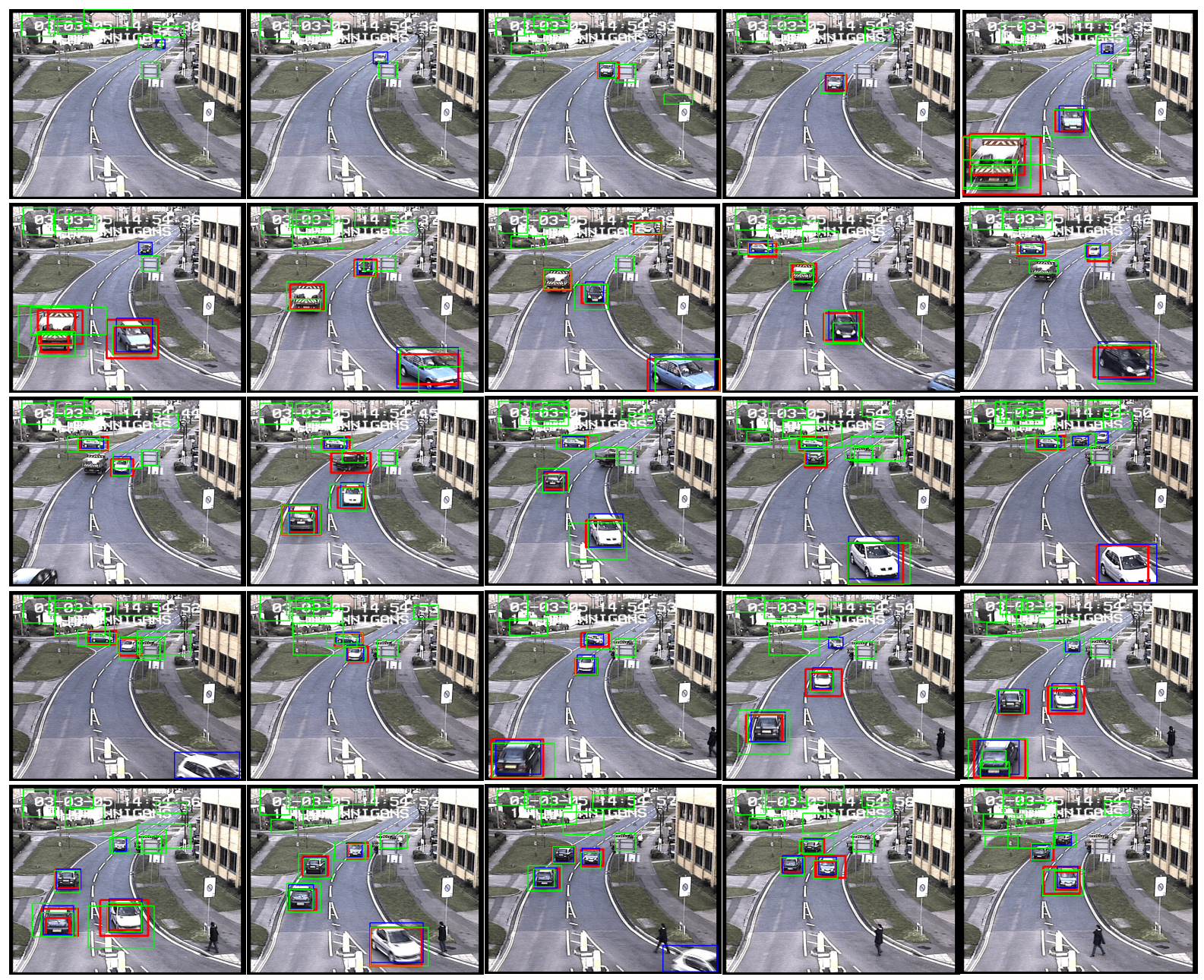

Figura 5.14: Resultado usando uma MDPM assimétrica de dois componentes e oito partes e o model2, em $A V S S_{-} P V_{-} E V A L-001 . a v i$.

o model2 $(A P=0.273$ e $F P P I=6.193)$ e todas as outras MDPMs simétricas testadas. Em relação aos APs, o melhor resultado foi obtido por $2 M 10 P$ para o caso assimétrico. Contudo, $2 M 8 P$ obtive o menor número de FPPI. É interessante comparar que o model1 e o model 2 tem piores FPPI que as nossas MDPMs.

\begin{tabular}{l|cccc|cccc}
\hline & \multicolumn{5}{|c|}{ Symmetric } & \multicolumn{4}{c}{ Asymmetric } \\
MDPM & AP & Prec. & Recall & FPPI & AP & Prec. & Recall & FPPI \\
\hline $2 M 6 P$ & 0.268 & 0.340 & 0.319 & 1.208 & 0.423 & 0.679 & 0.408 & 0.375 \\
$2 M 8 P$ & 0.373 & 0.326 & 0.405 & 1.632 & 0.423 & 0.762 & 0.403 & 0.245 \\
$2 M 10 P$ & 0.333 & 0.299 & 0.392 & 1.790 & 0.426 & 0.731 & 0.408 & 0.293 \\
$3 M 6 P$ & 0.235 & 0.190 & 0.427 & 3.553 & 0.420 & 0.593 & 0.419 & 0.559 \\
$3 M 8 P$ & 0.234 & 0.238 & 0.427 & 2.656 & 0.408 & 0.551 & 0.413 & 0.655 \\
$3 M 10 P$ & 0.219 & 0.208 & 0.423 & 3.138 & 0.407 & 0.565 & 0.416 & 0.625 \\
\hline model 1 & 0.282 & 0.071 & 0.392 & 10.059 & - & - & - & - \\
model 2 & 0.273 & 0.112 & 0.400 & 6.193 & - & - & - & - \\
\hline
\end{tabular}

Tabela 5.4: AP, recall, precision e FPPI usando MDPMs simétricas e assimétricas em $A V S S_{-} P V_{-} E V A L-002 . a v i$.

- For $A V S S_{-} P V_{-} E V A L-015 . a v i$ : 


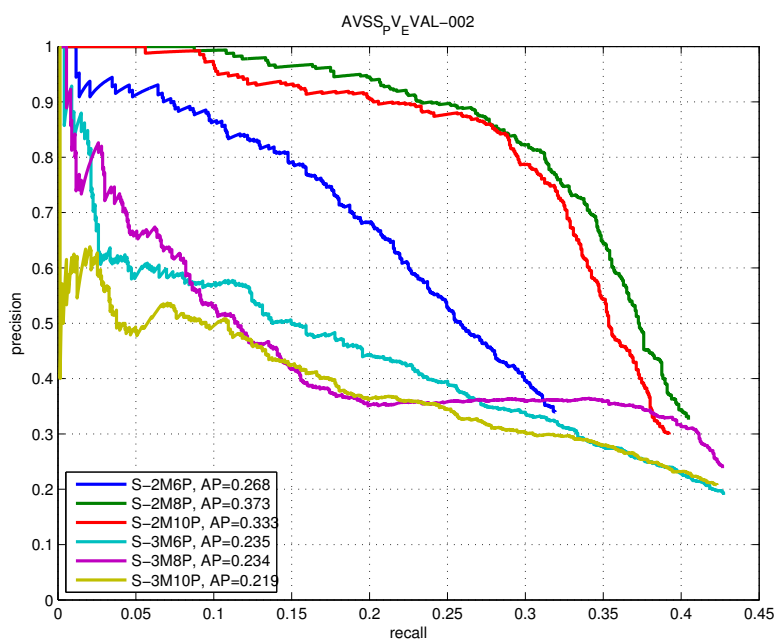

(a) Curva PR. MDPM Simétrica

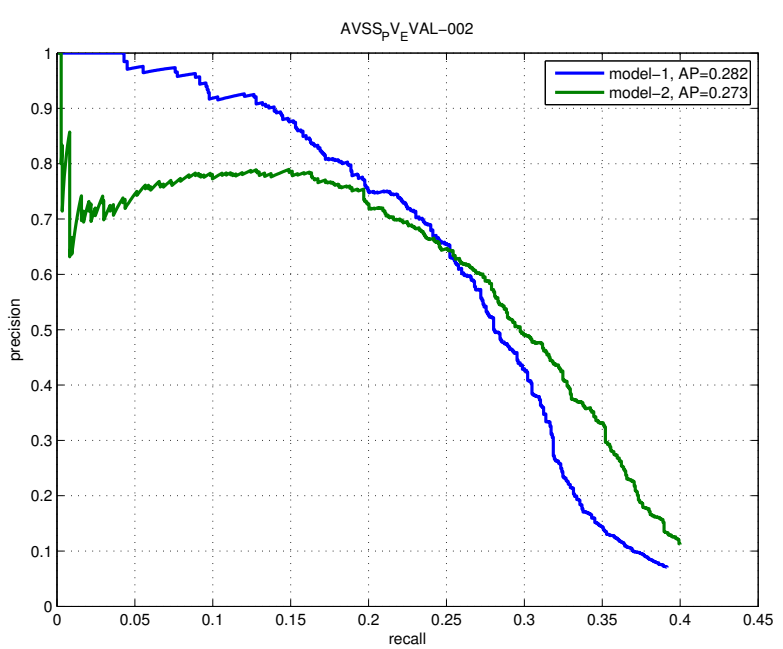

(c) Curva PR. Outros dois modelos

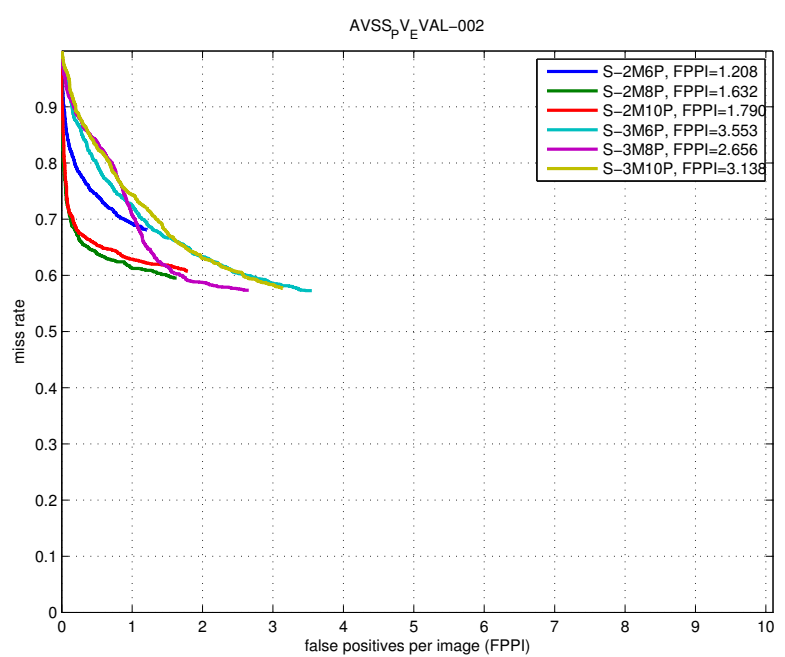

(e) Curva FPPI. MDPM Simétrica

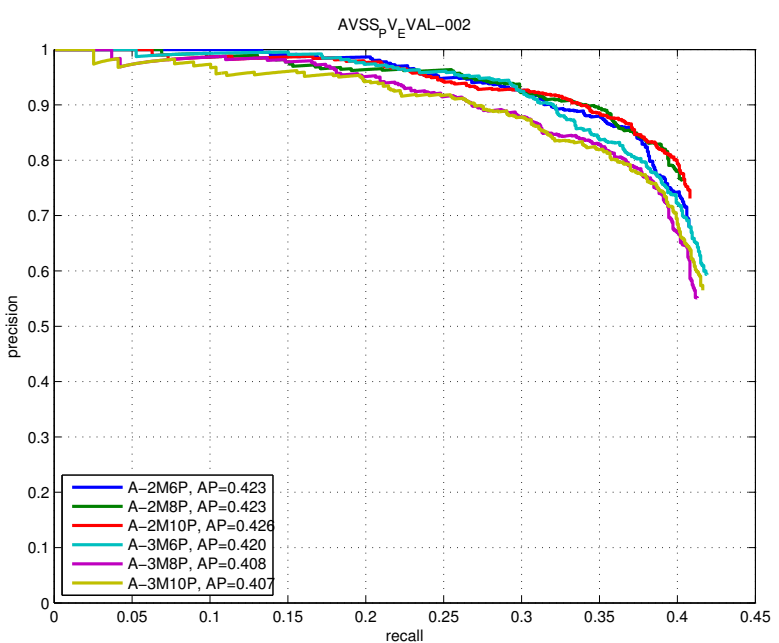

(b) Curva PR. MDPM Assimétrica

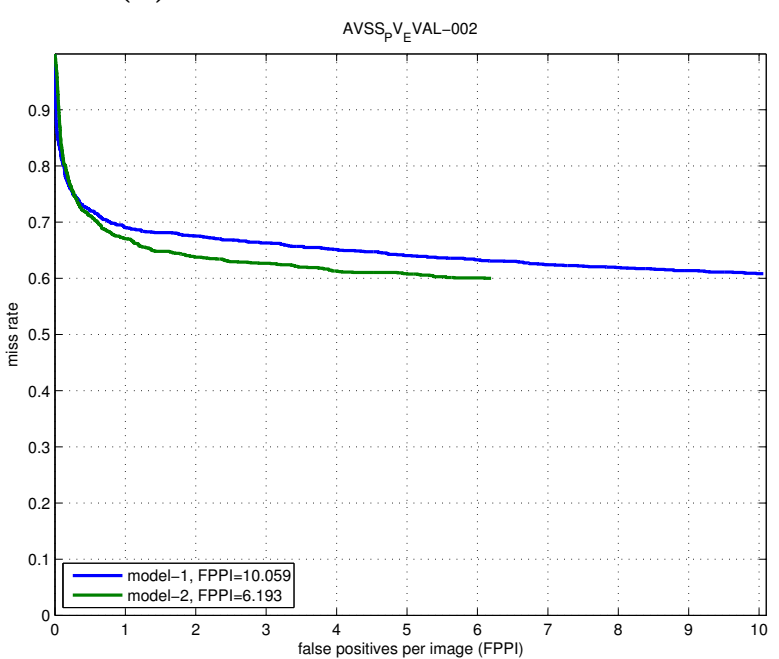

(d) Curva FPPI. Outros dois modelos

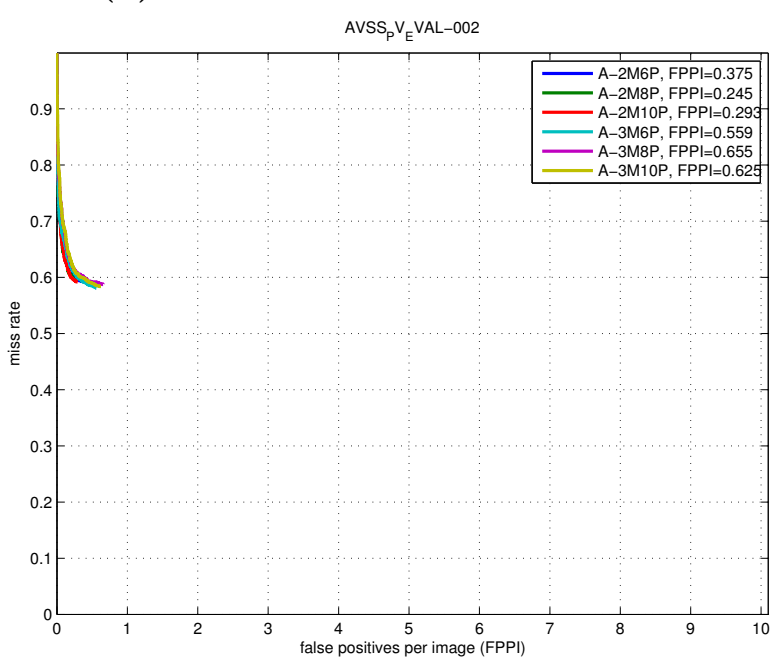

(f) Curva FPPI. MDPM Assimétrica

Figura 5.15: Curva precision vs. recall (PR) curva e curva miss rate vs. FPPI em AVSS_PV_EVAL002.avi. 
Analisando a Figura 5.16 e a Tabela 5.5, temos que, com respeito à curva precision-recall e APs, a MDPM de $2 M 8 P(A P=0.472$ e $F P P I=3.77)$ é melhor no caso simétrico. Analisando as MDPMs assimétricas, $3 M 6 P(A P=0.597$ e $F P P I=1.475)$ é melhor que o model 1 $(A P=0.404$ e $F P P I=11.919)$, o model $(A P=0.443$ e $F P P I=9.162)$ e todas as MDPMs simétricas. Isto pode ser confirmado pelo seu AP. Com respeito ao número de FPPI, dois modelos assimétricos, $2 M 8 P(A P=0.549$ e $F P P I=1.189)$ e $3 M 6 P$, obtiveram os menores FPPIs. Comparando eles com o model1 e model2, os FPPI são diferentes novamente.

\begin{tabular}{l|cccc|cccc}
\hline & \multicolumn{5}{|c|}{ Symmetric } & \multicolumn{4}{c}{ Asymmetric } \\
MDPM & AP & Prec. & Recall & FPPI & AP & Prec. & Recall & FPPI \\
\hline $2 M 6 P$ & 0.406 & 0.382 & 0.529 & 1.949 & 0.464 & 0.468 & 0.699 & 1.811 \\
$2 M 8 P$ & 0.472 & 0.295 & 0.693 & 3.770 & 0.549 & 0.575 & 0.704 & 1.189 \\
$2 M 10 P$ & 0.465 & 0.328 & 0.695 & 3.249 & 0.521 & 0.471 & 0.706 & 1.806 \\
$3 M 6 P$ & 0.285 & 0.213 & 0.672 & 5.651 & 0.597 & 0.521 & 0.703 & 1.475 \\
$3 M 8 P$ & 0.382 & 0.268 & 0.733 & 4.555 & 0.573 & 0.462 & 0.714 & 1.900 \\
$3 M 10 P$ & 0.360 & 0.269 & 0.724 & 4.477 & 0.555 & 0.459 & 0.711 & 1.908 \\
\hline model 1 & 0.404 & 0.100 & 0.583 & 11.919 & - & - & - & - \\
model 2 & 0.443 & 0.141 & 0.661 & 9.162 & - & - & - & - \\
\hline
\end{tabular}

Tabela 5.5: AP, recall, precision e FPPI usando MDPMs simétricas e assimétricas em $A V S S_{-} P V_{-} E V A L-015$.avi.

- For $A V S S_{-} P V_{-} E V A L-026 . a v i$ :

Analisando a Figura 5.17 e a Tabela 5.6, temos, mais uma vez, que com $2 M 8 P(A P=0.595$ e $F P P I=3.909)$ foi obtido o melhor AP para o caso assimétrico e com $3 M 8 P(A P=0.0 .648$ e $F P P I=2.718)$ para o caso assimétrico.

\begin{tabular}{l|cccc|cccc}
\hline & \multicolumn{5}{|c|}{ Symmetric } & \multicolumn{4}{c}{ Asymmetric } \\
MDPM & AP & Prec. & Recall & FPPI & AP & Prec. & Recall & FPPI \\
\hline $2 M 6 P$ & 0.459 & 0.479 & 0.547 & 2.578 & 0.550 & 0.531 & 0.725 & 2.778 \\
$2 M 8 P$ & 0.595 & 0.446 & 0.725 & 3.909 & 0.584 & 0.630 & 0.712 & 1.818 \\
$2 M 10 P$ & 0.612 & 0.497 & 0.728 & 3.202 & 0.562 & 0.587 & 0.706 & 2.156 \\
$3 M 6 P$ & 0.404 & 0.317 & 0.663 & 6.212 & 0.636 & 0.527 & 0.714 & 2.779 \\
$3 M 8 P$ & 0.537 & 0.390 & 0.738 & 4.999 & 0.648 & 0.538 & 0.728 & 2.718 \\
$3 M 10 P$ & 0.532 & 0.391 & 0.743 & 5.016 & 0.608 & 0.493 & 0.722 & 3.220 \\
\hline model 1 & 0.524 & 0.207 & 0.664 & 11.025 & - & - & - & - \\
model 2 & 0.515 & 0.232 & 0.673 & 9.675 & - & - & - & - \\
\hline
\end{tabular}

Tabela 5.6: AP, recall, precision e FPPI usando MDPMs simétricas e assimétricas em $A V S S_{-} P V_{-} E V A L-0026 . a v i$.

Na maioria dos casos, uma MDPM assimétrica tem melhores resultados que uma simétrica. Adicionalmente, usando uma MDPM de 8 partes é possível obter melhores resultados que com outros. Por outro lado, as MDPMs simétricas mostraram melhor desempenho no caso de pontos de vista semi-laterais. A causa principal para os falsos positivos é que eles foram detectados perto da anotação de cor branca (data e tempo da captura) presente em todos os quadros do vídeo.

Para melhorar os resultados, uma consistência entre quadros pode ser feita porque, quando o carro esta estacionado, como na sequência $A V S S 15$, o mesmo carro é detectado algumas vezes e outras não. 


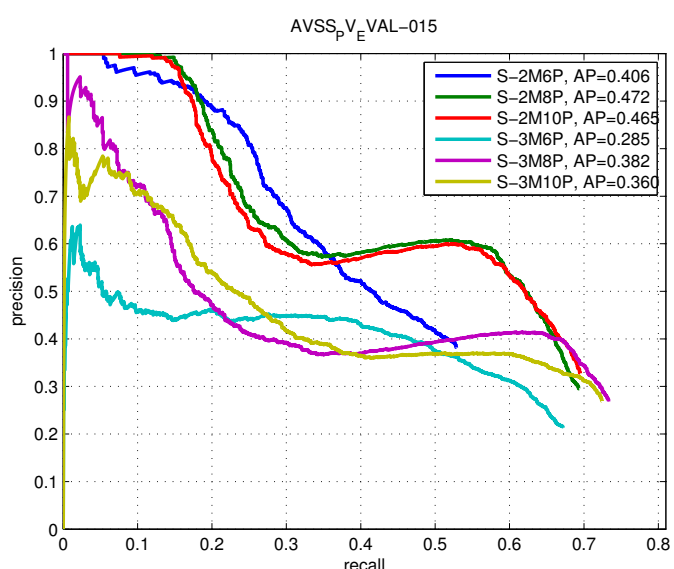

(a) Curva PR. MDPM Simétrica

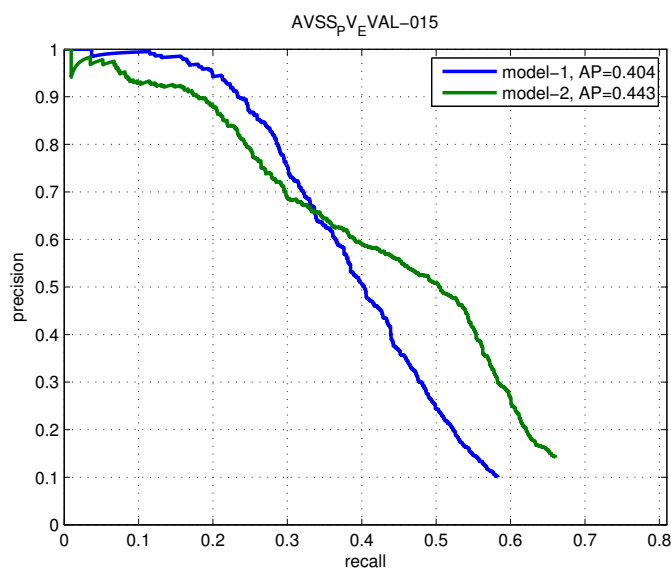

(c) Curva PR. Outros dois modelos

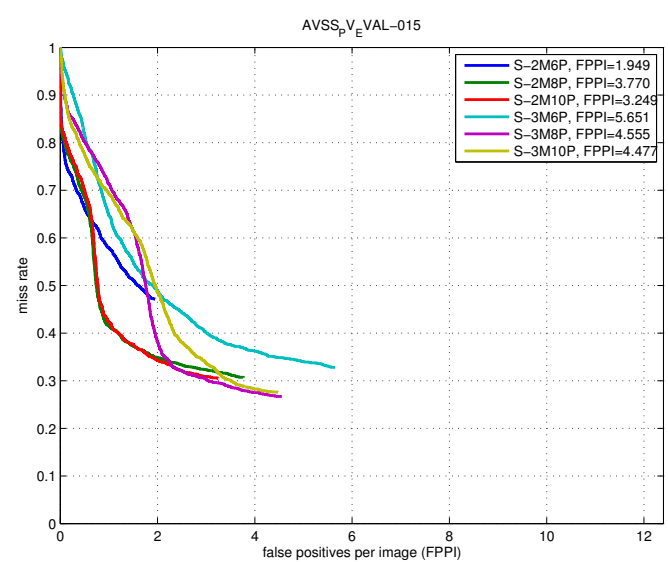

(e) Curva FPPI. MDPM Simétrica

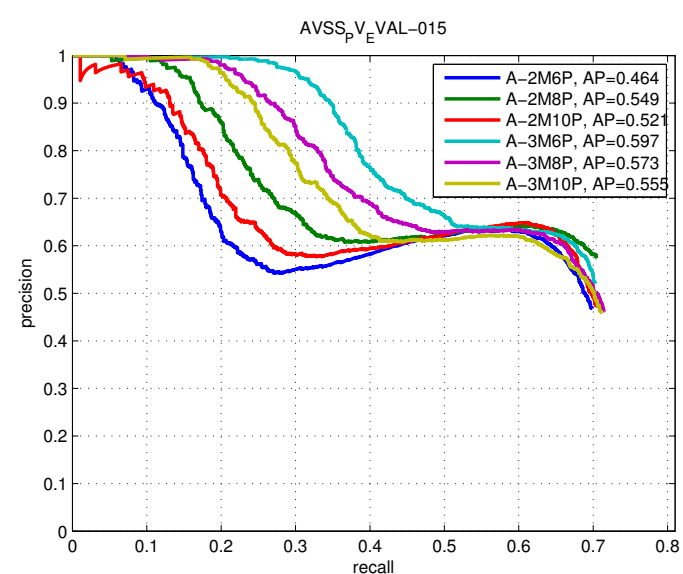

(b) Curva PR. MDPM Assimétrica

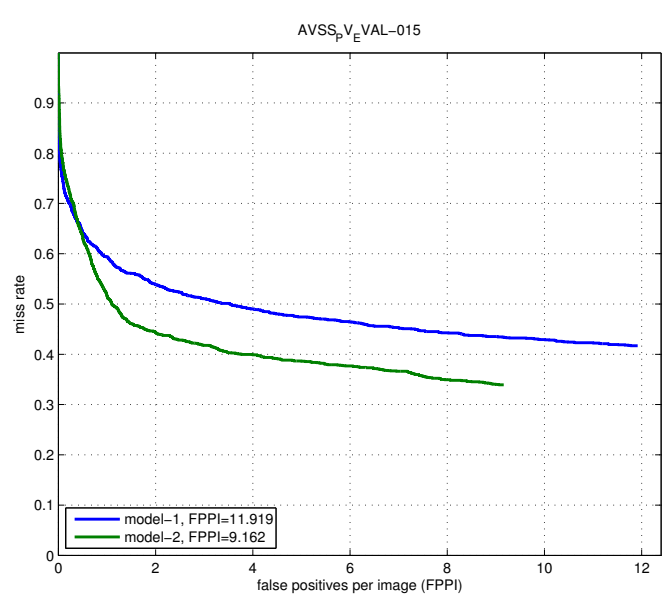

(d) Curva FPPI. Outros dois modelos AVSS $_{P} V_{E}$ VAL-015

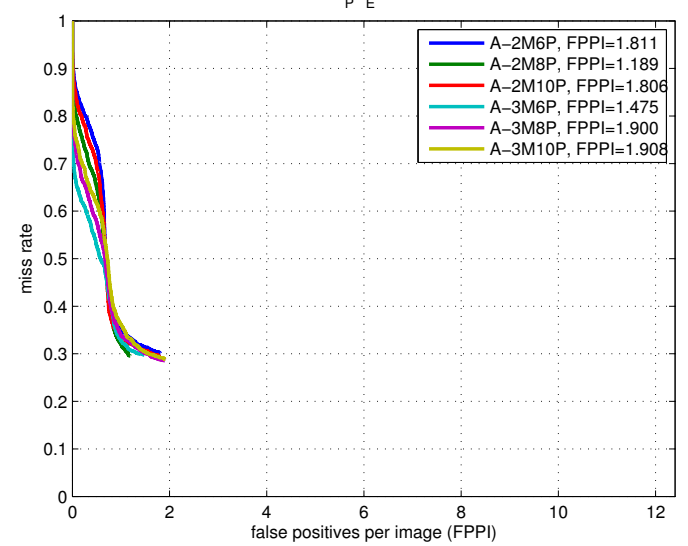

(f) Curva FPPI. MDPM Assimétrica

Figura 5.16: Curva precision vs. recall (PR) curva e curva miss rate vs. FPPI em AVSS_PV_EVAL015.avi. 


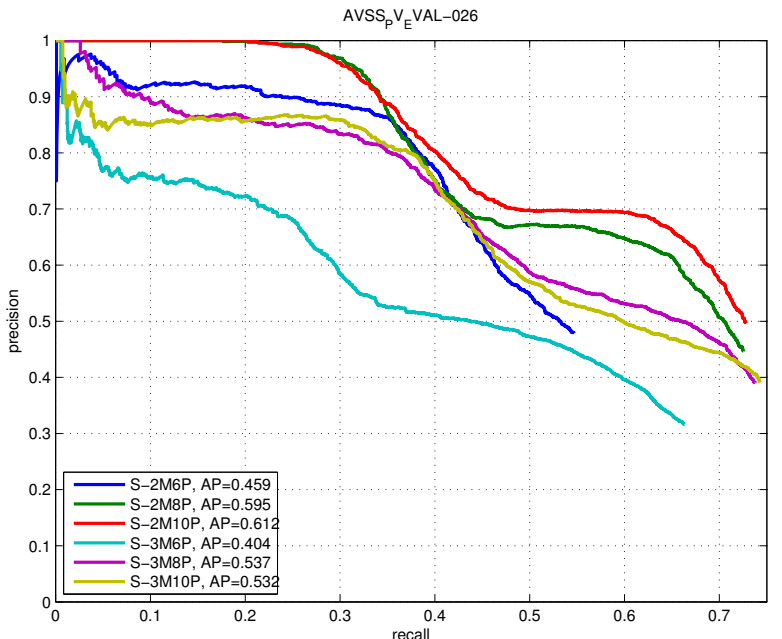

(a) Curva PR. MDPM Simétrica

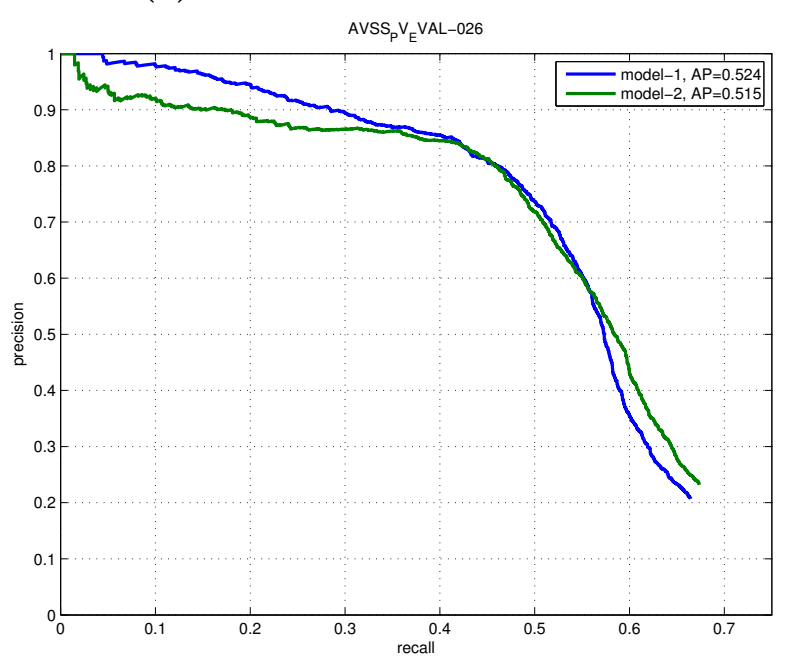

(c) Curva PR. Outros dois modelos

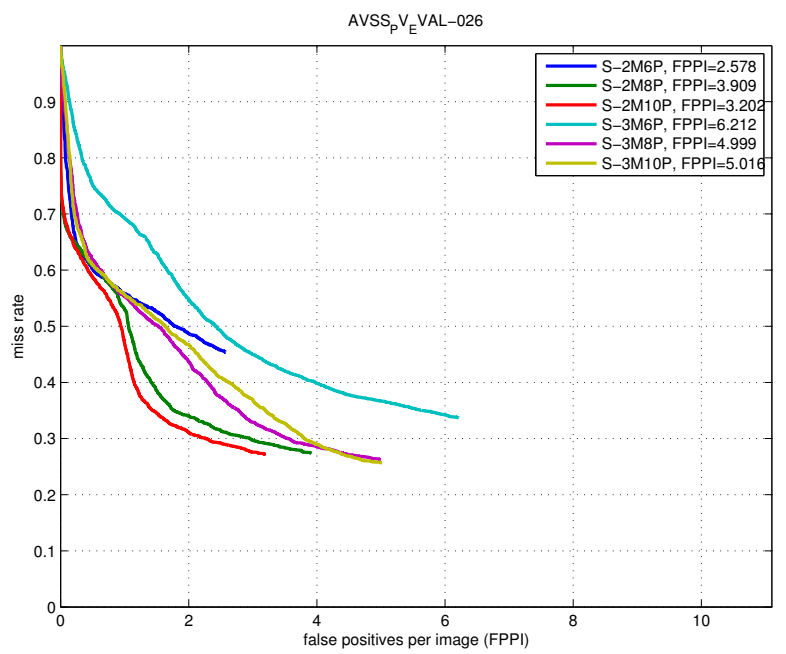

(e) Curva FPPI. MDPM Simétrica

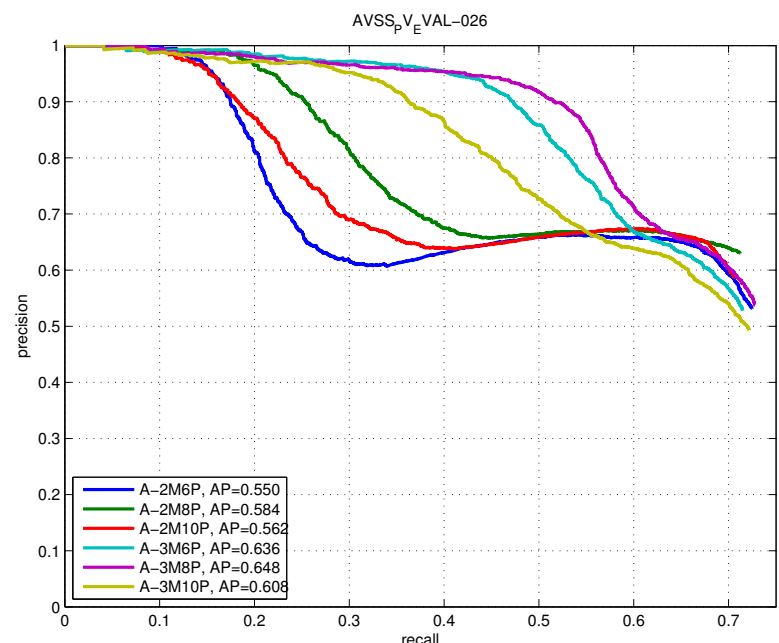

(b) Curva PR. MDPM Assimétrica

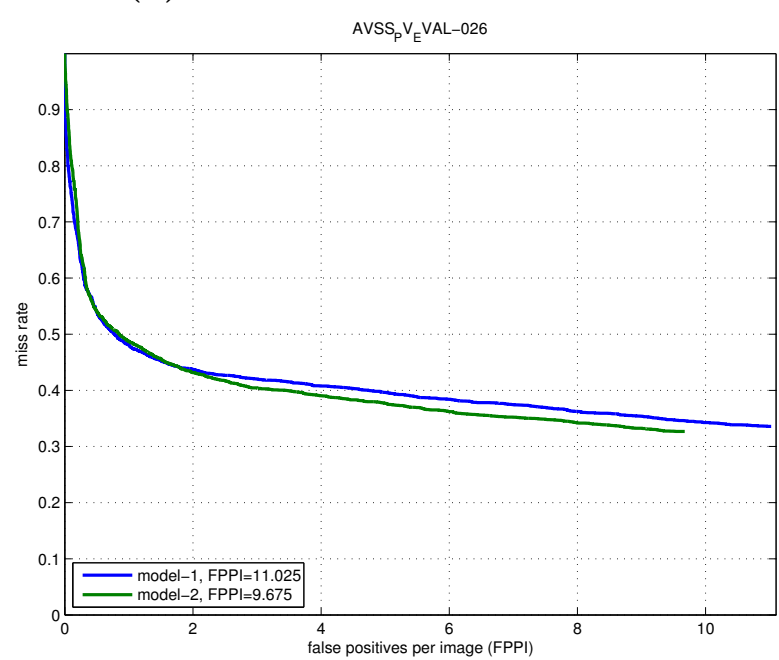

(d) Curva FPPI. Outros dois modelos

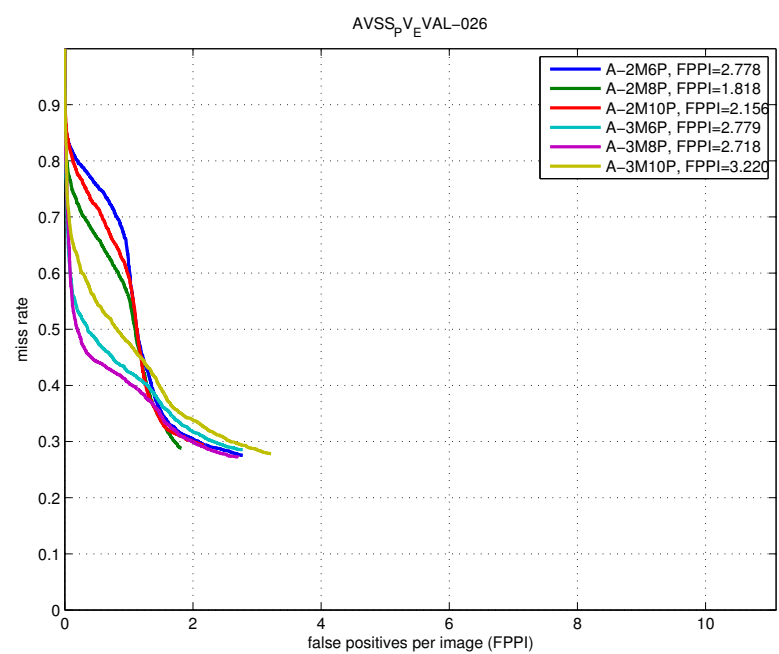

(f) Curva FPPI. MDPM Assimétrica

Figura 5.17: Curva precision vs. recall (PR) curva e curva miss rate vs. FPPI em AVSS_PV_EVAL026.avi. 


\subsection{Detecção de carros em vídeos obtidos por uma câmera dinâmica (E4)}

Neste experimento foi testado o método baseado em MDPM no conjunto descrito na Subseção 4.2.2 e sem considerar a informação do plano 3D .

Testamos usando MDPMs de dois e três componentes da mistura e seis, oito e dez partes do modelo. Adicionalmente, comparamos os nossos resultados obtidos com dois modelos de carro: model1 e model2 criados por Geiger et al. (2011) Desta forma, vamos poder verificar como que se comportam os modelos treinados com imagens específicas, neste caso de sequencias de vídeo obtidas por uma câmera dinâmica. Nossa hipótese é que os resultados serão melhores que no experimento anterior, dado que o treinamento e o teste consideram quadros de vídeos obtidos por uma câmera dinâmica.

A Tabela 5.7 mostra os valores (AP, recall, precision e FPPI) obtidos testando MDPMs simétricas e assimétricas. Na Tabela, os dois melhores resultados estão em negrito. O melhor AP foi obtido pela MDPM simétrica de 3 componentes e 8 partes, o menor número de FPPI por uma MDPM assimétrica de 2 componentes e 8 partes, o melhor recall por uma MDPM assimétrica de 3 componentes e 10 partes e a melhor precision por uma MDPM asimétrica de 2 componentes e 8 partes.

\begin{tabular}{l|cccc|cccc}
\hline & \multicolumn{6}{|c|}{ Simétrica } & \multicolumn{4}{c}{ Assimétrica } \\
MDPM & AP & Prec. & Recall & FPPI & AP & Prec. & Recall & FPPI \\
\hline $2 M 6 P$ & 0.154 & 0.412 & 0.244 & 0.936 & 0.135 & $\mathbf{0 . 4 5 6}$ & 0.267 & 0.856 \\
$2 M 8 P$ & 0.194 & 0.427 & 0.293 & 1.054 & 0.148 & $\mathbf{0 . 4 6 2}$ & 0.262 & $\mathbf{0 . 8 2 1}$ \\
$2 M 10 P$ & 0.181 & 0.454 & 0.298 & 0.960 & 0.139 & 0.455 & 0.262 & $\mathbf{0 . 8 4 5}$ \\
$3 M 6 P$ & 0.183 & 0.393 & 0.274 & 1.139 & 0.189 & 0.421 & 0.326 & 1.205 \\
$3 M 8 P$ & $\mathbf{0 . 2 0 4}$ & 0.443 & 0.302 & 1.022 & 0.191 & 0.413 & $\mathbf{0 . 3 3 0}$ & 1.259 \\
$3 M 10 P$ & $\mathbf{0 . 1 9 8}$ & 0.429 & 0.305 & 1.091 & 0.192 & 0.424 & $\mathbf{0 . 3 3 7}$ & 1.229 \\
\hline model 1 & 0.287 & 0.272 & 0.435 & 3.127 & - & - & - & - \\
model 2 & 0.280 & 0.289 & 0.429 & 2.824 & - & - & - & -
\end{tabular}

Tabela 5.7: AP, precision, recall e FPPI usando MDPMs simétricas e assimétricas, e os modelos de Geiger et al. (2011), avaliados no vídeo Leuven.

Para facilitar a análise dos resultados, os gráficos encontram-se normalizados nos eixos. A Figura 5.18 mostra a curva precision-recall e a curva miss rate-FPPI para as MDPMs simétricas e assimétricas e os outros modelos de Geiger et al. (2011) usados. Como pode-se observar dos gráficos, os resultados alcançam um nível de 33.7\% de recall e 1.23 de FPPI. Em Leibe et al. (2006), os autores reportam um recall de $50 \%$ e 1.3 em FPPI nesse nível de recall usando informação do ground plane. Em Cornelis et al. (2008), os autores reportam 4 FPPI e $50 \%$ de recall sem considerar o ground plane.

As curvas precision-recall e miss rate-FPPI para os modelos de Geiger et al. (2011) foram obtidas considerando um limiar de -0.5. Isso porque o limiar do modelo é muito maior e várias das detecções são descartadas. Usando estes modelos, uma precision ruim foi obtida em relação à obtida por nossas MDPMs. Por outro lado, como era esperado, pelo tipo de imagem usada no treinamento, um alto recall e AP é possível, tal como é mostrado na figura. Contudo, o número de FPPI obtido para cada modelo é maior que o obtido por nossas MDPMs. O melhor desses outros dois modelos tem uma taxa de FPPI de 2.8 com um recall de $42.9 \%$.

Na Figura 5.19 mostram-se os resultados obtidos para os quadros: 2, 47, 70, 123, 170, 202, $277,344,374,423,471,564,598,653,710,785,838,878,928,990,1040,1072,1106,1157$ e 1175. Os bounding boxes vermelhos foram obtidos pelo sistema com uma MDPM assimétrica de três componentes e oito partes, os bounding boxes verdes foram obtidos usando o model 2 de Geiger et al. (2011) e os bounding boxes azuis são os dados pelo conjunto de dados. 


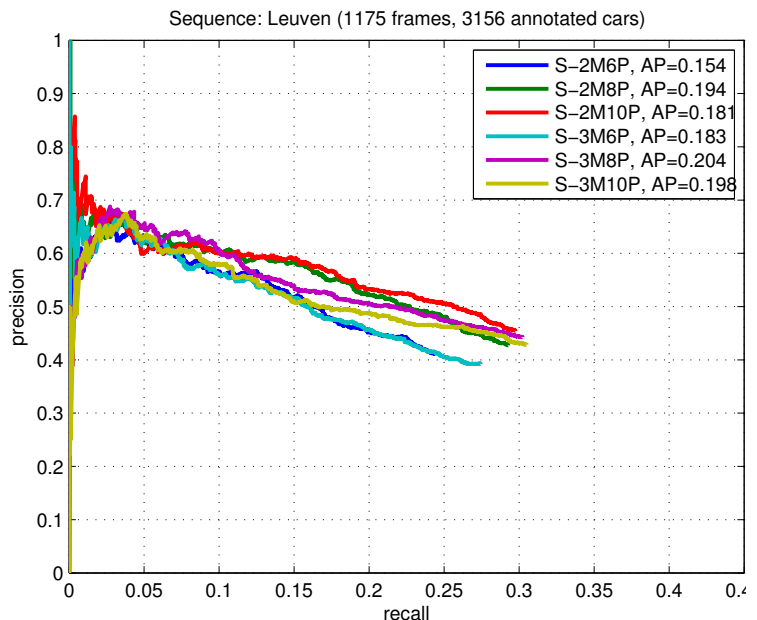

(a) Curva PR. MDPM Simétrica

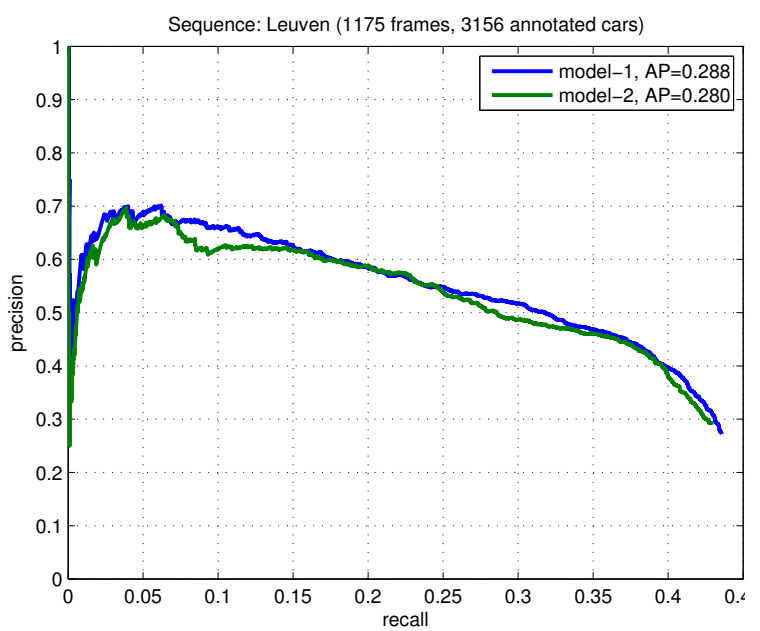

(c) Curva PR. Outros dois modelos

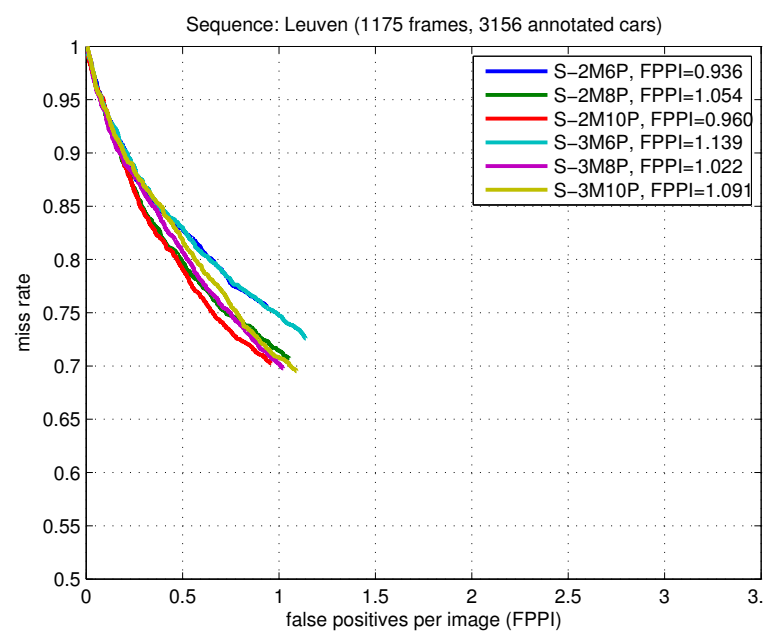

(e) Curva FPPI. MDPM Simétrica

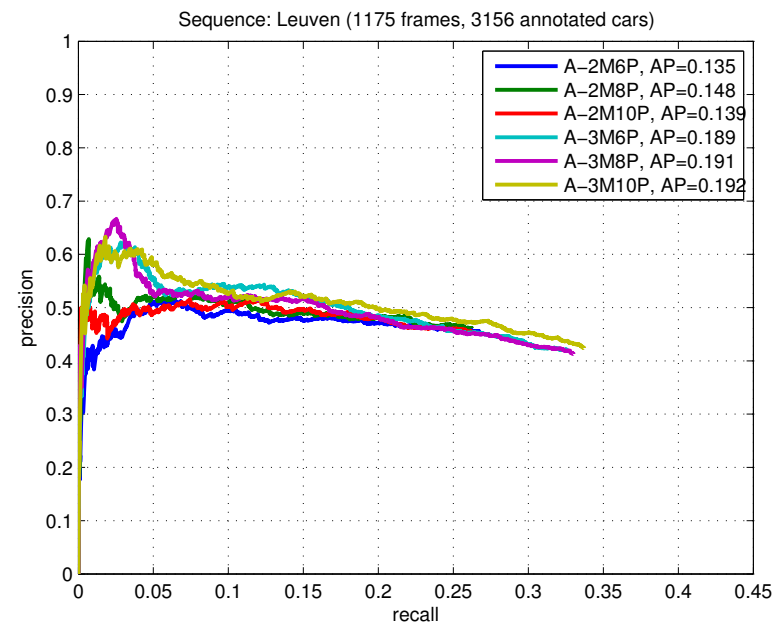

(b) Curva PR. MDPM Assimétrica

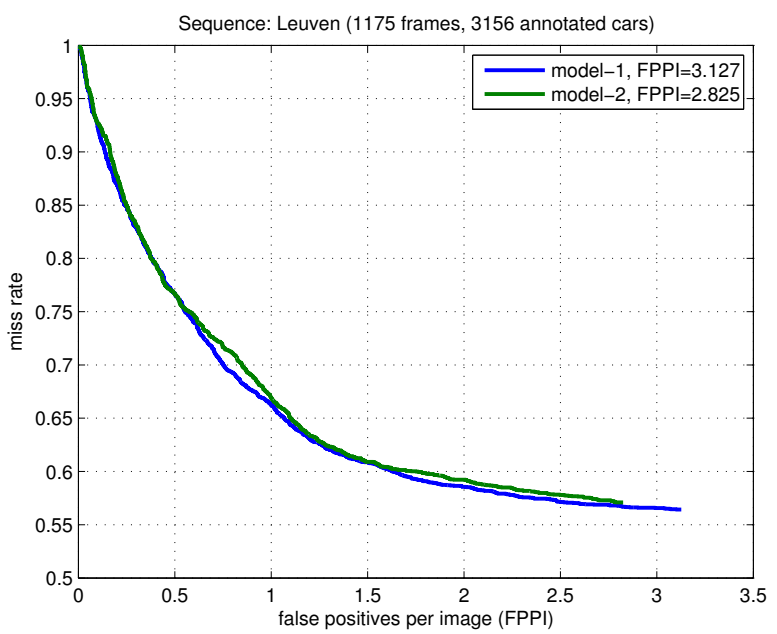

(d) Curva FPPI. Outros dois modelos

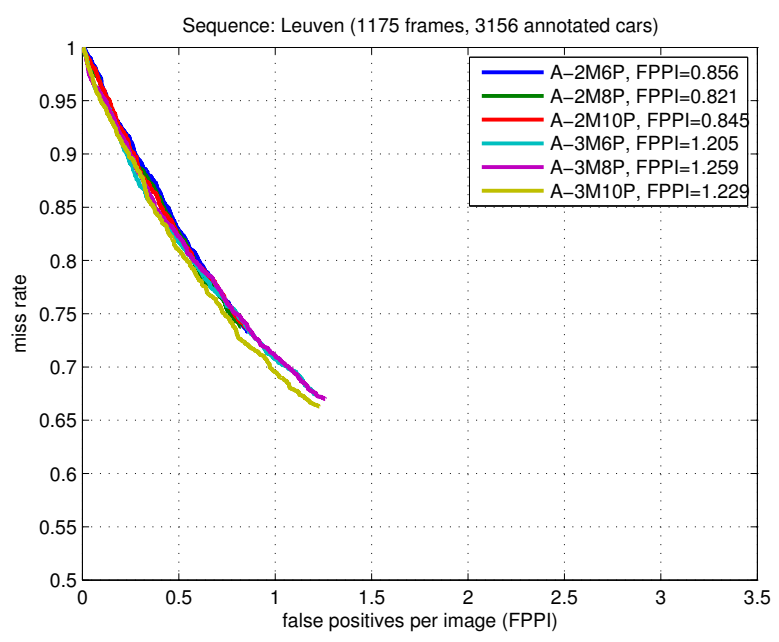

(f) Curva FPPI. MDPM Assimétrica

Figura 5.18: Curva precision vs. recall (PR) e curva miss rate vs. falso positivo por imagem (FPPI) no vídeo Leuven. 
Existem dois tipos de falsos positivos: as janelas de prédios consideradas como carro pelo sistema e os carros pequenos ou que encontram-se a uma distância maior à usada na anotação do vídeo. Este último caso reflete-se na última coluna da segunda linha de imagens da Figura 5.19. Achamos que, considerando a informação do plano 3D, melhores resultados podem ser obtidos.

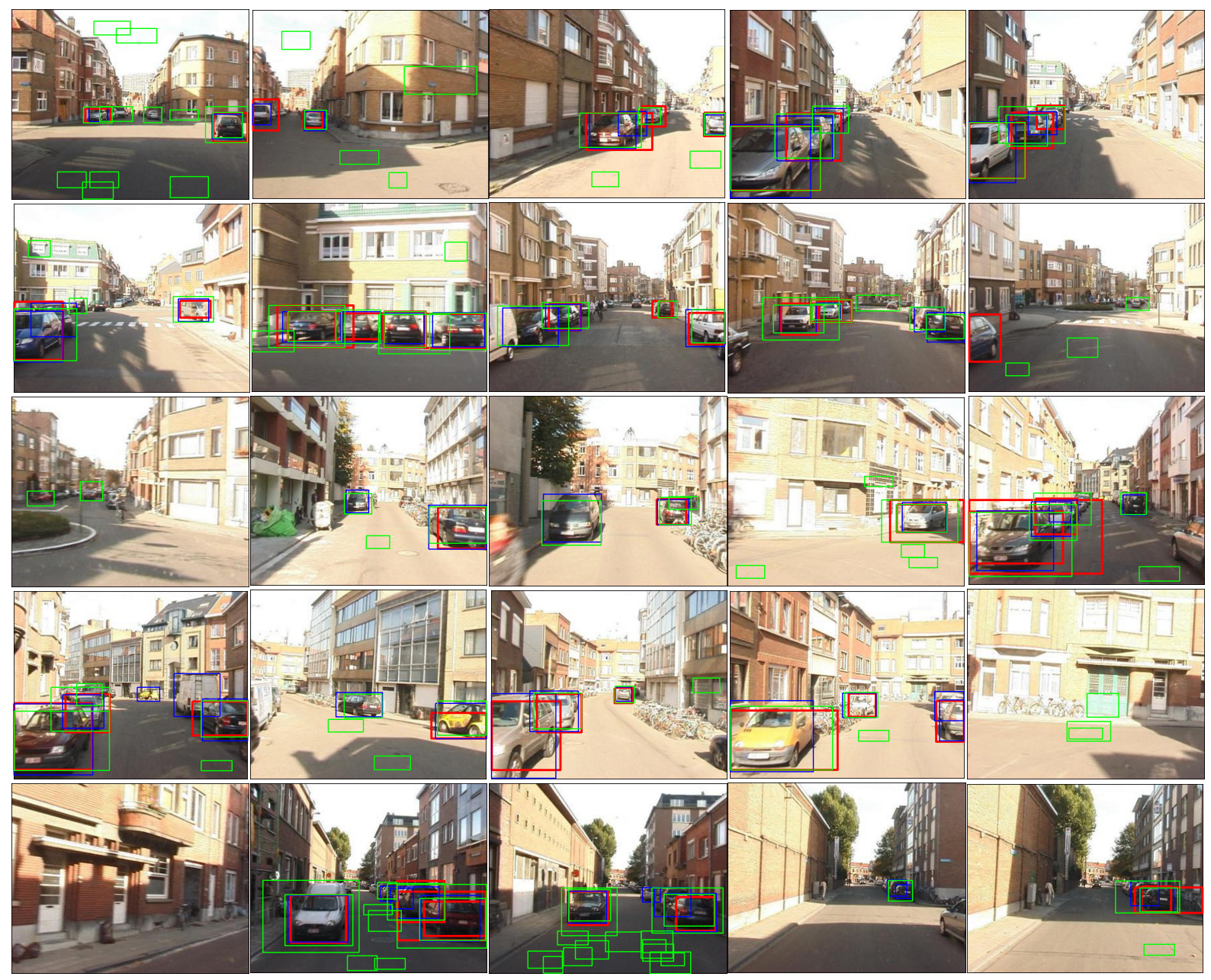

Figura 5.19: Resultados usando uma MDPM assimétrica de três componentes e oito partes e o model2 no vídeo Leuven. 


\subsection{Detecção de pessoas em vídeos (E5)}

O conjunto de dados usado é o conjunto de vídeos construído por García-Martín et al. (2012), o qual é descrito na Subseção 4.2.3.

Baseados nos resultados obtidos para a classe pessoa, apresentados na Tabela 5.1, foram selecionadas MDPMs de três componentes da mistura e seis, oito e dez partes do modelo para serem usadas neste experimento. Por outro lado, os vídeos foram obtidos por uma câmera estacionária. Neste caso, não definiu-se uma região de deteç̧ão, dado que os objetos variam de localização e na maioria dos casos aparecem ocupando a maior parte do quadro do vídeo. Os resultados obtidos são apresentados na Subseção 5.6.1.

Adicionalmente, foi feito um experimento unindo MDPMs simétricas e assimétricas e os resultados são descritos na Subseção 5.6.2.

\subsubsection{Resultados}

Os valores de precision, recall, AP e a pontuação $\mathrm{F}$ obtidos para cada um dos vídeos por categorias são mostrados nas Tabelas 5.8, 5.9, 5.10, 5.11 e 5.12 .

\begin{tabular}{l|l|cccc|cccc}
\hline \multirow{2}{*}{ Vídeo } & & \multicolumn{5}{|c|}{ Simétrica } & \multicolumn{4}{|c}{ Assimétrica } \\
& MDPM's & Prec. & Recall & AP & F & Prec. & Recall & AP & F \\
\hline \multirow{3}{*}{$V 11$} & 3M 6P & 0.270 & 0.840 & $\mathbf{0 . 6 7 5}$ & 0.408 & 0.587 & 0.538 & 0.397 & 0.561 \\
& $\mathbf{3 M ~ 8 P ~}$ & 0.373 & 0.916 & 0.611 & 0.530 & $\mathbf{0 . 6 1 1}$ & 0.580 & 0.413 & $\mathbf{0 . 5 9 5}$ \\
& 3M 10P & 0.295 & $\mathbf{0 . 9 3 3}$ & 0.543 & 0.448 & 0.492 & 0.521 & 0.427 & 0.506 \\
\hline \multirow{3}{*}{$V 12$} & 3M 6P & 0.324 & $\mathbf{0 . 9 8 5}$ & $\mathbf{0 . 9 0 6}$ & 0.487 & $\mathbf{0 . 7 2 1}$ & $\mathbf{0 . 9 8 5}$ & 0.812 & $\mathbf{0 . 8 3 3}$ \\
& 3M 8P & 0.289 & $\mathbf{0 . 9 8 5}$ & 0.738 & 0.447 & 0.670 & 0.970 & 0.873 & 0.793 \\
& 3M 10P & 0.312 & 0.978 & 0.770 & 0.473 & 0.663 & 0.985 & 0.868 & 0.793 \\
\hline \multirow{3}{*}{$V 13$} & 3M 6P & 0.297 & 0.948 & 0.837 & 0.452 & 0.918 & 0.918 & $\mathbf{0 . 8 9 9}$ & 0.918 \\
& 3M 8P & 0.389 & $\mathbf{0 . 9 5 9}$ & 0.863 & 0.554 & $\mathbf{0 . 9 2 9}$ & 0.938 & 0.896 & $\mathbf{0 . 9 3 3}$ \\
& 3M 10P & 0.330 & $\mathbf{0 . 9 5 9}$ & 0.898 & 0.491 & 0.818 & 0.928 & 0.877 & 0.870 \\
\hline \multirow{3}{*}{$V 14$} & 3M 6P & 0.299 & 0.977 & 0.849 & 0.458 & $\mathbf{0 . 7 8 5}$ & 0.945 & 0.875 & $\mathbf{0 . 8 5 8}$ \\
& 3M 8P & 0.335 & $\mathbf{0 . 9 8 4}$ & 0.863 & 0.499 & 0.775 & 0.947 & $\mathbf{0 . 8 8 5}$ & 0.853 \\
& 3M 10P & 0.295 & 0.977 & 0.852 & 0.453 & 0.740 & 0.947 & 0.878 & 0.831 \\
\hline
\end{tabular}

Tabela 5.8: Precision, recall, AP e a pontuação $F$ obtidos para a categoria 1, mudando os parâmetros das MDPMs simétricas e assimétricas.

Na Tabela 5.8, observa-se um alto valor de recall usando MDPMs simétricas. Porém, em relação à precision, $\mathrm{AP}$ e pontuação $\mathrm{F}$, observamos melhores resultados usando MDPMs assimétricas. Nas Tabelas 5.9 e 5.12, tem-se um valor de recall e AP maior usando MDPMs simétricas, e uma precision e pontuação F maior usando MDPMs assimétricas. Nas Tabelas 5.10 e 5.11, tem-se um valor de recall maior usando MDPMs simétricas e nas outras métricas com as MDPMs assimétricas.

Em geral, observa-se um alto valor de recall considerando MDPMs simétricas e uma alta precision considerando MDPMs assimétricas. O valor de recall é muito próximo para MDPMs simétricas e assimétricas, enquanto que a precision das MDPMs assimétricas chega a ser o dobro, as vezes até três vezes mais, do obtido pelas MDPMs simétricas. Os altos valores de precision acarretam que as pontuações F sejam melhores considerando MDPMs assimétricas. Enquanto que para os valores de AP pode-se dizer que são quase são muito próximas, tanto para MDPMs simétricas como assimétricas. Em conclusão, os melhores resultados foram obtidos por MDPMs assimétricas. Com respeito ao número de partes do modelo, na maioria das medidas têm-se valores altos usando modelos de 8 e 6 partes.

Comparando alguns dos resultados obtidos para o valor de recall, com os de García-Martín et al. (2011), temos que para os vídeos $V 11, V 12$ os resultados são equivalentes. Para os vídeos $V 21, V 22$ 


\begin{tabular}{|c|c|c|c|c|c|c|c|c|c|}
\hline \multirow[b]{2}{*}{ Vídeo } & \multirow[b]{2}{*}{ MDPM's } & \multicolumn{4}{|c|}{ Simétrica } & \multicolumn{4}{|c|}{ Assimétrica } \\
\hline & & Prec. & Recall & $\mathbf{A P}$ & $\mathbf{F}$ & Prec. & Recall & $\mathbf{A P}$ & $\mathbf{F}$ \\
\hline \multirow{3}{*}{$V 21$} & $3 \mathrm{M} 6 \mathrm{P}$ & 0.277 & 0.997 & 0.606 & 0.434 & 0.721 & 0.988 & 0.849 & 0.834 \\
\hline & $3 \mathrm{M} 8 \mathbf{P}$ & 0.321 & 0.997 & 0.551 & 0.486 & 0.757 & 0.994 & 0.873 & 0.859 \\
\hline & $3 \mathrm{M} 10 \mathrm{P}$ & 0.302 & 0.994 & 0.616 & 0.464 & 0.722 & 0.991 & 0.882 & 0.835 \\
\hline \multirow{3}{*}{$V 22$} & $3 \mathrm{M} 6 \mathrm{P}$ & 0.268 & 0.967 & 0.799 & 0.420 & 0.677 & 0.837 & 0.608 & 0.749 \\
\hline & $3 \mathrm{M} 8 \mathrm{P}$ & 0.341 & 0.980 & 0.664 & 0.506 & 0.740 & 0.837 & 0.693 & 0.785 \\
\hline & $3 \mathrm{M} 10 \mathrm{P}$ & 0.315 & 0.987 & 0.764 & 0.478 & 0.722 & 0.830 & 0.736 & 0.772 \\
\hline \multirow{3}{*}{$V 23$} & $3 \mathrm{M} 6 \mathrm{P}$ & 0.332 & 0.886 & 0.685 & 0.483 & 0.732 & 0.761 & 0.645 & 0.746 \\
\hline & $3 \mathrm{M} 8 \mathrm{P}$ & 0.371 & 0.866 & 0.665 & 0.519 & 0.700 & 0.756 & 0.644 & 0.727 \\
\hline & $3 \mathrm{M} \mathrm{10P}$ & 0.338 & 0.847 & 0.678 & 0.483 & 0.628 & 0.711 & 0.627 & 0.667 \\
\hline \multirow{3}{*}{$V 24$} & $3 \mathrm{M} 6 \mathrm{P}$ & 0.403 & 0.900 & 0.702 & 0.557 & 0.667 & 0.622 & 0.578 & 0.644 \\
\hline & $3 \mathrm{M} \mathrm{8P}$ & 0.417 & 0.922 & 0.713 & 0.574 & 0.602 & 0.622 & 0.573 & 0.612 \\
\hline & $3 \mathrm{M} 10 \mathrm{P}$ & 0.484 & 0.822 & 0.569 & 0.609 & 0.592 & 0.644 & 0.572 & 0.617 \\
\hline \multirow{3}{*}{$V 25$} & $3 \mathrm{M} 6 \mathrm{P}$ & 0.324 & 0.977 & 0.876 & 0.486 & 0.608 & 0.948 & 0.884 & 0.741 \\
\hline & $3 \mathrm{M} 8 \mathrm{P}$ & 0.357 & 0.979 & 0.866 & 0.524 & 0.592 & 0.948 & 0.889 & 0.729 \\
\hline & $3 \mathrm{M} 10 \mathrm{P}$ & 0.455 & 0.977 & 0.897 & 0.620 & 0.572 & 0.943 & 0.887 & 0.712 \\
\hline
\end{tabular}

Tabela 5.9: Precision, recall, AP e a pontuação F obtidos para a categoria 2, mudando os parâmetros das MDPMs simétricas e assimétricas.

\begin{tabular}{l|l|cccc|cccc}
\hline \multirow{2}{*}{ Vídeo } & \multirow{2}{*}{ MDPM's } & Prec. & Recall & AP & F & Prec. & Recall & AP & F \\
\hline \multirow{3}{*}{$V 31$} & 3M 6P & 0.519 & $\mathbf{0 . 7 9 5}$ & 0.632 & 0.628 & 0.733 & 0.713 & 0.618 & 0.723 \\
& 3M 8P & 0.478 & 0.754 & 0.558 & 0.585 & $\mathbf{0 . 7 7 1}$ & 0.713 & 0.614 & $\mathbf{0 . 7 4 1}$ \\
& 3M 10P & 0.423 & 0.711 & 0.568 & 0.531 & 0.753 & 0.722 & $\mathbf{0 . 6 3 4}$ & 0.737 \\
\hline \multirow{3}{*}{$V 32$} & 3M 6P & 0.362 & 0.872 & 0.775 & 0.511 & $\mathbf{0 . 7 3 5}$ & 0.782 & 0.697 & $\mathbf{0 . 7 5 8}$ \\
& 3M 8P & 0.435 & $\mathbf{0 . 8 8 7}$ & $\mathbf{0 . 7 9 6}$ & 0.584 & 0.704 & 0.800 & 0.685 & 0.749 \\
& 3M 10P & 0.444 & 0.878 & 0.789 & 0.590 & 0.704 & 0.788 & 0.682 & 0.744 \\
\hline
\end{tabular}

Tabela 5.10: Precision, recall, AP e a pontuação F obtidos para a categoria 3, mudando os parâmetros das MDPMs simétricas e assimétricas.

temos melhores resultados. Eles alcançam um pouco mais do que $70 \%$, enquanto nossos resultados alcançam mais do que $97 \%$. Por outro lado, eles alcançaram melhores resultados com o vídeo $V 32$, com uma diferença de aproximadamente $10 \%$. 


\begin{tabular}{|c|c|c|c|c|c|c|c|c|c|}
\hline \multirow[b]{2}{*}{ Vídeo } & \multirow[b]{2}{*}{ MDPM's } & \multicolumn{4}{|c|}{ Simétrica } & \multicolumn{4}{|c|}{ Assimétrica } \\
\hline & & Prec. & Recall & AP & F & Prec. & Recall & $\mathbf{A P}$ & $\mathbf{F}$ \\
\hline \multirow{3}{*}{$V 41$} & $3 \mathrm{M} 6 \mathrm{P}$ & 0.285 & 0.925 & 0.792 & 0.436 & 0.612 & 0.840 & 0.749 & 0.708 \\
\hline & $3 \mathrm{M} 8 \mathrm{P}$ & 0.296 & 0.902 & 0.707 & 0.446 & 0.633 & 0.847 & 0.766 & 0.725 \\
\hline & $3 \mathrm{M} \mathrm{10P}$ & 0.250 & 0.885 & 0.645 & 0.389 & 0.584 & 0.850 & 0.766 & 0.693 \\
\hline \multirow{3}{*}{$V 42$} & $3 \mathrm{M} 6 \mathrm{P}$ & 0.237 & 0.936 & 0.732 & 0.378 & 0.610 & 0.788 & 0.584 & 0.688 \\
\hline & $3 \mathrm{M} 8 \mathrm{P}$ & 0.299 & 0.937 & 0.694 & 0.454 & 0.604 & 0.781 & 0.614 & 0.681 \\
\hline & $3 \mathrm{M} \mathrm{10P}$ & 0.257 & 0.928 & 0.724 & 0.403 & 0.591 & 0.768 & 0.620 & 0.668 \\
\hline \multirow{3}{*}{$V 43$} & $3 \mathrm{M} 6 \mathrm{P}$ & 0.251 & 0.876 & 0.648 & 0.390 & 0.684 & 0.637 & 0.519 & 0.660 \\
\hline & $3 \mathrm{M} 8 \mathrm{P}$ & 0.288 & 0.854 & 0.603 & 0.431 & 0.688 & 0.646 & 0.572 & 0.666 \\
\hline & $3 \mathrm{M} \mathrm{10P}$ & 0.225 & 0.776 & 0.533 & 0.349 & 0.647 & 0.597 & 0.495 & 0.621 \\
\hline \multirow{3}{*}{$V 44$} & $3 \mathrm{M} 6 \mathrm{P}$ & 0.298 & 0.932 & 0.784 & 0.451 & 0.618 & 0.801 & 0.651 & 0.697 \\
\hline & $3 \mathrm{M} 8 \mathrm{P}$ & 0.335 & 0.930 & 0.692 & 0.493 & 0.658 & 0.829 & 0.681 & 0.734 \\
\hline & $3 \mathrm{M} 10 \mathrm{P}$ & 0.265 & 0.896 & 0.635 & 0.409 & 0.592 & 0.811 & 0.655 & 0.685 \\
\hline \multirow{3}{*}{$V 45$} & $3 \mathrm{M} 6 \mathrm{P}$ & 0.335 & 0.942 & 0.715 & 0.494 & 0.648 & 0.838 & 0.703 & 0.731 \\
\hline & $3 \mathrm{M} 8 \mathrm{P}$ & 0.368 & 0.936 & 0.588 & 0.528 & 0.664 & 0.858 & 0.738 & 0.749 \\
\hline & $3 \mathrm{M} \mathrm{10P}$ & 0.330 & 0.928 & 0.656 & 0.487 & 0.642 & 0.856 & 0.740 & 0.734 \\
\hline
\end{tabular}

Tabela 5.11: Precision, recall, AP e a pontuação F obtidos para a categoria 4, mudando os parâmetros das MDPMs simétricas e assimétricas.

\begin{tabular}{l|l|cccc|cccc}
\hline & & \multicolumn{6}{|c}{ Simétrica } & \multicolumn{4}{|c}{ Assimétrica } \\
Vídeo & MDPM's & Prec. & Recall & AP & F & Prec. & Recall & AP & F \\
\hline \multirow{3}{*}{$V 51$} & 3M 6P & 0.328 & 0.956 & 0.711 & 0.488 & 0.599 & 0.802 & 0.693 & 0.686 \\
& 3M 8P & 0.338 & $\mathbf{0 . 9 5 9}$ & 0.695 & 0.500 & $\mathbf{0 . 6 4 9}$ & 0.812 & $\mathbf{0 . 7 2 7}$ & $\mathbf{0 . 7 2 1}$ \\
& 3M 10P & 0.301 & 0.952 & 0.644 & 0.458 & 0.604 & 0.803 & 0.715 & 0.690 \\
\hline \multirow{3}{*}{$V 52$} & 3M 6P & 0.184 & 0.797 & 0.582 & 0.299 & $\mathbf{0 . 6 2 8}$ & 0.554 & 0.473 & $\mathbf{0 . 5 8 9}$ \\
& 3M 8P & 0.223 & $\mathbf{0 . 8 3 1}$ & $\mathbf{0 . 6 0 3}$ & 0.352 & 0.474 & 0.549 & 0.476 & 0.509 \\
& 3M 10P & 0.173 & 0.615 & 0.396 & 0.270 & 0.452 & 0.527 & 0.446 & 0.487 \\
\hline \multirow{3}{*}{$V 53$} & 3M 6P & 0.221 & 0.900 & 0.665 & 0.354 & $\mathbf{0 . 6 2 9}$ & 0.834 & 0.663 & $\mathbf{0 . 7 1 7}$ \\
& 3M 8P & 0.233 & $\mathbf{0 . 9 1 0}$ & $\mathbf{0 . 7 0 6}$ & 0.371 & 0.580 & 0.848 & 0.658 & 0.689 \\
& 3M 10P & 0.180 & 0.824 & 0.644 & 0.296 & 0.538 & 0.836 & 0.684 & 0.654 \\
\hline \multirow{4}{*}{$V 54$} & 3M 6P & 0.134 & $\mathbf{0 . 8 9 0}$ & $\mathbf{0 . 6 4 0}$ & 0.234 & $\mathbf{0 . 4 0 1}$ & 0.635 & 0.426 & $\mathbf{0 . 4 9 2}$ \\
& 3M 8P & 0.143 & $\mathbf{0 . 8 9 0}$ & 0.544 & 0.246 & 0.316 & 0.585 & 0.396 & 0.410 \\
& 3M 10P & 0.156 & 0.851 & 0.606 & 0.263 & 0.276 & 0.596 & 0.398 & 0.378 \\
\hline \multirow{4}{*}{$V 55$} & 3M 6P & 0.269 & $\mathbf{0 . 9 0 9}$ & 0.718 & 0.415 & 0.621 & 0.853 & 0.712 & 0.719 \\
& 3M 8P & 0.271 & 0.894 & 0.530 & 0.416 & $\mathbf{0 . 6 2 8}$ & 0.862 & $\mathbf{0 . 7 4 9}$ & $\mathbf{0 . 7 2 6}$ \\
& 3M 10P & 0.296 & 0.873 & 0.646 & 0.442 & 0.591 & 0.860 & $\mathbf{0 . 7 4 9}$ & 0.700 \\
\hline
\end{tabular}

Tabela 5.12: Precision, recall, AP e a pontuação F obtidos para a categoria 5, mudando os parâmetros das MDPMs simétricas e assimétricas. 
Adicionalmente, nas Figuras 5.20, 5.21, 5.22, 5.23, 5.24, 5.25, 5.26, 5.27, 5.28, 5.29, 5.30, 5.31, 5.32 e 5.33 apresenta-se as curvas de Precision vs. Recall e alguns exemplos de detecções usando MDPMs assimétricas de 3 componentes e 8 partes. Os exemplos pertencem aos quadros: 1, 16, 31, 46, 61, 76, 91, 106, 121, 136, 151, 166, 181, 196, 211, 226, 241, 256, 271, 286 dos vídeos.

Observamos que os falsos positivos referem para o caso de múltiplas detecções, e alguns falsos negativos quando a pessoa aparece de lado e inclina-se para pegar ou deixar algum objeto no chão, quando uma pessoa esta sentada de lado com a perna dobrada e o fundo complexo. Adicionalmente, percebemos que no caso de vídeos com a mesma ação das pessoas mas com fundo diferente, a complexidade do fundo influi negativamente nos resultados, dado que com maior complexidade do fundo tem-se maior quantidade de falsos positivos. Por exemplo, no vídeo $V 55$ foram detectados em alguns quadros como verdadeiro positivos uma pequena árvore que aparece se mexendo, coisa que não aparece no vídeo $V 45$ e portanto não se tem esses falsos positivos.
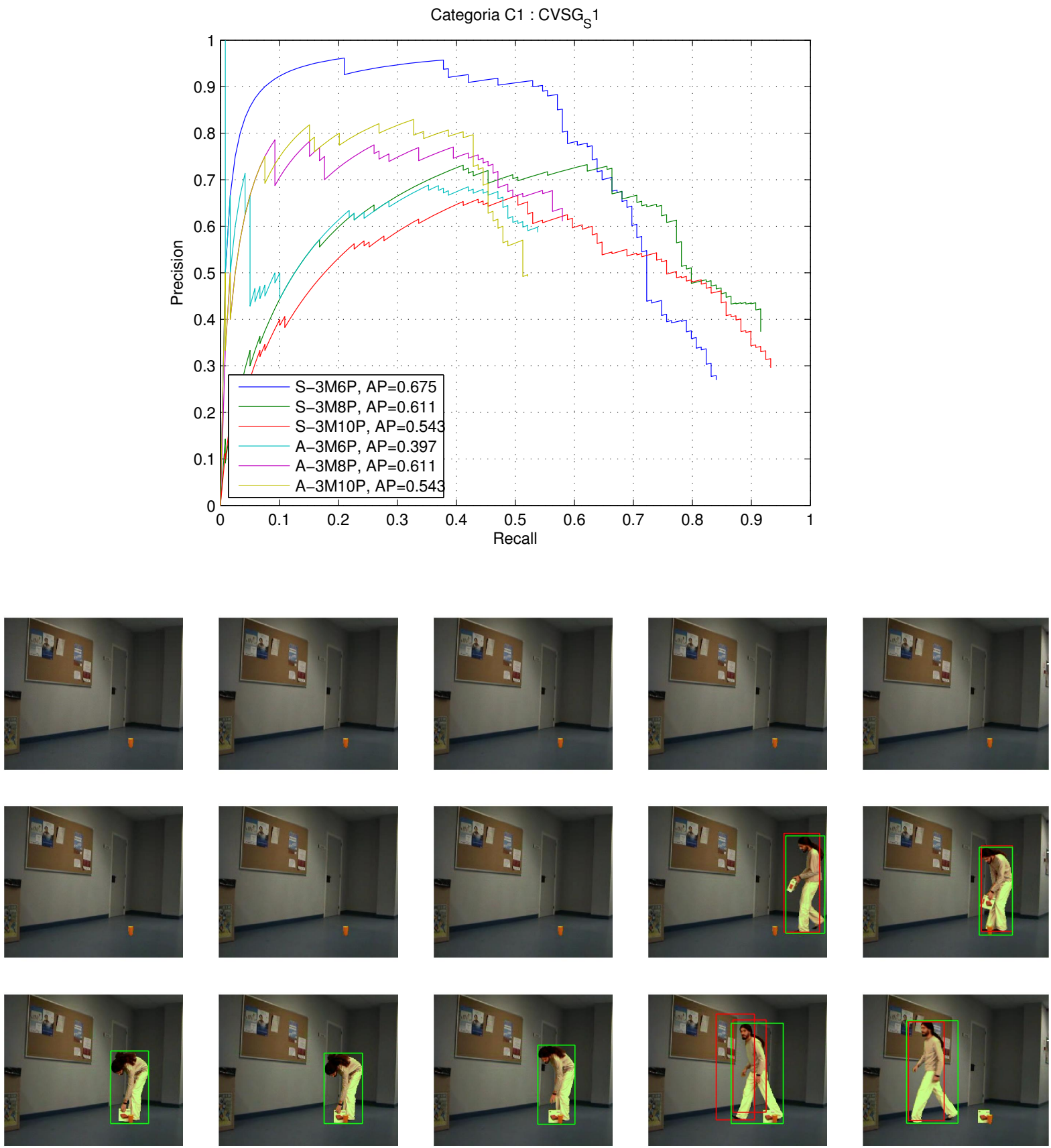

Figura 5.20: Curva de Precision vs. Recall e o AP para o vídeo V11, usando MDPMs simétricas e assimétricas de 3 componentes e 6,8 e 10 partes. 

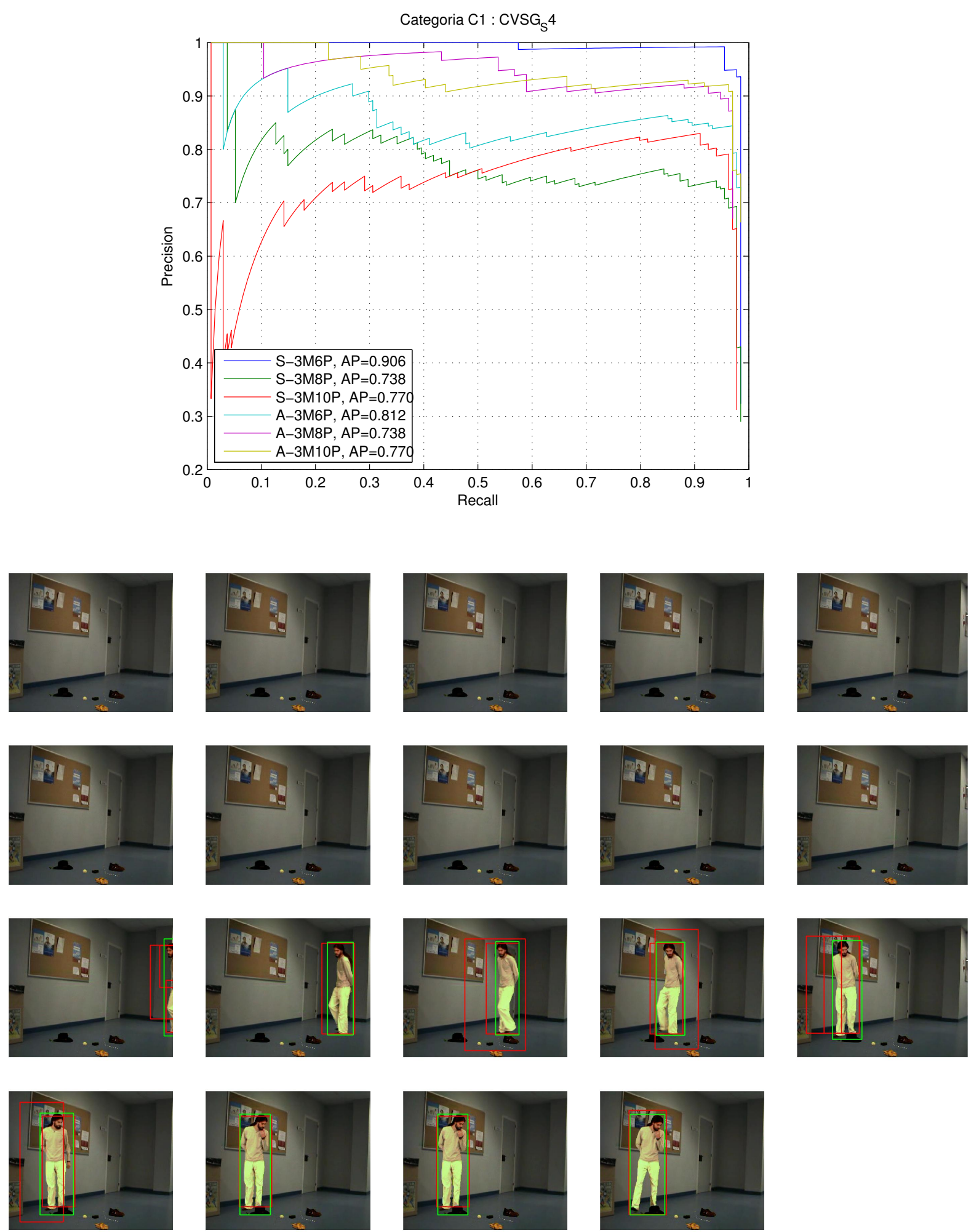

Figura 5.21: Curva de Precision vs. Recall e o AP para o vídeo V12, usando MDPMs simétricas e assimétricas de 3 componentes e 6,8 e 10 partes. 

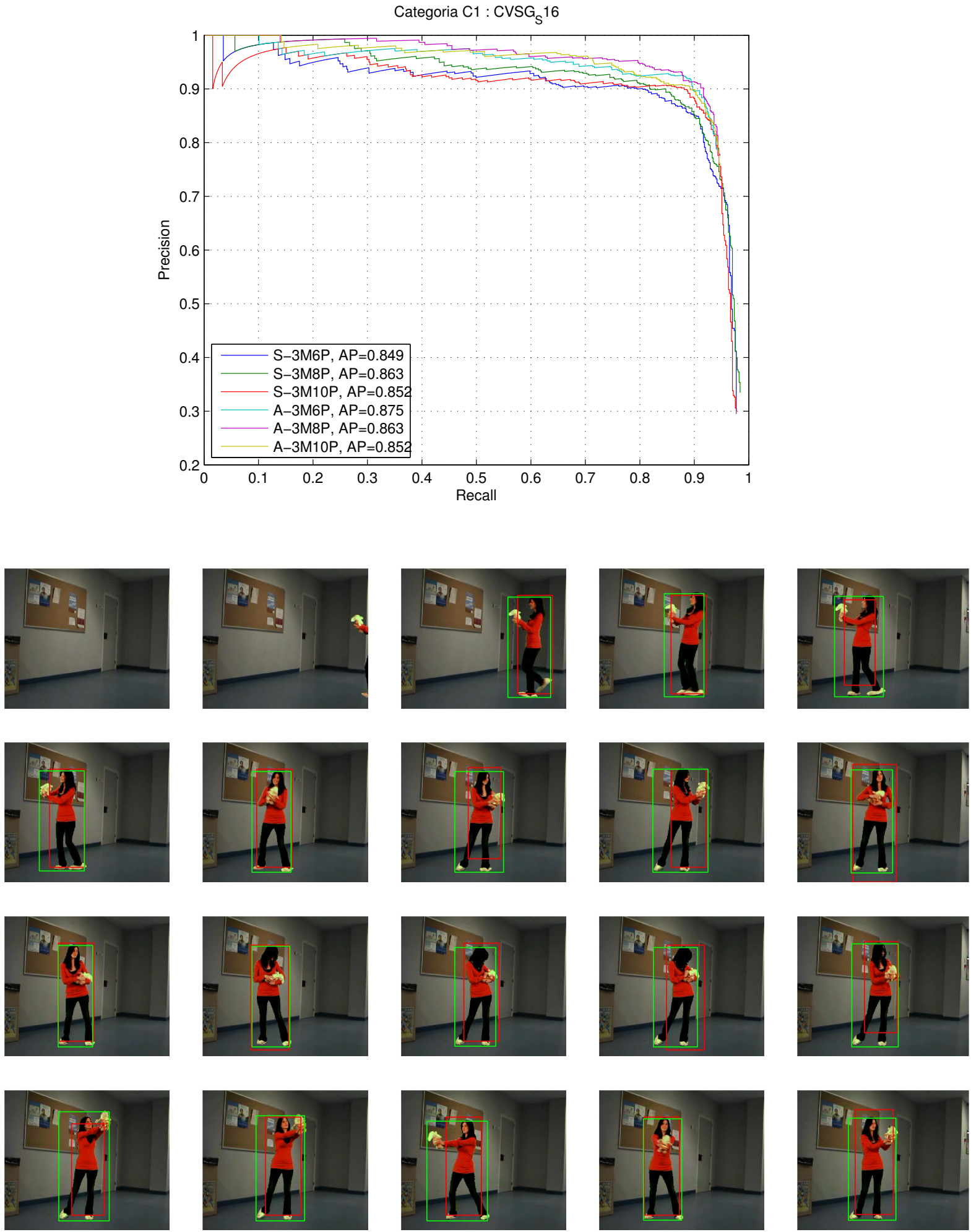

Figura 5.22: Curva de Precision vs. Recall e o AP para o vídeo V14, usando MDPMs simétricas e assimétricas de 3 componentes e 6,8 e 10 partes. 

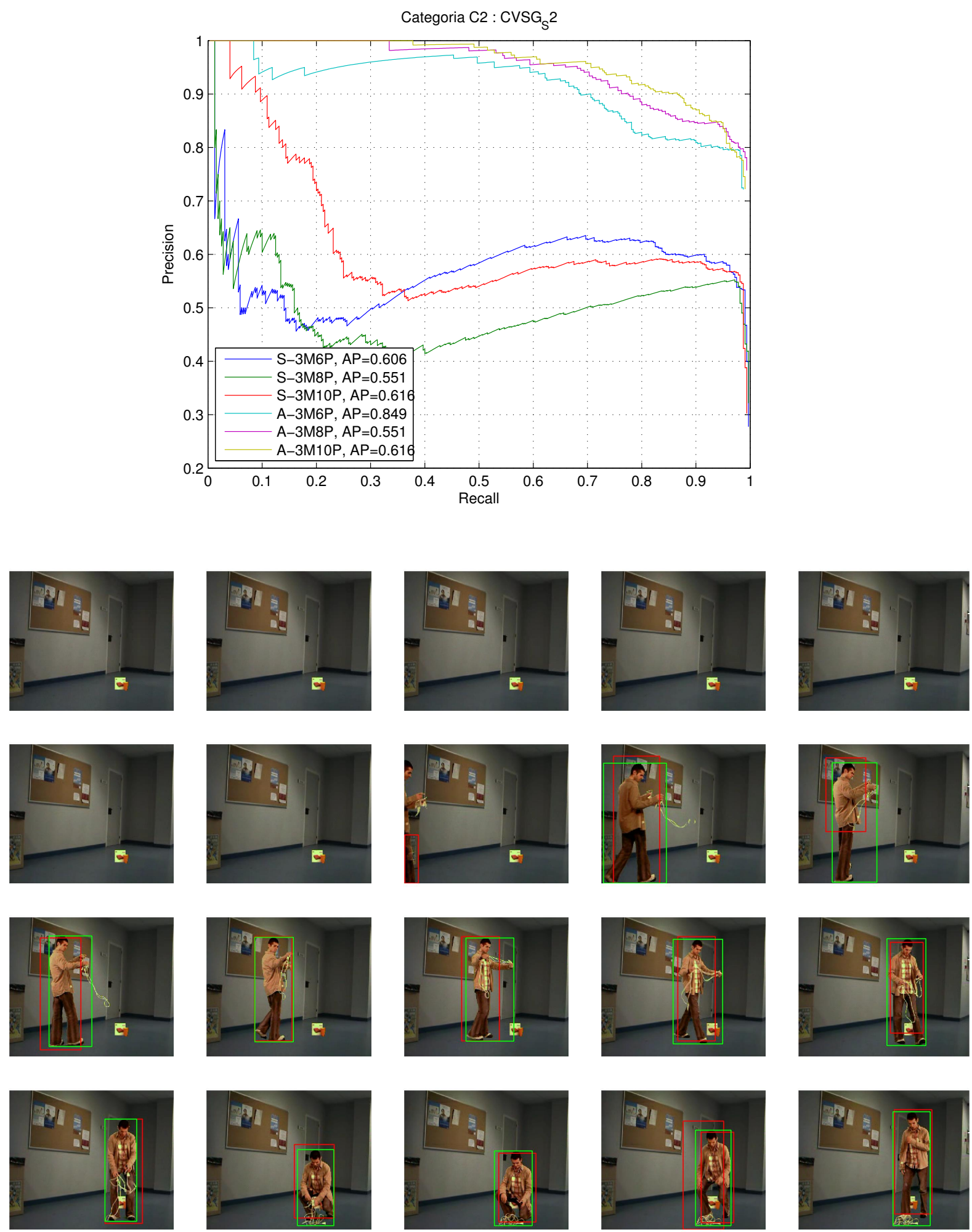

Figura 5.23: Curva de Precision vs. Recall e o AP para o vídeo V21, usando MDPMs simétricas e assimétricas de 3 componentes e 6,8 e 10 partes. 

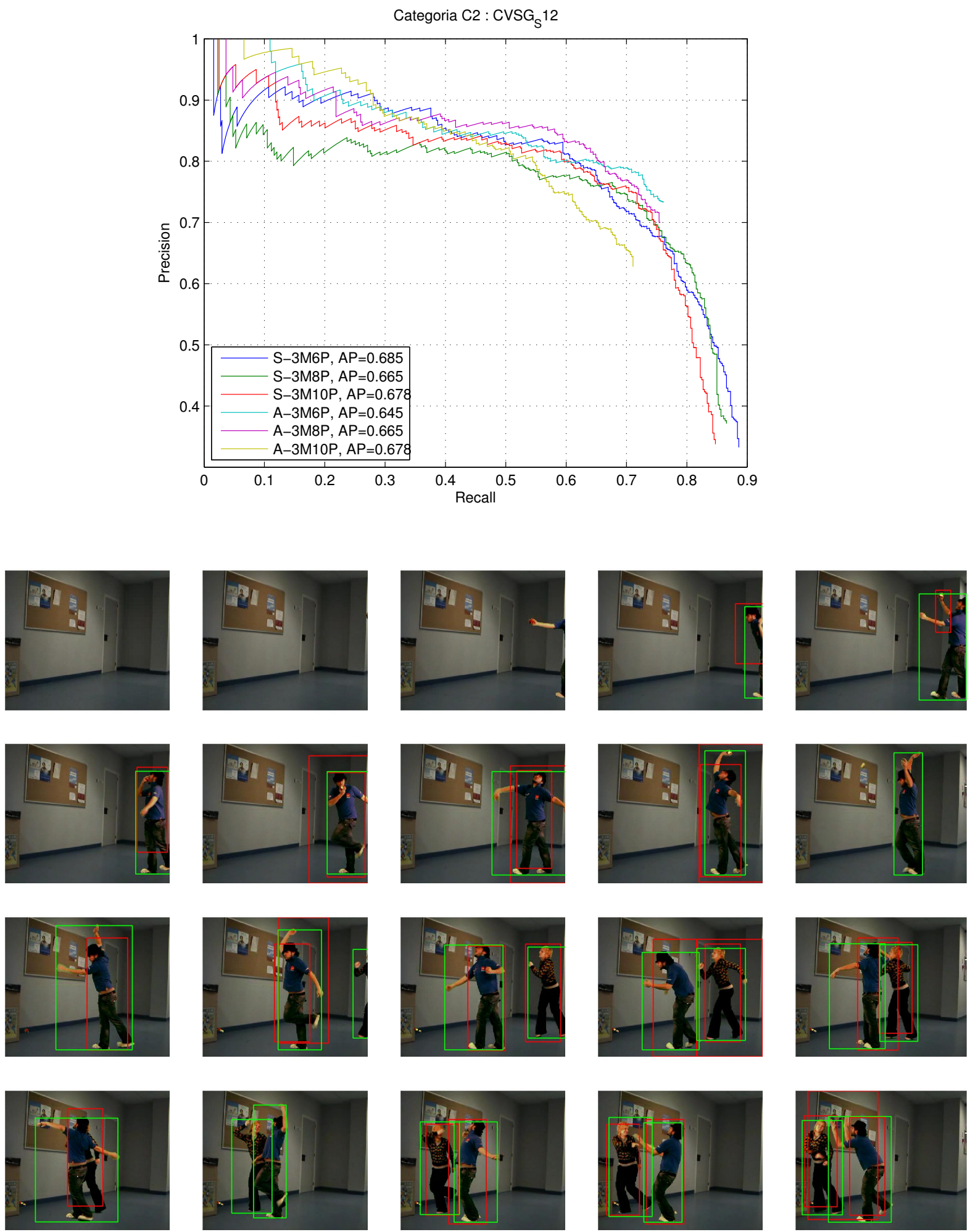

Figura 5.24: Curva de Precision vs. Recall e o AP para o vídeo V23, usando MDPMs simétricas e assimétricas de 3 componentes e 6,8 e 10 partes. 

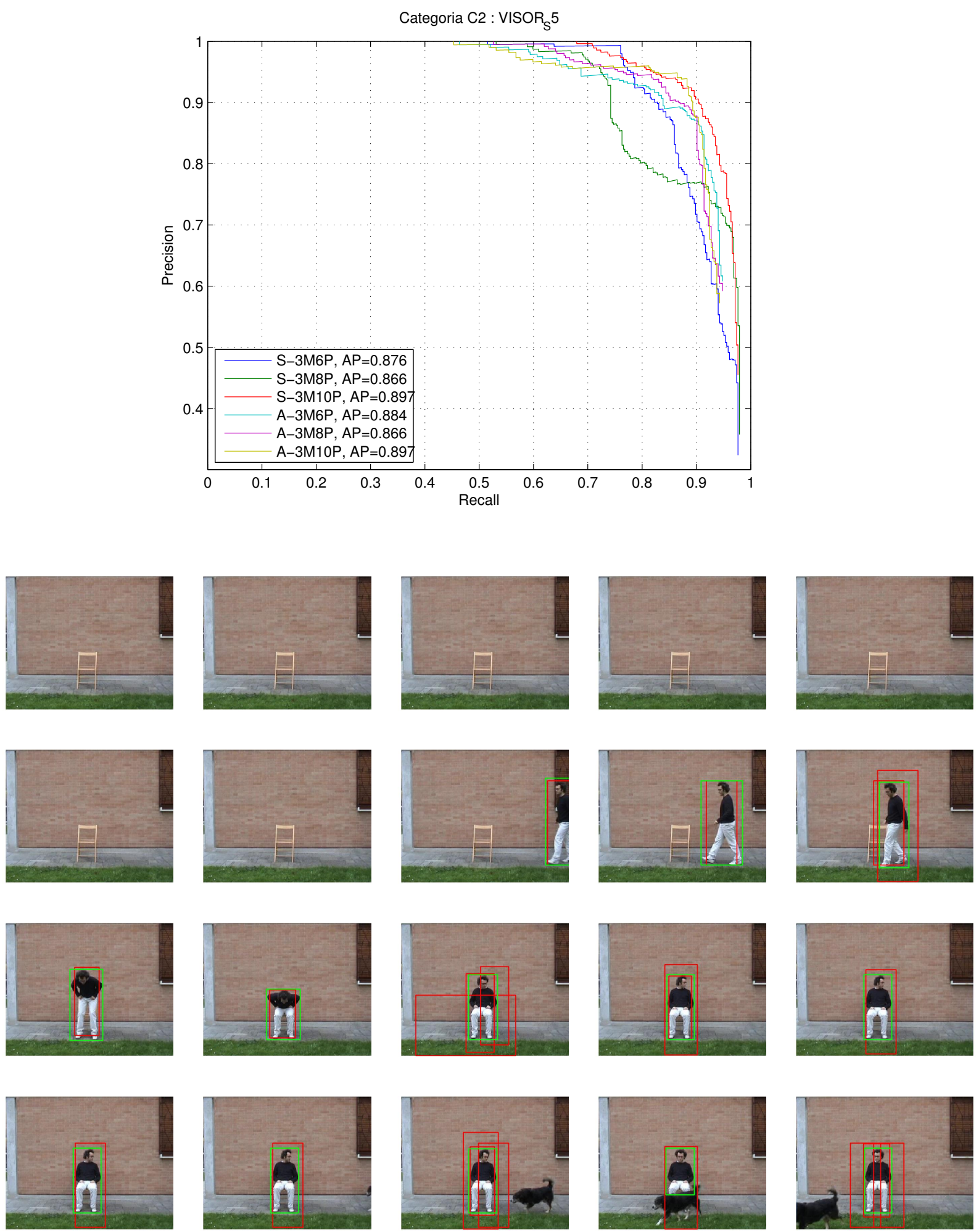

Figura 5.25: Curva de Precision vs. Recall e o AP para o vídeo V26, usando MDPMs simétricas e assimétricas de 3 componentes e 6,8 e 10 partes. 

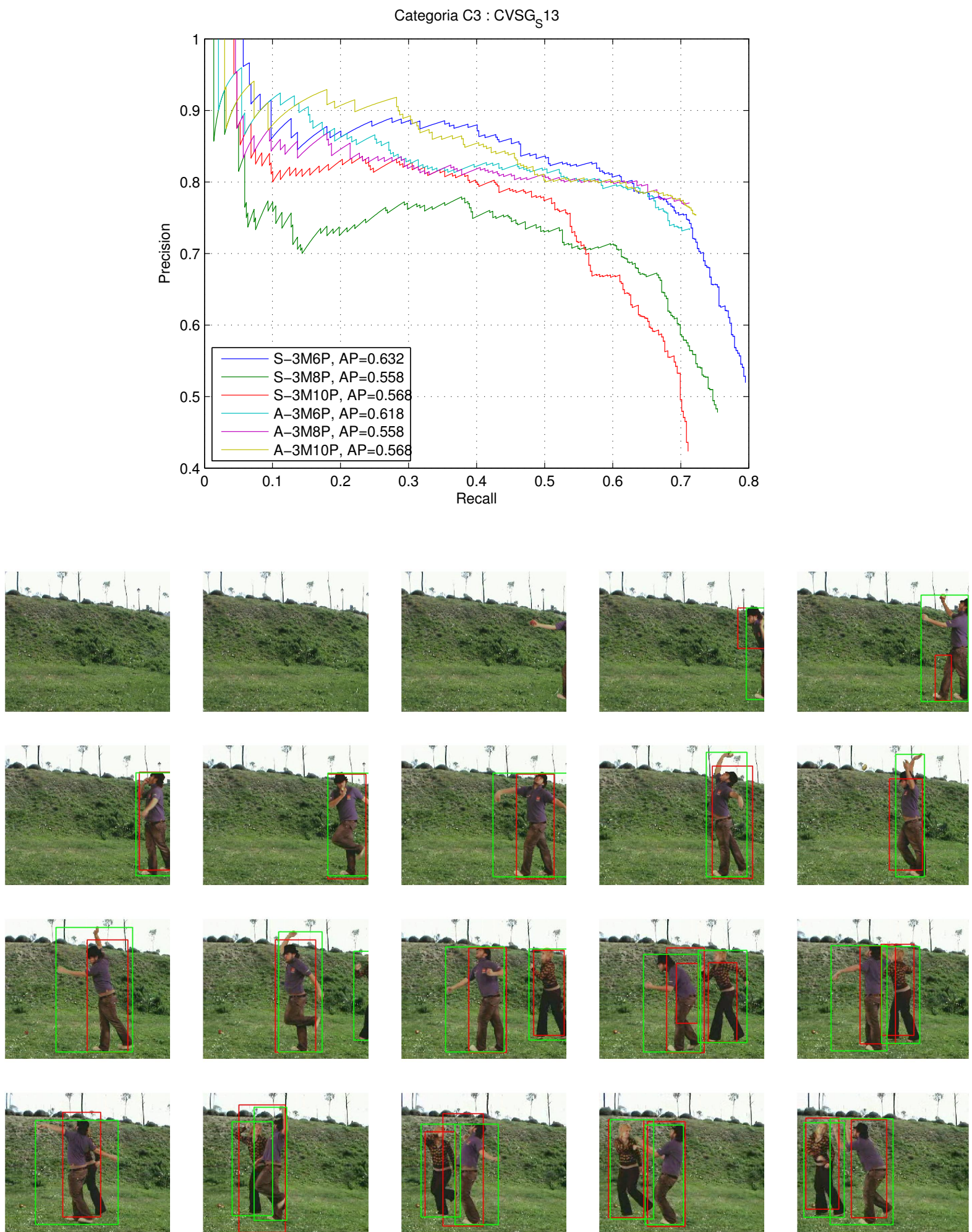

Figura 5.26: Curva de Precision vs. Recall e o AP para o vídeo V31, usando MDPMs simétricas e assimétricas de 3 componentes e 6,8 e 10 partes. 

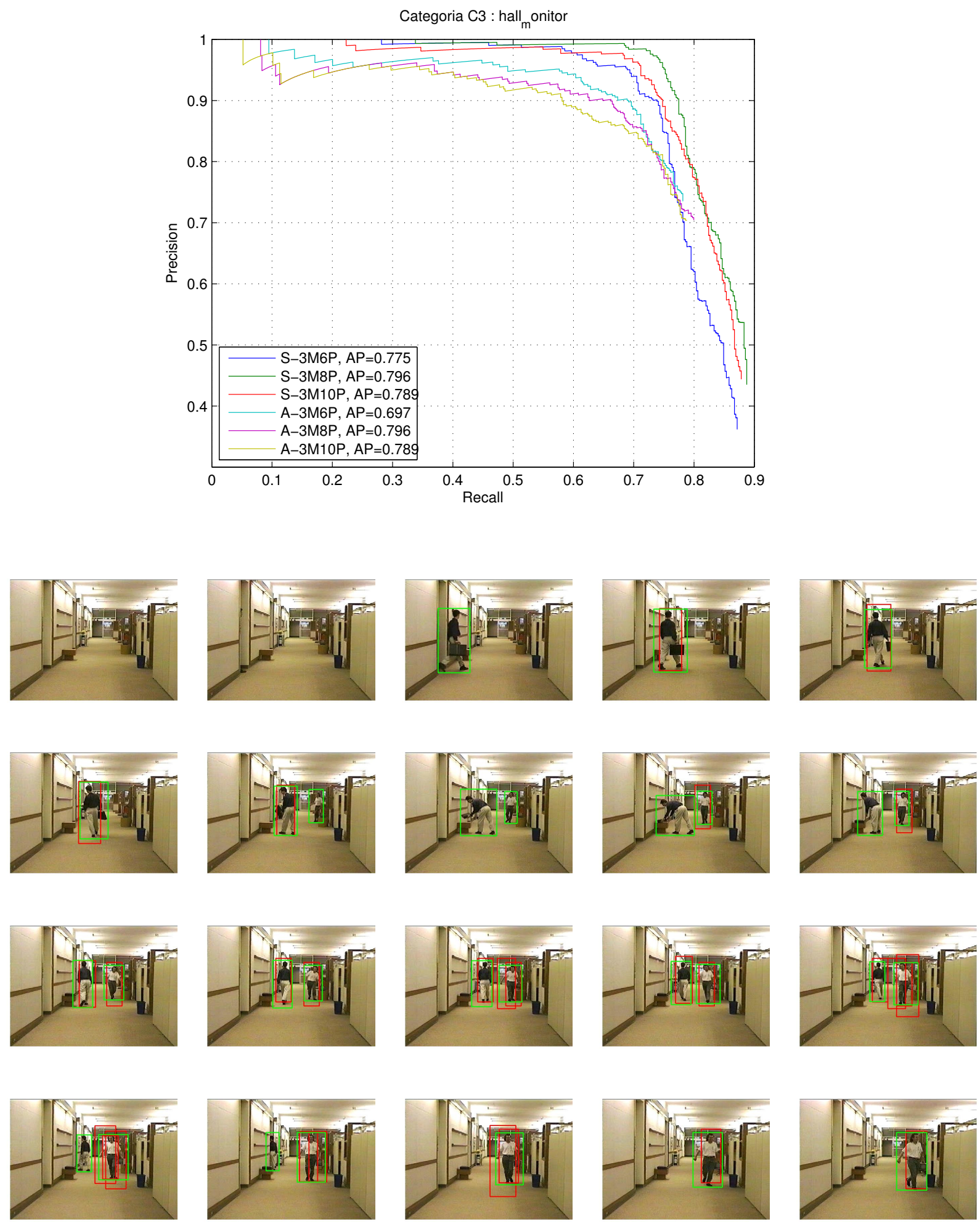

Figura 5.27: Curva de Precision vs. Recall e o AP para o vídeo V32, usando MDPMs simétricas e assimétricas de 3 componentes e 6,8 e 10 partes. 

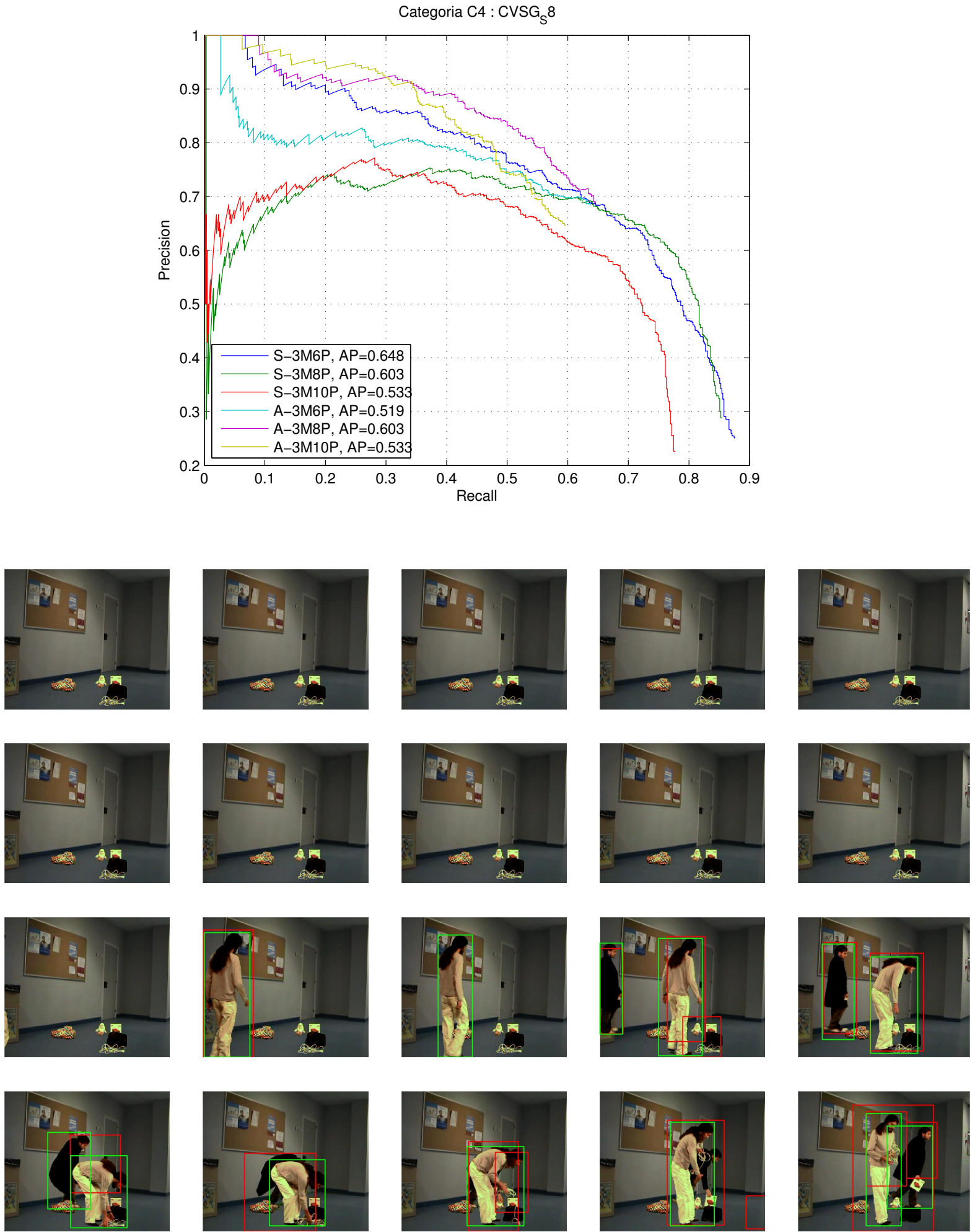

Figura 5.28: Curva de Precision vs. Recall e o AP para o vídeo V43, usando MDPMs simétricas e assimétricas de 3 componentes e 6,8 e 10 partes. 

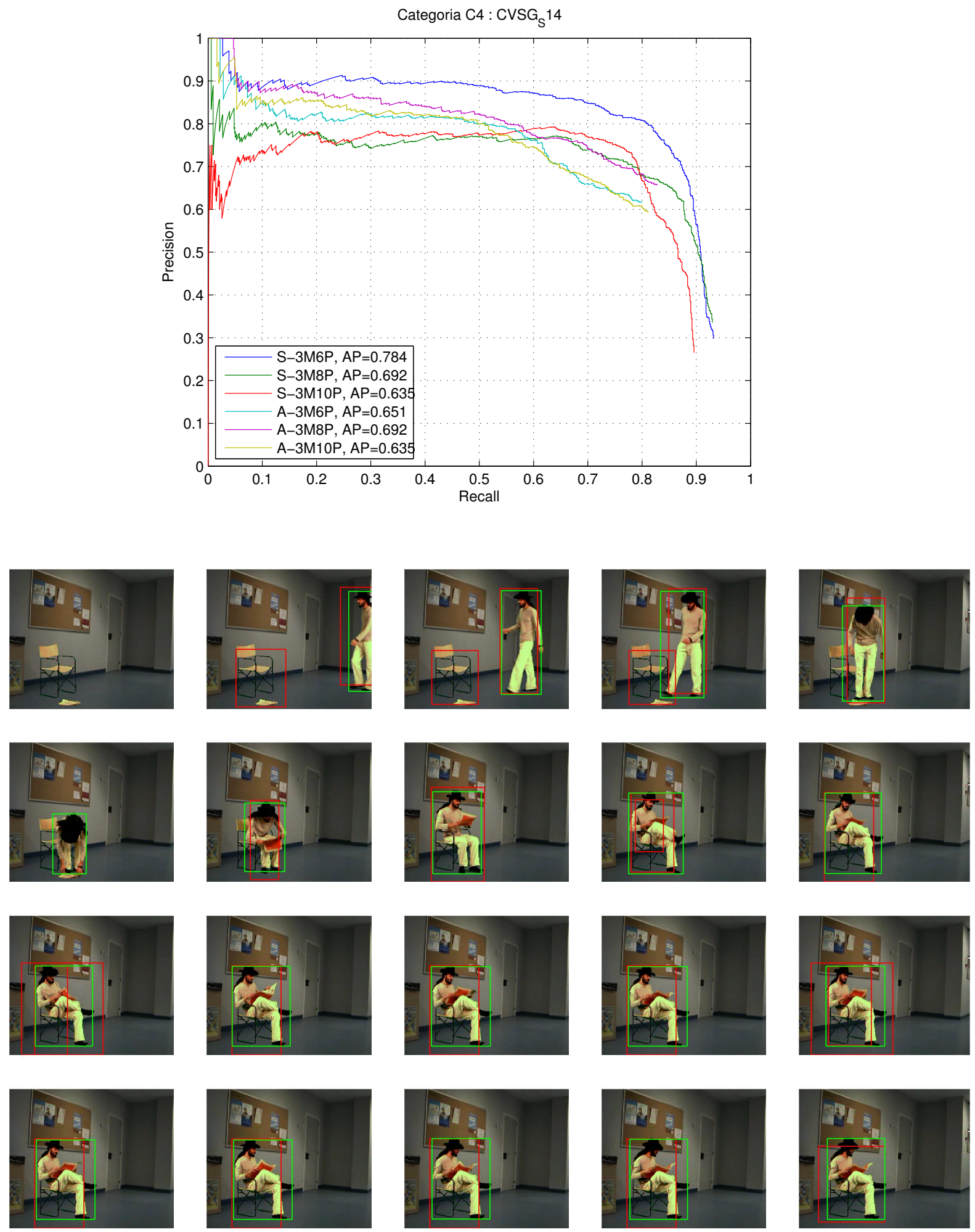

Figura 5.29: Curva de Precision vs. Recall e o AP para o vídeo V44, usando MDPMs simétricas e assimétricas de 3 componentes e 6,8 e 10 partes. 

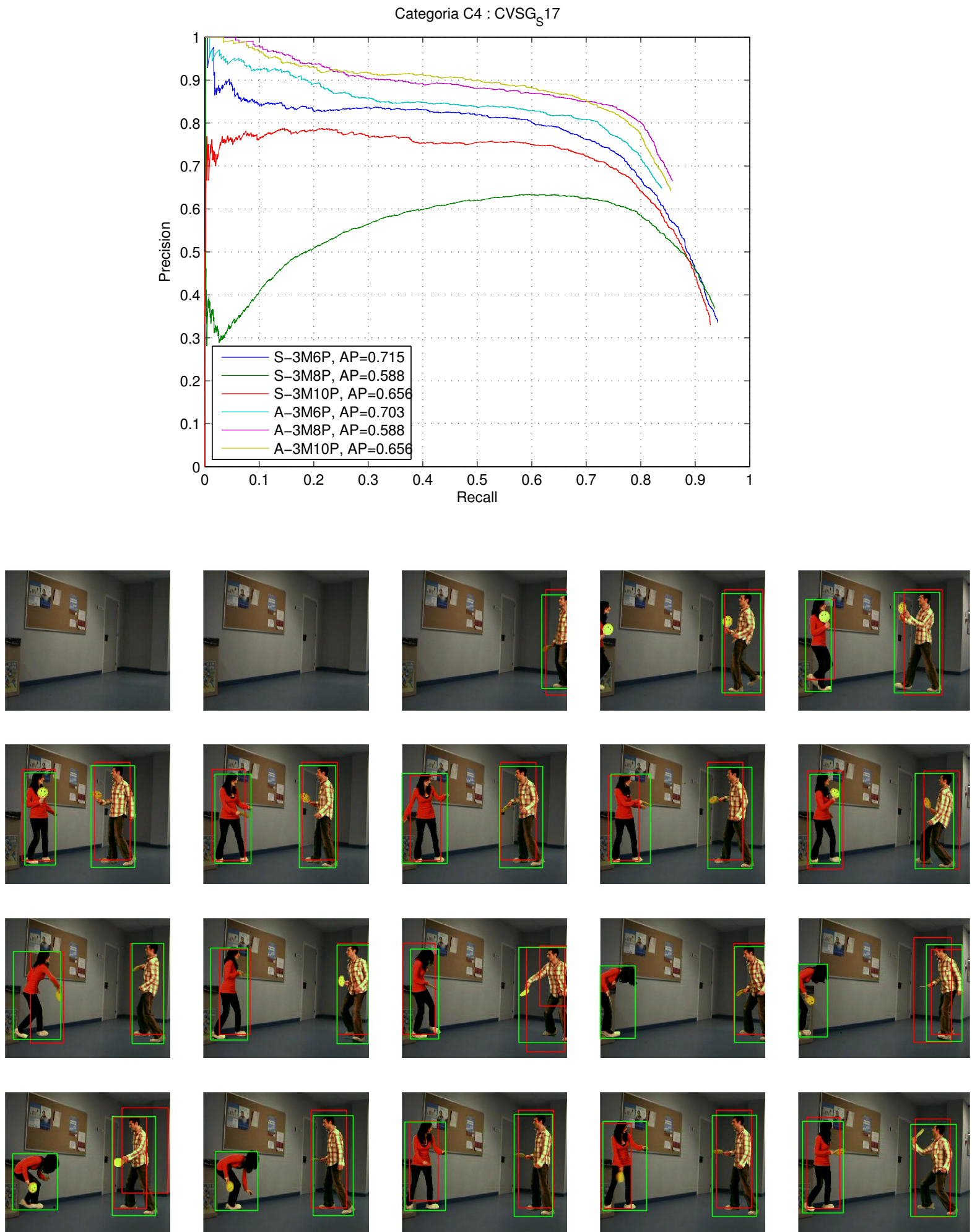

Figura 5.30: Curva de Precision vs. Recall e o AP para o vídeo V45, usando MDPMs simétricas e assimétricas de 3 componentes e 6,8 e 10 partes. 

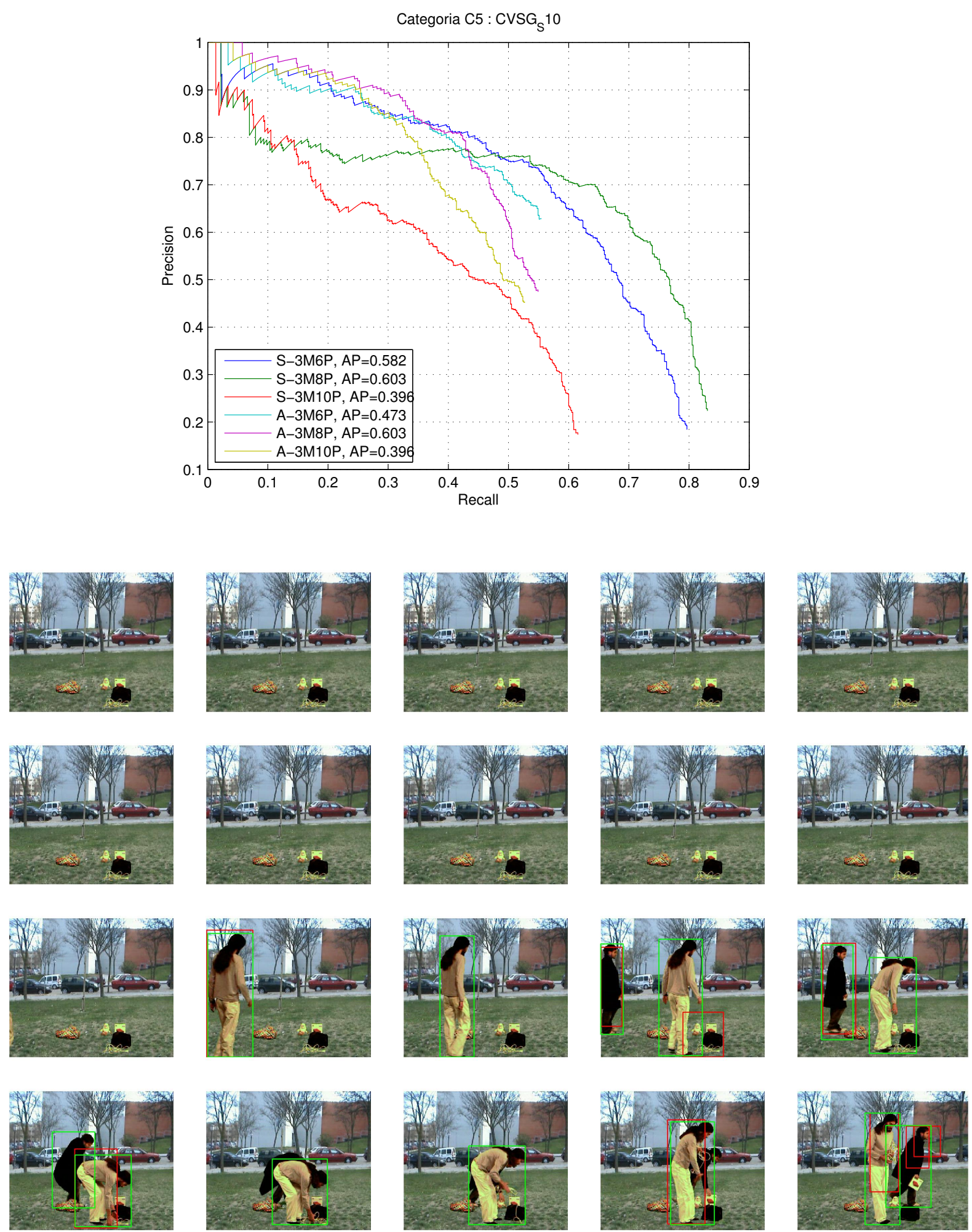

Figura 5.31: Curva de Precision vs. Recall e o AP para o vídeo V52, usando MDPMs simétricas e assimétricas de 3 componentes e 6,8 e 10 partes. 

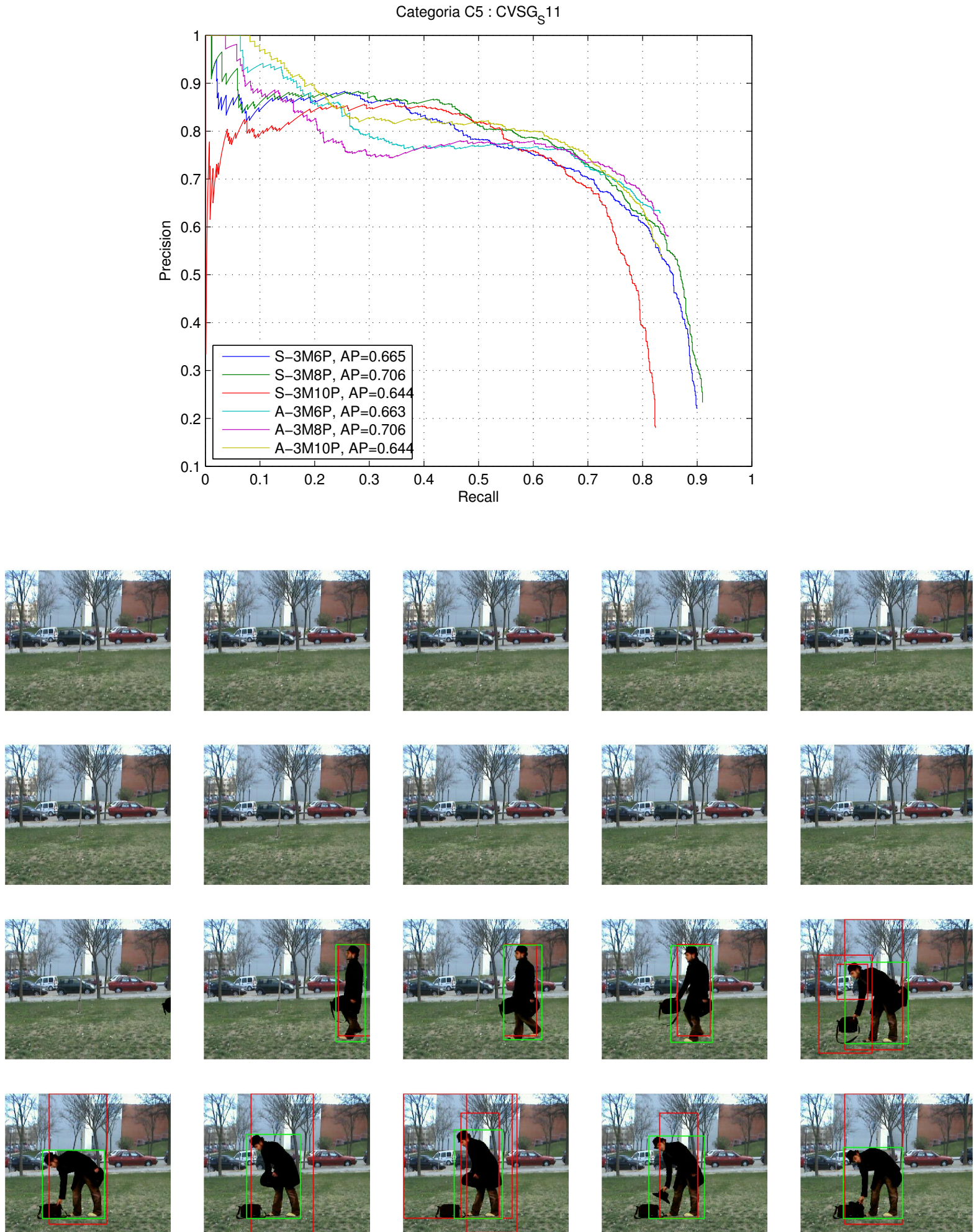

Figura 5.32: Curva de Precision vs. Recall e o AP para o vídeo V53, usando MDPMs simétricas e assimétricas de 3 componentes e 6,8 e 10 partes. 

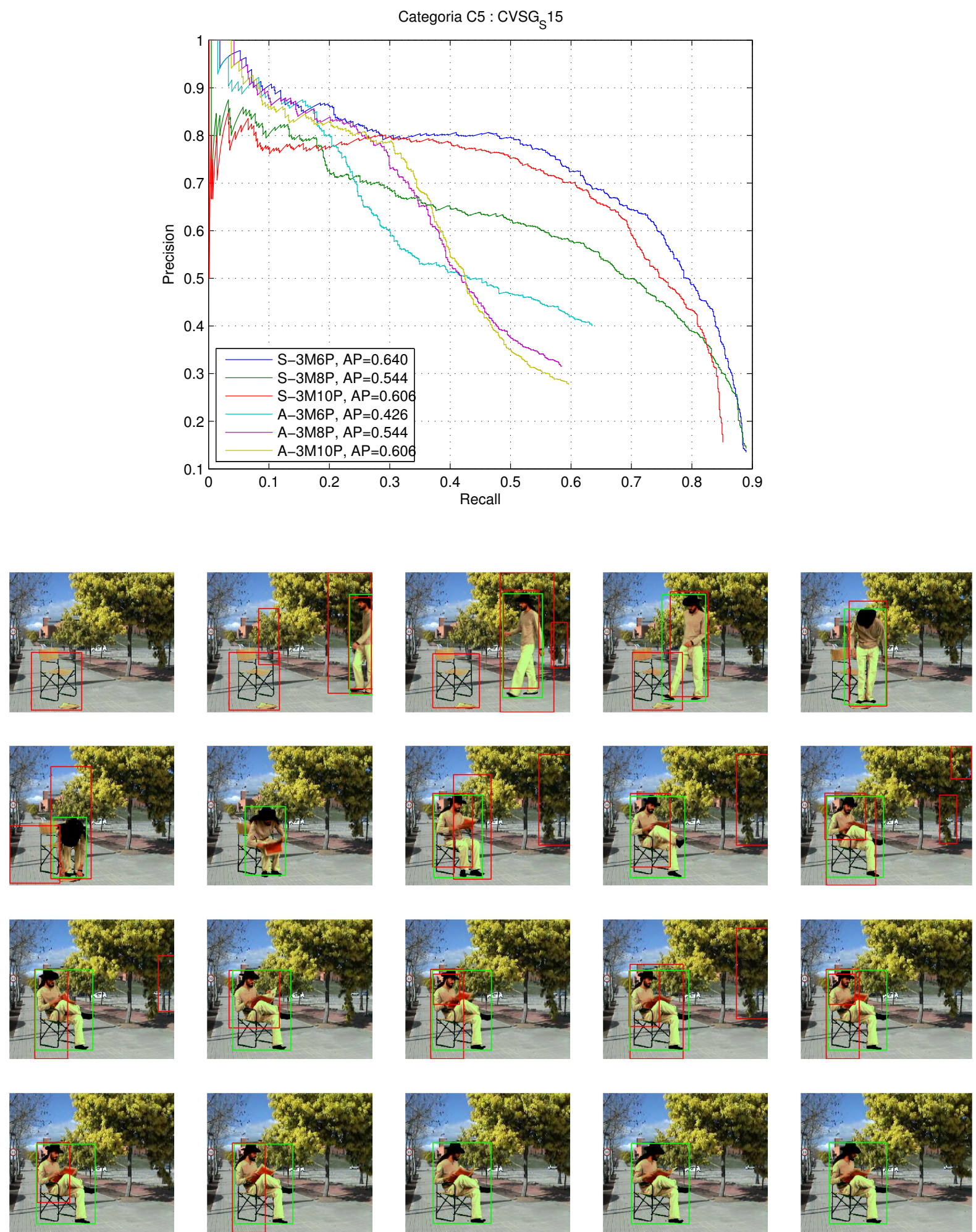

Figura 5.33: Curva de Precision vs. Recall e o AP para o vídeo V54, usando MDPMs simétricas e assimétricas de 3 componentes e 6,8 e 10 partes. 


\subsubsection{Unindo MDPMs simétricas e assimétricas}

Observando alguns resultados obtidos, notamos que algumas vezes o objeto de interesse era detectado usando uma MDPMs simétrica e não com uma MDPM assimétrica e vice-versa. Isto, nos fez pensar em unir ambos e analisar se os resultados melhoram.

Os resultados obtidos para alguns dos vídeos do PDds são apresentados na Tabela 5.13. Como era de esperar o valor de recall aumentou como era já esperado, mas lamentavelmente, a precision, $\mathrm{AP}$ e a pontuação F não. Então, mantem-se a afirmação de que melhores resultados são obtidos considerando MDPMs assimétricas.

\begin{tabular}{|c|c|c|c|c|c|c|c|c|c|c|c|c|}
\hline \multirow[b]{3}{*}{ Vídeo } & \multicolumn{12}{|c|}{ Simétrico + Assimétrico (MDPMs) } \\
\hline & \multicolumn{4}{|c|}{$3 \mathrm{M} 6 \mathrm{P}$} & \multicolumn{4}{|c|}{$3 \mathrm{M} 8 \mathrm{P}$} & \multicolumn{4}{|c|}{$3 \mathrm{M} \mathrm{10P}$} \\
\hline & Pre. & Rev. & AP & $\mathbf{F}$ & Pre. & Rev. & AP & $\mathbf{F}$ & Pre. & Rev. & AP & $\mathbf{F}$ \\
\hline V11 & 0.212 & 0.857 & 0.604 & 0.341 & 0.277 & 0.941 & 0.510 & 0.427 & 0.229 & 0.966 & 0.479 & 0.370 \\
\hline V12 & 0.223 & 0.985 & 0.905 & 0.364 & 0.203 & 0.985 & 0.702 & 0.337 & 0.215 & 0.993 & 0.737 & 0.353 \\
\hline V13 & 0.231 & 0.969 & 0.815 & 0.373 & 0.276 & 0.959 & 0.824 & 0.429 & 0.237 & 0.959 & 0.894 & 0.380 \\
\hline V14 & 0.220 & 0.984 & 0.831 & 0.360 & 0.238 & 0.991 & 0.815 & 0.384 & 0.214 & 0.984 & 0.835 & 0.352 \\
\hline V21 & 0.201 & 0.997 & 0.591 & 0.334 & 0.226 & 1.000 & 0.495 & 0.369 & 0.215 & 1.000 & 0.627 & 0.353 \\
\hline V22 & 0.202 & 0.980 & 0.744 & 0.336 & 0.245 & 0.980 & 0.597 & 0.392 & 0.234 & 1.000 & 0.762 & 0.379 \\
\hline $\mathrm{V} 23$ & 0.251 & 0.929 & 0.686 & 0.395 & 0.267 & 0.911 & 0.645 & 0.413 & 0.247 & 0.900 & 0.671 & 0.388 \\
\hline V31 & 0.336 & 0.841 & 0.588 & 0.480 & 0.338 & 0.847 & 0.510 & 0.484 & 0.315 & 0.831 & 0.578 & 0.457 \\
\hline V32 & 0.253 & 0.878 & 0.654 & 0.393 & 0.282 & 0.894 & 0.613 & 0.428 & 0.289 & 0.896 & 0.646 & 0.437 \\
\hline V41 & 0.205 & 0.945 & 0.743 & 0.337 & 0.212 & 0.930 & 0.645 & 0.345 & 0.186 & 0.927 & 0.629 & 0.309 \\
\hline V44 & 0.217 & 0.960 & 0.717 & 0.354 & 0.237 & 0.955 & 0.623 & 0.380 & 0.197 & 0.933 & 0.629 & 0.325 \\
\hline V45 & 0.233 & 0.956 & 0.643 & 0.374 & 0.249 & 0.956 & 0.496 & 0.395 & 0.231 & 0.956 & 0.620 & 0.372 \\
\hline
\end{tabular}

Tabela 5.13: Precision, recall, AP e a pontuação $F$ obtidos para alguns dos vídeos do PDds, mudando os parâmetros das MDPMs obtidos ao unir as MDPMs simétricas e assimétricas. 


\section{Conclusões}

Neste capítulo resumimos as principais contribuições alcançadas pela dissertação, analisamos os resultados gerais obtidos, extraindo algumas conclusões e propondo algumas futuras pesquisas para estender o trabalho.

\subsection{Resumo das contribuições}

Como resultado da dissertação temos as seguintes principais contribuições:

- Levantamento bibliográfico do estado do arte referente a modelos de objetos e referente à detecção de objetos em imagens e vídeos,

- experimentos comparativos na criação e teste de diferentes MDPMs em imagens. As diferentes MDPMs foram obtidas variando o número de componentes da mistura e o número de partes do modelo para cada uma das classes definidas no trabalho,

- a aplicação do método das MDPMs para a deteç̧ão de objetos em vídeos,

- o definição de um conjunto de experimentos que explora o melhor número de componentes e partes do modelo, em MDPMs simétricas e assimétricas sobre diferentes tipos de vídeo obtidos por uma câmera estacionária ou dinâmica,

- o estudo comparativo do desempenho das MDPMs simétricas e assimétricas, com outros modelos em diferentes tipos de vídeo,

- anotações de carros para sequências do vídeo AVSS_PV_EV AL.avi de CAST e CPNI (2007), as quais encontram-se disponíveis no seguinte site: https://www.dropbox.com/sh/5nozkn3kbk1dtc9/ RjVqAt_0sH, junto com algumas funções para usá-las com MATLAB.

Além das atividades mencionadas, foi publicado o artigo Leon e Hirata (2012), no SIBGRAPI 2012, e apresentado um pôster na escola de verão: "INRIA Visual Recognition and Machine Learning Summer School - VRML" realizada em Grenoble, França, entre os dias 9 e 13 de julho do 2012. Maiores detalhes sobre a escola podem ser encontrados no seguinte link: http://www.di.ens.fr/ willow/events/cvml2012/.

Com o artigo do SIBGRAPI 2012, recebemos um convite para escrever uma extensão do mesmo, no Journal of Pattern Recognition Letter.

\subsection{Conclusões}

O processo de deteç̧ão de objetos é uma área atualmente muito estudada por causa dos diversos âmbitos de aplicação. Adicionalmente, é um problema ainda difícil de resolver e somente impondo 
algumas restrições, o problema pode ser relaxado um pouco. Em relação ao problema de deteç̧ão de objetos em imagens, ou vídeos, um grande número de referencias pode ser encontrado. O estudo realizado focou-se na criação de modelos para representar uma classe de objetos usando um conjunto de imagens rotuladas com a localização do objeto na imagem (bounding boxes), para posteriormente detectar objetos da mesma classe em sequências de vídeo.

Um dos objetivos foi criar um modelo do objeto que melhor o represente o mundo real, para que ele possa ser aplicado em diferentes sequências de vídeo. O problema inicial é escolher o conjunto de imagens para isso. De acordo com o trabalho de Torralba e Efros (2011), optamos por usar o conjunto de imagens PASCAL de Everingham et al. (2010). Em seguida, surgiu o problema de qual técnica usaríamos para a criação de modelos. De acordo com os resultados obtidos no PASCAL VOC 2008, 2010 e no estado da arte, escolheu-se as misturas de modelos de partes deformáveis (MDPMs) de Felzenszwalb et al. (2010b). Além disso, observamos que, na literatura, a criação do modelo é limitada para determinados pontos de visada do objeto ou, então, opta-se por criar vários detectores, um para cada linha de visada (Cho et al., 2011; López-Sastre et al., 2011). Isto é outro ponto em que nos diferençamos da literatura, dado que não limitamos o modelo para uma determinada linha de visada.

Exploramos os parâmetros, tanto das MDPMs simétricas como das assimétricas, fazendo experimentos comparativos variando o número de componentes da mistura e o número de partes do modelo para cada uma das classes definidas no trabalho. Exploramos diferentes sequências de vídeo existentes na literatura. Selecionamos algumas destas, que foram obtidas tanto por câmera estática, como dinâmica, para detectar as classes pessoa ou carro em execuções independentes. As anotações de carros para as sequências do vídeo $A V S S_{-} P V_{-} E V A L$.avi de CAST e CPNI (2007), encontramse disponíveis em Castaneda e Hirata (2013). Adicionalmente o nosso código encontra-se disponível em: http://www.vision.ime.usp.br/ leissicl/code/.

Finalmente, os resultados, em geral, mostraram um bom desempenho nas diferentes sequências de vídeo. Por exemplo, na detecção de carros no vídeo Leuven Leibe et al. (2007), os resultados são equivalentes aos obtidos em Cornelis et al. (2008) e Leibe et al. (2006) (sem usar informação do plano 3D). Com relação às MDPMs, em geral, os melhores resultados foram obtidos pelas MDPMs assimétricas, e regularmente por MDPMs de três componentes e oito e seis partes. Adicionalmente, alguns experimentos realizados indicaram inicialmente que a combinação das MDPMs simétricas e assimétricas poderia dar melhores resultados mas, segundo nossos experimentos, só há melhora na revocação. Por outro lado, dado que os autores de um trabalho similar (Geiger et al., 2011) disponibilizaram seus modelos, conseguimos testar os nossos modelos com os deles. Os modelos deles foram gerados considerando imagens obtidas por uma câmera dinâmica. Desta forma, conseguimos mostrar que ao testar esses modelos em sequências de vídeo obtidos por uma câmera estacionaria, foram obtidos resultados ruins. Pelo contrario, com os nossos modelos criados bons resultados foram obtidos tanto em sequências de vídeo obtidos por câmera estática como dinâmica.

\subsection{Possíveis extensões}

Com relação aos resultados obtidos, algumas extensões poderiam ser feitas:

- Para cada filtro parte, poderia ser escolhida a orientação predominante e logo rotacionar cada parte. Treinar de tal forma que, por exemplo, exista um intervalo de orientações possíveis, a serem consideradas no processo de teste.

- Outra forma de avaliar o número de componentes de uma mistura seria avaliando em que componente tem-se maior detecção.

- Testar os resultados obtidos com outros algoritmos do estado do arte, sobre as mesmas sequências de vídeo. Adicionalmente, considerar outras classes de objetos.

- Para o caso de sequências de vídeo obtidas com uma câmera dinâmica, poderia-se considerar 
a informação do plano 3D. A informação poderia ser considerada para definir a região de detecção, ou como um passo de pós-processamento, para eliminar falsos positivos.

- Considerar alguma técnica que permita "rastrear" os bounding boxes dos objetos através dos quadros do vídeo. Isto poderia ajudar a detectar aqueles objetos que não foram detectados em quadros consecutivos e, também, a uniformizar o tamanho dos bounding boxes.

- Estender para classificação ou busca de vídeos. O primeiro caso refere-se a encontrar no vídeo os quadros em que o objeto desejado aparece. No caso da busca de vídeos, poderia ser considerada uma classificação com um passo adicional, que seria uma heurística para devolver ao usuário os vídeos com maior frequência da presença de um determinado objeto. 
CONCLUSÕES 


\section{Máquinas de vetores de suporte}

"Um programa de computador "aprende" da experiência $E$ com respeito a uma classe de tarefas $T$ e uma medida de performance $P$, se sua performance das tarefas $T$, medida por $P$, melhora com a experiência $E^{\prime \prime}$.

Tom Mitchel.(Mitchell, 1997)

As máquinas de vetores de suporte (SVM) (Vapnik, 1995) é um método usado para resolver problemas de classificação de padrões e problemas de regressão. Neste capítulo apresentamos o SVM linear para classes não separáveis.

\section{A.1 SVM linear}

Dado o conjunto de dados de treinamento $T=\left\{\left(\mathbf{x}_{1}, y_{1}\right), \ldots,\left(\mathbf{x}_{m}, y_{m}\right)\right\}, \mathbf{x}_{i} \in \mathbb{R}^{n}$ e $y \in\{-1,1\}$. Seja o classificador binário definido pela função de decisão:

$$
\begin{aligned}
f_{\text {des }}: \mathbb{R}^{n} & \rightarrow\{-1,1\} \\
\mathbf{x} & \mapsto f_{\text {des }}(x)=\operatorname{sgn}(f(\mathbf{x})),
\end{aligned}
$$

onde

$$
f(\mathbf{x})=\mathbf{w}^{T} \mathbf{x}+b, \quad \mathbf{w} \in \mathbb{R}^{n}, b \in \mathbb{R} .
$$

No caso que os dados não sejam linearmente separáveis, isto é, dados que não podem ser separados totalmente por um hiperplano, a formulação do SVM é a seguinte:

$$
\min _{\mathbf{w}, b} \frac{1}{2}\|\mathbf{w}\|^{2}+C \sum_{i=1}^{m} \max \left(0,1-y_{i} f\left(\mathbf{x}_{i}\right)\right)
$$

restrito a

$$
y_{i}\left(\mathbf{w}^{T} x_{i}+b\right) \geq 1, \quad i=1,2, \ldots, m,
$$

onde $\max \left(0,1-y_{i} f(\mathbf{x})\right)$ representa uma função de custo ou perda("hinge loss"), e $C$ é um parâmetro que controla o tamanho do margem tal que o erro seja pequeno.

Assim, (i) se $y f(\mathbf{x}) \geq 1$ (classificação totalmente correta) então $\max (0,1-y f(\mathbf{x}))=0$. (ii) Se $0 \leq y f(\mathbf{x})<1$ ou (iii) se houver uma classificação totalmente errônea, então a função de custo cresce com $1-y f(\mathbf{x})$. Quando, o custo não é diferenciável, introduz-se variáveis de folga (slack) $\xi_{i}$. Logo, o objetivo do SVM linear é reescrito como:

$$
\min \frac{1}{2}\|\mathbf{w}\|^{2}+C \sum_{i=1}^{m} \xi_{i}
$$


sobre a restrição $\xi_{i} \geq \max \left(0,1-y_{i} f\left(\mathbf{x}_{i}\right)\right)$, o qual é equivalente a duas restrições:

$$
\begin{gathered}
\xi_{i} \geq 0 \quad \forall i=1,2, \ldots, m, \mathrm{e} \\
\xi_{i} \geq 1-y_{i} f\left(\mathbf{x}_{i}\right) \quad \forall i=1,2, \ldots, m .
\end{gathered}
$$

Para resolver o problema definido na Equação (A.5), usa-se o método dos multiplicadores de Lagrange $\alpha$ e $\beta$ sobre as condições de Karush-Kuhn-Tucker (KKT) (veja em Theodoridis e Koutroumbas (2003)). A função de Lagrange é definida como:

$$
\mathfrak{L}(\mathbf{w}, b, \xi, \alpha, \beta)=\frac{1}{2} \mathbf{w}^{T} \mathbf{w}-\sum_{i=1}^{m} \alpha_{i}\left[\xi+y_{i} f\left(\mathbf{x}_{i}\right)-1\right]+C \sum_{i=1}^{m} \xi_{i}-\beta_{i} \sum_{i=1}^{m} \xi_{i}
$$

sobre as condições:

$$
\begin{gathered}
\frac{\delta \mathfrak{L}}{\delta \mathbf{w}}(\mathbf{w}, b, \xi, \alpha, \beta)=0 \quad \text { ou } \quad \mathbf{w}=\sum_{i=1}^{m} \alpha_{i} y_{i} \mathbf{x}_{i} \\
\frac{\delta \mathfrak{L}}{\delta b}(\mathbf{w}, b, \xi, \alpha, \beta)=0 \quad \text { ou } \quad \sum_{i=1}^{m} \alpha_{i} y_{i}=0 \\
\frac{\delta \mathfrak{L}}{\delta \xi_{i}}(\mathbf{w}, b, \xi, \alpha, \beta)=0 \quad \text { ou } \quad C-\alpha_{i}-\beta_{i}=0, \quad i=1, \ldots, m \\
\alpha_{i}\left[y_{i} f\left(\mathbf{x}_{i}\right)-1+\xi_{i}\right]=0, \quad i=1, \ldots, m \\
\beta_{i} \xi_{i}=0, \quad i=1,2, \ldots, m \\
\alpha_{i} \geq 0, \quad \beta_{i} \geq 0, \quad i=1,2, \ldots, m
\end{gathered}
$$

Substituindo as Equações (A.9), (A.10), (A.11) e (A.14) na formulação dual do problema de otimização (Theodoridis e Koutroumbas, 2003), definida como o $\max \mathfrak{L}(\mathbf{w}, b, \xi, \alpha, \beta)$, é a seguinte:

$$
\begin{gathered}
\max _{\alpha}\left(\sum_{i=1}^{m} \alpha_{i}-\frac{1}{2} \sum_{i, j}^{m} \alpha_{i} \alpha_{j} y_{i} y_{j} \mathbf{x}_{i}^{T} \mathbf{x}_{j}\right), \\
\text { restrito a } \quad 0 \leq \alpha_{i} \leq C, \quad i=1,2, \ldots, m \\
\sum_{i=1}^{m} \alpha_{i} y_{i}=0
\end{gathered}
$$

Finalmente os valores de $\alpha$ obtidos solucionando este problema, permitem calcular o valor $\mathbf{w}$ usando a Equação (A.9), e o valor de $b$ a partir da Equação (A.12). Estos valores são substituídos na função de decisão. 


\section{Referências Bibliográficas}

Amit(2002) Y. Amit. 2D Object Detection and Recognition: Models, Algorithms and Networks. MIT Press. Citado na pág. 8

Amit e Trouve(2007) Y. Amit e A. Trouve. Pop: Patchwork of parts models for object recognition. International Journal of Computer Vision, 75(2):267-282. Citado na pág. 8, 11, 15

Andrews et al.(2003) S. Andrews, I. Tsochantaridis e T. Hofmann. Support vector machines for multiple-instance learning. Em Advances in Neural Information Processing Systems 15, páginas 561-568. MIT Press. Citado na pág. 15

Andriluka et al.(2008) M. Andriluka, S. Roth e B. Schiele. People-tracking-by-detection and people-detection-by-tracking. Em 26th IEEE Conference on Computer Vision and Pattern Recognition, CVPR. Citado na pág. 12, 14, 16

Bay et al.(2006) H. Bay, T. Tuytelaars e L. Van Gool. SURF: Speeded up robust features, volume 3951 LNCS of Lecture Notes in Computer Science (including subseries Lecture Notes in Artificial Intelligence and Lecture Notes in Bioinformatics). Citado na pág. 9

Beis e Lowe(1997) J. S. Beis e D. G. Lowe. Shape indexing using approximate nearest-neighbour search in high-dimensional spaces. Em Proceedings of the 1997 Conference on Computer Vision and Pattern Recognition (CVPR '97), CVPR '97, páginas 1000-, Washington, DC, USA. IEEE Computer Society. ISBN 0-8186-7822-4. Citado na pág. 13

Bentley(1975) J. L. Bentley. Multidimensional binary search trees used for associative searching. Commun. ACM, 18:509-517. Citado na pág. 13

Bosch et al.(2007) A. Bosch, A. Zisserman e X. Munoz. Representing shape with a spatial pyramid kernel. Em Proceedings of the 6th ACM International Conference on Image and Video Retrieval, CIVR 200\%, páginas 401-408. Citado na pág. 10, 12

Bursuc et al.(2011) A. Bursuc, T. Zaharia e F. Prêteux. Detection of multiple instances of video objects. Em Proceedings - 7th International Conference on Signal Image Technology and Internet-Based Systems, SITIS 2011, páginas 446-453. Citado na pág. 2

CAST e CPNI(2007) CAST e CPNI. i-lids dataset for avss 2007. ftp://motinas.elec.qmul.ac. uk/pub/iLids, 2007. Citado na pág. 34, 83, 84

Castaneda e Hirata(2013) L. Castaneda e R. Hirata. Site of results. http://www.vision.ime. usp.br/ leissicl/annotations.html, 2013. Citado na pág. 84 
Chan e Vasconcelos(2005) A. B. Chan e N. Vasconcelos. Probabilistic kernels for the classification of auto-regressive visual processes. Em Proceedings - 2005 IEEE Computer Society Conference on Computer Vision and Pattern Recognition, CVPR 2005, volume I, páginas 846851. Citado na pág. xvii, 33,34

Chang e Lin(2011) C. . Chang e C. . Lin. LIBSVM: A library for support vector machines. ACM Transactions on Intelligent Systems and Technology, 2:27:1-27:27. Software available at http://www.csie.ntu.edu.tw/ cjlin/libsvm. Citado na pág. 13

Chen et al.(2010) Y. Chen, L. Zhu e A. Yuille. Active mask hierarchies for object detection. Em Proceedings of the 11th European conference on Computer vision: Part V, ECCV'10, páginas 43-56. Citado na pág. 43

Cheung e Kamath(2004) S.-Ch. S. Cheung e Ch. Kamath. Robust techniques for background subtraction in urban traffic video. volume 5308, páginas 881-892. SPIE. URL http://dx.doi.org/ 10.1117/12.526886. Citado na pág. 3, 32

Cho et al.(2011) H. Cho, P. Rybski e W. Zhang. Vision-based 3d bicycle tracking using deformable part model and interacting multiple model filter. Em 2011 IEEE Conference on Robotics and Automation (ICRA 2011). Citado na pág. 84

Collins et al.(1999) R. Collins, A. Lipton e T. Kanade. A System for Video Surveillance and Monitoring. Em American Nuclear Society 8th Internal Topical Meeting on Robotics and Remote Systems. Citado na pág. 3

Constantine e Tomaso(2000) P. Constantine e P. Tomaso. A trainable system for object detection. International Journal of Computer Vision, 38(1):15-33. URL http://www.springerlink. com/index/WW5UT522540L672R.pdf. Citado na pág. 3, 9, 10, 14, 15

Cootes e Taylor(2001) T. F. Cootes e C. J. Taylor. Constrained active appearance models. Em Proceedings of the IEEE International Conference on Computer Vision, volume 1, páginas 748-754. Citado na pág. 11

Cornelis et al.(2008) N. Cornelis, B. Leibe, K. Cornelis e L. Van Gool. 3d urban scene modeling integrating recognition and reconstruction. International Journal of Computer Vision, 78(2-3): 121-141. Citado na pág. 3, 62, 84

Cortes e Vapnik(1995) C. Cortes e V. Vapnik. Support-vector networks. Machine Learning, 20 (3):273-297. Citado na pág. 13

Coughlan et al.(2000) J. Coughlan, A. Yuille, C. English e D. Snow. Efficient deformable template detection and localization without user initialization. Computer Vision and Image Understanding, 78(3):303-319. Citado na pág. 8, 11, 15

Crandall et al.(2005) D. Crandall, P. Felzenszwalb e D. Huttenlocher. Spatial priors for part-based recognition using statistical models. Em Proceedings of the IEEE Computer Society Conference on Computer Vision and Pattern Recognition, volume 1, páginas 10-17. Citado na pág. 8, 11, 15

Crandall e Huttenlocher(2006) D. J. Crandall e D. P. Huttenlocher. Weakly supervised learning of part-based spatial models for visual object recognition, volume 3951 LNCS of Lecture Notes in Computer Science (including subseries Lecture Notes in Artificial Intelligence and Lecture Notes in Bioinformatics). Citado na pág. 11

Dalal(2006) N. Dalal. Finding people in images and videos. Tese de Doutorado, Institut National Polytechnique de Grenoble. URL http://www.navneetdalal.com/. Citado na pág. 1, 3, 8, 10, 15, 16 
Dalal e Triggs(2005) N. Dalal e B. Triggs. Histograms of oriented gradients for human detection. Em Proceedings - 2005 IEEE Computer Society Conference on Computer Vision and Pattern Recognition, CVPR, páginas I: 886-893. URL http://dx.doi.org/10.1109/CVPR.2005.177. Citado na pág. $3,10,14,15,32$

Dalal et al.(2006) N. Dalal, B. Triggs e C. Schmid. Human detection using oriented histograms of flow and appearance, volume 3952 LNCS of Lecture Notes in Computer Science (including subseries Lecture Notes in Artificial Intelligence and Lecture Notes in Bioinformatics). Citado na pág. 3

Dickinson(2009) S Dickinson. The Evolution of Object Categorization and the Challenge of Image Abstraction, páginas 1-37. Cambridge University Press. Citado na pág. 8

Dollár et al.(2012) P. Dollár, C. Wojek, B. Schiele e P. Perona. Pedestrian detection: An evaluation of the state of the art. IEEE Transactions on Pattern Analysis and Machine Intelligence, 34(4): 743-761. Citado na pág. 1, 14

Everingham et al.(a) M. Everingham, L. Van Gool, C. K. I. Williams, J. Winn e A. Zisserman. The PASCAL Visual Object Classes Challenge 2007. http://pascallin.ecs.soton.ac.uk/challenges/VOC/voc2007/, a. Citado na pág. xvii, 31

Everingham et al.(b) M. Everingham, L. Van Gool, C. K. I. Williams, J. Winn e A. Zisserman. The PASCAL Visual Object Classes Challenge 2008. http://pascallin.ecs.soton.ac.uk/challenges/VOC/voc2008/, b. Citado na pág. 14

Everingham et al.(2010) M. Everingham, L. Van Gool, C. K. I. Williams, J. Winn e A. Zisserman. The pascal visual object classes (voc) challenge. International Journal of Computer Vision, 88 (2):303-338. Citado na pág. xiii, xiv, 1, 2, 4, 8, 30, 84

Fei-Fei et al.(2003) L. Fei-Fei, R. Fergus e P. Perona. A bayesian approach to unsupervised one-shot learning of object categories. Em Proceedings of the IEEE International Conference on Computer Vision, volume 2, páginas 1134-1141. Citado na pág. 8

Felzenszwalb et al.(2008) P. Felzenszwalb, D. McAllester e D. Ramanan. A discriminatively trained, multiscale, deformable part model. Em IEEE Conference on Computer Vision and Pattern Recognition (CVPR). Citado na pág. xiii, 3, 10, 11, 12, 14, 15, 22

Felzenszwalb e Huttenlocher(2005) P. F. Felzenszwalb e D. P. Huttenlocher. Pictorial structures for object recognition. International Journal of Computer Vision, 61(1):55-79. Citado na pág. xiii, $8,11,12,14,15$

Felzenszwalb e Schwartz(2007) P. F. Felzenszwalb e J. D. Schwartz. Hierarchical matching of deformable shapes. Em Proceedings of the IEEE Computer Society Conference on Computer Vision and Pattern Recognition. Citado na pág. 8, 15

Felzenszwalb et al.() P. F. Felzenszwalb, R. B. Girshick e D. McAllester. Discriminatively trained deformable part models, release 4 . http://people.cs.uchicago.edu/ pff/latent-release4/. Citado na pág. $10,11,15,17,41$

Felzenszwalb et al.(2010a) P. F. Felzenszwalb, R. B. Girshick e D. McAllester. Cascade object detection with deformable part models. Em Proceedings of the IEEE Computer Society Conference on Computer Vision and Pattern Recognition, páginas 2241-2248. Citado na pág. 14

Felzenszwalb et al.(2010b) Pedro F. Felzenszwalb, Ross B. Girshick, David McAllester e Deva Ramanan. Object detection with discriminatively trained part-based models. IEEE Trans. Pattern Anal. Mach. Intell., 32(9):1627-1645. ISSN 0162-8828. doi: 10.1109/TPAMI.2009.167. URL http://dx.doi.org/10.1109/TPAMI.2009.167. Citado na pág. v, vii, xiii, 1, 3, 9, 10, 11, 13, 14, 15, $16,17,20,21,22,41,84$ 
Fergus et al.(2003) R. Fergus, P. Perona e A. Zisserman. Object class recognition by unsupervised scale-invariant learning. Em Proceedings of the IEEE Computer Society Conference on Computer Vision and Pattern Recognition, volume 2, páginas II/264-II/271. Citado na pág. 3, 8, 11, 15

Feris et al.(2011) R. Feris, J. Petterson, B. Siddiquie, L. Brown e S. Pankanti. Large-scale vehicle detection in challenging urban surveillance environments. Em Proceedings of the 2011 IEEE Workshop on Applications of Computer Vision (WACV), WACV '11, páginas 527-533. IEEE Computer Society. Citado na pág. 14, 15

Fidler e Leonardis(2007) S. Fidler e A. Leonardis. Towards scalable representations of object categories: Learning a hierarchy of parts. CVPR. Citado na pág. 15

Fischler e Elschlager(1973) M.A. Fischler e R.A. Elschlager. The representation and matching of pictorial structures. IEEE Transactions on Computers, 22:67-92. ISSN 0018-9340. doi: http: //doi.ieeecomputersociety.org/10.1109/T-C.1973.223602. Citado na pág. xiii, 8, 12, 15

Foundation() Free Software Foundation. Gnu general public license. http://www.gnu.org/ copyleft/gpl.html. Último acesso em 30/1/2010. Citado na pág. 39

García-Martín et al.(2012) A. García-Martín, J. M. Martínez e J. Bescós. A corpus for benchmarking of people detection algorithms. Pattern Recognition Letters, 33(2):152-156. Citado na pág. $14,35,65$

García-Martín e Martínez(2012) Á. García-Martín e J. M. Martínez. On collaborative people detection and tracking in complex scenarios. Image and Vision Computing, 30(4-5):345-354. Citado na pág. 1,16

García-Martín et al.(2011) Á. García-Martín, A. Hauptmann e J. M. Martínez. People detection based on appearance and motion models. Em 2011 8th IEEE International Conference on Advanced Video and Signal Based Surveillance, AVSS 2011, páginas 256-260. Citado na pág. 65

Geiger et al.(2011) Andreas Geiger, Christian Wojek e Raquel Urtasun. Joint 3d estimation of objects and scene layout. Em Neural Information Processing Systems (NIPS), Granada, Spain. Citado na pág. xvii, 40, 41, 55, 62, 84

Geiger et al.(to appear) Andreas Geiger, Philip Lenz, Christoph Stiller e Raquel Urtasun. Vision meets robotics: The kitti dataset. International Journal of Robotics Research (IJRR). Citado na pág. 40

Harzallah et al.(2008) H. Harzallah, C. Schmid, F. Jurie e A. Gaidon. Classification aided two stage localization. 2008. Citado na pág. 3, 9, 14, 15

Hussein et al.(2009) M. Hussein, F. Porikli e L. Davis. Object detection via boosted deformable features. Em Proceedings of the 16th IEEE international conference on Image processing, ICIP'09, páginas 1429-1432, Piscataway, NJ, USA. IEEE Press. Citado na pág. 8

Jain et al.(2011) R. Jain, P. K. Sankar e C. V. Jawahar. Interpolation based tracking for fast object detection in videos. Em Proceedings - 2011 3rd National Conference on Computer Vision, Pattern Recognition, Image Processing and Graphics, NCVPRIPG 2011, páginas 102-105. Citado na pág. 15,16

Joachims(1999a) T. Joachims. Making large-scale svm learning practical. Advances in Kernel Methods - Support Vector Learning, páginas 169-184. Citado na pág. 13

Joachims(1999b) T. Joachims. Making large-scale svm learning practical. Em Advances in Kernel Methods - Support Vector Learning. MIT Press. Software available at http://svmlight.joachims. org/. Citado na pág. 13, 25 
Joachims et al.(2005) T. Joachims, T. Galor e R. Elber. Learning to align sequences: A maximummargin approach. Em B. Leimkuhler, editor, New Algorithms for Macromolecular Simulation, LNCS Vol. 49. Springer. Citado na pág. 13

Johnson e Hebert(1999) A. E. Johnson e M. Hebert. Using spin images for efficient object recognition in cluttered 3d scenes. IEEE Transactions on Pattern Analysis and Machine Intelligence, 21(5):433-449. Citado na pág. 9

Kamijo et al.(2001) S. Kamijo, K. Ikeuchi e M. Sakauchi. Vehicle tracking in low-angle and front-view images based on spatio-temporal markov random field model. Em In Proceedings of the 8th World Congress on Intelligent Transportation Systems (ITS. Citado na pág. 3, 32

Ke e Sukthankar(2004) Y. Ke e R. Sukthankar. Pca-sift: A more distinctive representation for local image descriptors. Em Proceedings of the IEEE Computer Society Conference on Computer Vision and Pattern Recognition, volume 2, páginas II506-II513. Citado na pág. 9

Lampert(2009) C. H. Lampert. Detecting objects in large image collections and videos by efficient subimage retrieval. Em Proceedings of the IEEE International Conference on Computer Vision, páginas 987-994. Citado na pág. 2

Lazebnik et al.(2003) S. Lazebnik, C. Schmid e J. Ponce. A sparse texture representation using affine-invariant regions. Em Proceedings of the IEEE Computer Society Conference on Computer Vision and Pattern Recognition, volume 2, páginas II/319-II/324. Citado na pág. 9

Lazebnik et al.(2006) S. Lazebnik, C. Schmid e J. Ponce. Beyond bags of features: Spatial pyramid matching for recognizing natural scene categories. Em Proceedings of the IEEE Computer Society Conference on Computer Vision and Pattern Recognition, volume 2, páginas 2169-2178. Citado na pág. 9

Leibe et al.(2006) B. Leibe, N. Cornelis, K. Cornelis e 1. Van Gool. Integrating recognition and reconstruction for cognitive traffic scene analysis from a moving vehicle. Em DAGM-Symposium, páginas 192-201. Citado na pág. 3, 32, 62, 84

Leibe et al.(2007) B. Leibe, N. Cornelis, K. Cornelis e L. Van Gool. Dynamic 3d scene analysis from a moving vehicle. Em Proceedings of the IEEE Computer Society Conference on Computer Vision and Pattern Recognition. Citado na pág. xiv, 32, 34, 35, 84

Leibe et al.(2008a) B. Leibe, A. Leonardis e B. Schiele. Robust object detection with interleaved categorization and segmentation. International Journal of Computer Vision, 77(1-3):259-289. Citado na pág. $3,8,11,14,15,32$

Leibe et al.(2008b) B. Leibe, K. Schindler, N. Cornelis e L. Van Gool. Coupled object detection and tracking from static cameras and moving vehicles. IEEE Transactions on Pattern Analysis and Machine Intelligence, 30(10):1683-1698. Citado na pág. xiv, 3, 15, 16, 32

Leon e Hirata(2012) Leissi Castaneda Leon e Roberto Jr. Hirata. Vehicle detection using mixture of deformable parts models: Static and dynamic camera. Em SIBGRAPI, páginas 237-244. IEEE Computer Society. ISBN 978-1-4673-2802-9. Citado na pág. 83

Liu et al.(2008) C. Liu, J. Yuen, A. Torralba, J. Sivic e W. T. Freeman. Sift flow: Dense correspondence across different scenes. Em Proceedings of the 10th European Conference on Computer Vision: Part III, ECCV '08, páginas 28-42, Berlin, Heidelberg. Springer-Verlag. Citado na pág. 9

López-Sastre et al.(2011) R. J. López-Sastre, T. Tuytelaars e S. Savarese. Deformable part models revisited: A performance evaluation for object category pose estimation. Em ICCV Workshops, páginas 1052-1059. Citado na pág. 14, 15, 84 
Lowe(2004) D. G. Lowe. Distinctive image features from scale-invariant keypoints. International Journal of Computer Vision, 60(2):91-110. Citado na pág. 9, 13, 14

Lowe(1999) D. G. Lowe. Object recognition from local scale-invariant features. Em Proceedings of the IEEE International Conference on Computer Vision, volume 2, páginas 1150-1157. Citado na pág. 9,14

Lv et al.(2012) Yang Lv, Benjamin Yao, Yongtian Wang e Song-Chun Zhu. Reconfigurable templates for robust vehicle detection and classification. Em Proceedings of the 2012 IEEE Workshop on the Applications of Computer Vision, WACV '12, páginas 321-328, Washington, DC, USA. IEEE Computer Society. ISBN 978-1-4673-0233-3. doi: 10.1109/WACV.2012.6163016. URL http://dx.doi.org/10.1109/WACV.2012.6163016. Citado na pág. 15

Magee(2004) D. R. Magee. Tracking multiple vehicles using foreground, background and motion models. Image Vision Comput., 22(2):143-155. Citado na pág. 3, 32

McKenna et al.(1999) S. McKenna, Y. Raja e S. Gong. Tracking colour objects using adaptive mixture models. Image and Vision Computing, 17(3-4):225-231. Citado na pág. 3

Mikolajczyk e Schmid(2005) K. Mikolajczyk e C. Schmid. A performance evaluation of local descriptors. IEEE Transactions on Pattern Analysis and Machine Intelligence, 27(10):1615-1630. Citado na pág. xiii, 9,14

Mitchell(1997) T. M. Mitchell. Machine Learning. McGraw-Hill Series in Computer Science. WCB/McGraw-Hill. Citado na pág. 87

Mottaghi(2012) R. Mottaghi. Augmenting deformable part models with irregular-shaped object patches. Computer Vision and Pattern Recognition, IEEE Computer Society Conference on. Citado na pág. 43

Nevatia e Binford(1977) R. Nevatia e T. O. Binford. Description and recognition of curved objects. Artif. Intell., 8(1):77-98. Citado na pág. 13

Niknejad et al.(2011) H. T. Niknejad, S. Mita, D. McAllester e T. Naito. Vision-based vehicle detection for nighttime with discriminately trained mixture of weighted deformable part models. Em Intelligent Transportation Systems (ITSC), 2011 14th International IEEE Conference on, páginas 1560-1565. IEEE. ISBN 978-1-4577-2198-4. doi: 10.1109/ITSC.2011.6082826. URL http://dx.doi.org/10.1109/ITSC.2011.6082826. Citado na pág. 14, 15

Niknejad et al.(2012a) Hossein Tehrani Niknejad, Taiki Kawano, Mikio Shimizu e Seiichi Mita. Vehicle detection using discriminatively trained part templates with variable size. Em Intelligent Vehicles Symposium, páginas 766-771. IEEE. ISBN 978-1-4673-2119-8. Citado na pág. 15

Niknejad et al.(2012b) Hossein Tehrani Niknejad, Akihiro Takeuchi, Seiichi Mita e David A. McAllester. On-road multivehicle tracking using deformable object model and particle filter with improved likelihood estimation. IEEE Transactions on Intelligent Transportation Systems, 13(2): 748-758. Citado na pág. 14, 15

Papageorgiou(2000) Constantine Phaedon Papageorgiou. A trainable system for object detection in images and video sequences. Tese de Doutorado. AAI0801978. Citado na pág. 14

Pepik et al.(2012) B. Pepik, M. Stark, P. Gehler e B. Schiele. Teaching 3d geometry to deformable part models. Em IEEE Conference on Computer Vision and Pattern Recognition (CVPR) 2012, Providence, RI, USA. accepted as oral. Citado na pág. 3

Platt(1999) J. Platt. Fast training of support vector machines using sequential minimal optimization. Advances in Kernel Methods - Support Vector Learning, páginas 185-208. Citado na pág. 13 
Rao(1997) Rajesh P. N. Rao. Dynamic appearance-based recognition. Em Proceedings of the IEEE Computer Society Conference on Computer Vision and Pattern Recognition, páginas 540546. Citado na pág. 2

Schapire(2002) Robert E. Schapire. The boosting approach to machine learning: An overview, 2002. Citado na pág. 13,14

Schiele(2012) Bernt Schiele. Teaching 3d geometry to deformable part models. Em Proceedings of the 2012 IEEE Conference on Computer Vision and Pattern Recognition (CVPR), CVPR '12, páginas 3362-3369, Washington, DC, USA. IEEE Computer Society. ISBN 978-1-4673-1226-4. URL http://dl.acm.org/citation.cfm?id=2354409.2354979. Citado na pág. 14, 15

Schneiderman e Kanade(2000) H. Schneiderman e T. Kanade. Statistical method for 3d object detection applied to faces and cars. Em Proceedings of the IEEE Computer Society Conference on Computer Vision and Pattern Recognition, volume 1, páginas 746-751. Citado na pág. 14, 15

Schölkopf et al.(2000) B. Schölkopf, A. J. Smola, R. C. Williamson e P. L. Bartlett. New support vector algorithms. Neural computation, 12(5):1207-1245. Citado na pág. 13

Schölkopf et al.(2001) B. Schölkopf, J. C. Platt, J. Shawe-Taylor, A. J. Smola e R. C. Williamson. Estimating the support of a high-dimensional distribution. Neural computation, 13(7):1443-1471. Citado na pág. 13

Sivic e Zisserman(2008) J. Sivic e A. Zisserman. Efficient visual search for objects in videos. Proceedings of The IEEE, 96:548-566. Citado na pág. xiii, 2, 3, 9, 15

Sivic et al.(2005) J. Sivic, B. C. Russell, A. A. Efros, A. Zisserman e W. T. Freeman. Discovering object categories in image collections. Em Proceedings of the International Conference on Computer Vision. Citado na pág. 9

Song et al.(2011) Z. Song, Q. Chen, Z. Huang, Y. Hua e S. Yan. Contextualizing object detection and classification. Computer Vision and Pattern Recognition, IEEE Computer Society Conference on, páginas 1585-1592. Citado na pág. 43

Sudowe e Leibe(2011) P. Sudowe e B. Leibe. Efficient use of geometric constraints for slidingwindow object detection in video, volume 6962 LNCS of Lecture Notes in Computer Science (including subseries Lecture Notes in Artificial Intelligence and Lecture Notes in Bioinformatics). Citado na pág. 3

Sun et al.(2006) Z. Sun, G. Bebis e R. Miller. On-road vehicle detection: A review. IEEE Transactions on Pattern Analysis and Machine Intelligence, 28(5):694-711. Citado na pág. 14

Szeliski(2010) Richard Szeliski. Computer Vision: Algorithms and Applications. Springer, 1st edition. edição. ISBN 1848829345. Citado na pág. 1

Theodoridis e Koutroumbas(2003) S. Theodoridis e K. Koutroumbas. Pattern recognition. Academic Press. Academic Press. Citado na pág. 88

Thomas et al.(2006) A. Thomas, V. Ferrari, B. Leibe, T. Tuytelaars, B. Schiele e L. V. Gool. Towards multi-view object class detection. CVPR. Citado na pág. 15

Torralba e Efros(2011) A. Torralba e A. A. Efros. Unbiased look at dataset bias. Em Proceedings of the 2011 IEEE Conference on Computer Vision and Pattern Recognition, CVPR '11, páginas 1521-1528, Washington, DC, USA. IEEE Computer Society. ISBN 978-1-4577-0394-2. doi: 10. 1109/CVPR.2011.5995347. URL http://dx.doi.org/10.1109/CVPR.2011.5995347. Citado na pág. xiv, 4, 29, 30, 84 
Torralba et al.(2008) A. Torralba, K. P. Murphy e W. T. Freeman. Sharing visual features for multiclass and multiview object detection. IEEE Trans.on PAMI. Citado na pág. 14, 15

Tsochantaridis et al.(2004) I. Tsochantaridis, T. Hofmann, T. Joachims e Y. Altun. Support vector machine learning for interdependent and structured output spaces. Em Proceedings, TwentyFirst International Conference on Machine Learning, ICML 2004, páginas 823-830. Citado na pág. 13

Vapnik(1998) V. N. Vapnik. Statistical Learning Theory. Citado na pág. 13

Vapnik(1995) V. N. Vapnik. The nature of statistical learning theory. Springer-Verlag New York, Inc., New York, NY, USA. Citado na pág. 13, 87

Vedaldi et al.(2009) A. Vedaldi, V. Gulshan, M. Varma e A. Zisserman. Multiple kernels for object detection. Em Proceedings of the International Conference on Computer Vision. Citado na pág. 43

Viola e Jones(2001) P. Viola e M. Jones. Rapid object detection using a boosted cascade of simple features. Em Proceedings of the IEEE Computer Society Conference on Computer Vision and Pattern Recognition, volume 1, páginas I511-I518. Citado na pág. xiii, 10, 14

Wang e Forsyth(2009) G. Wang e D. Forsyth. Joint learning of visual attributes, object classes and visual saliency. Em Proceedings of the IEEE International Conference on Computer Vision, páginas 537-544. Citado na pág. 16

Wang et al.(2008) G. Wang, D. Xiao e J. Gu. Review on vehicle detection based on video for traffic surveillance. Em Automation and Logistics, 2008. ICAL 2008. IEEE International Conference on, páginas 2961 -2966. doi: 10.1109/ICAL.2008.4636684. Citado na pág. 1, 14

Wang e Mori(2010) Y. Wang e G. Mori. A discriminative latent model of object classes and attributes, volume 6315 LNCS of Lecture Notes in Computer Science (including subseries Lecture Notes in Artificial Intelligence and Lecture Notes in Bioinformatics). Citado na pág. 16

Wren et al.(1997) C. Wren, A. Azarbayejani, T. Darrell e A. Pentland. Pfinder: Real-time tracking of the human body. IEEE Transactions on Pattern Analysis and Machine Intelligence, 19(7):780785. Citado na pág. 3

Yu e Joachims(2009) Chun-Nam John Yu e Thorsten Joachims. Learning structural svms with latent variables. Em Proceedings of the 26th Annual International Conference on Machine Learning, ICML '09, páginas 1169-1176, New York, NY, USA. ACM. ISBN 978-1-60558-516-1. doi: 10.1145/1553374.1553523. URL http://doi.acm.org/10.1145/1553374.1553523. Citado na pág. 13

Yuen et al.(2009) Jenny Yuen, Bryan C. Russell, Ce Liu e Antonio Torralba. Labelme video: Building a video database with human annotations. Em IEEE 12th International Conference on Computer Vision, ICCV 2009, Kyoto, Japan, September 27 - October 4, 2009, páginas 1451-1458. IEEE. doi: http://dx.doi.org/10.1109/ICCV.2009.5459289. Citado na pág. 34

Yuille et al.(1992) A. L. Yuille, P. W. Hallinan e D. S. Cohen. Feature extraction from faces using deformable templates. International Journal of Computer Vision, 8(2):99-111. Citado na pág. 8, 11,15

Zheng e Liang(2009) W. Zheng e L. Liang. Fast car detection using image strip features. Em 2009 IEEE Computer Society Conference on Computer Vision and Pattern Recognition Workshops, CVPR Workshops 2009, páginas 2703-2710. Citado na pág. 3, 15

Zhu et al.(2008) L. Zhu, C. Lin, H. Huang, Y. Chen e A. Yuille. Unsupervised structure learning: Hierarchical recursive composition, suspicious coincidence and competitive exclusion. ECCV. Citado na pág. 15 
Zhu et al.(2010) L. Zhu, Y. Chen, A. Torralba, W. Freeman e A. Yuille. Part and appearance sharing: Recursive compositional models for multi-view multi-object detection. Em Proceedings of the IEEE Computer Society Conference on Computer Vision and Pattern Recognition, páginas 1919-1926. Citado na pág. 11, 15 